\title{
Material and Energy Balances for Methanol from Biomass Using Biomass Gasifiers
}

\author{
R.L. Bain
}

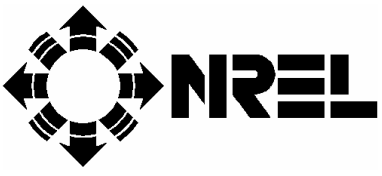

National Renewable Energy Laboratory 1617 Cole Boulevard Golden, Colorado 80401-3393

A national laboratory of the U.S. Department of Energy Managed by Midwest Research Institute for the U.S. Department of Energy under contract No. DE-AC36-83CH10093 


\section{Material and Energy Balances for Methanol from Biomass Using Biomass Gasifiers}

R.L. Bain

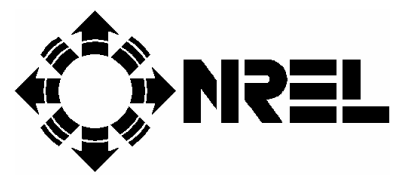

National Renewable Energy Laboratory 1617 Cole Boulevard

Golden, Colorado 80401-3393

A national laboratory of the U.S. Department of Energy Managed by Midwest Research Institute for the U.S. Department of Energy under contract No. DE-AC36-83CH10093

Prepared under Task No. BF15.3436

January 1992 


\section{NOTICE}

This report was prepared as an account of work sponsored by an agency of the United States government. Neither the United States government nor any agency thereof, nor any of their employees, makes any warranty, express or implied, or assumes any legal liability or responsibility for the accuracy, completeness, or usefulness of any information, apparatus, product, or process disclosed, or represents that its use would not infringe privately owned rights. Reference herein to any specific commercial product, process, or service by trade name, trademark, manufacturer, or otherwise does not necessarily constitute or imply its endorsement, recommendation, or favoring by the United States government or any agency thereof. The views and opinions of authors expressed herein do not necessarily state or reflect those of the United States government or any agency thereof.

Available to DOE and DOE contractors from:

Office of Scientific and Technical Information (OSTI)

P.O. Box 62

Oak Ridge, TN 37831

Prices available by calling 423-576-8401

Available to the public from:

National Technical Information Service (NTIS)

U.S. Department of Commerce

5285 Port Royal Road

Springfield, VA 22161

703-605-6000 or 800-553-6847

or

DOE Information Bridge

http://www.doe.gov/bridge/home.html 
MATERIAL AND ENERGY BALANCES FOR METHANOL FROM BIOMASS

USING BIOMASS GASIFIERS

\author{
Richard L. Bain \\ January 14, 1992
}

National Renewable Energy Laboratory

1617 Cole Boulevard

Golden, CO 80401-3393 


\section{MATERIAL AND ENERGY BALANCES FOR METHANOL FROM BIONASS USING BIOMASS GASIFIERS}

The objective of the Biomass to Methanol Systems Analysis Project (BF15.3436) is the determination of the most economically optimum combination of unit operations which will make the production of methanol from biomass competitive with or more economic than traditional processes with conventional fossil fuel feedstocks. One step in this process is the development of integrated methanol production simulation models. This report summarizes the development of simulation models for methanol production based upon the Institute of Gas Technology (IGT) "Renugas" gasifier and the Battelle Columbus Laboratory (BCL) gasifier. The IGT "Renugas" gasifier is a high-pressure, oxygen-blown, fluid-bed gasifier which has been operated at the 10 ton per day (TPD) pilot plant scale of operation on a number of biomass feeds and the $\mathrm{BCL}$ gasifier is a low pressure indirectly heated gasifier which has also been operated at the 10-TPD scale. This report discusses methanol production technology, the IGT and BCL gasifiers, analysis of IGT and $B C L$ gasifier data for gasification of wood, methanol production material and energy balance simulations, and one case study based upon each of the gasifiers.

The IGT model was used to perform a simulation for the Hawai $i$ Natural Energy Institute (HNEI), assuming IGT's experiment 13-G as input data. The simulation indicated that approximately 100.5 million gallons of methanol per year can be produced from 2,000 d tons per day (dTPD) of bagasse with an IGT gasifier operating at $1526^{\circ} \mathrm{F}$ and 319 psia. The $\mathrm{BCL}$ model was used to simulate and $\mathrm{BCL}$ gasifier/methanol synthesis system. The gasifier simulation included operation at $1675{ }^{\circ} \mathrm{F}, 20 \mathrm{psia}$, and a quench step. The simulation indicated that approximately 110.5 million gallons per year of methanol could be produced.

An addendum to this report will be issued by the end of the first quarter of 1992, in which the results of IGT gasifier operation at $1800^{\circ} \mathrm{F}$, and BCL gasifier operation with hot gas conditioning are shown. 


\section{Table of Contents}

Page

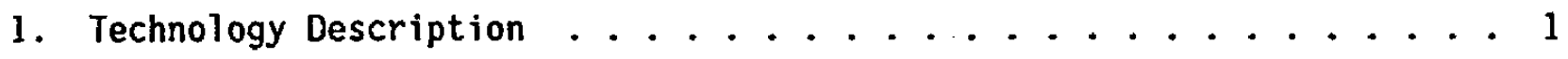

2. Analysis of IGT Biomass Gasification Data . . . . . . . . . 11

3. ASPEN Model for the IGT Gasifier System . . . . . . . . . . 12

4. IGT Case Study . . . . . . . . . . . . . . 16

5. Analysis of BCL Biomass Gasification Data .......... 23

6. ASPEN Model for the BCL Gasifier System . . . . . . . . . . 24

7. BCL Case Study ... . . . . . . . . . . . . 27

8. References ........................ 34

9. Appendices

1. IGT Gasifier Data . . . . . . . . . . . . . . 36

2. Least Squares Fit of Gasifier Variables .......... . 53

3. IGT Gasifier - Conditions and Correlations ........ . 58

4. ASPEN Input Data - IGT Gasifier . . . . . . . . . 60 60

5. ASPEN Program Listig - IGT Case . . . . . . . . . 66 63

6. Hawai Material Balance Information........... 74

7. BCL Gasifier Data . . . . . . . . . . . . . . . 82

8. BCL Least Squares Fits ............... . . . 98

9. BCL: Feed Analysis and Yield Correlations ........ 103

10. ASPEN Input file: BCL Recycle Case .......... 105

11. ASPEN Program Listing - BCL Case .......... . 116 


\section{List of Figures}

Page

1. Thermochemical Production of Methanol from Biomass ........ 3

2. Process Simulation Schematic - IGT Gasifier/

Methanol Synthesis .................. 13

3. Process Simulation Schematic - BCL Gasifier/

Methanol Synthesis, Part A .............. 25

4. Process Simulation Schematic - BCL Gasifier/

Methanol Synthesis, Part B .............. 26 


\section{Technology Description}

The thermochemical production of methanol from biomass involves the production of a synthesis gas rich in hydrogen and carbon monoxide which is then catalytically converted into methanol. Production of the synthesis gas is accomplished by thermal gasification.

The unit operations involved in methanol production from biomass are divided into the following major areas: 1) feed preparation, 2) gasification, 3) synthes is gas modification, and 4) methanol synthesis and purification. In order to understand the need for these processing steps a brief discussion of the chemistry of methanol production is required. Wood will be used as a typical biomass feedstock. Wood is a complex mixture (Graboski and Bain 1979) of organic compounds and polymers. The major types of compounds are lignin and carbohydrates (cellulose and hemicellulose) whose ratios and resulting properties are species dependent. Lignin, the cementing agent for cellulose is a complex polymer of phenylpropane units. Cellulose is a polymer formed from $d(t)$-glucose while the hemicellulose polymer is based on hexose and pentose sugars. Wood has low ash, nitrogen, and sulfur contents. In order to estimate yields during gasification the complex material must be reduced to a simplified chemical formula, such as $\mathrm{CH}_{1.4} \mathrm{O}_{0.6}$. Elements such as sulfur and nitrogen are typicaliy present in very small amounts and do not need to be considered in terms of overall chemistry.

The combustion of wood can be ideally represented by:

$$
\mathrm{CH}_{1.4} \mathrm{O}_{0.6}+1.05 \mathrm{O}_{2} \cdots+\mathrm{CO}_{2}+0.7 \mathrm{H}_{2} \mathrm{O}
$$

Oxygen blown gasification can be thought of as incomplete combustion or partial oxidation. Gasification using a minimum amount of oxygen can be represented by:

$$
\mathrm{CH}_{1.4} \mathrm{O}_{0.6}+0.2 \mathrm{O}_{2} \quad \cdots \quad \mathrm{CO}+0.7 \mathrm{H}_{2}
$$

In cases where no oxygen is used an "ideal" gasification reaction can be represented by: 


$$
\mathrm{CH}_{1.4} \mathrm{O}_{0.6} \quad \cdots \quad 0.6 \mathrm{CO}+0.4 \mathrm{C}+0.7 \mathrm{H}_{2}
$$

This pyrolysis reaction is endothermic and heat is needed to make the reaction proceed. This heat is provided by the oxidation reactions shown above or by indirect heat transfer. While these ideal reactions are simple, actual gasification is more complex and intermediate compounds such as tars and methane are formed which must be further processed before the synthesis gas can be used to produce methanol.

Methanol is formed catalytically by the following reaction:

$$
\mathrm{CO}+2 \mathrm{H}_{2} \quad \longrightarrow-->\mathrm{CH}_{3} \mathrm{OH}
$$

It can be seen that two molecules of hydrogen are required for each molecule of carbon monoxide. Gasification may produce a gas with a hydrogen to carbon monoxide ratio as low as one-half. In this case water is added and some of the carbon monoxide is used to produce hydrogen by the catalytic shift conversion reaction:

$$
\mathrm{CO}+\mathrm{H}_{2} \mathrm{O} \quad-\cdots>\mathrm{CO}_{2}+\mathrm{H}_{2}
$$

The methanol synthesis reaction is an equilibrium reaction and does not proceed to completion. In order to obtain economic yields unreacted gas is recycled to the synthesis reactor. While not detrimental to process chemistry, inert gases such as methane must be purged from the system, resulting in loss of yield and an economic penalty. Concentrations of methane larger than one or two percent typically result in unacceptable economic penalties. Therefore, synthesis gases containing high levels of methane are steam reformed prior to methanol synthesis. The primary catalytic reforming reaction is:

$$
\mathrm{CH}_{4}+\mathrm{H}_{2} \mathrm{O} \quad-\cdots>\mathrm{CO}+3 \mathrm{H}_{2}
$$

In addition the shift conversion reaction shown above also occurs in the reformer. 
Carbon dioxide also reacts with hydrogen to produce methanol, but consumes more hydrogen per mole of methanol formed than when using carbon monoxide. Most of the carbon dioxide is therefore removed from the synthesis gas prior to methanol synthesis.

With this knowledge of chemistry as a basis the unit operations can be discussed. Figure 1 shows the major routes for production of methanol from biomass on a simplified basis. These routes differ primarily in the type of gasification process chosen.

\section{Thermochemical Routes to Methanol from Biomass}

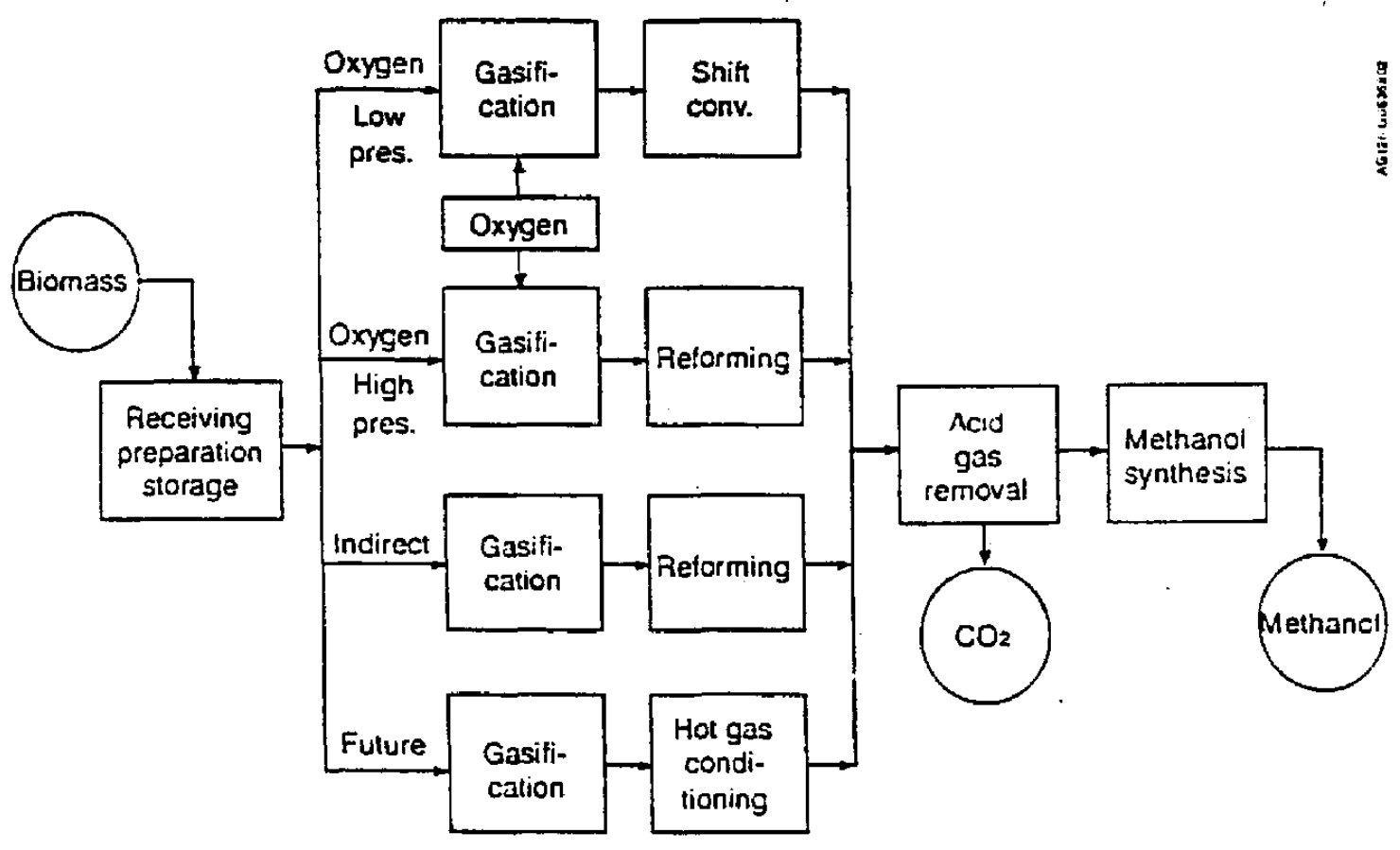


The feed preparation section of a biomass to methanol process involves wood storage and handling, size reduction, and drying. Size reduction is process specific. Drying is performed to minimize feed degradation during storage and to optimize the overall process energy balance. Orying to $10 \%$ to $15 \%$ moisture content is accomplished using waste process heat. This waste heat may come from various unit operations in an integrated methanol production facility. For a system with an indirect gasifier, this waste heat comes from hot flue gases produced during char combustion. For a direct oxidative gasifier system the waste heat comes from reformer furnace flue gases. The heat required to dry biomass from $50 \%$ moisture to $10 \%$ moisture represents about $10 \%$ of the heating value of the biomass.

Gasifiers can be divided into three major classes: 1) air gasifiers, 2) oxygen gasifiers, and 3) indirect gasifiers. This classification is based primarily upon the method of supplying the heat necessary to drive the endothermic pyrolysis reactions, the carbon-steam reaction and the carbon-carbon dioxide reaction. Gasification is an old technology for converting coal and biomass into a gas which can be used in various technical processes. Coal gasification is commercial technology used to produce substitute natural gas (Great Plains), gasoline and diesel fuel (Sasol), and methanol (Tennessee Eastman as an intermediate in acetic anhydride production and $\mathrm{SASOL}$ as an intermediate in formaldehyde production). Biomass gasification was used in Europe during Worid War II to suppiy energy for transportation, electricity and heat.

Biomass gasification has not been commercially developed in this country because of the abundant supplies of natural gas, petroleum, and coal. Because of the differences between coal and biomass, coal gasifiers are not directly usable for biomass gasification. Differences in reactivity (with biomass being more reactive) change required operating temperatures, pressures, and residence times. Difference in density between coal and biomass requires modification of the solids feeding systems. The ancillary facilities, such as utilities and waste treatment can be applied to biomass gasification except that biomass gasifiers do not require as extensive clean up for sulfur or nitrogen derived compound emissions as do coal gasifiers because of the low sulfur and nitrogen content of wood. A number of gasifiers are being developed in this country, in Canada, in 
Europe, and in other countries to process biomass. Because of the high reactivity of biomass they are typically operated at lower temperatures than are coal gasifiers. To date, gasifier development has concentrated on production of low and medium BTU gas for use in electrical generation and as a substitute natural gas. Development has not been specific for methanol production.

Air gasifiers use the oxygen in air to provide process heat. A portion of the feed is burned, and the heat of combustion is used to gasify the remaining feed. The nitrogen present in air acts as a diluent in methanol production and leads to unattractive economics. Air gasification product can be used for electricity generation, and for ammonia synthesis.

In order to reduce the amount of inert gas in the gasifier product stream relatively pure oxygen can be used in place of air. While the use of oxygen will produce a gas suitable for downstream synthesis gas processing, oxygen is expensive and accounts for a large percentage of plant capital and operating costs. For example, oxygen costs $\$ 40$ to $\$ 60$ dollars per ton, and is typically used at the rate of 0.25 to 0.35 ton oxygen per ton of biomass in oxygen gasification. This transiates to a cost of $\$ 10$ to $\$ 21$ per ton of biomass processed.

Oxygen gasifiers are operated at both low and high pressure. A low pressure oxygen (LPO) gasifer presently being evaluated for biomass gasification is the Koppers-Totzek (K-T) gasifier. The K-T gasifier (Probstein and Hicks 1982, Chem Systems 1990 ) is an entrained flow gasifier whose operation requires that the biomass be ground very fine, minus 30 mesh (minus ca. 0.02 inches). The required comminution adds appreciably to feed preparation costs. Operation at low pressure in the presence of oxygen produces little methane and tars. The hydrogen to carbon monoxide ratio is less than one, comparable that of indirect gasifiers. The Union Carbide Corporation Purox (Keenan 1977) process has been developed for municipal solid waste gasification. The hydrogen to carbon monoxide ratio in the produced gas is also less than one.

High pressure oxygen (HPO) gasifiers are being developed to improve on the economics of LPO gasifiers. Typically these gasifiers are fluid bed gasifiers 
which are fed fairly large wood chips, e.g. minus 2 inches. 0xygen and steam are injected near or at the bottom of the reactor and react with the wood, char and synthesis gas. Fluid bed reactors have the advantage of good mixing of the feed solids, uniform bed temperature, and rapid equilibrium between solids and gases. However, operation at high pressure favors the formation of methane. Operation at high pressure reduces gasifier capital cost and downstream compression costs, but downstream processing to remove or reform tars and methane adds appreciably to capital and operating costs. The Winkler and Institute of Gas Technology (IGT) gasifiers are representative of HPO fluid bed gasifiers. The Texaco gasifier is a representative HPO entrained flow gasifier. The Winkler and Texaco gasifiers have been developed for coal. The IGT gasifier (Evans et al 1988) is designed for biomass operation and has been operated at the twelve ton per day scale.

Indirect (IND) gasifiers produce a solid carbon-rich char, see equation 3 , which is reacted with air in a separate combustor to provide process heat. This heat is transferred to the gasifier by circulation of hot inert solids, or by indirect heat transfer through the walls of the gasifier or through the walls of heat exchange tubes. IND gasifiers typically produce a synthesis gas rich in carbon monoxide, and with low carbon dioxide levels. In order to produce sufficient char to provide all the heat necessary for gasification these gasifiers are normally operated at relatively low temperatures, 1300 to $1600^{\circ} \mathrm{F}$. At these temperatures synthesis gas yields are reduced and methane concentration is high. The addition of a catalyst may improve the hydrogen to carbon monoxide ratio substantially. Downstream reforming is required for methanol synthesis. Operation of developmental reactors has been directed to production of medium BTU gas, not toward methanol synthesis gas production. The Battelle-Columbus Laboratory (BCL) gasifier (Feldmann et al, 1988) and the University of MissouriRolla (UMR) gasifier (Flanigan et al 1988) are typical of IND gasifiers developed for biomass processing. Both of these gasifiers have been operated at the pilot scale.

The synthesis gas exiting the gasifier contains small amounts of tar and char which must be removed prior to downstream catalytic conversion operations. Typically, gasification systems use scrubbers to remove tars. While efficient in 
contaminant removal scrubbers produce a dirty water stream which must be further processed. An alternative to scrubbing is hot gas cleanup. In coal gasification systems operated at high temperature tar removal is generally not required, and hot gas cleanup is directed toward removal of sulfur compounds. Hot gas cleanup systems are being developed for biomass gasifiers in Europe. The Studsvik MINO process (Rensfelt and Ekstrom 1989) in Sweden includes a catalytic tar conversion operation and has been operated at a pilot scale. In France the Cruesot Loire system (Philip 1986) uses a thermal tar conversion reactor. Research is ongoing in the United States in the area of hot gas cleanup for biomass gasifiers, but large pilot operations have not been undertaken to date.

All unit operations downstream of the gas cleanup operation are commercial technology, although potentially improved technologies are being investigated. Steam-methane and steam-naphtha reformers are the primary method of production of hydrogen by the petroleum industry and have been operated for many years. Likewise, shift conversion reactors have been operated commercially for many years as a part of steam-reformer systems. In 1989 approximately 7,345 million gallons of methanol production capacity existed worldwide (Crocco 1989) using thermal conversion operations. Eighty-six percent of this production capacity uses steam-reforming operations followed by catalytic methanol synthesis. The primary commercial methanol synthesis processes are licensed by ICI and Lurgi.

A liquid phase methanol synthesis concept is being investigated by Brookhaven National Laboratory (Mahajan etal 1989) in which a novel liquid phase catalyst is used to catalytically convert synthesis gas to methanol at low temperature (ca. 110-130 ${ }^{\circ} \mathrm{C}$ ) and low pressure (ca. 180-360 psia). Single pass conversions of 90 percent of the 1 imiting reactant, typically $\mathrm{CO}$, with high selectivity, over 95 percent, to methanol have been reported. Another liquid phase methanol process is under development by Air Products and Chem Systems (Studer eta1 1989) in which a solid catalysts is suspended in an organic solvent. The process has been tested at the PDU scale, up to 12 TPD of methanol production, for extended periods of time on $\mathrm{CO}$ rich gases. Thermal efficiencies are reported to be 90-94 $\%$ for the synthesis step. These single pass methanol synthesis processes will most probabiy be best utilized in conjunction with syngas generation processes producing low methane content syngas, or in a combined process where the 
unreacted methanol synthesis gas is used for electricity generation.

Technical development efforts for production of methanol from biomass are concentrated in the area of gasification. As stated before these efforts are primarily directed toward production of medium BTU gas for electrical generation or for fuel use and not for synthesis gas production. However, results obtained are directly applicable to gasification for methanoi production. Gasification systems are being developed in the United States, in Canada, and in Europe. This discussion will concentrate on biomass gasifiers being developed in the United States.

Five gasifier systems are actively being developed in the United States for biomass gasification. These systems are listed below:

- Battelle Columbus Laboratories (BCL)

- Institute of Gas Technology (IGT)

- Manufacturing and Technology Conversion International Incorporated (MTCI)

- Syngas, Inc. (SGI)

- University of Missouri-Rolla (UMR)

BCL Gasifier

The BCL gasifier system (Feldmann et.al., 1988) is a dual bed IND gasifier system operated in an entrained flow mode. Heat for gasification is supplied by hot sand recirculating between a separate combustion vessel and the gasifier. Residual char remaining after gasification of the wood provides the fuel for the gasifier. The system has been operated at a 25 ton per day scale in pilot plant operation. The gasifier produces a synthesis gas with a low hydrogen to carbon ratio, high methane content and some tars. For methanol production the tars will have to be removed and the methane reformed to produce a suitable synthesis gas. The pilot system has been operated under conditions giving energy self sufficiency on a gasifier stand alone basis. It is possible that higher temperature operations are possible in an integrated plant where a portion of purge gases from the methanol synthesis loop can be used to make up shortfalls 
in heat available from char combustion. At higher temperatures tar yield and methane content will both be reduced.

\section{IGT Gasifier}

The IGT gasifier (Evans et.al., 1988) is a HPO gasifier operated in the fluid bed mode. Oxygen and steam are introduced near the bottom of the fluid bed reactor. oxygen reacts with a portion of the feed and gasification products to supply the heat required for gasification. The system is designed to produce a medium BTU gas from biomass at moderate temperatures, $1400^{\circ}$ to $1800^{\circ} \mathrm{F}$ and high pressures, 100 to $350 \mathrm{psia.}$. The IGT gasifier has been operated at a 12 tons per day scale. The product gas is high in methane and contains some tars. For methanol production tars will have to be removed and the gas reformed to reduce the methane concentration.

\section{MTCI Gasifier}

The MTCI gasifier (MTCI 1990) is an IND gasifier operated in the fluid bed mode at moderate temperatures, $\mathrm{Ca} .1200^{\circ}$ to $1300^{\circ} \mathrm{F}$ and atmospheric pressure. Heat for the gasification reaction is supplied indirectly through heat exchange tubes placed in the fluid bed. A pulse combustion system is used to increase the rate of heat transfer from the combustion flue gas to the fluid bed. To date natural gas has been used as the fuel for combustion, but a portion of the produced gas would probably be used for commercial operations. In addition to the high heat transfer rate, operation of the system is characterized by the use of a catalytic fluidization solid which results in product gases having high hydrogen to carbon monoxide ratios. As for the previous gasifiers the product gas contains methane and tars and will require cleanup and reforming in commercial operations. Only limited pilot runs have been performed at a 0.4 ton per day scale. For methanol production tars will have to removed, and the gas reformed to produce a suitable synthesis gas, assuming operation at conditions comparable pilot operation conditions.

SGI Gasifier 
The SGI gasifier (Reed et. al., 1988) is a stratified downdraft gasifier which can be operated using air or oxygen as a LPO or HPO gasifier. The system has been operated on a limited basis as a HPO gasifier at the 24 ton per day scale. Original development of the gasifier was performed by the Solar Energy Research Institute from 1981 to 1985 . The technology was licensed to SynGas, Inc., in the mid 80's for commercial development. The unit operates as a moving bed gasifier with co-current flow of oxygen or air in a downward direction. The design produces a minimum of tars. The system is designed to produce a low to medium BTU fuel gas containing a low hydrogen to $\mathrm{CO}$ ratio, some methane, and some tars.

\section{UMR Gasifier}

The UMR gasifier (Flanigan et.al., 1988) is an IND gasifier operated as a fluid bed reactor with heat supplied via heat exchanger tubes internal to the bed. Heat is supplied by high temperature combustion flue gas. In pilot operations natural gas has been used for combustion fuel, but in commercial operation char or a portion of gasifier product gas would be used. The system has been operated at the 3.6 short ton per day scale at relatively low temperatures. Operation at low temperatures gives higher char and tar yields and lower gas yields than the other gasifiers under development. Temperature may be limited by maximum indirect heat transfer rates. The gas will have to be reformed and tar destruction will be required to make a suitable synthesis gas feed. Because of operation at low temperature the tar production in this gasifier is an order of magnitude larger than in the other gasifiers. Higher temperature or cata 7 bed operation of the system would produce a product stream similar to that of = MTCI gasifier.

To summarize technology status, a number of gasifiers are under development which have the potential to produce a synthesis gas suitable for methanol synthesis. These gasifiers are operating in the 4 to 25 ton per day scale. All systems under development are designed to produce a low to medium BTU fuel gas. None of the systems have been operated on an integrated process basis to determine operating parameters necessary for maximum methanol production. All downstream synthesis gas operations are commercial technology in which operating conditions and yields are known. 


\section{Analysis of IGT Biomass Gasification Data}

The objective of modeling the IGT gasification experimental data was to put the gasifier yield data in a form which could be used as input information in the ASPEN material and energy balance simulation. The experimental data were taken from the 1988 IGT report to Pacific Northwest Laboratories (Evans et.al., 1988). The following procedure was used convert the experimental data:

1. The experimental gasifier results given by Evans were input into a Lotus Spreadsheet. The data are given in Appendix 1.

2. The experimental yield was modified to force a $100 \%$ material balance. The modified data are given in Appendix 1 .

3. Yields and gas compositions were correlated using linear least squares fits as polynomial functions of temperature. The least squares results are given in Appendix 2. A summary of gasifier conditions, feed properties, and yield correlations is given in Appendix 3.

4. The yield correlation were then used to generate ASPEN input data. The gasifier reactor model used to represent the gasifier in the ASPEN simulation is a $R$-YIELD reactor in which a chemical reactor is simulated by specifying component yields. This type of reactor is used when reaction stoichiometry and kinetics are unknown but yields distribution data or correlations are available. The ASPEN input data are shown in Appendix 4. 
The IGT gasifier based system simulated for this study is given by Figure 2 . The major process components simulated were:

1. Feed dryer

2. Gasifier with no quench

3. Preformer: This reactor operates much like a naphtha preformer where higher hydrocarbons are converted to methane. The preformer was simulated as a stoichionetric reactor

4. Reformer: In the reformer methane reacts with steam to produce hydrogen and carbon monoxide. This reactor was modelled as a RGIBBS reactor, assuming equilibrium based upon minimization of Gibbs Free Energy. The reactions included in the simulation were the steam reforming reaction, the water gas shift reaction, and the $c 0$ decomposition reaction to form carbon and carbon dioxide. A steam reforming lack of equilibrium was assumed by using a minus $15^{\circ} \mathrm{F}$ approach temperature for the steam reforming reaction.

5. Acid gas removal and recycle: This module is not rigorously modelled in this present simulation. The product stream is cooled and separation is forced by the simulation program. This module should be changed in the future to simulate a Benfield or Catacarb unit. A portion of the recovered carbon dioxide is recycled to the preformer.

6. The methanol compressor is modelled as a centrifugal compressor.

7. Methanol synthesis: The methanol synthesis is simulated as an equilibrium reactor. The reactions used are the methanol reaction and the water gas shift reaction. 


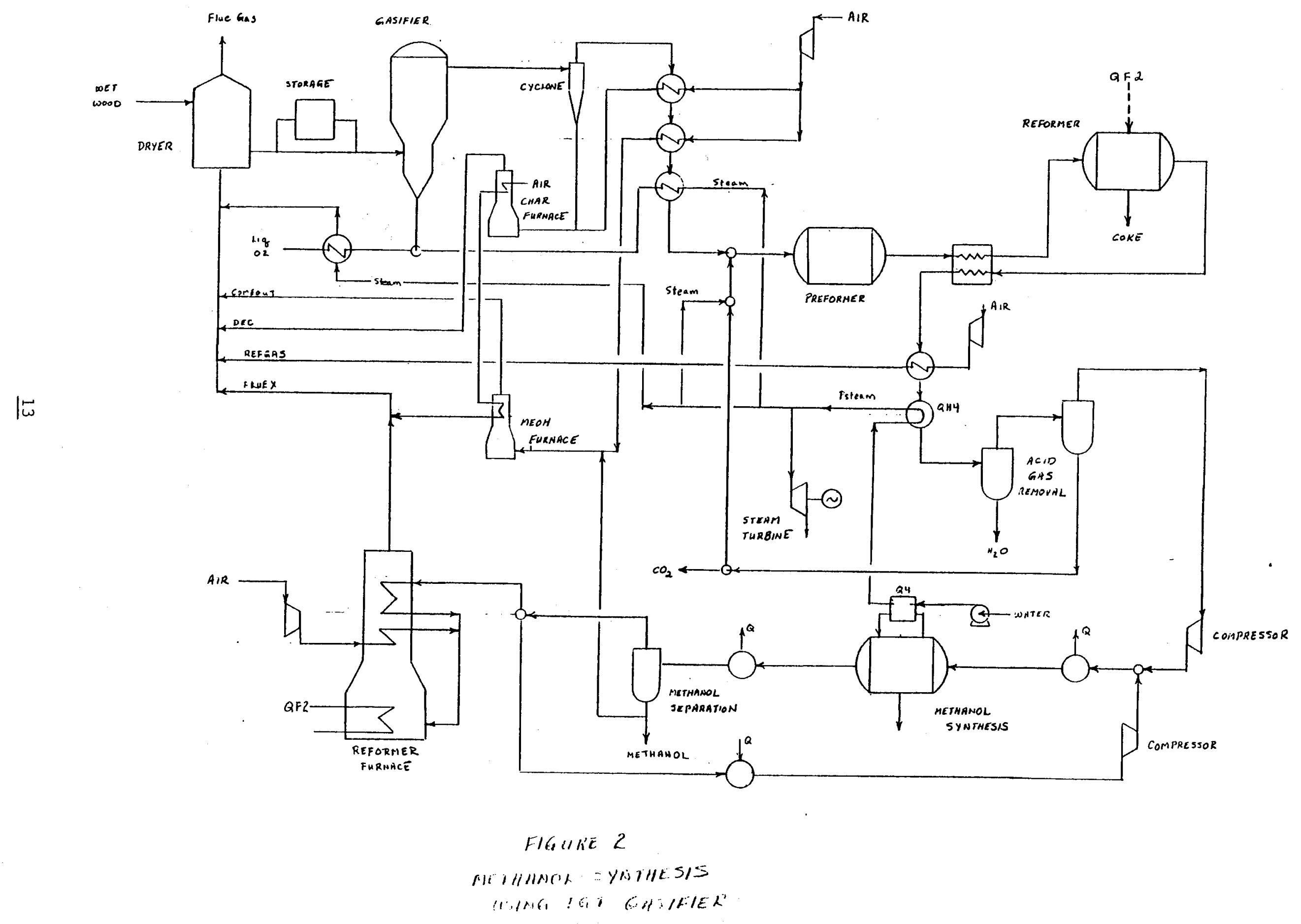


8. Methanol recovery is simulated by cooling the methanol reactor product stream, and performing a flash calculation. The raw methanol stream is removed. Methanol purification is not simulated. A portion of the remaining gas is recycled to the methanol reactor, and the rest is sent to the reformer furnace. 
9. Reformer furnace: Methanol synthesis purge gas is burned using a stoichiometric reactor. The assumed approach temperature between furnace flue gas and reformer exit temperature is $100{ }^{\circ} \mathrm{F}$. The flue gas from the reformer furnace is used to raise process steam, then fed to the dryer.

10. Steam Generation: Water is heated using the heat given off in the methanol synthesis reaction, and in cooling excess water from the acid gas removal operation. The amount of excess steam is dependent upon heat recovery in the acid gas plant. Future changes in the simulation will improve the accuracy of this value, and affect the amount of electricity required.

11. Electricity Generation: Excess steam is fed to a steam turbine for production of process electricity.

A listing of the simulation program is given in Appendix 5: 


\section{IGT Case Study}

The IGT simulation model was used to perform a simulation for the Hawaii Natural Energy Institute (HNEI). For this case study HNEI requested that IGT's experiment $13-G$ be used as input data. Therefore, the data from this experiment were normalized to ASPEN input form. The normalization procedure is given in Appendix 6 . The preliminary analysis is given below. In summary, the simulation indicates that approximately 100.5 million gallons of methanol per year can be produced from bagasse using a 2000 dTPD IGT gasifier operating at $1526^{\circ} \mathrm{F}$, and 319 psia. This production rate compares to 101.5 million gallons per year predicted by Chem Systems (Chem Systems, 1990) for the same gasifier operating at $1800^{\circ} \mathrm{F}$ and 500 psia.

Basis: 2000 dTPD Feed

Feed Analysis defined per Hawai i System A data

I. System Configuration:
a. Feed dryer
b. Gasifier with no quench
c. Preformer
d. Reformer
e. Acid gas removal
f. Compression
g. Methanol Synthesis
h. Purge Gas Combustion (Reformer heat input)

II. Feed:
Total
Carbon
$1.333 \mathrm{E}+09 \mathrm{1b} / \mathrm{yr}$ $0.6387 E+09 \mathrm{ib} / \mathrm{yr}$
$H H V=0.1439 E+10 \mathrm{Btu} / \mathrm{hr}$

III. Product Streams:

A. Methanol:

Overali

$2582.411 \mathrm{lbmol} / \mathrm{hr}=100.45 \mathrm{MM} \mathrm{gal} / \mathrm{yr}=0.66196 \mathrm{E}+09 \mathrm{lb} / \mathrm{yr}$ $49.65 \%$ of dry wood $\mathrm{HHV}=64,290 \mathrm{Btu} / \mathrm{lb}=0.8072 \mathrm{E}+09 \mathrm{Btu} / \mathrm{hr}=56.10 \%$ Carbon

$0.2481 E+09=38.84 \%$ of carbon in

B. Carbon Dioxide
Overall
Carbon
$0.89894 \mathrm{E}+09 \mathrm{lb} / \mathrm{yr}$
$0.24534 \mathrm{E}+09 \mathrm{ib} / \mathrm{yr}=38.41 \%$

IV. Carbon Balance Check

$$
\mathrm{ib} / \mathrm{yr}
$$


Methano1

$\mathrm{CO} 2$

Flue Gas

Assoc. MeOH

Total
$0.2481 E+09$
$0.8453 E+09$
$0.1234 E+09$
$0.0236 E+09$
38.8

38.4

19.3

3.7 (Assoc. CO, CO2, CH4)

\section{2}

V. UNIT OPERATIONS

A. Dryer

$T=220^{\circ} \mathrm{F}$

Qd $\quad=0.008876 \mathrm{E}+09 \mathrm{Btu} / \mathrm{hr}=0.62 \%$

Qwg $=0.03265 \mathrm{E}+09 \mathrm{Btu} / \mathrm{hr}=2.27 \%$

Qcond $=0.2295 \mathrm{E}+09 \quad$ Btu $/ \mathrm{hr}=15.95 \%$

B. Storage

Not used in this case

c. Gasifier

$\mathrm{T}=1526^{\circ} \mathrm{F}, \mathrm{P}=319.1 \mathrm{psia}$

$\mathrm{Qg}=0.0303 \mathrm{E}+09 \mathrm{Btu} / \mathrm{hr}=2.11 \%+2.7 \% \mathrm{IGT}$ correction $=4.81 \%$

D. Solid Separation

Ash $=0.0014 \mathrm{E}+09=0.10 \%$

E. Air Compressors

Air 1

Air 2

Airx

Refgas 1

Ambair
$3916 \mathrm{lbmol} / \mathrm{hr}$

50

6000

4000

5500

$833.11 \mathrm{hp}$

$19466 \mathrm{lbmol} / \mathrm{hr}$

Total air compressor horsepower:

$$
(19466 / 5500) *(833)=2948 h p \approx 3000 \mathrm{hp}
$$

Cost estimate

Reference: Garrett, D.E., Chemical Engineering Economics, using figure on page 271

Use 3 compressors, 1000 hp ea, 150 psia rating, Add 1 compressor as spare 
Purchase Price $=\$ 60,000(1987)$

Module factor $=2.6$

No. units $=4$

Installed Cost $(1987 \$)=60,000 * 2.6 * 4=\$ 624,000$

F. Reformer Loop

1. Inlet Conditions

$T=1261^{\circ} \mathrm{F}$

$P=319.1$ psia

Steam Added $=62001 \mathrm{bmole} / \mathrm{hr}$

2. Preformer - Converts non-methane hydrocarbons

$T=1196^{\circ} \mathrm{F}$

3. Preheater

Tout $=1404^{\circ} \mathrm{F}$

$Q=0.04773 \mathrm{~F}+09 \mathrm{Btu} / \mathrm{hr}$

$A=1595 \mathrm{ft}^{2}$

4. Reformer

$T=1600^{\circ} \mathrm{F}$

$P=319.1$ psia

$Q=-0.1744 \mathrm{E}+09 \mathrm{Btu} / \mathrm{hr}$

Gas Composition

$\begin{array}{lcc}\text { Comp } & \text { Mole \% } & \text { Mole \% Dry } \\ \text { H2 } & 26.38 & 47.39 \\ \mathrm{CO} & 12.29 & 22.08 \\ \mathrm{CO} 2 & 16.68 & 29.96 \\ \mathrm{H} 2 \mathrm{O} & 44.33 & -. \\ \mathrm{CH} 4 & 0.31 & 0.56\end{array}$

$\mathrm{H} 2 / \mathrm{CO} \quad 2.15$

5. $\mathrm{CO2}$ recycle

Product $/$ Recycle $=2553 / 1258=2.03$

G. Methanol Synthesis

1. Compressor 2

Pin $=319$ psia

Pout $=750$ psia 
$\operatorname{Tin}=300^{\circ} \mathrm{F}$

Tout $=514^{\circ} \mathrm{F}$

$\eta=0.95$

$H p=6248 \approx 6250$

Cost estimate:

Reference: Garrett, D.E., Chemical Engineering Economics,

from figure on page 272

Use 3 compressors $(2+1$ spare) rated at $3200 \mathrm{hp}$ ea

Base Cost $(1987 \$)=\$ 650,000$

Number $=3$

Module factor $=2.6$

Stainless steel $=2.5$

Pressure factor $=0.9495$

Cost $(\$ 1987)=3 * 650,000 * 2.6 * 2.5 * .9495$

$=12.04 \mathrm{MM} \mathrm{S}$

This cost compares to 7.6 MMs in Chem Systems report for Pin $=500$ psia.

2. Htr 5 (Recycle Preheat - to eliminate condensation in recycle compressor) $Q=-0.0038 E+09 \mathrm{Btu} / \mathrm{hr}$

3. Recycle Compressor; $30 \mathrm{HP}$

4. Htr 6 (Used to Balance compressor preheat)

$Q=.00456 E+09 \mathrm{Btu} / \mathrm{hr}$

$0.00456>0.00381 \quad 0 . K$.

5. Methanol Synthesis

$T=445^{\circ} \mathrm{F}, \mathrm{P}=750 \mathrm{psia}$

$Q=0.1144 \mathrm{E}+09 \mathrm{Btu} / \mathrm{hr}$

Note: Q + Agas Cooler Q used for steam production

6. Product Cooling $Q=0.0825 E+09 \mathrm{Btu} / \mathrm{hr}=5.73 \%$

Not used in present case

7. MeOH Separation

$Q=0.000408 E+09=0.03 \%$

Raw Product Liquid Composition

Comp 1bmol/hr Mole \% 


$\begin{array}{lrc}\mathrm{H} 2 & 3.11 & 0.10 \\ \mathrm{CO} & 1.33 & 0.005 \\ \mathrm{CO} 2 & 242.11 & 8.36 \\ \mathrm{H} 2 \mathrm{O} & 63.04 & 2.17 \\ \mathrm{CH} 4 & 2.71 & 0.009 \\ \mathrm{MeOH} & 2582.42 & 89.21\end{array}$

8. Recycle/Purge $=4360 / 2147=2.03$

H. Reformer Combustor (Furnace)

1. Air . Compressor - shown earlier in section II-E as ambair compressor.

2. Air Preheater

$$
\begin{aligned}
& Q=0.0443 \mathrm{E}+09 \mathrm{Btu} / \mathrm{hr} \\
& A=472 \mathrm{ft}^{2}
\end{aligned}
$$

3. Feed Gas Preheater

$$
\begin{aligned}
& Q=0.0111 E_{2}+09 \\
& A=169 \mathrm{ft}
\end{aligned}
$$

4. Combustor

$$
\begin{aligned}
& T=1743{ }^{\circ} \mathrm{F}>1700{ }^{\circ} \mathrm{F} \text { (Assumed } 100^{\circ} \mathrm{F} \text { approach in } \\
& \text { reformer furnace. }
\end{aligned}
$$

Excess Heat $=0.002667 \mathrm{E}+09 \mathrm{Btu} / \mathrm{hr}=0.18 \%$

1. Steam Balance

1. Generated $18,650 \mathrm{lbmol} / \mathrm{hr}$ at $319.1 \mathrm{psia}, 902.1^{\circ} \mathrm{F}$.

$$
\begin{aligned}
& \text { 2. Steam } \mathrm{G}-5365.9 \mathrm{lb}-\mathrm{mol} / \mathrm{hr} \\
& \text { Steam }-6200 \quad \mathrm{bb}-\mathrm{mol} / \mathrm{hr} \\
& \text { Steam02 }-1800 \mathrm{lb}-\mathrm{mol} / \mathrm{hr} \\
& \text { Steam2 }-5284 \mathrm{lb}-\mathrm{mol} / \mathrm{hr}
\end{aligned}
$$

ง. Steam Turbine

$$
\begin{aligned}
& \text { Inlet: } \quad T=900.9^{\circ} \mathrm{F} \\
& P=319.1 \text { psia } \\
& G=5284 \mathrm{lb}-\mathrm{mol} / \mathrm{hr} \\
& \text { Exit: } \quad T=213.1^{\circ} \mathrm{F} \\
& P=15.0 \text { psia } \\
& \text { Electricity }=0.02987 \mathrm{E}+09 \mathrm{Btu} / \mathrm{hr}=2.08 \% \\
& =8.75 \mathrm{MW} \\
& \text { Generator Loss }=0.0016 \mathrm{E}+09 \mathrm{Btu} / \mathrm{hr}=0.11 \% \\
& \text { Steam Condensation }=7.30 \%
\end{aligned}
$$

K. Preliminary Electrical 
Water Pump

Air Comp

MeOH Comp

Recycle Comp

Subtota 1

Dryer/Conveyer/Feeder

Acid Gas

Misc

L. Energy Balance

Methano]

Dryer

Wet Gas

Condense 1

Gasifier

Ash

Water

$\mathrm{MeOH} \mathrm{COO} 1$

W Turb

Gen. Loss

Comb Excess

Steam Cond
$118.4 \mathrm{Hp}$

3000

6250

30

$9398.4 \mathrm{Hp}=7 \mathrm{MW}$
56.10

0.62

2.27

15.95

4.81

0.10

4.06

5.73

2.08

0.11

0.05

7.30

99.3 
Gas Composition, Major Streams

\begin{tabular}{|c|c|c|c|c|c|c|c|}
\hline Comp \Stream & $\begin{array}{l}\text { Gasiffer } \\
\text { Product }\end{array}$ & $\begin{array}{c}\text { Preformer } \\
\text { Feed }\end{array}$ & $\begin{array}{l}\text { Preformer } \\
\text { Product }\end{array}$ & $\begin{array}{c}\text { Reformer } \\
\text { Product }\end{array}$ & $\begin{array}{l}\text { Methanol } \\
\text { Feed }\end{array}$ & $\begin{array}{l}\text { Methanol } \\
\text { Product }\end{array}$ & $\begin{array}{l}\text { Recycle } \\
\text { Gas }\end{array}$ \\
\hline $\begin{array}{l}\mathrm{H} 2 \\
\mathrm{CO} \\
\mathrm{CO} 2 \\
\mathrm{H} 20 \\
\mathrm{CH} 4 \\
\mathrm{C} 2 \mathrm{H4} 4 \\
\mathrm{C} 2 \mathrm{H6} \\
\mathrm{MEOH} \\
\mathrm{C} 6 \mathrm{H6} \\
\mathrm{C} 6 \mathrm{H60} \\
\mathrm{C} 7 \mathrm{H} 80 \\
\mathrm{C} 10 \mathrm{HB} \\
\mathrm{C} 14 \mathrm{HIO}\end{array}$ & $\begin{array}{r}17.11 \\
8.75 \\
21.37 \\
43.33 \\
8.43 \\
0.01 \\
0.62 \\
\\
0.1600 \\
0.0080 \\
0.1600 \\
0.0070 \\
0.0050\end{array}$ & $\begin{array}{r}11.22 \\
5.74 \\
19.82 \\
57.02 \\
5.53 \\
0.01 \\
0.41 \\
\\
0.1100 \\
0.0054 \\
0.1000 \\
0.0045 \\
0.0033\end{array}$ & $\begin{array}{r}12.81 \\
7.10 \\
19.41 \\
54.49 \\
6.15\end{array}$ & $\begin{array}{r}26.38 \\
12.28 \\
16.68 \\
44.33 \\
0.31\end{array}$ & $\begin{array}{r}66.38 \\
24.16 \\
5.98 \\
1.87 \\
1.56\end{array}$ & $\begin{array}{r}50.13 \\
7.71 \\
11.52 \\
0.67 \\
2.42\end{array}$ & $\begin{array}{r}72.39 \\
11.13 \\
12.92 \\
0.00 \\
0.09\end{array}$ \\
\hline $\begin{array}{l}\text { lb-mole/hr } \\
T, \text { deg F } \\
P, \text { psia } \\
H, B t u / l b \text {-mole } \\
H / C\end{array}$ & $\begin{array}{r}14221 \\
1526 \\
319 \\
-74330 \\
1.95\end{array}$ & $\begin{array}{r}21680 \\
1261 \\
319 \\
-86480 \\
1.95\end{array}$ & $\begin{array}{r}22130 \\
1196 \\
319 \\
-84730 \\
1.8\end{array}$ & $\begin{array}{r}24700 \\
1600 \\
319 \\
-66910 \\
2.15\end{array}$ & $\begin{array}{r}14570 \\
445 \\
750 \\
-21450 \\
2.75\end{array}$ & $\begin{array}{r}9400 \\
445 \\
750 \\
-45420 \\
6.5\end{array}$ & $\begin{array}{r}4359 \\
32 \\
740 \\
-28740 \\
6.5\end{array}$ \\
\hline
\end{tabular}




\section{Analysis of BCL Biomass Gasification Data}

The objective of modelling the BCL gasification data was to put the gasifier yield data in a form which could be input into the ASPEN process simulator. The experimental data were taken from the $1988 \mathrm{BCL}$ report to Pacific Northwest Laboratories (Feldmann etal, 1988). The following procedure was used to convert the experimental data:

1. The experimental results were input into a Lotus Spreadsheet and normalized to force a $100 \%$ material balance. The data are given in appendix 7 .

2. Yields and gas compositions were correlated using 1 inear least squares fits as polynomial function of temperature. The least squares results are given in Appendix 8. A summary of gasifier conditions, feed properties, and yield correlations is given in Appendix 9.

3. The yields correlations were then used to generate ASPEN input data. These input data a given in Appendix 10 . 


\section{ASPEN Model for the BCL Gasifier}

The BCL gasifier based system simulated for this study is given by Figures 3 and 4. The major process componenetsa simulated were:

1. Feed dryer

2. Gasifier with quench. The gasifier was modelled as a RYield reactor. The hot sand heating system was simulated by sand heating loop and char/tar/methanol combustion loop. Heat requirements were satidfied, but the hot sand stream was not actually mixed with the gasifier stream. A recycle gas stream was simulated in the same manner.

3. Reformer Compressor

4. Preformer/Reformer: The modules was simulated in the same manner as for the IGT case.

5. Acid gas removal and recycle: This module was simulated in the same manner as for the IGT case.

6. Methanol compressor: This module was simulated as three stage centrifugal compressor with intercooling.

7. Methanol synthesi/recovery: Theses modules were simulated in the same manner as for the IGT case.

8. Reformer furnace: This module was simulated in the same manner as for the IGT case.

9. Steam Generation: This module was simulated in the same manner as in the IGT case.

10. Electricity Generation: There was no electricity generation in this simulation.

A listing of the simulation program is given in Appendix 11: 


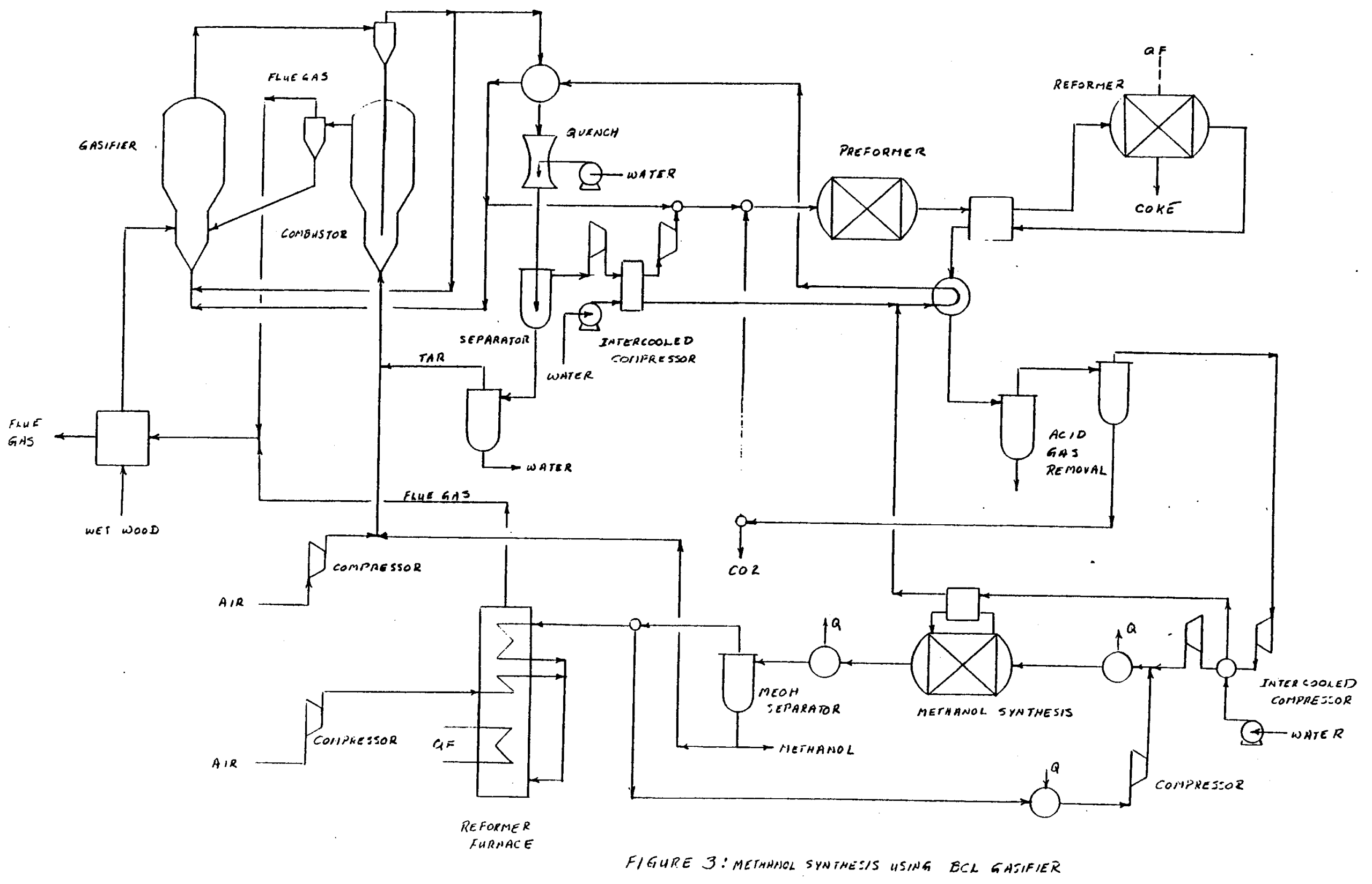




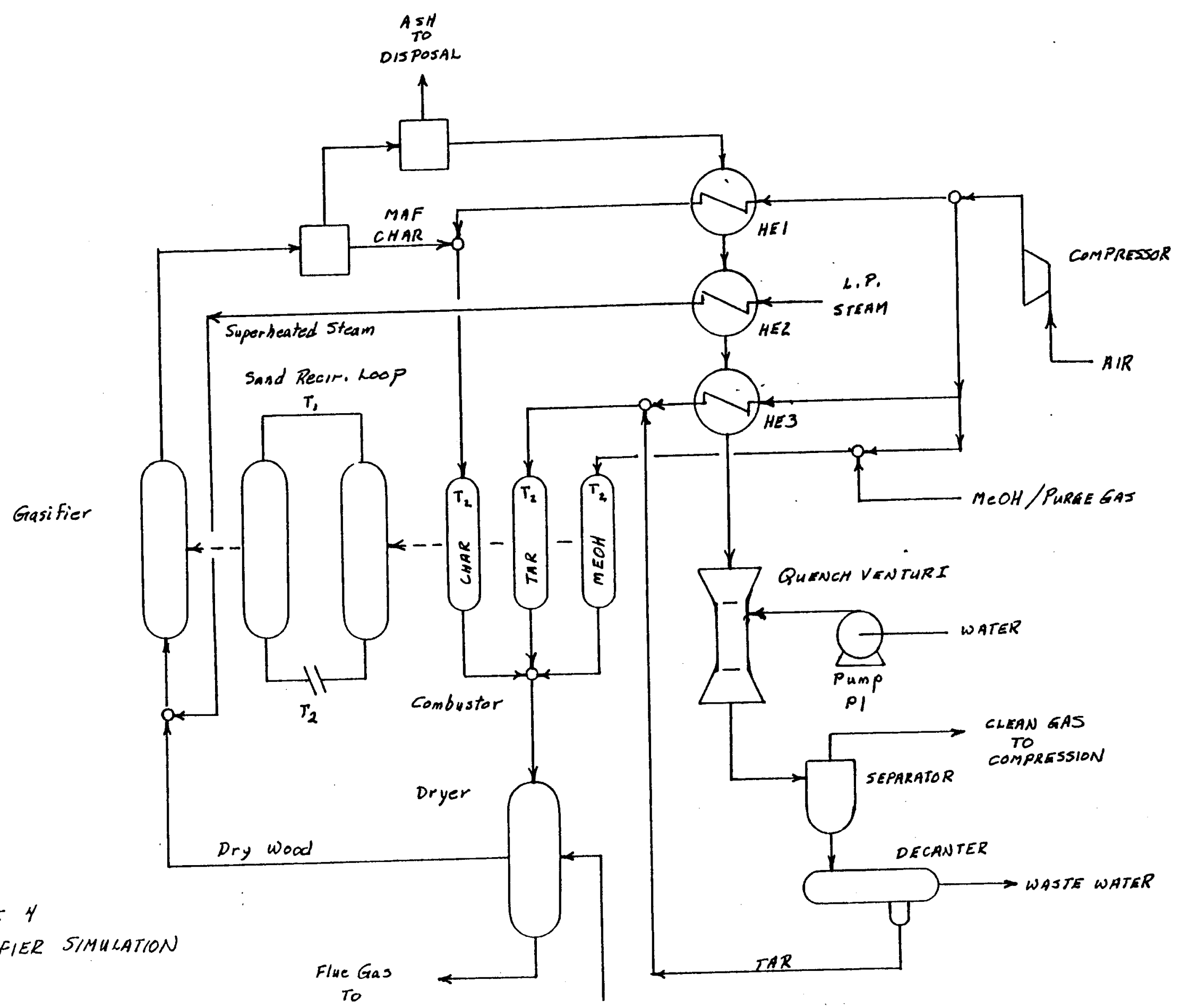




\section{Battelle Columbus Case Study}

The BCL simulation model was used to perform a simulation of base condition yields in which the raw gas leaving the gasifier was quenched and tars removed before entering the reformer train. The preliminary analysis is given below. In summary, the simulation indicates that 110.5 million gallons of methanol per year can be produced from 2000 dTPD wood. This production rate compares with 123.8 million gallons per year estimated in 1990. The differences are caused primarily by a more detailed study of dryer and steam generation heat requirements which have indicated that a larger purge gas stream from the methanol synthesis loop is required to meet process heat duty.

\section{System Configuration}
a. Dryer
b. Gasifier with quench and product gas recycle
c. Combustor
d. Reformer Compressor
e. Preformer
f. Reformer
g. Acid gas removal
f. Methanol synthesis compressor
g. Methanol synthesis
h. Reformer furnace

II. Feed

Total 1.333E $+091 \mathrm{~b} / \mathrm{yr} \quad \mathrm{HHV}=1.437 \mathrm{E}+10 \mathrm{Btu} / \mathrm{hr}$

Carbon $=0.6784 \mathrm{E}+09 \mathrm{ib} \cdot \mathrm{yr}$

III. Product
A. Methanol
$2840 \mathrm{lbmol} / \mathrm{hr}=110.5 \mathrm{MM} \mathrm{gal} / \mathrm{yr}=0.7281 \mathrm{E}+09 \mathrm{lb} / \mathrm{yr}$
$54.6 \%$ of dry wood
HHV $=64,290 \mathrm{Btu} / \mathrm{gal}=0.8878 \mathrm{E}+09 \mathrm{Btu} / \mathrm{hr}=61.78 \%$
Carbon $=0.2729 \mathrm{E}+09 \mathrm{Ib} / \mathrm{hr}=40.23 \%$
B. Carbon Dioxide
Overall $=0.4966 \mathrm{E}+091 \mathrm{~b} / \mathrm{yr}$
Carbon $=0.1355 \mathrm{E}+09 \mathrm{1b} / \mathrm{yr}$

IV. Carbon Balance Check

Methanol $\quad=0.2729 \mathrm{E}+09=40.23$

Carbon Dioxide $\quad=0.1355 \mathrm{E}+09=19.97$ 
$\begin{array}{ll}\text { Reformer Flue Gas } & =0.0633 \mathrm{E}+09=9.33 \\ \text { Combustor Flue Gas } & =0.1899 \mathrm{E}+09=27.85 \\ \text { AssOC. MeOH } & =0.0232 \mathrm{E}+09=3.43\end{array}$

The difference is caused by small lack of closure in gasifier carbon balance.

V. Drver

Temperature $=220^{\circ} \mathrm{F}$

Pressure $=14.7$ psia

$Q_{D}=0.00225 E+09 \mathrm{Btu} / \mathrm{hr}=0.16 \%$

VI. Gasifier

Temperature $=1675^{\circ} \mathrm{F}$

Pressure $=20$ psia

Gasifier Feed:

Dry wood

Moisture

Steam

$=166,667 \mathrm{lb} / \mathrm{hr}$

Solids/wood

Quench

Recycle Gas $=103,3001 \mathrm{~b} / \mathrm{hr}\left(1675^{\circ} \mathrm{F}\right)$

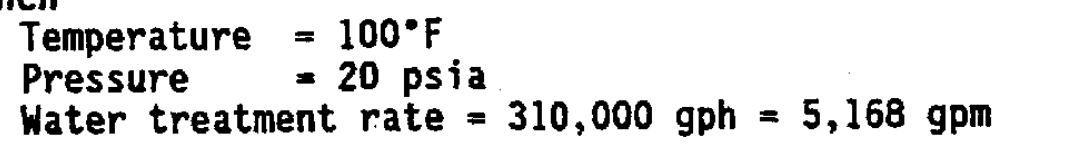

VII. Combustor

Circulation Material - SiO

Heat capacity $=0.2 \mathrm{Btu} / 1 \mathrm{~b} / \cdot \mathrm{F}$

Circulation Rate $=6,000,000 \mathrm{lb} / \mathrm{hr}$

Combustion feeds:

MAF char $=25,435 \mathrm{lb} / \mathrm{hr}$

Tar $=1,541 \mathrm{lb} / \mathrm{hr}$

Methanol $=801 \mathrm{~Tb} / \mathrm{hr}$

Air $=285,447 \mathrm{lb} / \mathrm{hr}$

Compressor Requirements, 25 psia, 2,677 hp

Compressor Cost estimate

Reference: Garrett, D.E., Chemical Engineering Economics, using figure on page 271

4 - 900 hp compressors $(3+1$ spare)

Purchase Price $=\$ 59,000$ (1987\$)

Module factor $=2.6$

No. Units $=4$

Installed Cost $=59,000 * 2.6 * 4=\$ 613,600$

VIII. Reformer Compressor 
Modelled as a 4 stage centrifugal compressor with intercooling.

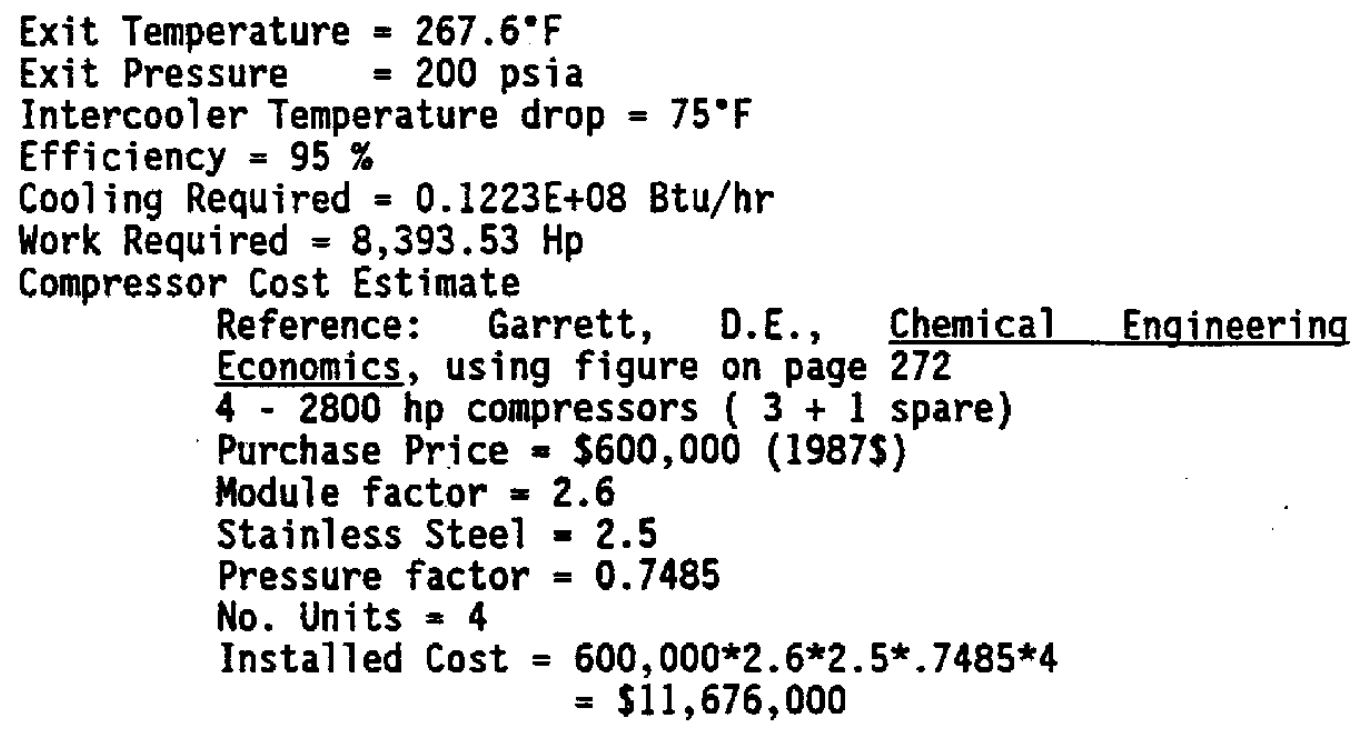

IX. Reformer Loop

A. In]et Conditions

Temperature $=267.6^{\circ} \mathrm{F}$

Pressure $=200$ psia

Steam Added $=8,000 \mathrm{lbmol} / \mathrm{hr}$

B. Preformer - Converts non-methane hydrocarbons

Temperature $=1000^{\circ} \mathrm{F}$

C. Preheater

Temperature Out $=1571^{\circ} \mathrm{F}$

$Q=0.0937 E+09$ Btu/hr

$A=16,152 \mathrm{ft}^{2}$

D. Reformer

Temperature $=1600^{\circ}$

Pressure $=200 \mathrm{psia}$

$Q=-0.1112 E+09 \mathrm{Btu} / \mathrm{hr}$

Gas Composition

$\begin{array}{lcc}\text { Comp } & \text { Mole \% } & \text { Mole \% dry } \\ \text { H2 } & 34.04 & 50.24 \\ \text { CO } & 18.79 & 27.73 \\ \text { CO2 } & 14.38 & 21.22 \\ \text { H2O } & 32.24 & -- \\ \text { CH4 } & 0.55 & 0.81\end{array}$

E. $\mathrm{CO}_{2}$ Recycle 
Product $/$ Recycle $=1410 / 1154=1.22$

X. Methanol Recycle

A. Methanol Compressor

Pin $=200 \mathrm{psia}$
Pout $=750 \mathrm{psia}$
Tin $=425 . \mathrm{F}$
Tout $=714.4{ }^{\circ} \mathrm{F}$
Efficiency $=0.95$

Modelled as 3 stage centrifugal compressor with intercooling.

Work Required $=11,727.3 \mathrm{Hp}$

Heat Removed $=0.007599 \mathrm{E}+09 \mathrm{Btu} / \mathrm{hr}$

Cost Estimate:

Reference: Garrett, D.E., Chemical Engineering Economics, using figure on page 272

No. Comp $=4(3+1$ spare $) 3940 \mathrm{Hp}$ ea

Base Cost $(1987 \$)=\$ 605,000$

Module Factor $=2.6$

Stainless Steel $=2.5$

Pressure factor $=0.9495$

Cost $=605,000 * 4 * 2.6 * 2.5 * 0.9495=\$ 14,935,600$

B. Heater 5 (Recycle Preheat - to el iminate condensation in recycle compressor) $Q=0.002901 \mathrm{E}+09 \mathrm{Btu} / \mathrm{hr}$

C. Recycle Compressor: $22.3 \mathrm{Hp}$

D. Htr 6 (Used to balance compressor preheat)

$Q=0.01326 \mathrm{E}+09 \mathrm{Btu} / \mathrm{hr}>.002901 \mathrm{E}+09$ (ok)

E. Methanol Synthesis

Temperature $=445^{\circ}$

Pressure $=750$ psia

$Q=-0.1282 E+09 \mathrm{Btu} / \mathrm{hr}$

Q used for steam production

F. Product cooling $Q=0.0826 \mathrm{E}+09 \mathrm{Btu} / \mathrm{hr}$

Not used in present case

G. Methanol Separation

$Q=0.0003882 \mathrm{E}+09 \mathrm{Btu} / \mathrm{hr}$

Raw Product Liquid Composition

$\begin{array}{lcc}\text { Comp } & \text { lbmole/hr } & \text { Mole \% } \\ \text { H2 } & 2.82 & 0.09 \\ \text { CO } & 3.09 & 0.10 \\ \text { CO2 } & 233.45 & 7.46 \\ \text { H2O } & 18.41 & 0.58 \\ \text { CH4 } & 5.28 & 0.16 \\ \text { MEOH } & 2865.25 & 91.59\end{array}$


H. Recycle/Purge $=3247 / 1599=2.03$

XI. Reformer Combustor

A. Air Compressor

Work Required $=832.3 \mathrm{Hp}$

Air Rate $=5500 \mathrm{lb}-\mathrm{mole} / \mathrm{hr}$

Exit Temperature $=135.1^{\circ} \mathrm{F}$

Pressure $=20$ psia

Cost

Reference: Garrett, D.E., Chemical Engineering

Economics, using figure on page 271

No. Units $=3(2+1$ spare $)$

Base Cost $=\$ 46,000(1987 \$)$

Module factor $=2.6$

Cost $=46,000 * 2.6 * 3=\$ 358,800$

B. Air Preheater

$$
\begin{aligned}
& Q=0.04198 \mathrm{E}+09 \mathrm{Btu} / \mathrm{hr} \\
& A=425 \mathrm{ft}^{2}
\end{aligned}
$$

C. Feed Gas Preheater

$$
\begin{aligned}
& Q=0.005309 \mathrm{E}+09 \mathrm{Btu} / \mathrm{hr} \\
& A=55.4 \mathrm{ft}^{2}
\end{aligned}
$$

D. Combustor

$T=1730^{\circ} \mathrm{F}>1700^{\circ} \mathrm{F}$ (Assumed $100^{\circ} \mathrm{F}$ approach temperature in reformer furnace.)

Excess heat $=0.001752 \mathrm{E}+09 \mathrm{Btu} / \mathrm{hr}$

XII. Steam Generation

$11,750 \mathrm{lb}-\mathrm{mol} / \mathrm{hr}$ at $1036^{\circ} \mathrm{F}, 200 \mathrm{psia}$

Steam Required:

$$
\begin{array}{ll}
\text { Gasifier: } & 3,684 \mathrm{lbmol} / \mathrm{hr} \\
\text { Reformer: } & 8,0001 \mathrm{bmol} / \mathrm{hr} \\
\text { Total: } & 11,684 \mathrm{lbmol} / \mathrm{hr}
\end{array}
$$

XIII. Preliminary Electrical

\begin{tabular}{ll} 
Quench Pump & $17.61 \mathrm{Hp}$ \\
Steam Gen Pump & $\mathbf{4 7 . 6 0}$ \\
Gasifier Air Compressors & 2,677 \\
Reformer Compressor & 8,394 \\
Methanol Compressor & 11,727 \\
Reformer Air Compressor & 832 \\
\multicolumn{1}{l}{ Subtotal } & $23,695 \mathrm{Hp}=17.7 \mathrm{MW}$
\end{tabular}


To Be added:

Dryer/Conveyer/Feeder

Acid Gas

Misc 
Gas Composition, Major Streams

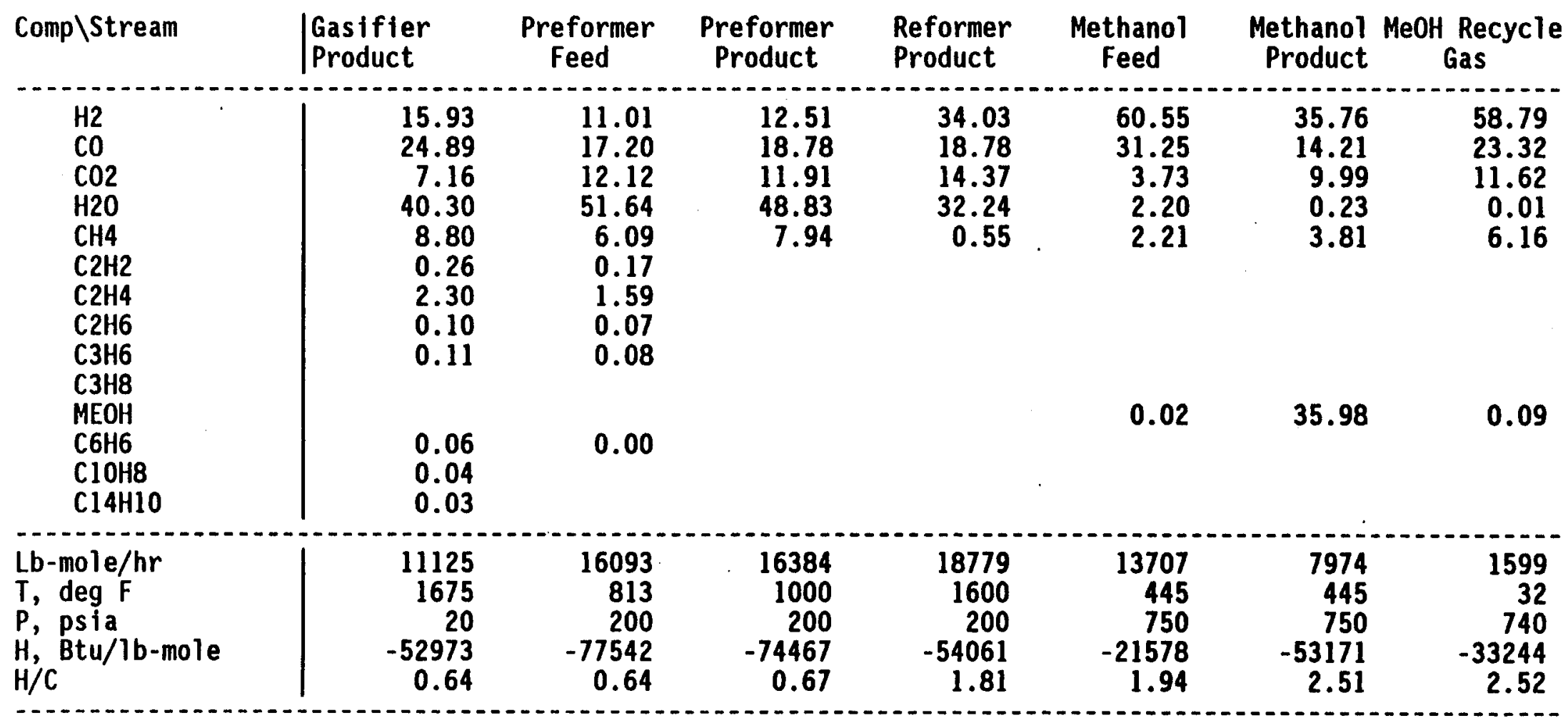




\section{References}

Bain, R.L. "Methanol from Biomass: Assessment of Production Costs," Hawaji Natural Energy Institute Renewable Transportation Alternatives Workshop, Honolulu, HI, Jan 9-11, 1991.

Chem Systems "Assessment of Costs and Benefits of Flexible and Alternative Fuel Use in the U.S. Transportation Sector", by Chem Systems, Tarrytown, NY, for the U.S. Department of Energy, Washington, D.C., Report No. DOE/PE--0093, 46 pp, November 1989.

Evans, R.J., Knight, R.A., Onischak, M., and S.P. Babu, "Development of Biomass Gasification to Produce Substitute Fuels, Prepared by the Institute of Gas Technology, Chicago, Illinois, for the Pacific Northwest Laboratory, Richland, WA, PNL- Report No. PNL-6518, March 1988.

Feldmann, H.F., Paisley, M.A., Appelbaum, H.R., and D.R. Taylor, "Conversion of Forest Residues to a Methane-Rich Gas in a High- Throughput Gasifier", prepared by Battel Te Columbus Division, Columbus, Ohio, for the Pacific Northwest Laboratory, Richland, WA, PNL Report no. PNL-6570, May 1988.

Flanigan V.J., O.C. Sitton and W.E. Huang, "The Development of a 20-inch Indirect Fired Fluidized Bed Gasifier," University of Missouri-Rolla for Pacific Northwest Laboratory, PNL-6520, Richland, WA, March 1988.

Graboski, M. and R.L. Bain, "Properties of Biomass Relevant to Gasification," Chapter 3 of A Survey of Biomass Gasification - Volume?, Solar Energy Research Institute, SERI/TR-33-239, Golden, CO, JuTy 1979.

Keenan, J.D., "Biochemical Sources of Fuel," in Energy Technology Handbook, ed D. M. Considine, McGraw-Hill Book Co., New York, NY, pp 4-14 to $4-15,1977$.

Mahajan, D., Sapienza, R.S., and T.E O'Hare, "Bench-Scale Laboratory Result - Novel Methanol Catalyst," Thirteenth Annual EPRI Conf, Fuel Science and Conversion, EPRI, Palo Alto, CA, 1989.

Manufacturing and Technology Conversion International, Inc. "Testing of an Advanced Thermochemical Conversion Reactor System," Pacific Northwest Laboratory, Richland, WA, PNL-7245, January 1990.

Philip, R.J. "Methanol Production from Biomass," National Research Council of Canada, NRCC No. 27143, Ottawa, Ontario, Canada, pp 29-32, August 1986.

Reed, T.B., B. Levie, and M.S. Graboski, "Fundamentals, Development and Scale-up of the Air-Oxygen Stratified Downdraft Gasifier, " Pacific Northwest laboratory, Richland, WA, PNL-6600, June 1988.

Rensfelt, E. and C. Ekstrom, "Fuel from Municipal Waste in an Integrated Circulating Fluid-Bed Gasification/Gas Cleaning Process," In Energy form 
Biomass and Wastes XI, ed D.L. Klass, Institute of Gas Technology, Chicago, IL, pp 891-906, 1989.

Simulation Sciences, Aspen/SP Users Manual, Denver, CO, 1990.

Studer, D.W.; Brown, D.M., Henderson, J.L. and T.H. Hsiung, "Status of the Development of Methanol Synthesis by the "LPMEOH'Process", DOE Indirect Liquefaction Contractors' Review Meeting, Pittsbugh, PA 13-15 Nov, 1989. 
APPENDIX 1 
IGT GASIFIER DATA

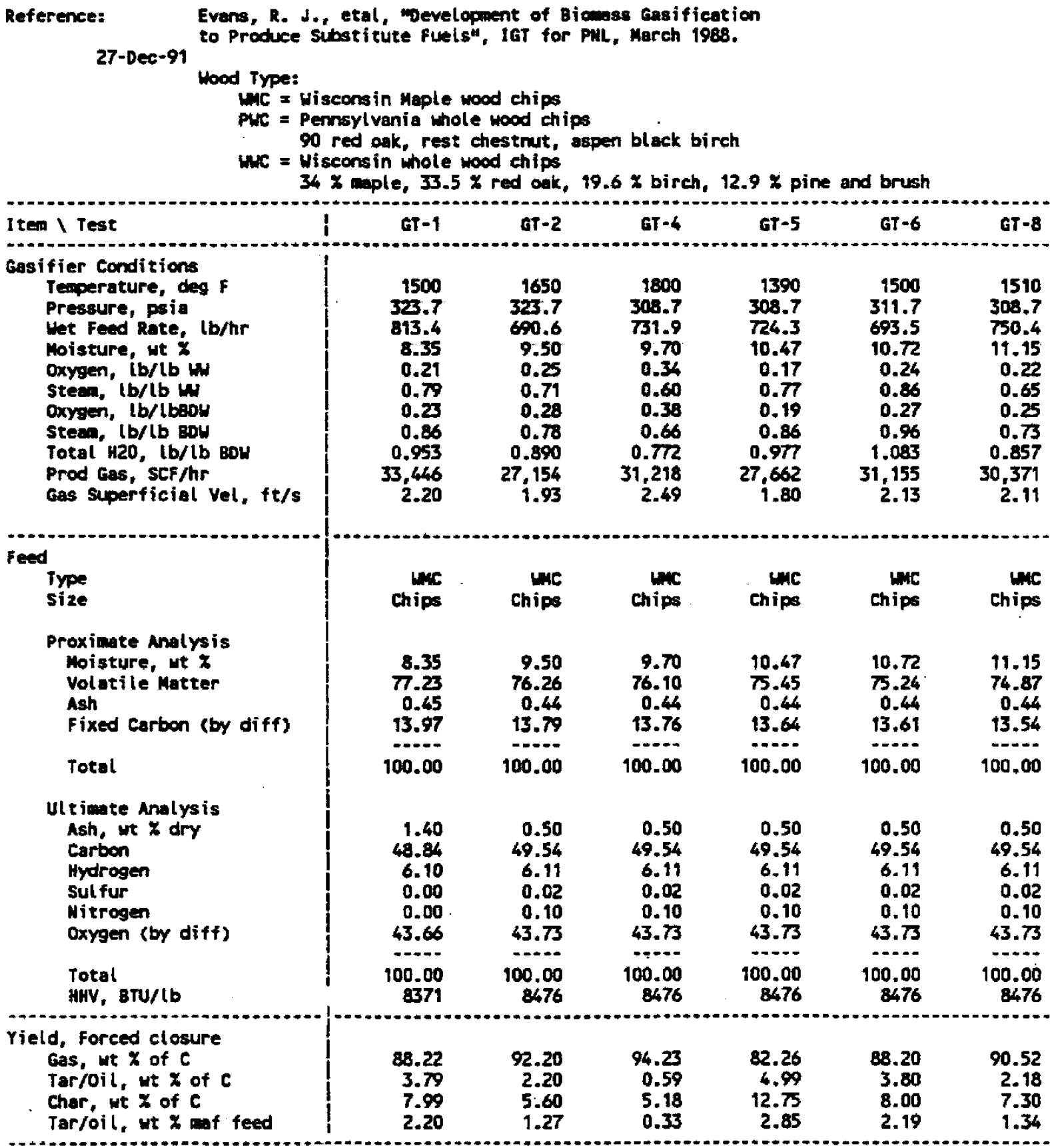




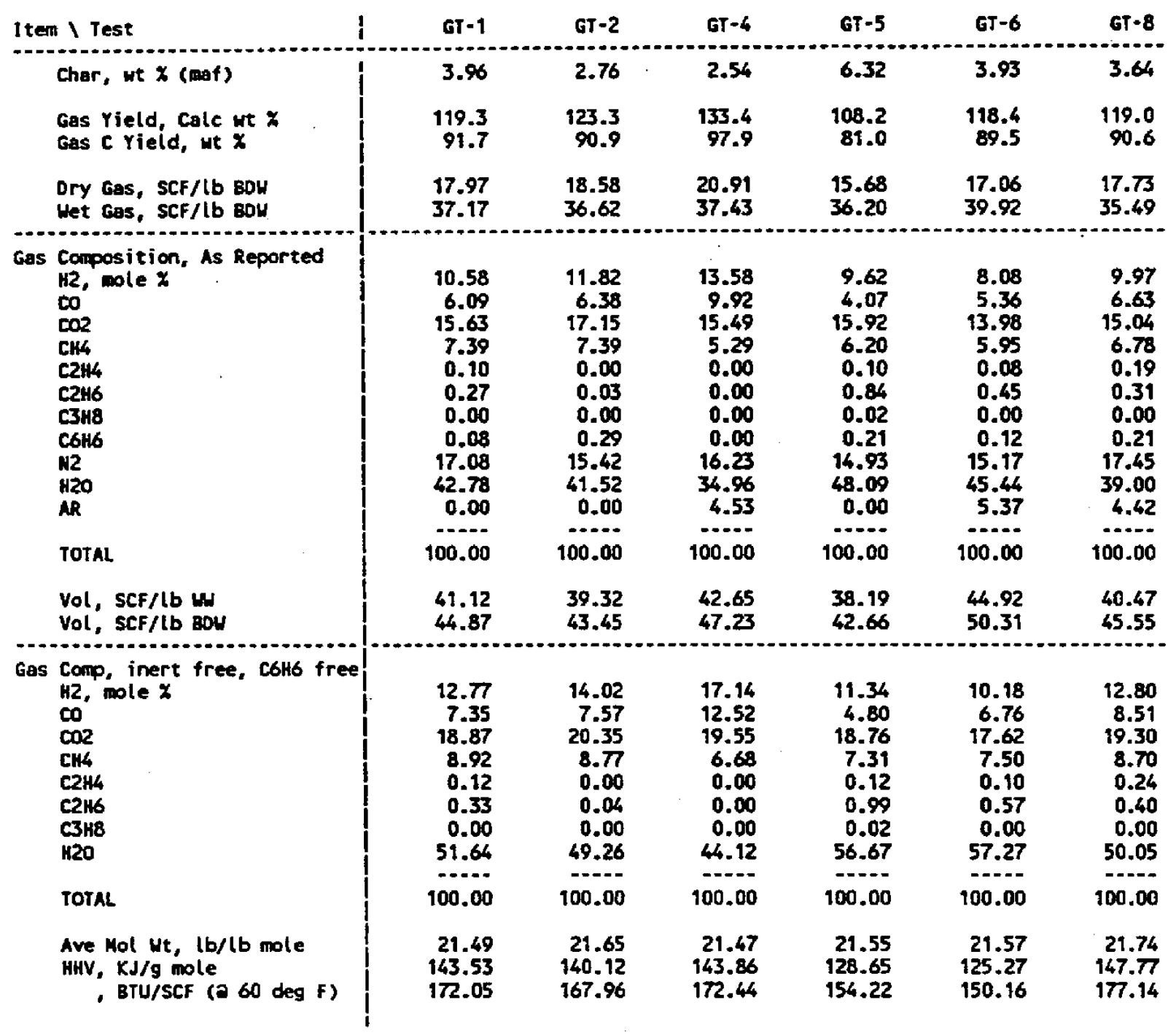




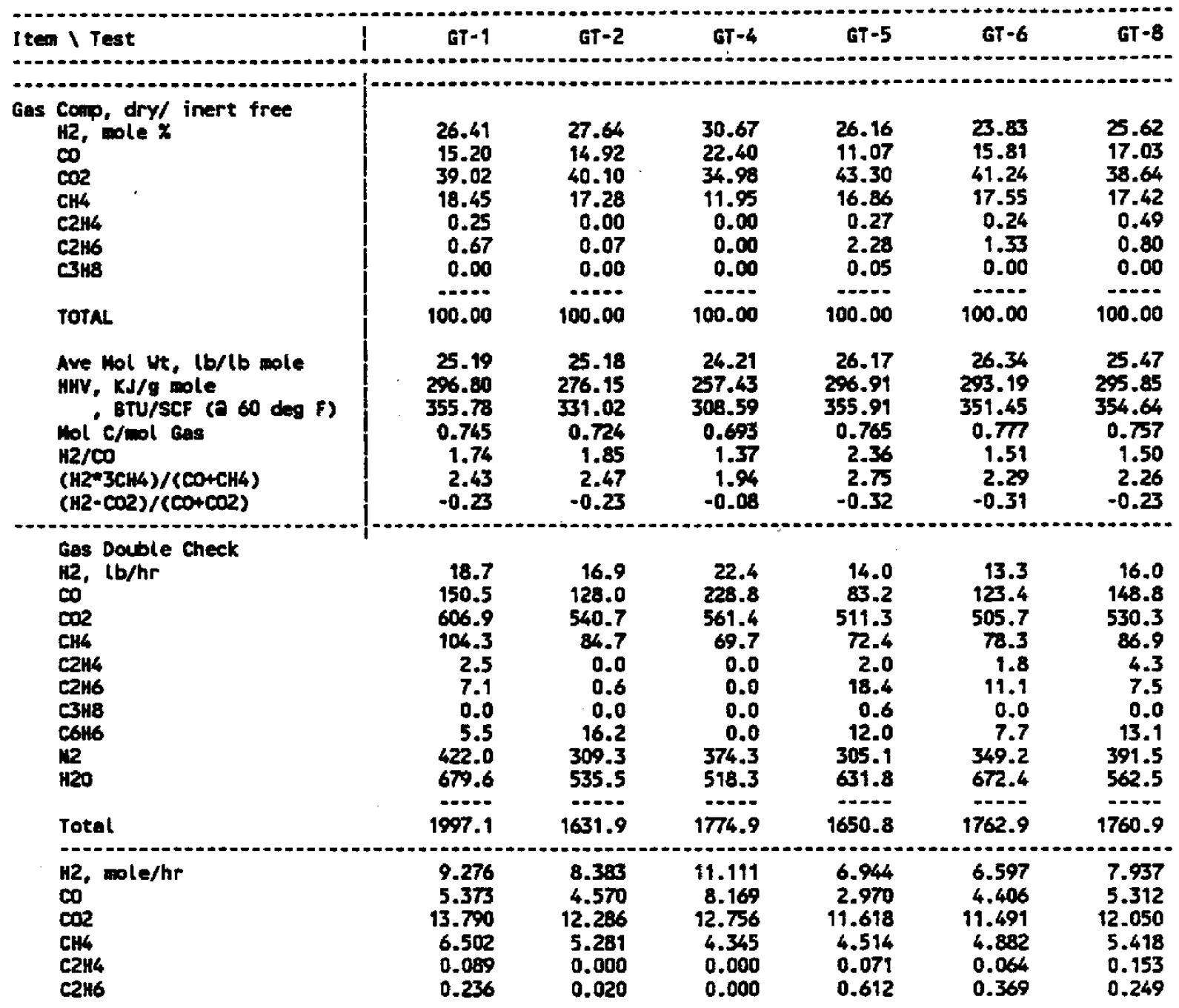




\begin{tabular}{|c|c|c|c|c|c|c|}
\hline Iten I Test & $G T-1$ & GT-2 & GT-4 & $6 T-5$ & GT-6 & GT-8 \\
\hline $\begin{array}{l}\text { C3H8 } \\
\text { C6H6 } \\
\text { M2 } \\
H 2 O\end{array}$ & $\begin{array}{r}0.000 \\
0.070 \\
15.061 \\
37.722\end{array}$ & $\begin{array}{r}0.000 \\
0.207 \\
11.039 \\
29.724\end{array}$ & $\begin{array}{r}0.000 \\
0.000 \\
13.358 \\
28.769\end{array}$ & $\begin{array}{r}0.014 \\
0.154 \\
10.889 \\
35.069\end{array}$ & $\begin{array}{r}0.000 \\
0.099 \\
12.463 \\
37.322\end{array}$ & $\begin{array}{r}0.000 \\
0.168 \\
13.972 \\
31.222\end{array}$ \\
\hline Total & 88.120 & 71.509 & 78.508 & 72.854 & 77.692 & 76.481 \\
\hline $\begin{array}{l}\text { lb bolw/hr } \\
\text { Vol, scf/ lb bdu } \\
\text { M2 Free vol } \\
\text { M2, csils free vol } \\
\text { Dry Ges Vol }\end{array}$ & $\begin{array}{l}745.5 \\
44.85 \\
38.09 \\
38.06 \\
21.14\end{array}$ & $\begin{array}{r}625 \\
43.41 \\
38.62 \\
38.53 \\
25.62\end{array}$ & $\begin{array}{l}660.9 \\
45.07 \\
39.05 \\
39.05 \\
26.08\end{array}$ & $\begin{array}{l}648.5 \\
42.62 \\
37.98 \\
37.92 \\
22.97\end{array}$ & $\begin{array}{l}619.2 \\
47.60 \\
41.67 \\
41.62 \\
23.86\end{array}$ & $\begin{array}{l}666.7 \\
43.52 \\
37.44 \\
37.37 \\
23.78\end{array}$ \\
\hline $\begin{array}{l}\text { H2, mole X } \\
\text { CO } \\
\text { CO2 } \\
\text { CH4 } \\
\text { C2144 } \\
0216 \\
\text { C3H8 } \\
\text { C6H6 } \\
\text { H2 } \\
H 20\end{array}$ & $\begin{array}{r}10.53 \\
6.10 \\
15.65 \\
7.38 \\
0.10 \\
0.27 \\
0.00 \\
0.08 \\
17.09 \\
42.81\end{array}$ & $\begin{array}{r}11.72 \\
6.39 \\
17.18 \\
7.38 \\
0.00 \\
0.03 \\
0.00 \\
0.29 \\
15.44 \\
41.57\end{array}$ & $\begin{array}{r}14.15 \\
10.40 \\
16.25 \\
5.53 \\
0.00 \\
0.00 \\
0.00 \\
0.00 \\
17.02 \\
36.64\end{array}$ & $\begin{array}{r}9.53 \\
4.08 \\
15.95 \\
6.20 \\
0.10 \\
0.84 \\
0.02 \\
0.21 \\
14.95 \\
48.14\end{array}$ & $\begin{array}{r}8.49 \\
5.67 \\
14.79 \\
6.28 \\
0.08 \\
0.48 \\
0.00 \\
0.13 \\
16.04 \\
48.04\end{array}$ & $\begin{array}{r}10.38 \\
6.95 \\
15.75 \\
7.08 \\
0.20 \\
0.33 \\
0.00 \\
0.22 \\
18.27 \\
40.82\end{array}$ \\
\hline TOTAL & 100.00 & 100.00 & 100.00 & 100.00 & 100.00 & 100.00 \\
\hline $\begin{array}{l}\mathrm{H2} \text {, mole } \mathrm{X} \\
\mathrm{CO} \\
\mathrm{CO2} \\
\mathrm{CH4} \\
\mathrm{C2H4} \\
\mathrm{C2H6} \\
\mathrm{C} \mathrm{HH} \\
\mathrm{H} 2 \mathrm{O}\end{array}$ & \begin{tabular}{r}
12.71 \\
7.36 \\
18.89 \\
8.91 \\
0.12 \\
0.32 \\
0.00 \\
51.68 \\
\hdashline-100 \\
100.00
\end{tabular} & $\begin{array}{r}13.91 \\
7.58 \\
20.39 \\
8.76 \\
0.00 \\
0.03 \\
0.00 \\
49.32 \\
100.00\end{array}$ & \begin{tabular}{r}
17.05 \\
12.54 \\
19.58 \\
6.67 \\
0.00 \\
0.00 \\
0.00 \\
44.16 \\
\hdashline 0.00 \\
100.00
\end{tabular} & $\begin{array}{r}11.23 \\
4.81 \\
18.80 \\
7.30 \\
0.12 \\
0.99 \\
0.02 \\
56.73 \\
100.00\end{array}$ & $\begin{array}{r}10.13 \\
6.76 \\
17.64 \\
7.50 \\
0.10 \\
0.57 \\
0.00 \\
57.30 \\
100.00\end{array}$ & $\begin{array}{r}12.73 \\
8.52 \\
19.33 \\
8.69 \\
0.25 \\
0.40 \\
0.00 \\
50.08 \\
\hdashline-100.00\end{array}$ \\
\hline
\end{tabular}




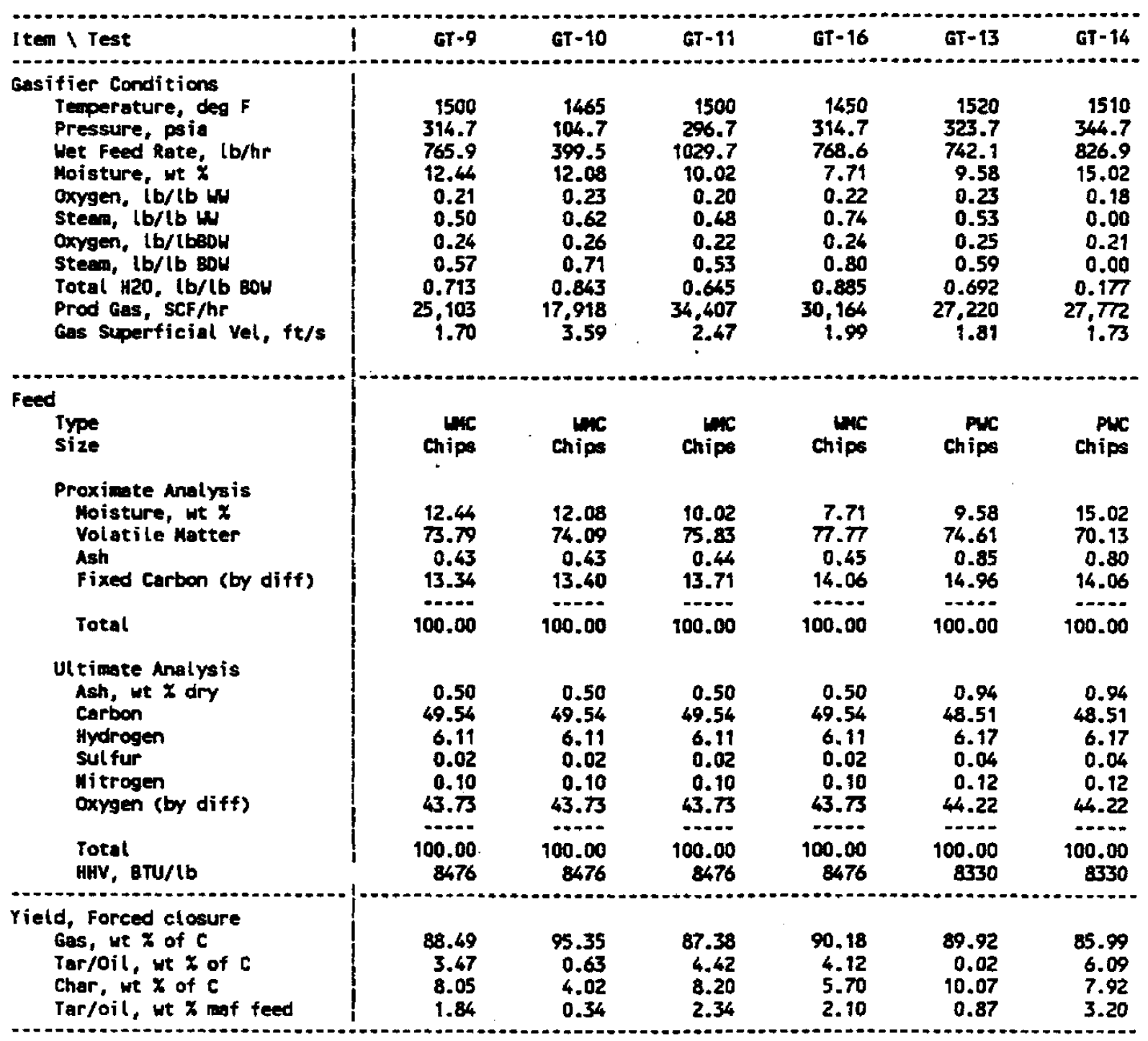




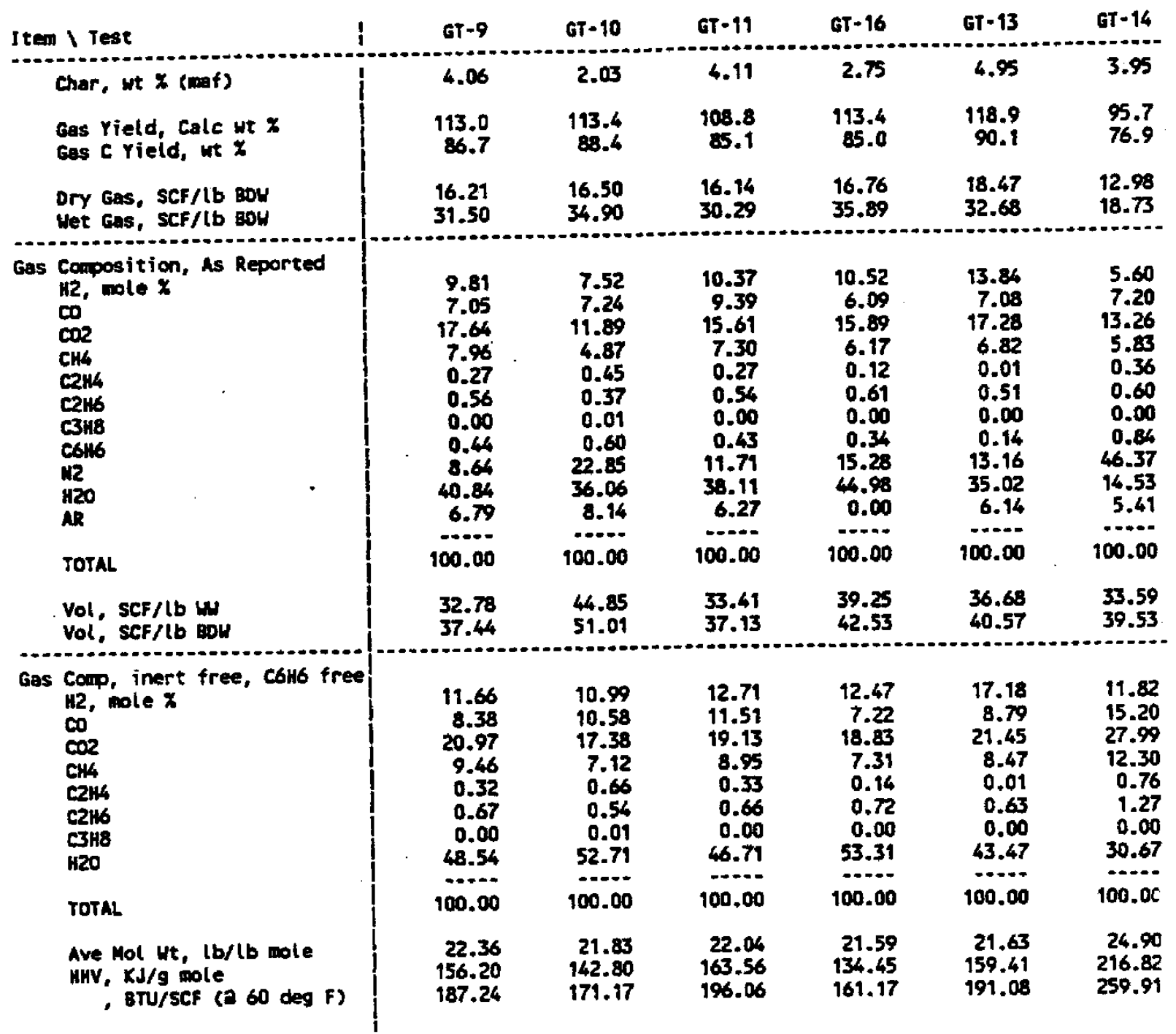




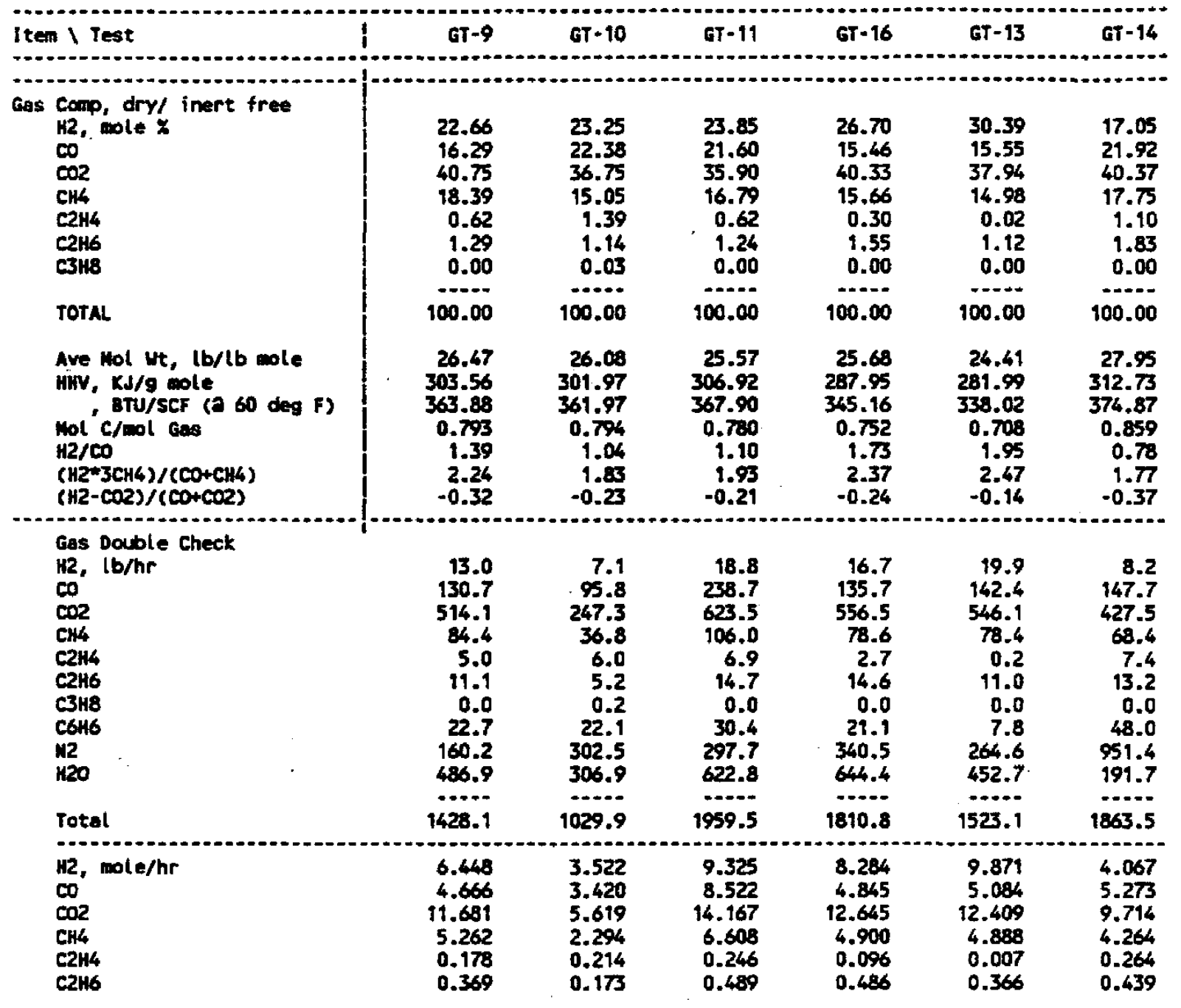




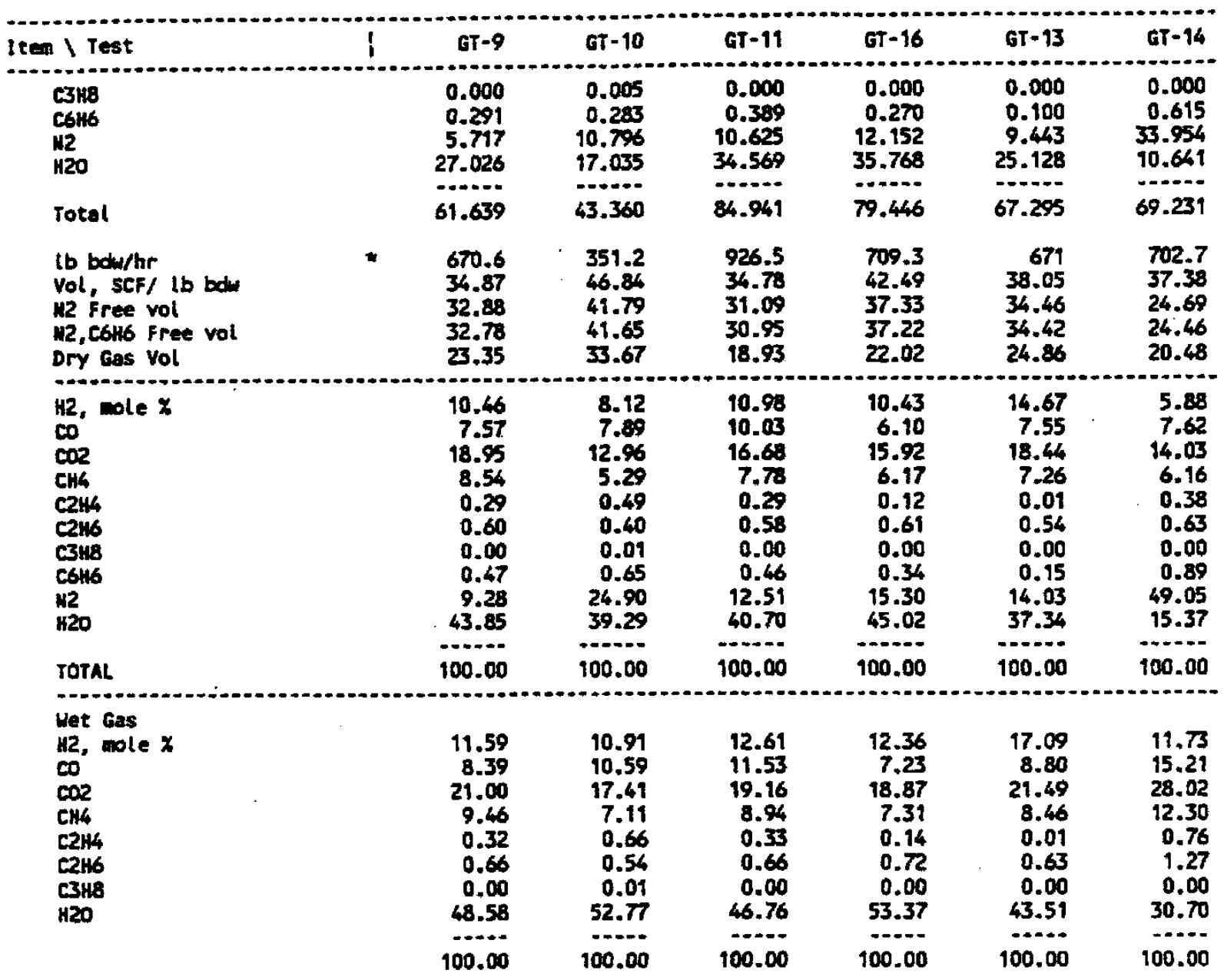




\begin{tabular}{|c|c|c|c|c|c|c|}
\hline $\ln 1$ & $G T \cdot 15$ & $0 T-17$ & T12-1 & T12-2 & $T 3 D-1 a$ & $T 30-1 b$ \\
\hline 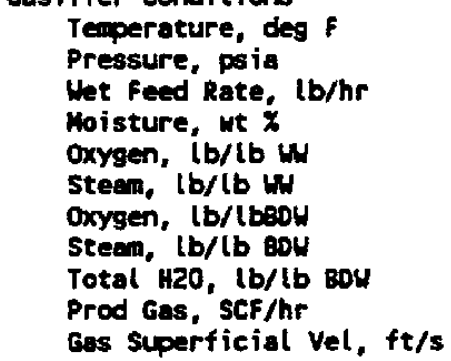 & $\begin{array}{r}1520 \\
320.7 \\
673.4 \\
14.47 \\
0.25 \\
0.41 \\
0.29 \\
0.48 \\
0.649 \\
28,271 \\
1.90\end{array}$ & $\begin{array}{r}1530 \\
320.7 \\
648.8 \\
26.74 \\
0.33 \\
0.46 \\
0.45 \\
0.63 \\
0.993 \\
33,016 \\
2.23\end{array}$ & $\begin{array}{r}1530 \\
314.7 \\
643.9 \\
4.94 \\
0.25 \\
0.61 \\
0.26 \\
0.64 \\
0.694 \\
24.603 \\
1.69\end{array}$ & $\begin{array}{r}1500 \\
116.7 \\
352.9 \\
7.72 \\
0.26 \\
0.69 \\
0.28 \\
0.75 \\
0.831 \\
15.693 \\
2.87\end{array}$ & $\begin{array}{r}1538 \\
323.7 \\
427.5 \\
10.80 \\
0.26 \\
1.18 \\
0.29 \\
1.32 \\
1.444 \\
23,003 \\
1.55\end{array}$ & $\begin{array}{r}1562 \\
323.7 \\
748 \\
10.80 \\
0.23 \\
0.63 \\
0.26 \\
0.71 \\
0.827 \\
27,695 \\
1.88\end{array}$ \\
\hline $\begin{array}{l}\text { Type } \\
\text { Size }\end{array}$ & $\begin{array}{l}\text { Pur } \\
\text { Chips }\end{array}$ & $\begin{array}{r}\text { PuF } \\
\text { Chips }\end{array}$ & chips & $\begin{array}{r}\text { Mx } \\
\text { Chips }\end{array}$ & $\begin{array}{l}\text { me } \\
\text { Chips }\end{array}$ & Muc \\
\hline $\begin{array}{l}\text { Proximate Analysis } \\
\text { Moisture, wt } \% \\
\text { Volatile Matter } \\
\text { Ash } \\
\text { Fixed Carbon (by diff) } \\
\text { Total }\end{array}$ & $\begin{array}{r}14.47 \\
70.58 \\
0.80 \\
14.15 \\
\hdashline 100.00\end{array}$ & $\begin{array}{r}26.74 \\
60.45 \\
0.69 \\
12.12 \\
100.00\end{array}$ & $\begin{array}{r}4.94 \\
79.39 \\
0.77 \\
16.90 \\
100.00\end{array}$ & $\begin{array}{r}7.72 \\
77.07 \\
0.75 \\
14.46 \\
100.00\end{array}$ & $\begin{array}{r}10.80 \\
76.50 \\
0.72 \\
13.98 \\
100.00\end{array}$ & $\begin{array}{r}10.80 \\
74.50 \\
0.72 \\
13.98 \\
\hdashline-100.00\end{array}$ \\
\hline $\begin{array}{l}\text { Uttimate Analysis } \\
\text { Ash, wt } x \text { dry } \\
\text { Carbon } \\
\text { Hydrogen } \\
\text { Sul fur } \\
\text { Nitrogen } \\
\text { Oxygen (by diff) }\end{array}$ & $\begin{array}{r}0.94 \\
48.51 \\
6.17 \\
0.04 \\
0.12 \\
44.22\end{array}$ & $\begin{array}{r}0.94 \\
48.51 \\
6.17 \\
0.04 \\
0.12 \\
46.22\end{array}$ & $\begin{array}{r}0.82 \\
48.40 \\
6.31 \\
0.03 \\
0.21 \\
46.23\end{array}$ & $\begin{array}{r}0.82 \\
48.40 \\
6.31 \\
0.03 \\
0.21 \\
44.23\end{array}$ & $\begin{array}{r}0.82 \\
48.40 \\
6.31 \\
0.03 \\
0.21 \\
44.23\end{array}$ & $\begin{array}{r}0.82 \\
48.40 \\
6.31 \\
0.03 \\
0.21 \\
44.23\end{array}$ \\
\hline $\begin{array}{l}\text { Total } \\
\text { HHV, BTU/Lb }\end{array}$ & $\begin{array}{r}100.00 \\
8330\end{array}$ & $\begin{array}{r}100.00 \\
8330\end{array}$ & $\begin{array}{r}100.00 \\
8389\end{array}$ & $\begin{array}{r}100.00 \\
8389\end{array}$ & $\begin{array}{r}100.00 \\
8389\end{array}$ & $\begin{array}{r}100.00 \\
8389\end{array}$ \\
\hline $\begin{array}{l}\text { Yield, Forced closure } \\
\text { Gas, wt } X \text { of } C \\
\text { Tarjoil, wt } X \text { of } C \\
\text { Char, wt } X \text { of } C \\
\text { Tarjoil, we } X \text { maf feed }\end{array}$ & $\begin{array}{r}90.24 \\
4.28 \\
5.48 \\
2.26\end{array}$ & $\begin{array}{r}96.68 \\
0.00 \\
3.32 \\
0.00\end{array}$ & $\begin{array}{r}90.18 \\
5.46 \\
4.36 \\
2.97\end{array}$ & $\begin{array}{r}87.37 \\
5.33 \\
7.30 \\
2.88\end{array}$ & $\begin{array}{r}92.95 \\
3.07 \\
3.98 \\
1.61\end{array}$ & $\begin{array}{r}93.79 \\
2.98 \\
3.23 \\
1.57\end{array}$ \\
\hline
\end{tabular}




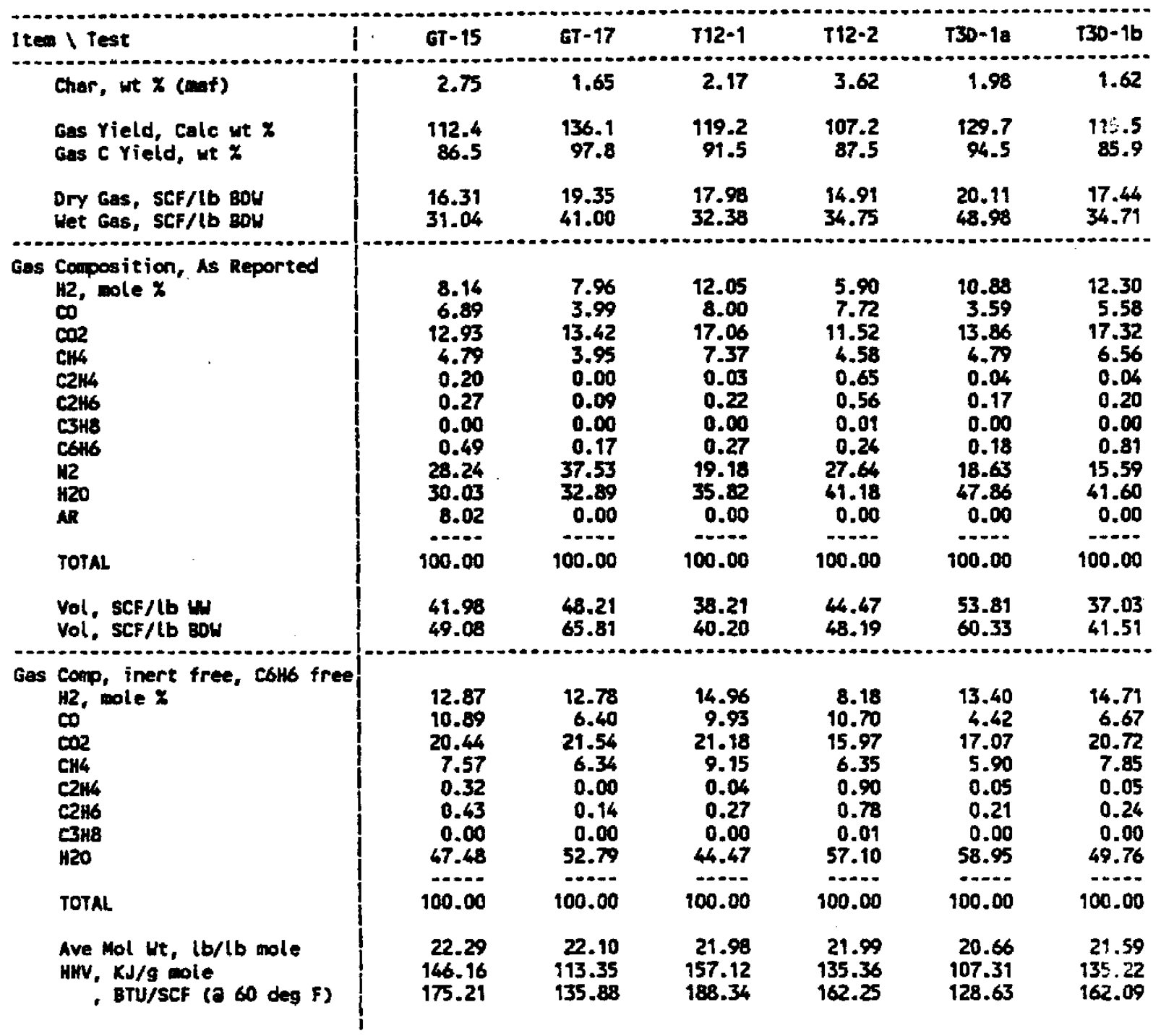




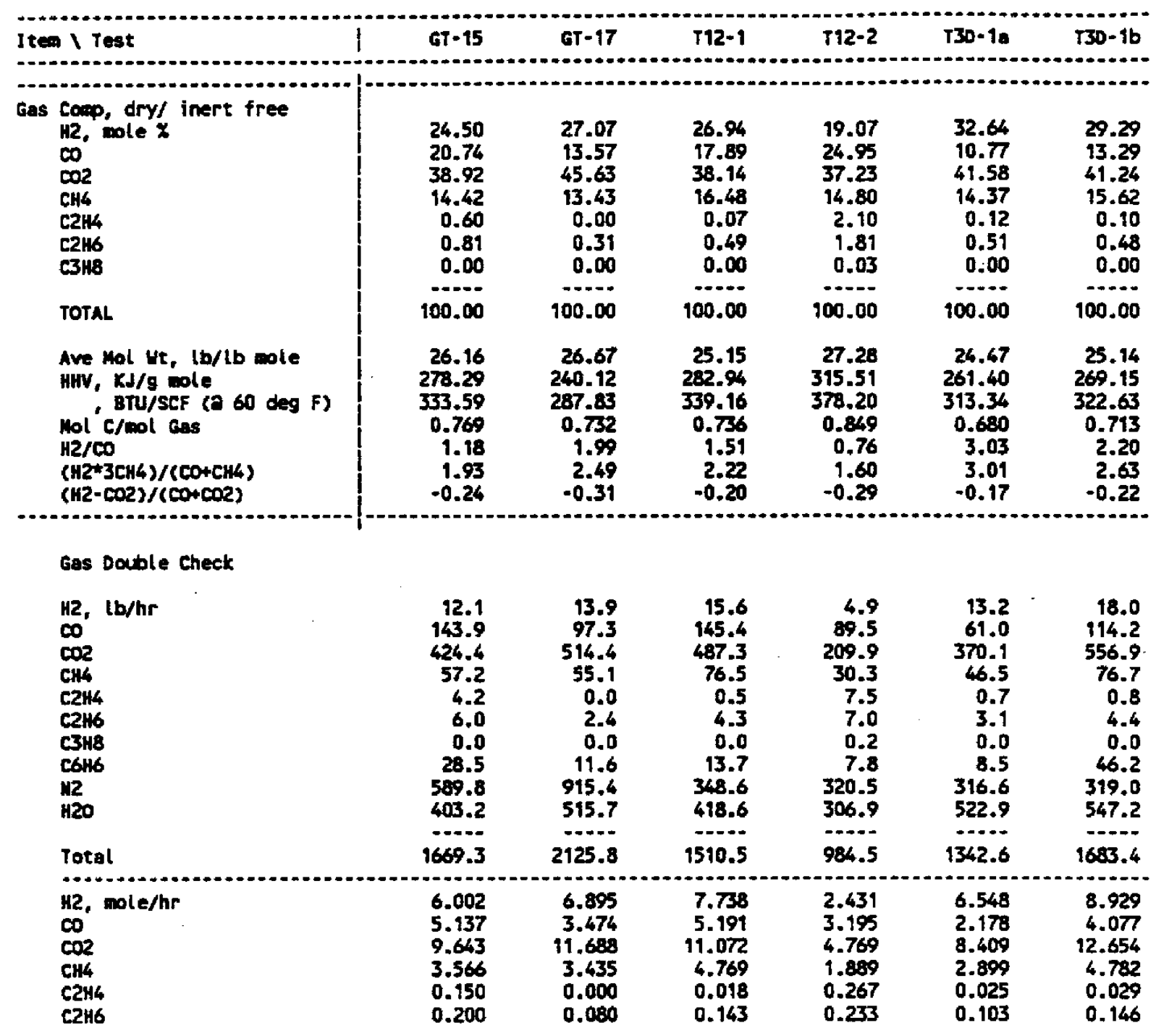




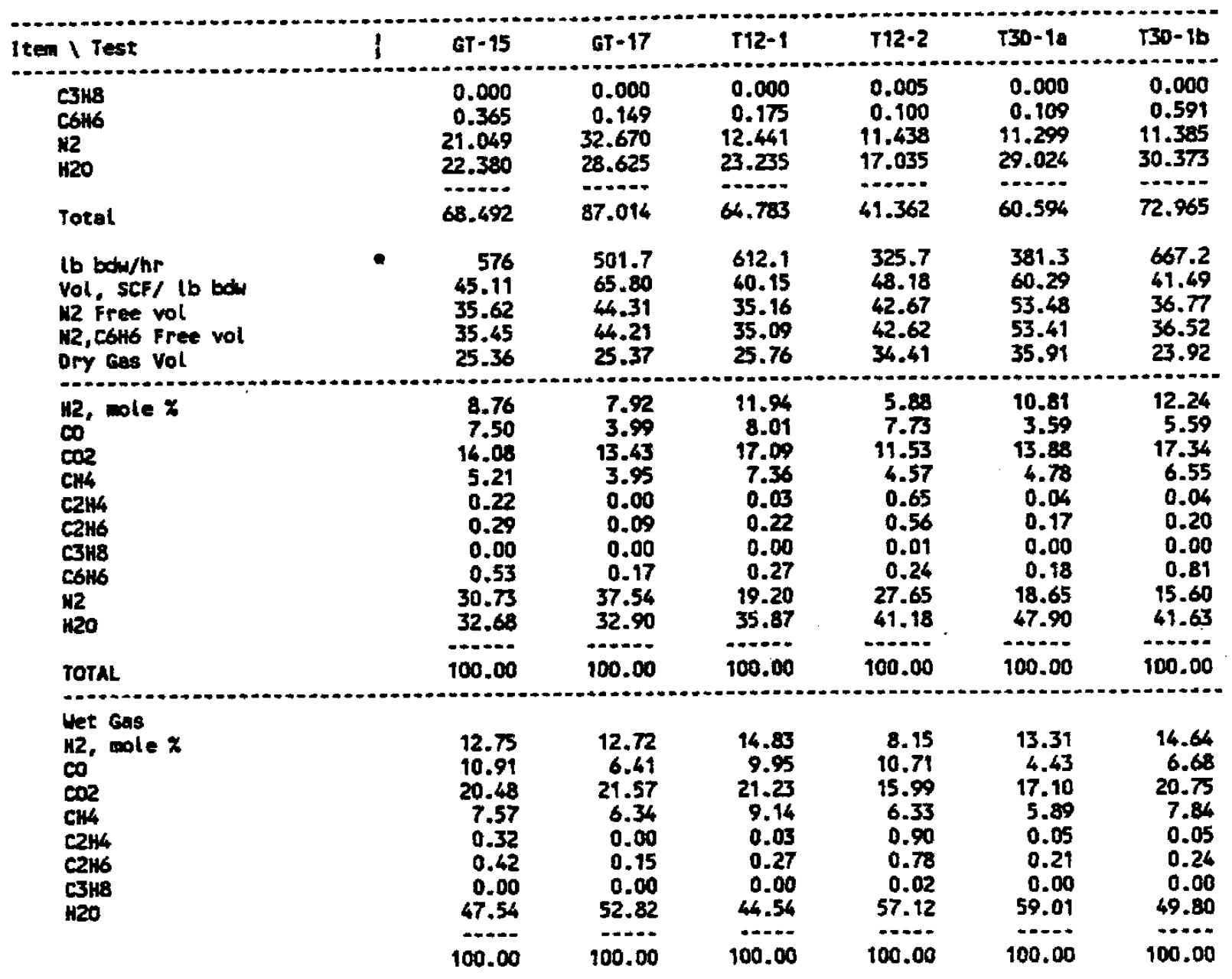




\begin{tabular}{|c|c|c|c|c|}
\hline Item I Test & $I 12-3 a$ & $T 12-3 b$ & $T 12-4 a$ & $T 12-4 b$ \\
\hline 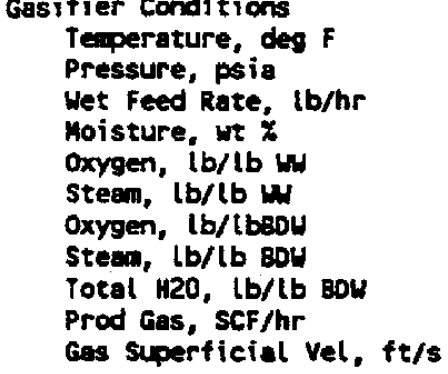 & $\begin{array}{r}1672 \\
317.7 \\
708.1 \\
9.14 \\
0.26 \\
0.69 \\
0.29 \\
0.76 \\
0.860 \\
27.472 \\
2.01\end{array}$ & $\begin{array}{r}1413 \\
317.7 \\
708.1 \\
9.14 \\
0.18 \\
0.68 \\
0.20 \\
0.75 \\
0.849 \\
25,373 \\
1.63\end{array}$ & $\begin{array}{r}1509 \\
83.7 \\
375.4 \\
8.98 \\
0.27 \\
0.72 \\
0.30 \\
0.79 \\
0.890 \\
16.813 \\
4.31\end{array}$ & $\begin{array}{r}1516 \\
98.7 \\
375.4 \\
9.59 \\
0.28 \\
0.73 \\
0.31 \\
0.81 \\
0.914 \\
17.439 \\
3.8\end{array}$ \\
\hline $\begin{array}{l}\text { Feed } \\
\text { Type } \\
\text { size }\end{array}$ & $\begin{array}{l}\text { Whe } \\
\text { Chips }\end{array}$ & $\begin{array}{l}\text { Hix } \\
\text { chips }\end{array}$ & chipe & $\begin{array}{c}\text { Wr } \\
\text { Chips }\end{array}$ \\
\hline $\begin{array}{l}\text { Proxinate Analysis } \\
\text { Moisture, wt X } \\
\text { Volatile Matter } \\
\text { Ash } \\
\text { Fixed Carbon (by diff) } \\
\text { Total }\end{array}$ & $\begin{array}{r}9.14 \\
75.89 \\
0.74 \\
16.24 \\
100.00\end{array}$ & $\begin{array}{r}9.14 \\
75.89 \\
0.74 \\
14.24 \\
100.00\end{array}$ & \begin{tabular}{r}
8.98 \\
76.02 \\
0.74 \\
14.26 \\
\hdashline$\ldots 0.0$ \\
100.00
\end{tabular} & $\begin{array}{r}9.59 \\
75.51 \\
0.73 \\
14.17 \\
100.00\end{array}$ \\
\hline $\begin{array}{l}\text { Ultiniete Anolysis } \\
\text { Ash, wt } x \text { dry } \\
\text { Carbon } \\
\text { Hydrogen } \\
\text { Sulfur } \\
\text { Nitrogen } \\
\text { Oxygen (by diff) }\end{array}$ & $\begin{array}{r}0.82 \\
48.40 \\
6.31 \\
0.03 \\
0.21 \\
44.23\end{array}$ & $\begin{array}{r}0.82 \\
48.40 \\
6.31 \\
0.03 \\
0.21 \\
44.23\end{array}$ & $\begin{array}{r}0.82 \\
48.40 \\
6.31 \\
0.03 \\
0.21 \\
44.23\end{array}$ & $\begin{array}{r}0.82 \\
48.40 \\
6.31 \\
0.03 \\
0.21 \\
44.23\end{array}$ \\
\hline $\begin{array}{l}\text { Total } \\
\text { HHV, BTU/Lb }\end{array}$ & $\begin{array}{r}100.00 \\
8389\end{array}$ & $\begin{array}{r}100.00 \\
8389\end{array}$ & $\begin{array}{r}100.00 \\
8389\end{array}$ & 8389 \\
\hline $\begin{array}{l}\text { Yield, Foreed elosure } \\
\text { Gas, we } x \text { of } C \\
\text { Tar/Oil, we } \mathrm{x} \text { of } \mathrm{C} \\
\text { Char, wt } \% \text { of } \mathrm{C} \\
\text { Tar/oil, wt } \mathrm{x} \text { maf feed }\end{array}$ & $\begin{array}{r}96.12 \\
2.16 \\
1.72 \\
1.13\end{array}$ & $\begin{array}{r}87.14 \\
6.72 \\
6.14 \\
3.54\end{array}$ & $\begin{array}{r}91.78 \\
5.12 \\
3.10 \\
2.73\end{array}$ & $\begin{array}{r}93.22 \\
4.74 \\
2.04 \\
2.51\end{array}$ \\
\hline
\end{tabular}




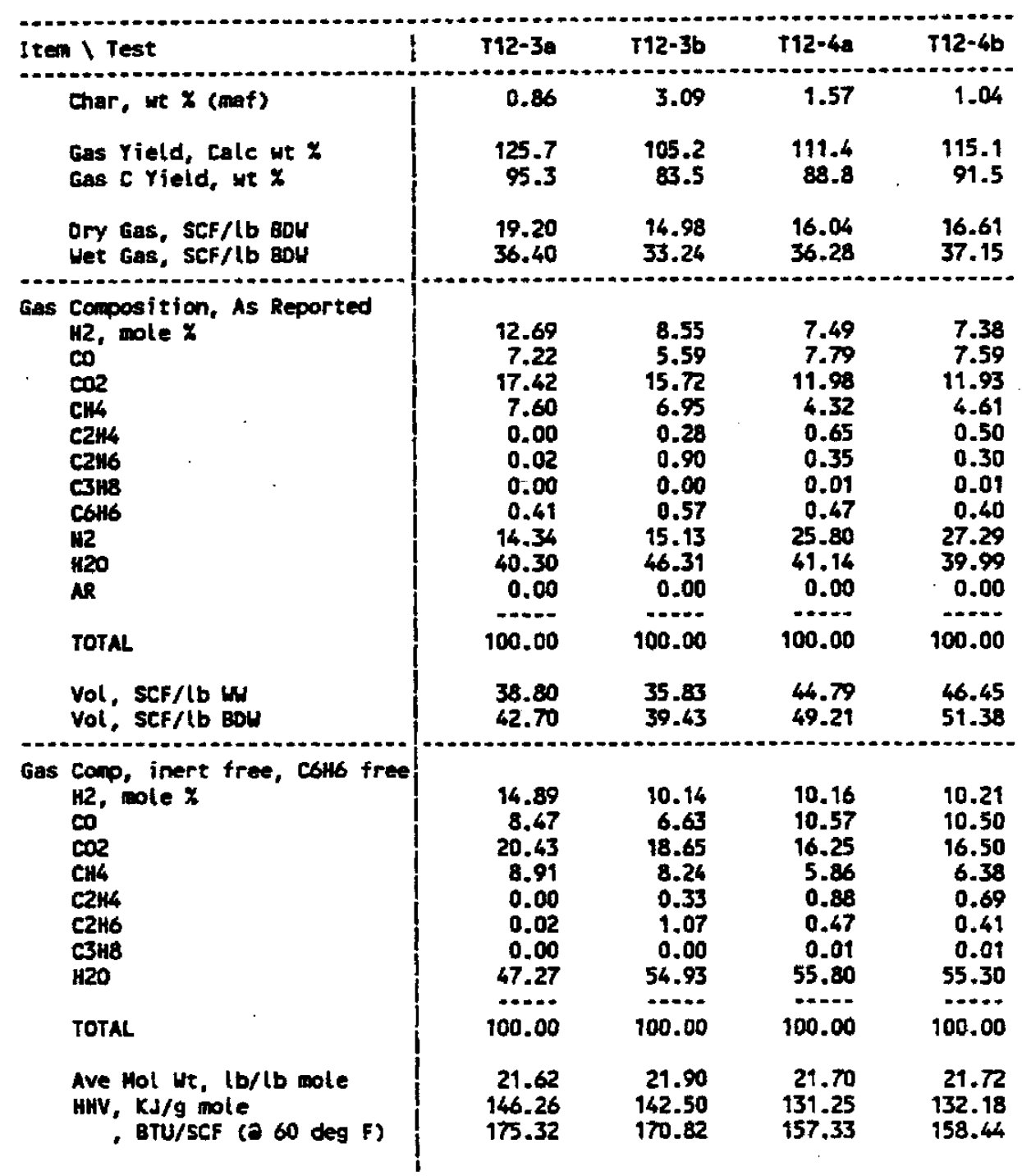




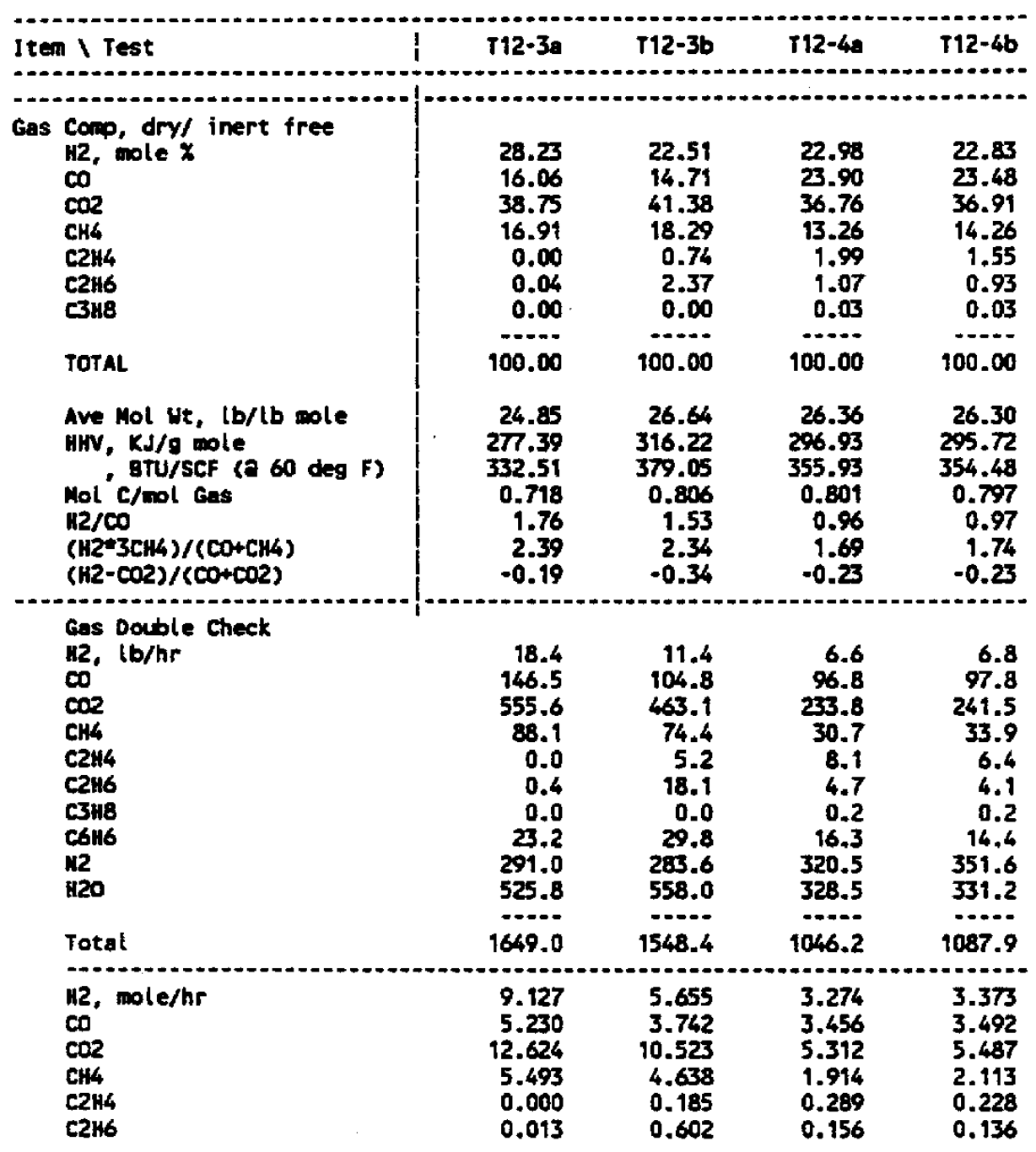




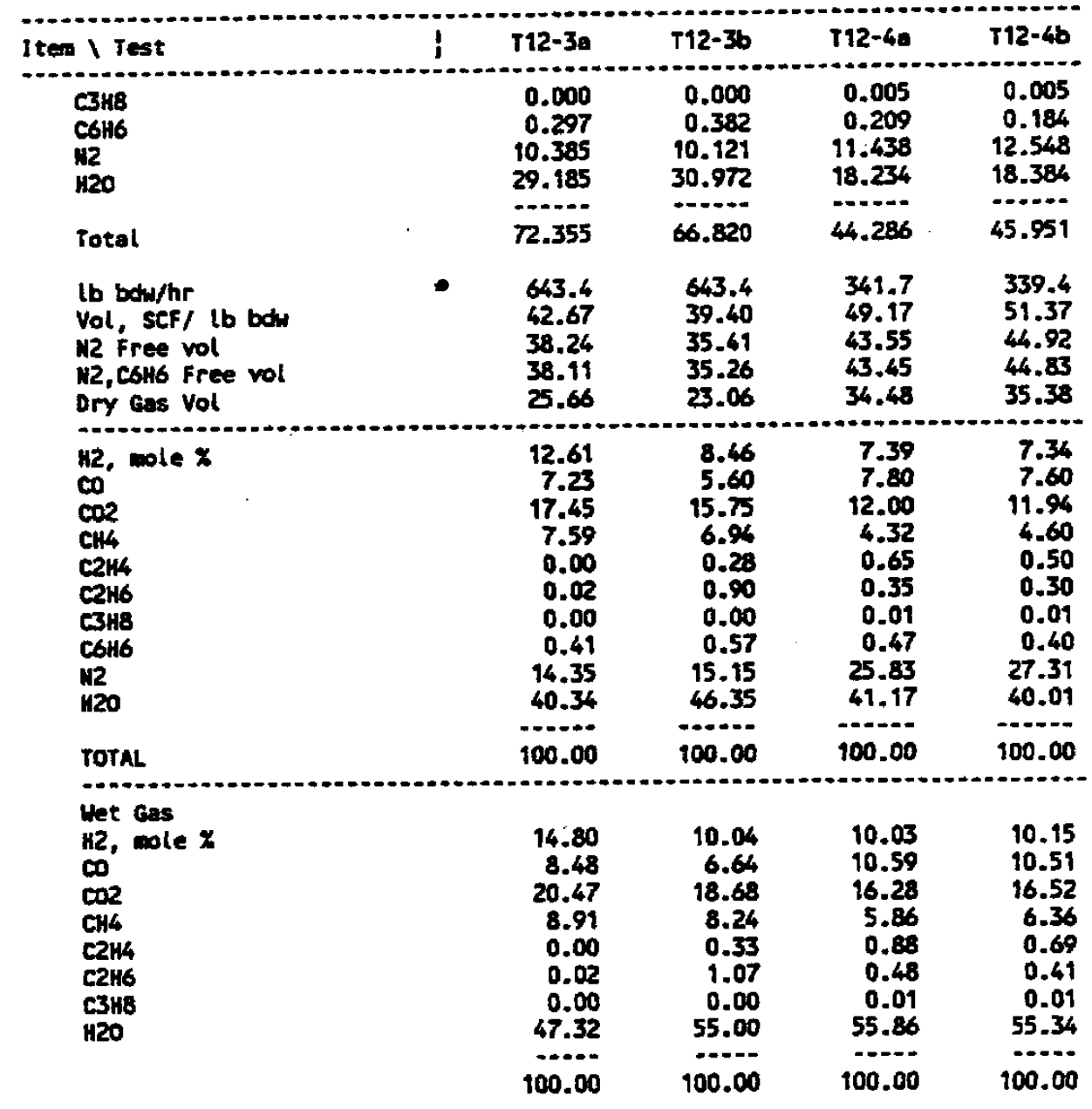


APPENDIX 2

53 
LEAST SOUARES FIT OF IGT GASIFIER VARIABLES

1. Hydrogen

Constant Regression Output:

Std Err of $Y$ Est

$-1.3528 E+01$

$R$ Squared

No. of Observations

$1.9229 E+00$

Degrees of Freedom

4.0984E-01

2.0000E+01

$1.7000 E+01$

$x$ Coefficient(s)

Std Err of Coef.

$1.7467 E-02-2.6444 E-07$

1.1374 E-01 3.5693E-05

t value

$0.153573727-0.00740873$

t Probability

0.1512628350 .007299769

0.439884279

0.497087863

f Value

5.902804634

0.111111111

0.013071895

2.278572097

Frobability

0.011346303

11. $\mathrm{CO}$

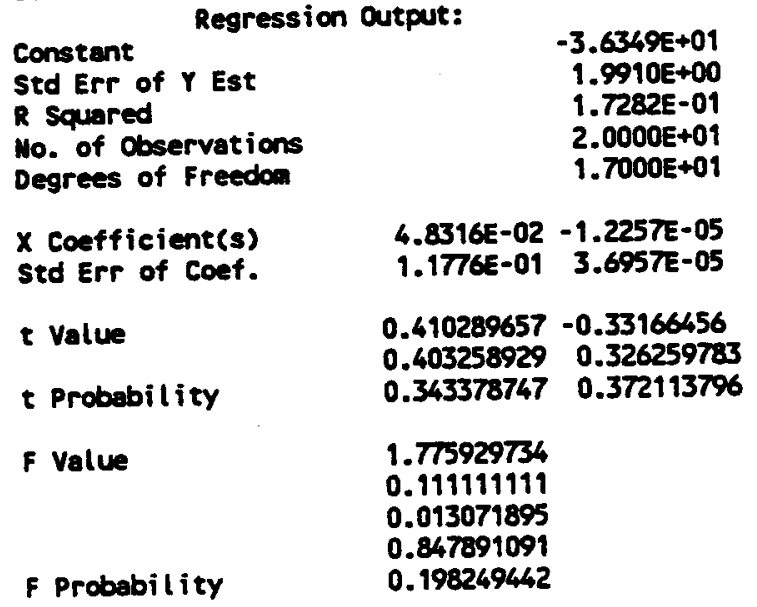

111. $\mathrm{CO2}$

\begin{tabular}{|c|c|c|}
\hline $\begin{array}{l}\text { Constant } \\
\text { Std Err of Y Est } \\
R \text { Squared } \\
\text { No. of Observations } \\
\text { Degrees of Freedom }\end{array}$ & tput: & $\begin{array}{r}-5.1963 E+01 \\
1.7287 E+00 \\
1.1919 E-01 \\
2.0000 E+01 \\
1.7000 E+01\end{array}$ \\
\hline $\begin{array}{l}x \text { Coefficient(s) } \\
\text { std Err of Coef. }\end{array}$ & $\begin{array}{l}8.4609 E-02 \\
1.0225 E-01\end{array}$ & $\begin{array}{r}-2.4793 E-05 \\
3.2089 E-05\end{array}$ \\
\hline $\begin{array}{l}\text { t Value } \\
\text { t Probability }\end{array}$ & $\begin{array}{l}0.827472113 \\
0.807215849 \\
0.209771196\end{array}$ & $\begin{array}{l}-0.7726484 \\
0.754689185 \\
0.225217863\end{array}$ \\
\hline Value & $\begin{array}{l}1.150177777 \\
0.111111111 \\
0.013071895 \\
0.409678427 \\
0.341020\end{array}$ & \\
\hline
\end{tabular}


IV. CH4

\begin{tabular}{|c|c|c|}
\hline \multicolumn{2}{|c|}{ Regression output: } & \\
\hline $\begin{array}{l}\text { Constant } \\
\text { Std Err of Y Est } \\
R \text { Squared } \\
\text { No. of Observations } \\
\text { Degrees of Freedom }\end{array}$ & & $\begin{array}{r}-4.3123 E+01 \\
1.1366 E+00 \\
5.0808 E-02 \\
2.0000 E+01 \\
1.7000 E+01\end{array}$ \\
\hline $\begin{array}{l}x \text { Coefficient(s) } \\
\text { std Err of Coef. }\end{array}$ & $\begin{array}{l}6.4007 E-02 \\
6.7229 E-02\end{array}$ & $\begin{array}{r}-2.0016 E-05 \\
2.1098 E-05\end{array}$ \\
\hline $\begin{array}{l}\text { t Value } \\
\text { t Probability }\end{array}$ & $\begin{array}{l}0.952071729 \\
0.925810784 \\
0.177272269\end{array}$ & $\begin{array}{c}-0.94868564 \\
0.922603153 \\
0.178107135\end{array}$ \\
\hline Probability & $\begin{array}{c}0.454980481 \\
0.111111111 \\
0.013071895 \\
-0.37599196 \\
0.646529332\end{array}$ & \\
\hline
\end{tabular}

v. $\mathrm{C2H4}$

$\begin{array}{ll}\text { Constant Regression Output: } \\ \text { Std Err of } Y \text { Est } \\ \text { R Squared } \\ \text { Mo. of Observations } \\ \text { Degrees of Freedom } \\ \text { X Coefficient(s) } & -1.1442 E-03 \\ \text { Std Err of Coef. } & 7.1612 E-04 \\ & \\ t \text { Value } & -1.59778852 \\ & 1.522536506 \\ t \text { Probability } & 0.063937262 \\ & \\ \text { F Value } & 2.552928155 \\ & 0.222222222 \\ & 0.012345679 \\ & 1.154218815 \\ \text { F Probability } & 0.124205188\end{array}$

v1. c2116

Constant

Regression output:

Sed Err of $Y$ Est

$2.0095 E+00$

$2.8460 \mathrm{E}-01$

1.2421E-01

2.0000E+01

$1.8000 E+01$

$R$ Squared

No. of Observations

Degrees of Freedon

$2.6517 E+01$

$1.3753 \mathrm{E}-01$

8.1572E-01

$2.0000 E+01$

$1.7000 E+01$

$x$ Coefficient(s)

$-3.0323 E-02 \quad 8.6568 E-06$

Std Err of Coef.

8.1347E-03 2.5529E-06

t Value

$-3.727559653 .390956626$

t Probability

3.094469582 .888209082

F Value

0.000985775

0.001937095

F Probability

37.62440422

0.111111111

0.013071895

4.75231605

1.0337E-06 


$\begin{array}{ll}\text { V11. C3H8 } & \\ & \\ \text { Constant Regression Output: } & 4.9248 E-02 \\ \text { Std Err of Y Est } & 6.8449 E-03 \\ \text { R Squared } & 1.4156 E-01 \\ \text { No. of Observations } & 2.0000 E+01 \\ \text { Degrees of Freedom } & 1.8000 E+01 \\ & \\ \text { X Coefficient(s) } & -2.9673 E-05 \\ \text { Std Err of Coef. } & 1.7223 E-05 \\ & \\ \text { t Value } & -1.72285127 \\ & 1.632937739 \\ \text { t Probability } & 0.051241018\end{array}$

v111. 120

$$
\text { Regression output: }
$$

Constant

Std Err of $Y$ Est

R squared

No. of Observations

Degrees of Freedom

2.2154E+02

$3.8295 E+00$

3.4492E-01

2.0000E+01

$X$ Coefficient( $s$ )

$1.7000 E+01$

std Err of Coef.

$-1.8940 E-01$ 5.0717E-05

2.2650E-01 7.1083E-05

r Value

$\begin{array}{lll}-0.83621181 & 0.713482906\end{array}$

Probability

0.815570670 .69778619

f value

0.2073729950 .242655542

Falue

4.459693009

0.1111111111

0.013071895

F Probability

1.919489163

0.027461344

IX. Tar

\begin{tabular}{|c|c|c|}
\hline $\begin{array}{l}\text { Constant } \\
\text { Std Err of Y Est } \\
\text { R Squared } \\
\text { No. of Observations } \\
\text { Degrees of Freedom }\end{array}$ & pe & $\begin{array}{l}2.2739 E+01 \\
6.7970 E-01 \\
4.3102 E-01 \\
2.0000 E+01 \\
1.7000 E+01\end{array}$ \\
\hline $\begin{array}{l}x \text { coefficient(s) } \\
\text { Std Err of Coef. }\end{array}$ & $\begin{array}{r}-2.0584 E-02 \\
4.0202 E-02\end{array}$ & $\begin{array}{l}4.5476 E-06 \\
1.2617 E-05\end{array}$ \\
\hline $\begin{array}{l}\text { Value } \\
\text { Probability }\end{array}$ & $\begin{array}{c}-0.51201629 \\
0.502552864 \\
0.307639255\end{array}$ & $\begin{array}{l}0.360446762 \\
0.354469465 \\
0.361493418\end{array}$ \\
\hline F Volue & $\begin{array}{l}6.439100545 \\
0.1111111111 \\
0.013071895 \\
2.392352073 \\
0.008370372\end{array}$ & \\
\hline
\end{tabular}


X. Char

$\begin{array}{ll}\text { Char } & \\ & \\ \text { Constant Regression Output: } & 1.2722 E+01 \\ \text { Std Err of Y Est } & 1.2802 E+00 \\ \text { R Squared } & 1.7921 E-01 \\ \text { Mo. of Observations } & 2.0000 E+01 \\ \text { Degrees of Freedom } & 1.8000 E+01 \\ & \\ \text { X Coefficient(s) } & -6.3858 E-03 \\ \text { Std Err of Coef. } & 3.2212 E-03 \\ & \\ \text { t Value } & -1.98241538 \\ & 1.856189302 \\ \text { t Probability } & 0.03171339\end{array}$

XI. Dry Gas

Constant

Regression output:

Std Err of Y Est

$R$ Squared

Mo. of Observations

Degrees of Freedom

$0.0000 E+00$

$4.8097 \mathrm{E}+00$

$9.0780 E-03$

2.0000E+01

$X$ Coefficient(s)

$1.8000 E+01$

std Err of Coef.

3.0627E-02 -8.8818E-06

$1.1270 E-02 \quad 7.3156 E-06$

t Value

$2.717485525-1.21409508$

t Probability

$2.44104567 \quad 1.173450083$

Probability

0.00732236

0.120307621

F Value

0.164900645

0.222222222

0.012345679

$-0.49619202$

F Probability

0.690104404

XII. Het Gas

Constant

Std Err of Y Est

$R$ squared

No. of Observations

Degrees of Freedom

$1.8000 E+01$

$X$ Coefficient(s)

4.5549E-02 - $1.3377 E-05$

std Err of Coef.

9.1773E-03 5.9570E-06

$t$ Value

$4.963198082-2.24559468$

t Probability

3.771229671

2.073913012

F Value

0.132778899

0.222222222

0.012345679

F Probability

$-0.57618809$ 
APPENDIX 3

$\underline{58}$ 
IGT Gasifier - Conditions and Correlations

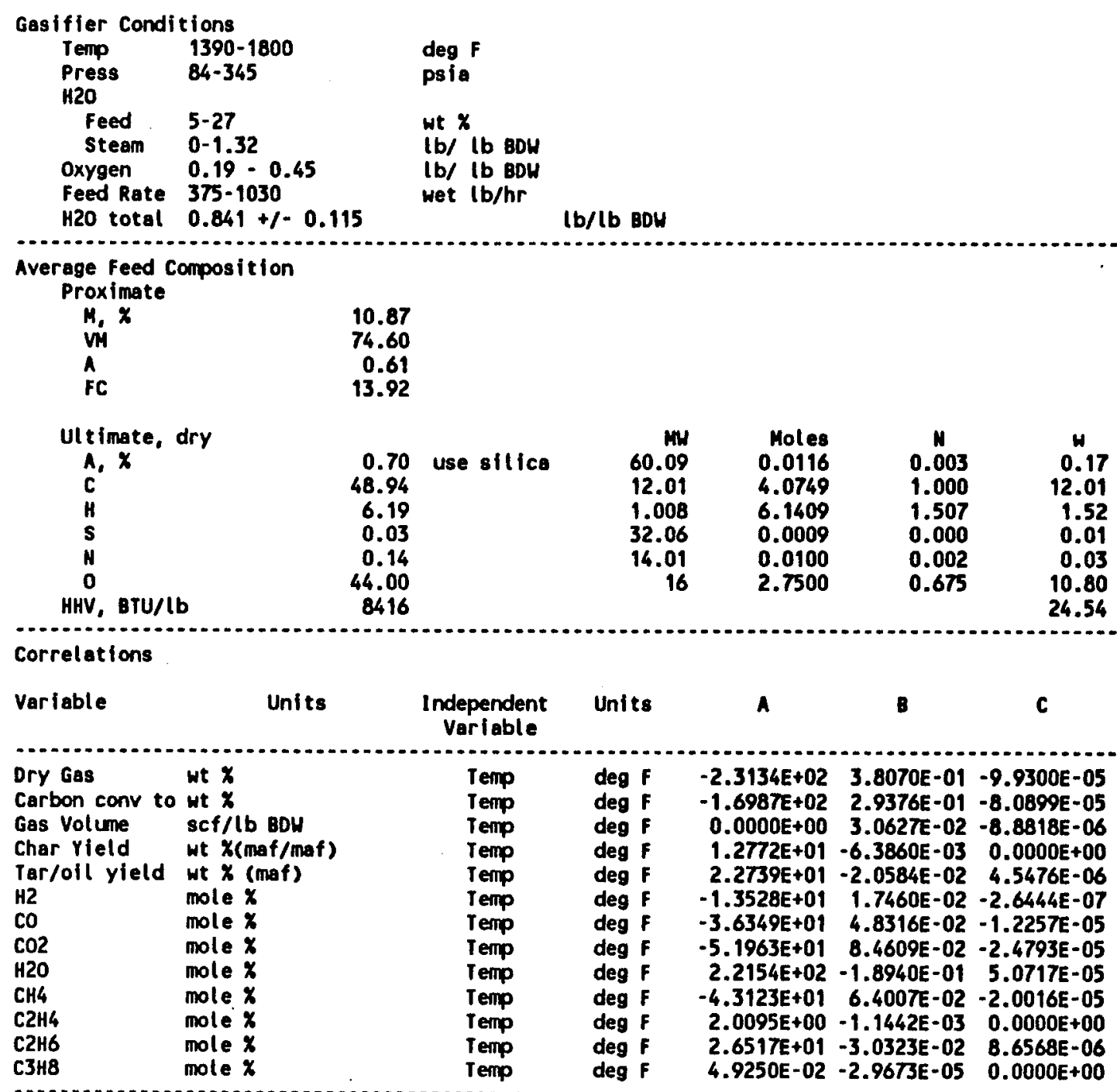


APpenOTX 4 
ASPEN INPUT DATA - IGT GASIFIER

Temperature

$\begin{array}{rr}\text { Steam } & \mathrm{lb} / \mathrm{hr} \\ \text { Oxygen } & \mathrm{lb} / \mathrm{hr} \\ \mathrm{H} & \mathrm{lb} / \mathrm{hr} \\ \mathrm{CO} & \mathrm{lb} / \mathrm{hr} \\ \mathrm{CH4} & \mathrm{lb} / \mathrm{hr} \\ \mathrm{C2H4} & \mathrm{lb} / \mathrm{hr} \\ \mathrm{C2H6} & \mathrm{lb} / \mathrm{hr} \\ \mathrm{C3H8} & \mathrm{lb} / \mathrm{hr} \\ \mathrm{CO2} & \mathrm{lb} / \mathrm{hr} \\ \mathrm{H} 2 \mathrm{O} & \mathrm{lb} / \mathrm{hr} \\ \text { Char } & \mathrm{lb} / \mathrm{hr} \\ \text { Benzene } & \mathrm{lb} / \mathrm{hr} \\ \text { Naphthalene } & \mathrm{lb} / \mathrm{hr} \\ \text { Anthracene } & \mathrm{lb} / \mathrm{hr} \\ \text { O-cresol } & \mathrm{lb} / \mathrm{hr} \\ & \\ \text { In } & \mathrm{lb} / \mathrm{hr} \\ \text { Out } & \mathrm{lb} / \mathrm{hr} \\ \text { Diff } & \mathrm{lb} / \mathrm{hr}\end{array}$

Steam

as steam $\quad \mathrm{lb} / \mathrm{hr}$

os moisture

lb/hr

b/hr

b/hr
MH

1400

1450

1500

1550

1600

1650

1700

1750

1800

$18.016140,166.667140,166.667 \quad 140,166.667140,166.667 \quad 140,166.667140,166.667 \quad 140,166.667 \quad 140,166.667140,166.667$

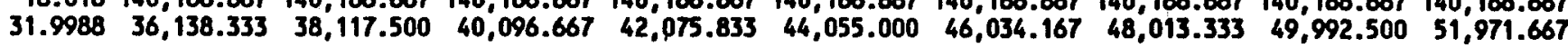

$2.0158 \quad 3,195.131 \quad 3,468.716 \quad 3,750.140$

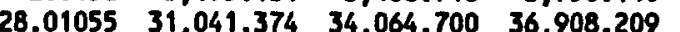

$28.01055 \quad 31,041.374 \quad 34,064.700 \quad 36,908.209$

$\begin{array}{lll}16.04275 & 17,743.797 & 18,686.065\end{array}$

$\begin{array}{lll}28.0539 & 1,743.222 & 1,505.895 \\ 30.0697 & 4.731 .149 & 3.452 .76\end{array}$

$19,419.220$

$4,340.918 \quad 4,653.034$

$44,415.580$ 46, 591.764

$5,318.8815,678.002$

$44.09665 \quad 51.813 \quad 3.42 .045$

$2,365.675$

$1,028.705$

246.813

$0,332.578$

$48,613.809 \quad 50,492.668$

$\begin{array}{rrrrrrrrr}4.731 .149 & 3,452.768 & 2,365.675 & 1,468.567 & 762.119 & 248.769 & 0.000 & 0.000 & 0.000 \\ 51.813 & 42.045 & 32.228 & 22.316 & 12.263 & 2.024 & 0.000 & 0.000 & 0.000\end{array}$

468.567

786.580

540.649

190.205

289.740

$19,812.22$

$\begin{array}{rrrrrrrrr}4.731 .149 & 3,452.768 & 2,365.675 & 1,468.567 & 762.119 & 248.769 & 0.000 & 0.000 & 0.000 \\ 51.813 & 42.045 & 32.228 & 22.316 & 12.263 & 2.024 & 0.000 & 0.000 & 0.000\end{array}$

32.636

$\begin{array}{rrrrrrrrr}4.731 .149 & 3,452.768 & 2,365.675 & 1,468.567 & 762.119 & 248.769 & 0.000 & 0.000 & 0.000 \\ 51.813 & 42.045 & 32.228 & 22.316 & 12.263 & 2.024 & 0.000 & 0.000 & 0.000\end{array}$

0.000

120,058.896 125,348.700 130,043.414 134,140.526 137,637.150 140,529.900 142,804.512 144,459.194 145,499.570

$18.016153 .217 .469148,368.805144,267.373140,913.981 \quad 138,309,090136,452.887135,284.957134,828.561135,092.552$

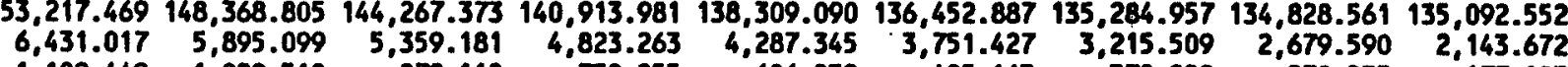

$78.1143 \quad 1,189.449 \quad 1,029.510$

$128.1143 \quad 1,189.449 \quad 1,029.510$

$178.2351 \quad 1,189.449 \quad 1,029.510$

879.112

738.255

606.939

485.163

372.929

270.235

177.083

$1,189.449 \quad 1,029.510$

738.112

$879.112 \quad 738.255$

606.939

485.163

485.163

485.163

372.929

270.235

177.083

$342,971.667344,950.833 \quad 346,930.000348,909.167350,888.333 \quad 352,867.500354,846.667356,825.833358,805.000$ $342,971.667344,950.833346,930.000348,909.167350,884$ (0.000)

0.000

$(0.000)$

0.000

0.000

(0.000)

0.000

0.000

0.000

$\begin{array}{lll}119840.55 & 119840.55 & 119840.55\end{array}$

$20326.12 \quad 20326.12 \quad 20326.12$ 
$\begin{array}{ll}\text { oxygen } & \mathrm{lb} / \mathrm{hr} \\ \text { steam } & \mathrm{tb} / \mathrm{hr}\end{array}$

Steam lb/hr
$10.87 \%$ M Feedlb/hr

Total lb/hr

Yield

\section{H2 lb/lb feed}

co

CH4

$\mathrm{C2H4}$

C2H6

$\mathrm{CO} 2$

Char

Benzene

Naphthalene

Anthracene

o-cresol

$$
\text { Total }
$$

for Wwood at $50 \times \mathrm{M}$

Total H2O

H2O

as $H$

HHood

\begin{tabular}{|c|c|c|c|c|c|c|c|c|}
\hline 1400 & 1450 & 1500 & 1550 & 1600 & 1650 & 1700 & 1750 & 1800 \\
\hline $\begin{array}{r}1129.365 \\
6651.896 \\
186992.782\end{array}$ & $\begin{array}{r}1191.217 \\
6651.896 \\
186992.782\end{array}$ & $\begin{array}{r}1253.068 \\
6651.896 \\
186992.782\end{array}$ & $\begin{array}{r}1314.919 \\
6651.896 \\
186992.782\end{array}$ & $\begin{array}{r}1376.770 \\
6651.896 \\
186992.782\end{array}$ & $\begin{array}{r}1438.622 \\
6651.896 \\
186992.782\end{array}$ & $\begin{array}{r}1500.473 \\
6651.896 \\
186992.782\end{array}$ & $\begin{array}{r}1562.324 \\
6651.896 \\
186992.782\end{array}$ & $\begin{array}{r}1624.175 \\
6651.896 \\
186992.782\end{array}$ \\
\hline $\begin{array}{r}36139.689 \\
119840.551 \\
186992.782\end{array}$ & $\begin{array}{r}38118.929 \\
119840.551 \\
186992.782\end{array}$ & $\begin{array}{r}40098.170 \\
119840.551 \\
186992.782\end{array}$ & $\begin{array}{r}42077.411 \\
119840.551 \\
186992.782\end{array}$ & $\begin{array}{r}44056.652 \\
119840.551 \\
186992.782\end{array}$ & $\begin{array}{r}46035.893 \\
119840.551 \\
186992.782\end{array}$ & $\begin{array}{r}48015.134 \\
119840.551 \\
186992.782\end{array}$ & $\begin{array}{r}49994.375 \\
119840.551 \\
186992.782\end{array}$ & $\begin{array}{r}51973.616 \\
119840.551 \\
186992.782\end{array}$ \\
\hline 342973.022 & 344952.263 & 346931.504 & 348910.745 & 350889.985 & 352869.226 & 354848.467 & 356827.708 & 358806.949 \\
\hline $\begin{array}{l}9.3160 E-03 \\
9.0507 E-02 \\
5.1735 E-02 \\
5.0827 E-03 \\
1.3795 E-02 \\
1.5107 E-04 \\
3.5005 E-01 \\
4.4673 E-01 \\
1.8751 E-02 \\
3.4681 E-03 \\
3.4681 E-03 \\
3.4681 E-03 \\
3.4681 E-03 \\
1.0000 E+00\end{array}$ & $\begin{array}{l}1.0056 E-02 \\
9.8752 E-02 \\
5.4170 E-02 \\
4.3655 E-03 \\
1.0009 E-02 \\
1.2189 E-04 \\
3.6338 E-01 \\
4.3011 E-01 \\
1.7090 E-02 \\
2.9845 E-03 \\
2.9845 E-03 \\
2.9845 E-03 \\
2.9845 E-03 \\
1.0000 E+00\end{array}$ & $\begin{array}{l}1.0809 E-02 \\
1.0638 E-01 \\
5.5974 E-02 \\
3.6552 E-03 \\
6.8189 E-03 \\
9.2894 E-05 \\
3.7484 E-01 \\
4.1584 E-01 \\
1.5447 E-02 \\
2.5340 E-03 \\
2.5340 E-03 \\
2.5340 E-03 \\
2.5340 E-03 \\
1.0000 E+00\end{array}$ & $\begin{array}{l}1.1580 E-02 \\
1.1343 E-01 \\
5.7151 E-02 \\
2.9483 E-03 \\
4.2090 E-03 \\
6.3958 E-05 \\
3.8446 E-01 \\
4.0387 E-01 \\
1.3824 E-02 \\
2.1159 E-03 \\
2.1159 E-03 \\
2.1159 E-03 \\
2.1159 E-03 \\
1.0000 E+00\end{array}$ & $\begin{array}{l}1.2371 E-02 \\
1.1992 E-01 \\
5.7701 E-02 \\
2.2417 E-03 \\
2.1720 E-03 \\
3.4948 E-05 \\
3.9225 E-01 \\
3.9417 E-01 \\
1.2218 E-02 \\
1.7297 E-03 \\
1.7297 E-03 \\
1.7297 E-03 \\
1.7297 E-03 \\
1.0000 E+00\end{array}$ & $\begin{array}{l}1.3186 E-02 \\
1.2587 E-01 \\
5.7621 E-02 \\
1.5322 E-03 \\
7.0499 E-04 \\
5.7360 E-06 \\
3.9825 E-01 \\
3.8670 E-01 \\
1.0631 E-02 \\
1.3749 E-03 \\
1.3749 E-03 \\
1.3749 E-03 \\
1.3749 E-03 \\
1.0000 E+00\end{array}$ & $\begin{array}{l}1.4029 E-02 \\
1.3130 E-01 \\
5.6898 E-02 \\
8.1652 E-04 \\
0.0000 E+00 \\
0.0000 E+00 \\
4.0244 E-01 \\
3.8125 E-01 \\
9.0616 E-03 \\
1.0510 E-03 \\
1.0510 E-03 \\
1.0510 E-03 \\
1.0510 E-03 \\
9.9999 E-01\end{array}$ & $\begin{array}{l}1.4906 E-02 \\
1.3624 E-01 \\
5.5523 E-02 \\
9.1462 E-05 \\
0.0000 E+00 \\
0.0000 E+00 \\
4.0484 E-01 \\
3.7785 E-01 \\
7.5095 E-03 \\
7.5733 E-04 \\
7.5733 E-04 \\
7.5733 E-04 \\
7.5733 E-04 \\
9.9999 E-01\end{array}$ & $\begin{array}{l}1.5825 E-02 \\
1.4072 E-01 \\
5.3483 E-02 \\
0.0000 E+00 \\
0.0000 E+00 \\
0.0000 E+00 \\
4.0551 E-01 \\
3.7650 E-01 \\
5.9744 E-03 \\
4.9353 E-04 \\
4.9353 E-04 \\
4.9353 E-04 \\
4.9353 E-04 \\
9.9999 E-01\end{array}$ \\
\hline
\end{tabular}

166666.667

146340.551

20326.115

8122.810 
APPENDIX 5 
TITLE 'GASIFIER - REFORMER - METHANOL SYNTHESIS - HNEI CASE A' ;GASIFIER - RYIELD MODEL

;REFORMER - EQUILIBRIUM MODEL. METHANOL - EQUILIBRIUM MODEL

;UPDATED: DECEMBER 5, 1991

;

IN-UNITS ENG

OUT-UNITS SI TEMPERATURE $=C$ PRESSURE $=$ BAR

HISTORY MSG-LEVEL PROPERTIES $=4$ SIMULATION=4 STREAMS $=4$ SYSTEM=4 ; $\begin{array}{ll}\text { RUN-CONTROL } & \text { MAX-TIME }=600 \\ \text { SIM-OPTIONS } & \text { SIZE-RESULTS }=0\end{array}$

;

STREAM-REPORT

STREAMS ALL

FLOW-FRAC MIXED BASES $=$ MOLE MOLE-FRAC

INTENSIVE-PROPS MIXED PROPS=TEMP PRES MW ENTH DENS BASE =MOLE

FLOW-FRAC CISOLID BASES = MASS

INTENSIVE-PROPS CISOLID PROPS=TEMP PRES ENTH DENS BASE=MASS

FLOW-FRAC NCPSD BASES = MASS

INTENSIVE-PROPS NCPSD PROPS=TEMP PRES ENTH DENS BASE=MASS

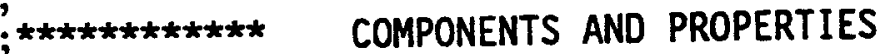
PROPERTIES SYSOPO / SYSOP3R LOOP2

; COMPONENTS

H2

CO

$\mathrm{CO} 2$

$\mathrm{H} 2 \mathrm{O}$

$\mathrm{CH} 4$

$\mathrm{C} 2 \mathrm{H} 2$

$\mathrm{C} 2 \mathrm{H} 4$

$\mathrm{C} 2 \mathrm{H} 6$

C 3 H6-2

C3H8

$\mathrm{MEOH}$

02

N2

AR

C6H6

C6H6O

$\mathrm{C} 1 \mathrm{OH} 8$

C14H10-1

C7H80-4

C

02SI

CHAR

WOOD

;

ALIAS

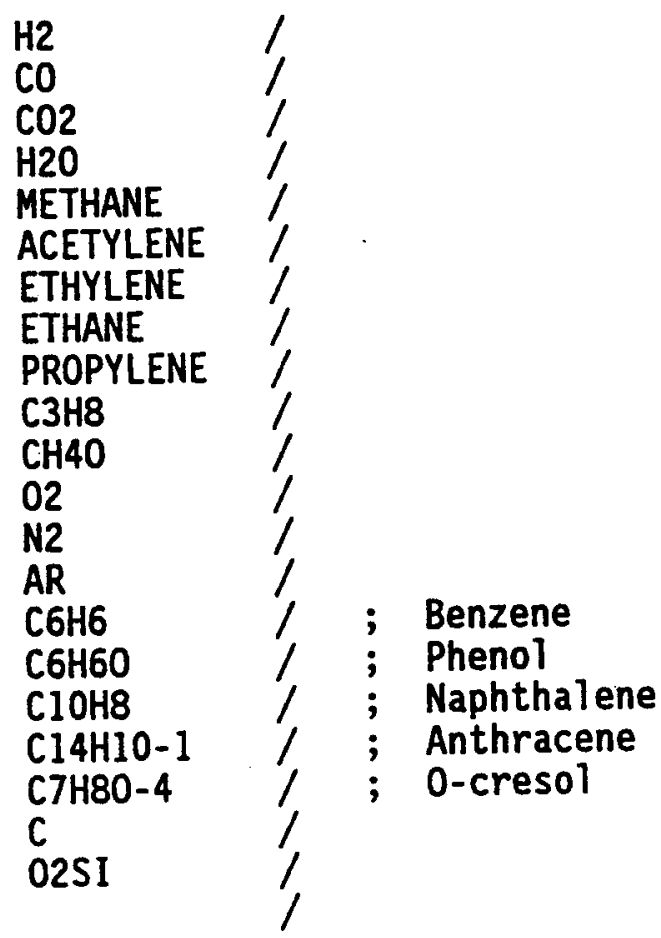




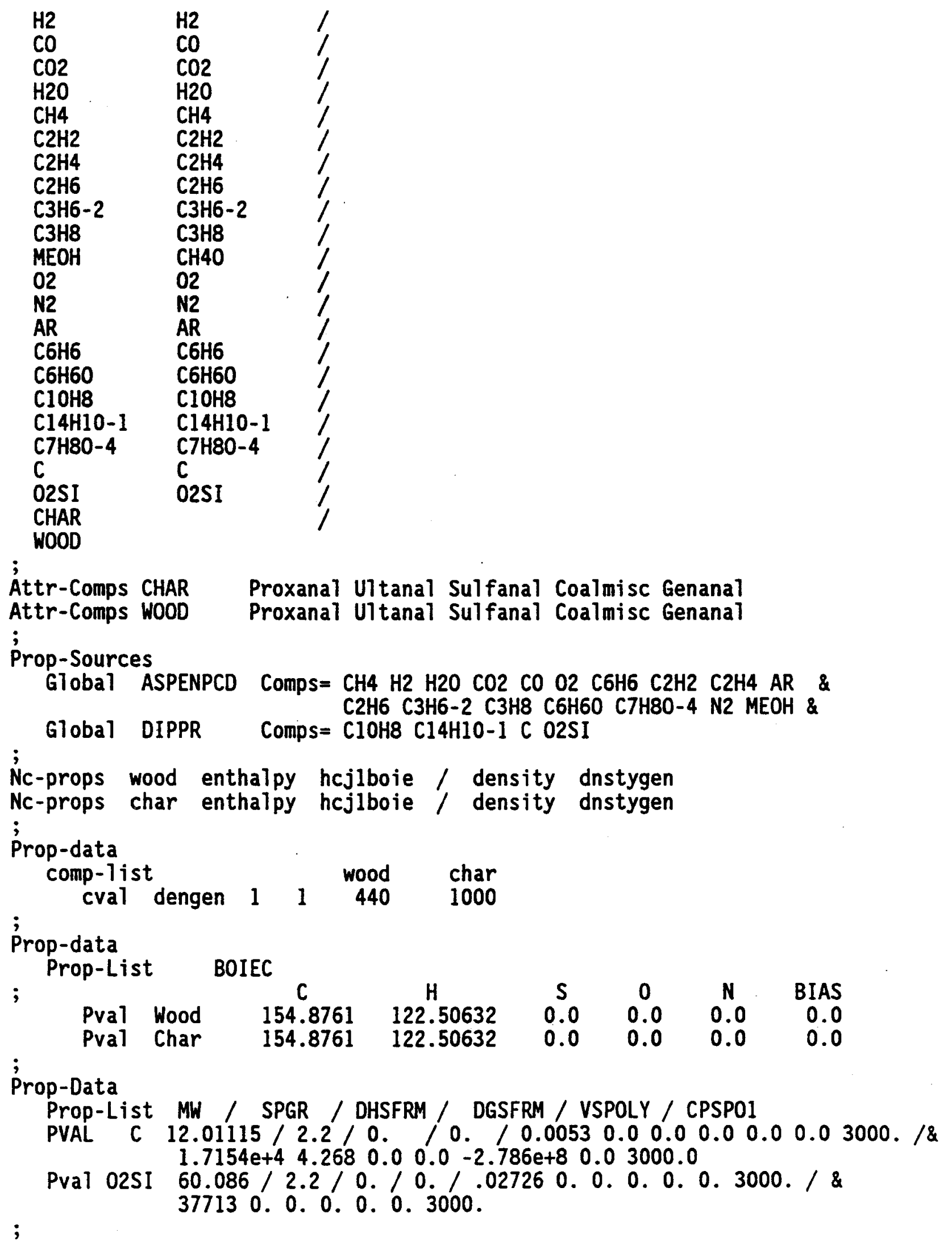




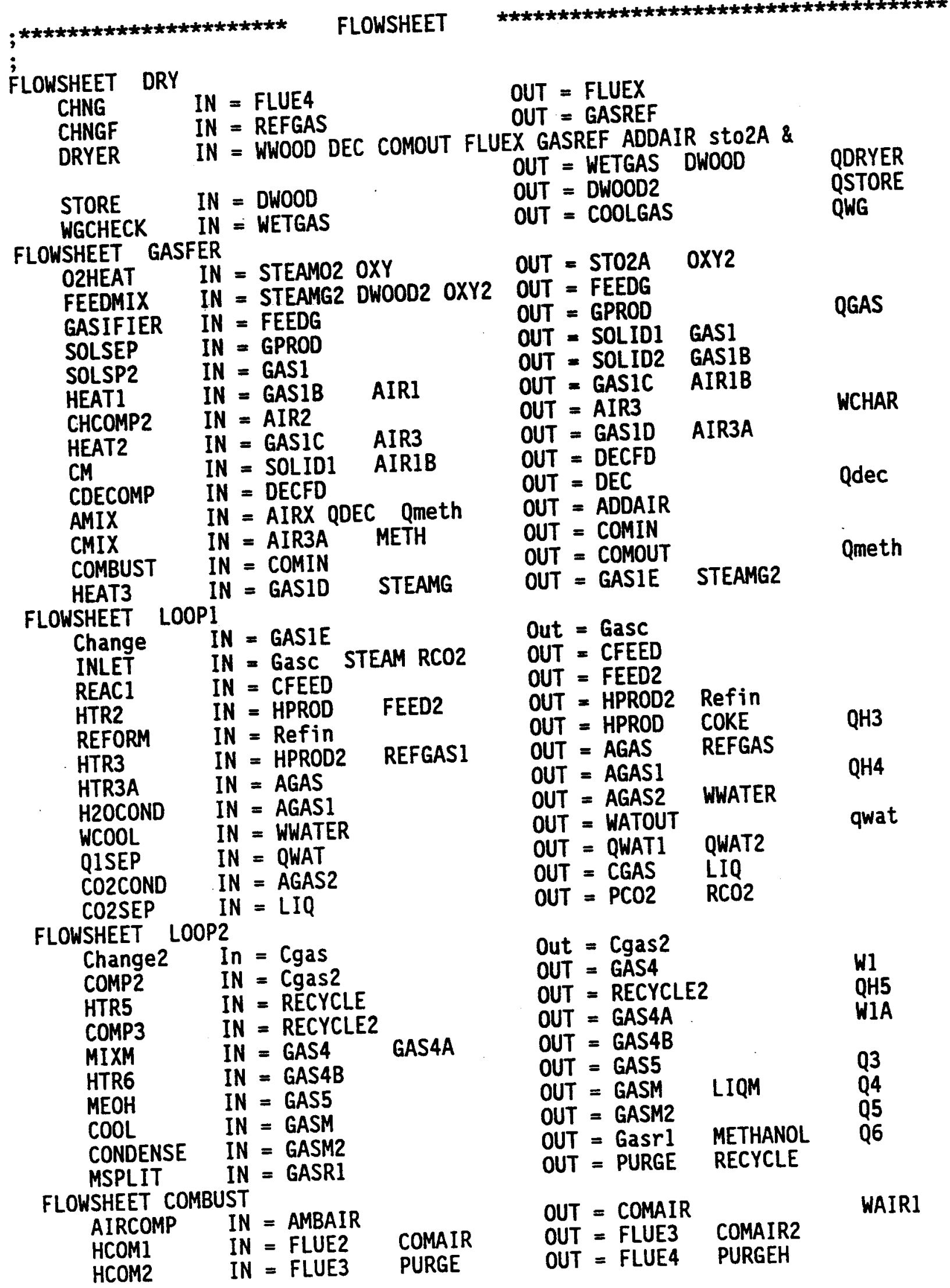




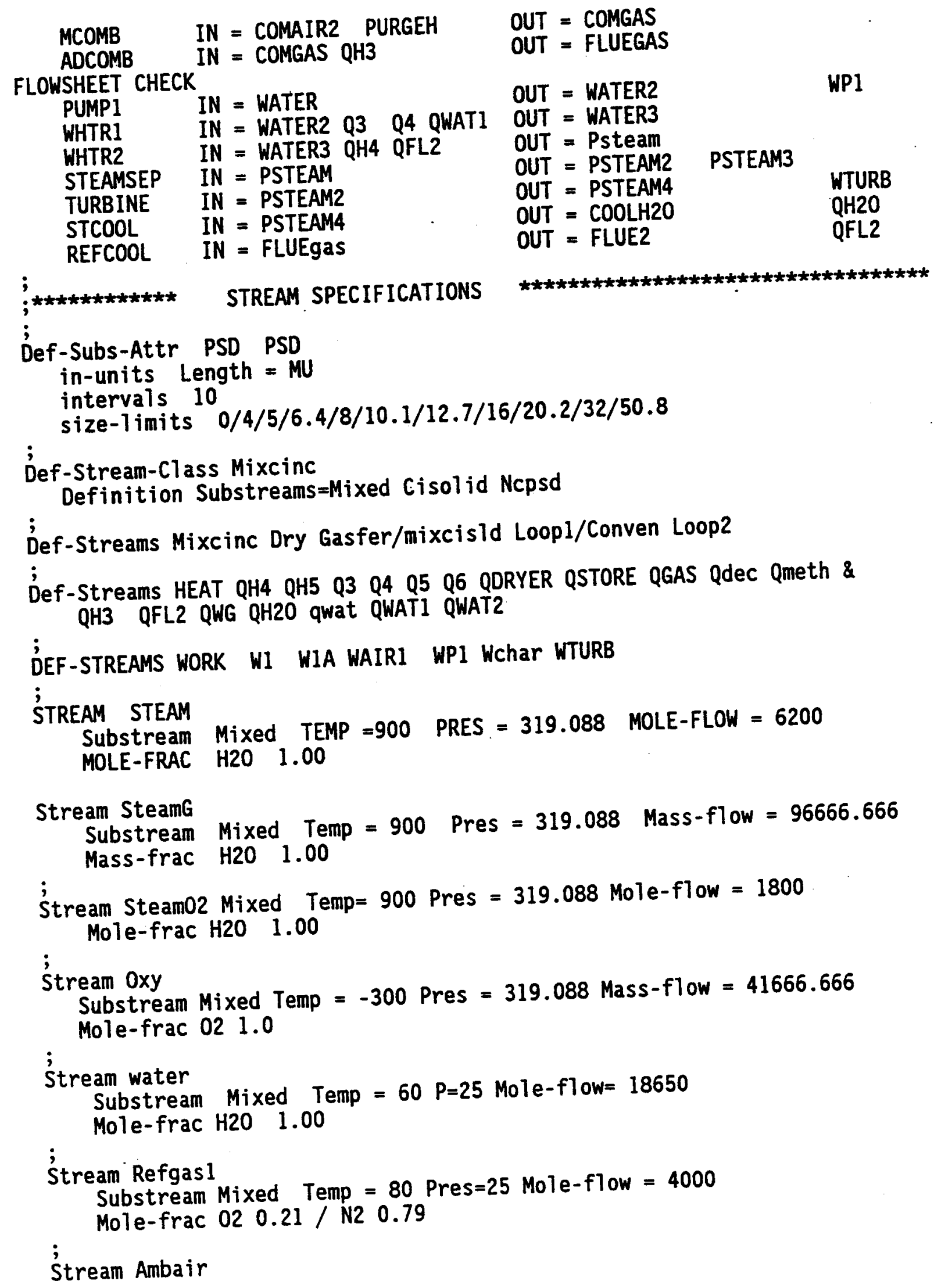




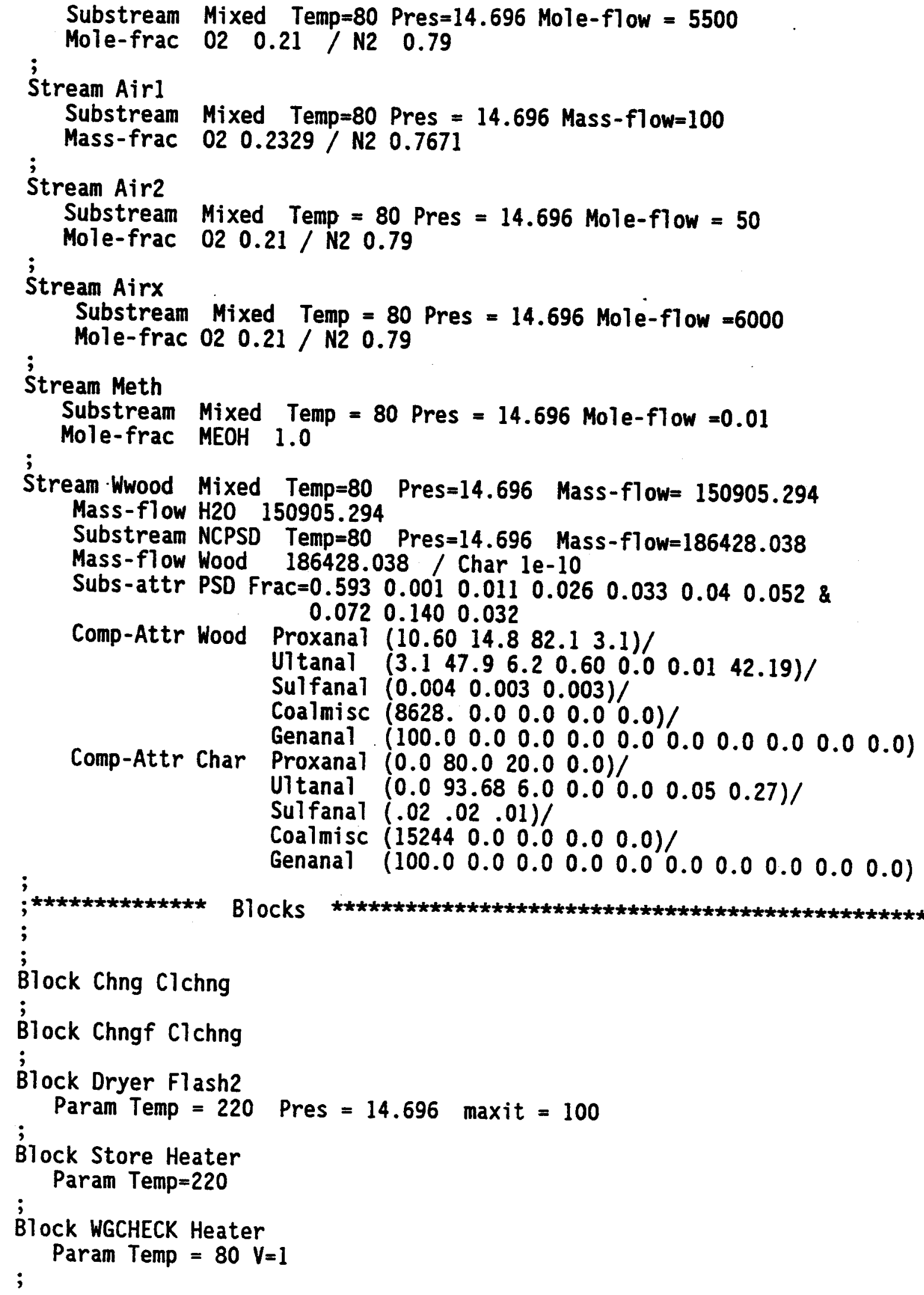


Block 02Heat Heatx

Param 0pt $=2$ Flowdir $=0$ Tcold $=600$

;

Block Feedmix Mixer

Param Pres $=319.088$

;

Block Gasifier Ryield

Properties Option=SYSOPO

Param Temp $=1526$ Pres $=319.088$

Massyield Ssid $=$ Mixed

Ssid $=$ Mixed

Ssid $=$ Mixed

Ssid $=$ Mixed

Ssid $=$ Mixed

Ssid $=$ Mixed

Ssid $=$ Mixed

Ssid $=$ Mixed

Ssid $=$ Mixed

Ssid $=$ Mixed

Ssid $=$ Mixed

Ssid $=$ Mixed

Comp $=\mathrm{H} 2$

$\mathrm{Val}=1.51109 \mathrm{E}-02 /$

Comp $=\mathrm{CO}$

$\mathrm{Val}=1.07374 \mathrm{E}-01 /$

Comp $=\mathrm{CO} 2$

$\mathrm{Val}=4.11858 \mathrm{E}-01 /$

Comp $=\mathrm{CH} 4$

Val $=5.92536 \mathrm{E}-02 /$

Comp $=\mathrm{C} 2 \mathrm{H} 2$

$\mathrm{Va}]=0.0000 \mathrm{E}-00 /$

Comp $=\mathrm{C} 2 \mathrm{H} 4$

$\mathrm{Val}=1.22623 \mathrm{E}-04 /$

Comp $=\mathrm{C} 2 \mathrm{H} 6$

$\mathrm{Va}]=8.28036 \mathrm{E}-03 /$

Comp $=$ C3H6-2

$\mathrm{Va}]=0.0000 \mathrm{E}-00 /$

Comp $=\mathrm{C} 3 \mathrm{H} 8$

$\mathrm{Va}]=0.0000 \mathrm{E}-00 /$

Comp $=$ C6H6

Val $=5.80442 \mathrm{E}-03 /$

Comp $=$ C6H6O

$\mathrm{Val}=6.59455 \mathrm{E}-03 /$

Ssid $=$ Mixed

Comp $=\mathrm{C} 10 \mathrm{H} 8$

Val $=3.87915 \mathrm{E}-04 /$

Ssid $=$ Mixed

Comp $=$ C14H10-1

VaT $=3.87915 \mathrm{E}-04 /$

Ssid $=$ Mixed

Comp $=$ C7H80-4

$V a 7=3.87915 E-04 /$

Ssid = Cisolid

Comp $=\mathrm{H} 2 \mathrm{O}$

Ssid $=$ Ncpsd

Comp $=02 S I$

$\mathrm{Val}=3.41829 \mathrm{E}-01 /$

Ssid $=$ Ncpsd

Comp $=$ Char

Comp $=$ Wood

$\mathrm{Va}]=1.53194 \mathrm{E}-02 /$

$\mathrm{Val}=2.72938 \mathrm{E}-02 /$

$\mathrm{Va}]=0.0$

;

Block Solsep Sep2

Frac Subs $=$ Ncpsd Stream $=$ Solid1 Comp $=$ Char $\quad F r a c=1.0 /$ Subs $=$ Mixed Stream=Gasl Comp $=C 14 H 10-1$ Frac $=0.9995$

Flash-specs Gas1 Pres=319.088

;

Block Solsp2 Sep2

Frac Subs $=$ Cisolid Stream $=$ Solid2 Comp $=02 S I \quad F r a c=1.0 /$ Subs $=$ Mixed Stream $=$ Gas Ib Comp $=C 14 \mathrm{H} 10-1 \quad$ Frac $=0.9995$

Flash-specs Gaslb Pres $=319.088$

;

Block Heat 1 Heatx

Param Option $=1$ Fdir $=0$ Thot $=1490$

;

Block Chcomp2 Compr

Param Type $=1$ Pres $=25 \quad E P=0.90$

;

Block Heat2 Heatx

Param Opt $=1$ Fdir $=0$ Thot $=1489.9$

;

Block Heat3 Heatx

param opt $=1$ Fdir $=0$ Thot $=1489$

;

Block CM Mixer ; 
Block Cdecomp Ryield

Param Pres $=25$ Temp $=2000$

Massyield Ssid $=$ Mixed Comp $=\mathrm{CO} 2 \quad$ Val $=0.249712543 /$

$$
\text { Ssid }=\text { Mixed Comp }=\mathrm{H} 2 \mathrm{O} \quad \mathrm{Val}=0.039009473 /
$$

Ssid $=$ Mixed Comp $=$ N2 $\quad$ Val $=0.711277984$

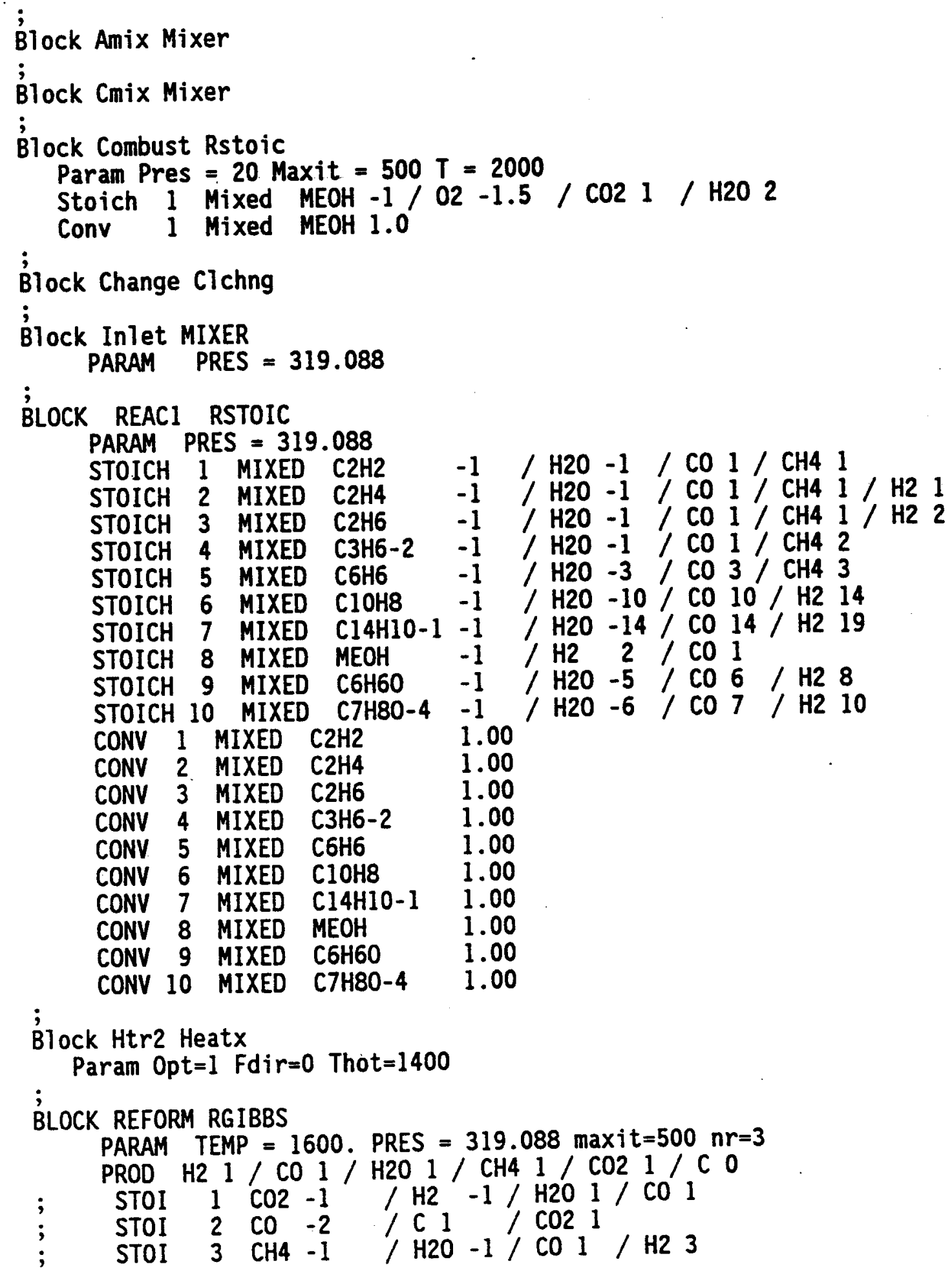




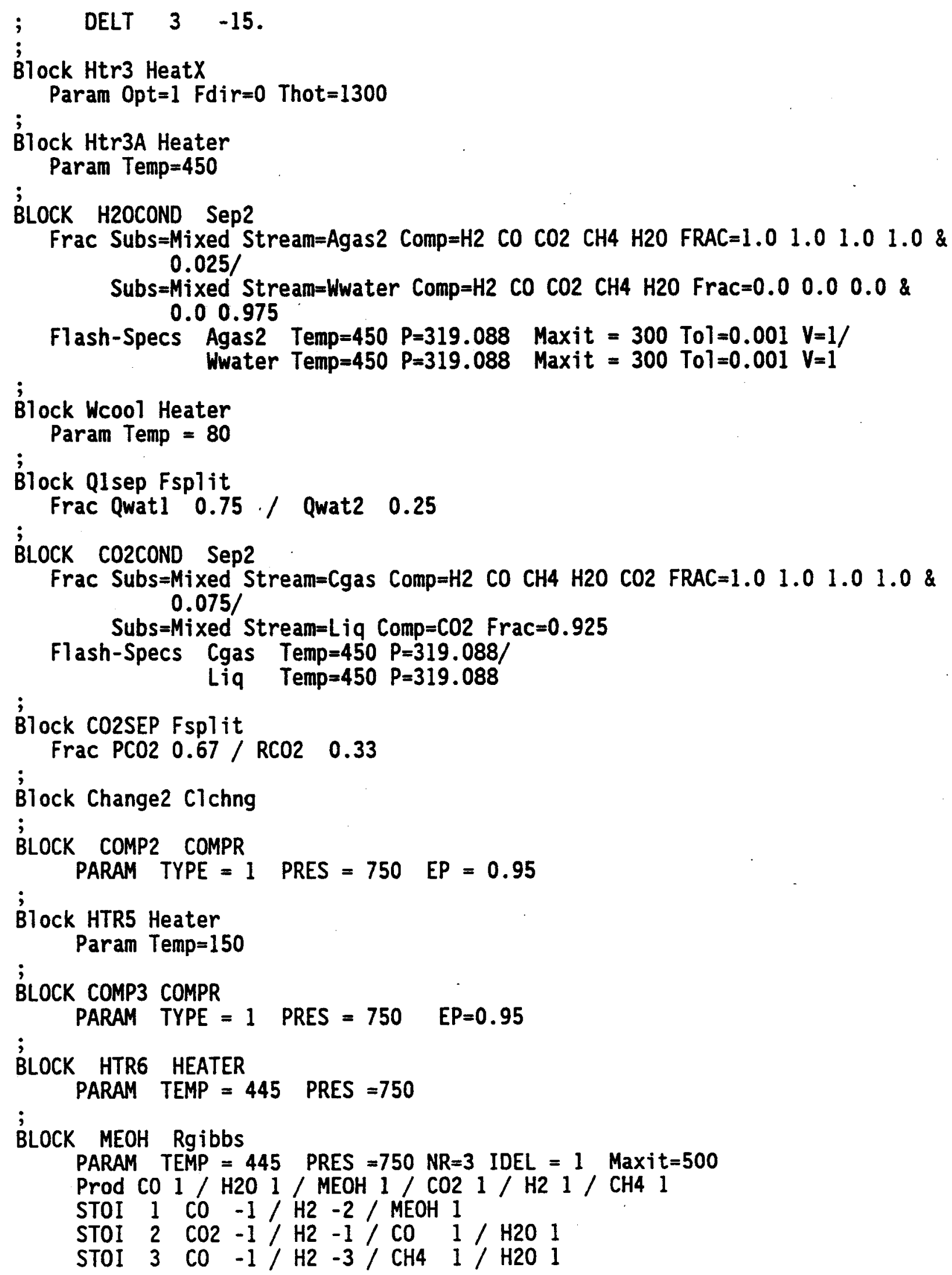




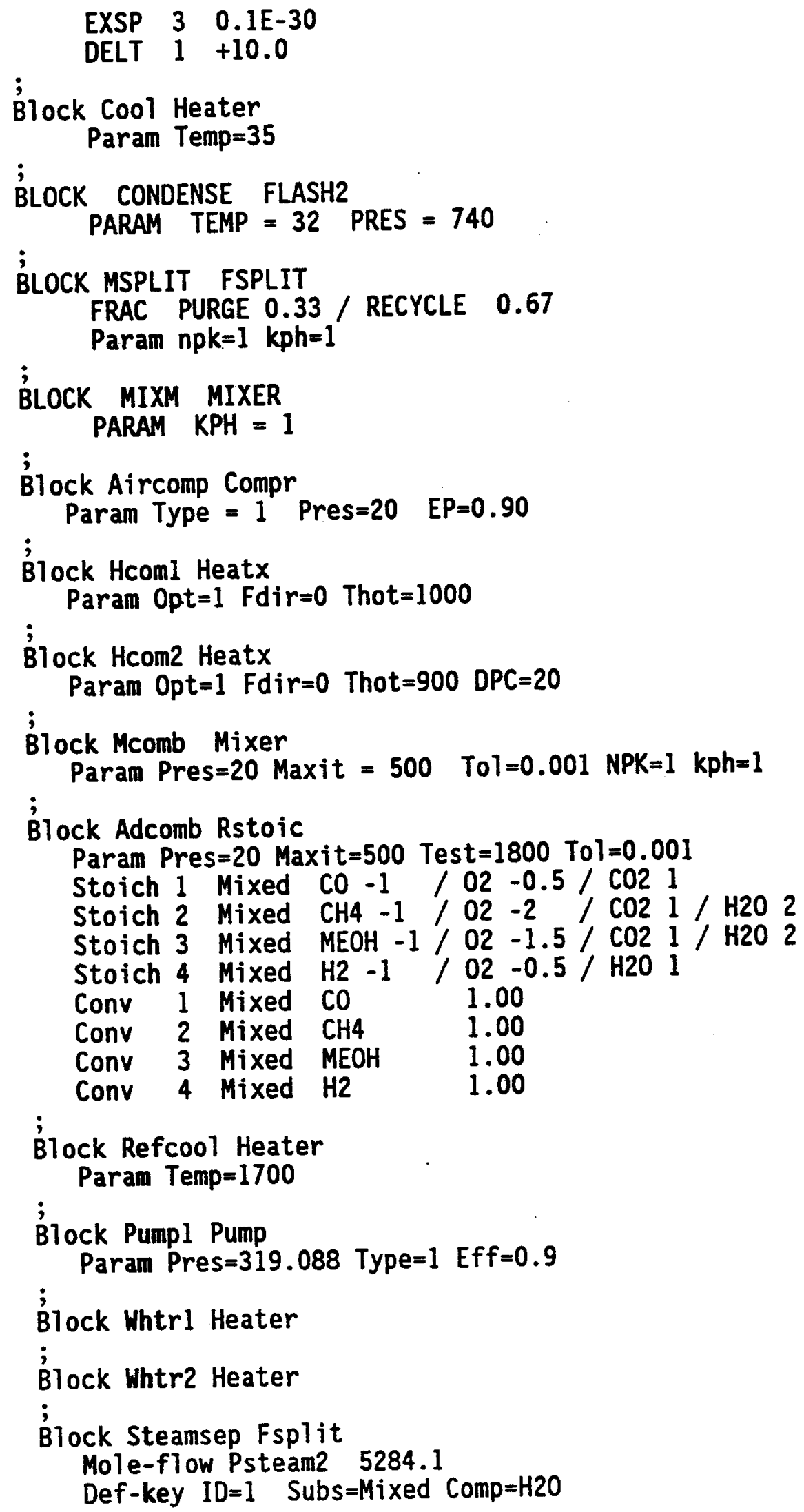




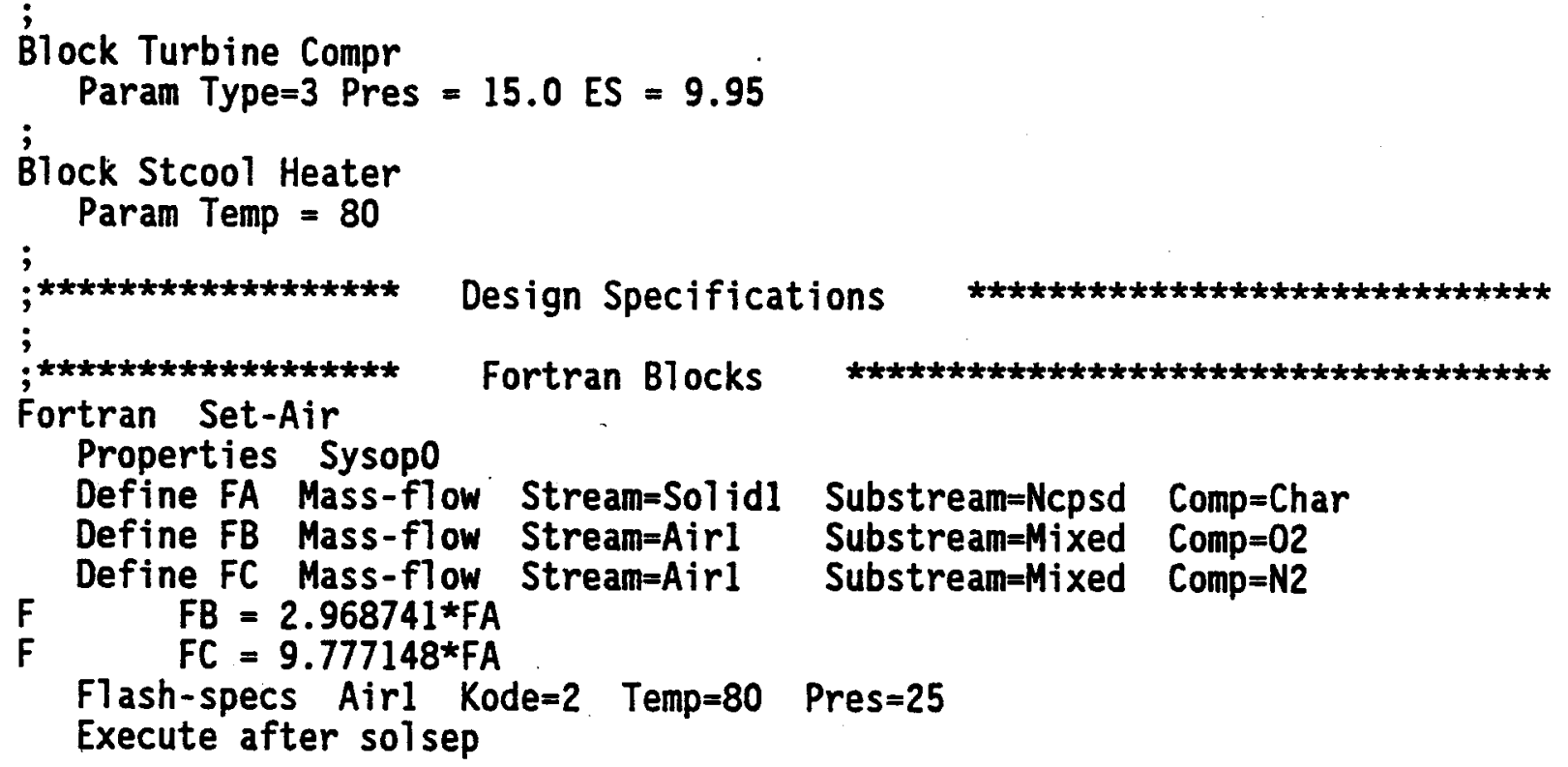


APPENDIX 6 
HAWAII MATERIAL BALANCE INFORMATION

Feed: Bagasse

$$
\begin{array}{lr}
\text { Proximate Analysis, } & \text { Dry } \\
\text { VM, } \% & 82.1 \\
\text { FC } & 14.8 \\
\text { Ash } & 3.1 \\
& -30.0
\end{array}
$$

UItimate Analysis

\begin{tabular}{lrr} 
& & \\
$C, \%$ & Dry & MAF \\
$H$ & 47.90 & 49.43 \\
$N$ & 6.20 & 6.40 \\
$S$ & 0.60 & 0.62 \\
0 & 0.01 & 0.01 \\
Ash & 42.19 & 43.54 \\
& 3.10 & \\
&..-- &.- .5 \\
\hline
\end{tabular}

\section{Heating Value}

Given

$19,100 \mathrm{~kJ} / \mathrm{kg}$

Corr. $\quad 19,021 \mathrm{~kJ} / \mathrm{kg}$

(NREL CORRELATION)

Process Information

\begin{tabular}{lrr} 
System A & System B \\
\hline Conditions & 830 & 750 \\
Temperature, C & 1526 & 1382 \\
Pressure, Bars & 22 & 10 \\
Steam/Feed, 1b/1b & 319.1 & 145.0 \\
Oxygen/Feed, Ib/1b & 0.58 & 1.2 \\
Feed Moisture, \% wet & 0.25 & 0.00 \\
& 10.60 & 5.00
\end{tabular}




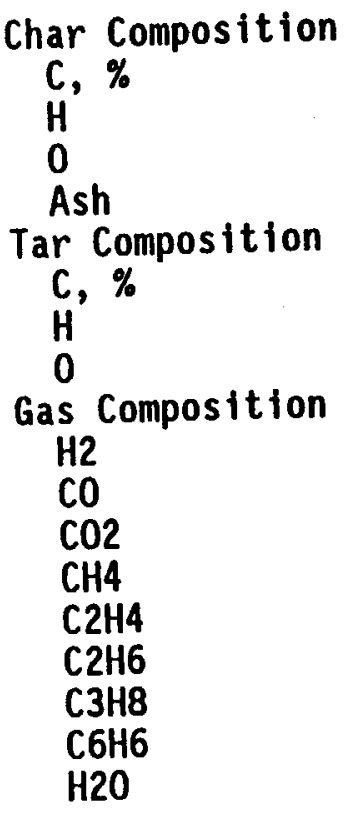

Yield Structure

Gas (m3/ $\mathrm{kg}$ bagasse) (ft3/ib bagasse)

Carbon Conversion, \% To Gas

To Char

To Liquid

* = changed from original data

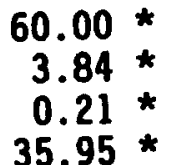

87.4

5.6

0.3

6.7

69.2

69.2

6.7

24.1

6.7

24.1

17.15

8.77

21.41

8.45

0.01

0.63

0.00

0.17

43.41 *

100.00

33.46

9.03

15.84

4.96

0.00

0.00

0.00

0.00

36.71

100.00

\subsection{5}

37.076

87.415 *

10.401 *

2.184 *

100.000

1.68

59.322

75.0

25.0

0.0 
System A Analysis

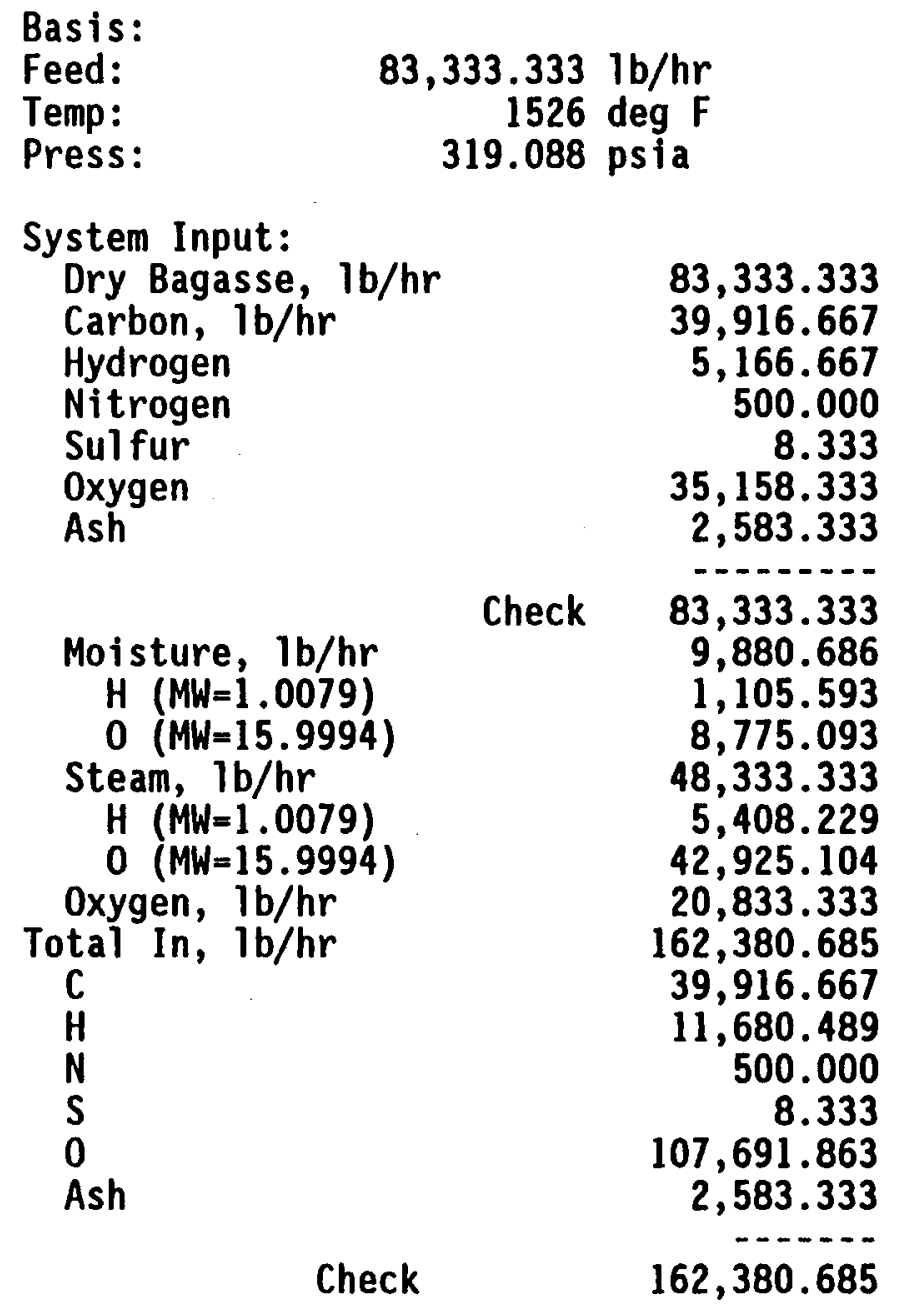




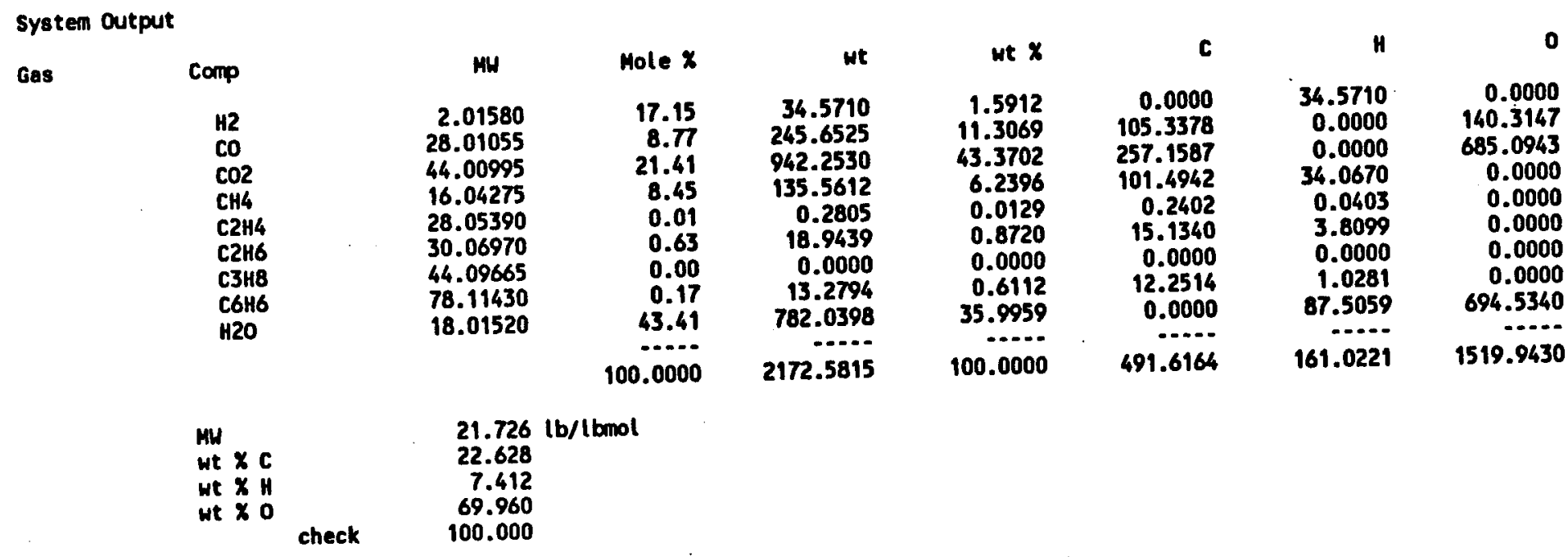


Product Yield, based on carbon conversion

$\begin{array}{lrr} & \text { Total } & \text { Carbon } \\ \text { Gas } & 154,201.985 & 34,893.154 \\ \text { Char } & 6,919.554 & 4,151.732 \\ \text { Tar } & 1,259.798 & 871.780 \\ & -1 . . . & -\ldots \\ \text { Out } & 162,381.337 & 39,916.667 \\ \text { Error, \% } & 0.000 & (0.000)\end{array}$

$\begin{array}{rr}\text { Hydrogen } & \text { Oxygen } \\ 11,428.767 & 107,880.064 \\ 265.711 & 14.531 \\ 84.406 & 303.611 \\ -2 .- & -.- \\ 11,778.885 & 108,198.206 \\ 0.842 & 0.470\end{array}$

Char Yield, based on ash and char comp 7,185.906 $\mathrm{lb} / \mathrm{hr}$

Gas Yield, based on vol and MW

$\begin{array}{lll}186,989.401 & S T= & 32 \mathrm{deg} F \\ 176,914.179 & \text { ST }= & 60 \mathrm{deg} F \\ 173,573.336 & S T= & 70 \mathrm{deg} F\end{array}$

$\begin{array}{lrr}\text { Tar Composition } & & \\ \text { Component } & \text { MW } & \text { wt \% } \\ \text { C10H8 } & 128.1747 & 5 \\ \text { C14H100 } & 178.2351 & 5 \\ \text { C7H80 } & 108.14065 & 5 \\ \text { C6H60 } & 95.0742 & 85 \\ & & 100.000 \\ & & \text { MODEL } \\ & \text { ANALYSIS } & \\ \text { WT } \% \text { C } & & 69.6 \\ \text { WT \% H } & 6.7 & 6.35 \\ \text { WT } \% \text { O } & 24.1 & 14.99\end{array}$

Reported Tar Analysis must contain water. It is much different than reported IGT analyses 
Case A

IGT input file, oxygen case

Feed
Dry feed
Moisture 1
Steam
Oxygen
Total
$83,333.333$
9,880.686 Associated water to gasifier
$48,333.333$
$20,833.333$
$162,380.685$

Dryer Moisture

\begin{tabular}{lrr}
$\begin{array}{l}\text { Product } \\
\text { Component }\end{array}$ & \multicolumn{1}{l}{ lb/hr } & $\begin{array}{c}\text { lb/lb gasifier } \\
\text { feed }\end{array}$ \\
H2 & & \\
CO & $2,453.723$ & $1.51109 \mathrm{E}-02$ \\
C02 & $17,435.529$ & $1.07374 \mathrm{E}-01$ \\
CH4 & $66,877.716$ & $4.11858 \mathrm{E}-01$ \\
C2H4 & $9,621.647$ & $5.92536 \mathrm{E}-02$ \\
C2H6 & 19.912 & $1.22623 \mathrm{E}-04$ \\
C3H8 & $1,344.570$ & $8.28036 \mathrm{E}-03$ \\
C6H6 & 0.000 & $0.00000 \mathrm{E}+00$ \\
H20 & 942.526 & $5.80442 \mathrm{E}-03$ \\
C10H8 & $55,506.362$ & $3.41829 \mathrm{E}-01$ \\
C14H100 & 62.990 & $3.87915 \mathrm{E}-04$ \\
C7H80 & 62.990 & $3.87915 \mathrm{E}-04$ \\
C6H60 & 62.990 & $3.87915 \mathrm{E}-04$ \\
Char & $1,070.828$ & $6.59455 \mathrm{E}-03$ \\
& $6,919.554$ & $4.26132 \mathrm{E}-02$ \\
Total & $-162,381.337$ & $1.00000 \mathrm{E}+00$
\end{tabular}


Case A

Air input flle

Basis: air at $80 \mathrm{deg}$ F, $14.696 \mathrm{psia,} 20 \times \mathrm{RH}$

Air Composition

$\begin{array}{lrrrr}\text { Component } & \text { MH } & \text { Mole X } & \text { Wt } & \text { Wt X } \\ \text { Oxygen } & 31.9988 & 20.66 & 661.095 & 22.94 \\ \text { Nitrogen } & 28.0134 & 77.01 & 2.157 .312 & 74.87 \\ \text { Argon } & 39.948 & 0.92 & 36.752 & 1.28 \\ \text { CO2 } & 44.00995 & 0.03 & 1.320 & 0.05 \\ \text { H2O } & 18.0152 & 1.38 & 24.861 & 0.86 \\ & & & \ldots 1 . .- & \ldots . . . \\ & & & 2,881.341 & 100.00\end{array}$

Feed

Ory feed $83,333.333$

Molsture $19,880.686$ Assoclated water to gasiffer Stean 48,333.333

Oxygen

Oxygen 20,833.333

Argonen 67,984.154

Argon $\quad 1,158.184$

$\begin{array}{rr}\text { CO2 } & 41.607 \\ H 20 & 783.453\end{array}$

Total $232,348.084$

Molsture $2 \quad 73,452.647$ Associated water removed in dryer

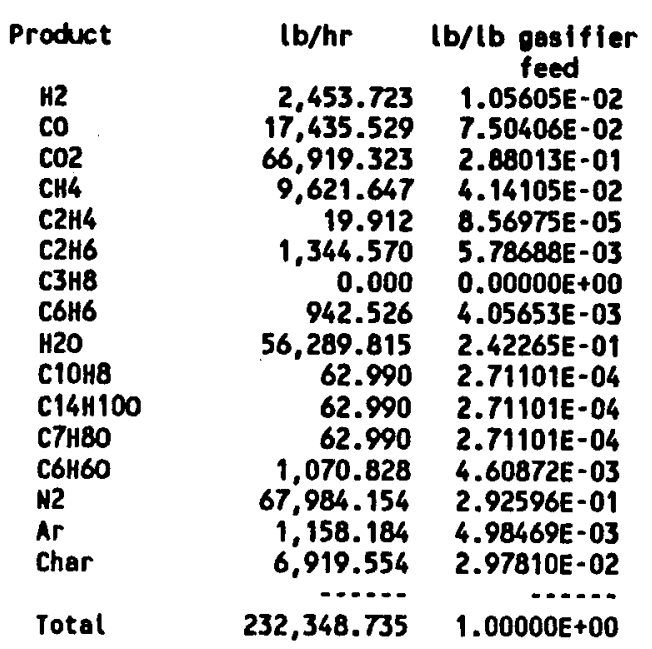


APPENDIX 7 
BCL Gasifier Data

\begin{tabular}{|c|c|c|c|c|c|c|c|c|c|c|}
\hline I tem/Test & 71 & 74 & 75 & 76 & $\pi$ & 78 & 79 & 80 & 81 & 82 \\
\hline $\begin{array}{l}\text { Gasifier Conditions } \\
\text { Temperature, deg F } \\
\text { Pressure, psig } \\
\text { Moisture, Ht } \mathrm{X} \\
\text { Wet Wood Rate, Ib/hr } \\
\text { Dry wood Rate, Ib/hr } \\
\text { Nitrogen Rate, Ib/hr } \\
\text { Steam Rate, Ib/hr } \\
\text { Steam Rate, Ib/lb BDH } \\
\text { Total H2O Rate, Ib/lb BOH } \\
\text { Total H2O, Ib/Ib MAF HOOd } \\
\text { Total H2O, SCF/lb MAF HOOd }\end{array}$ & 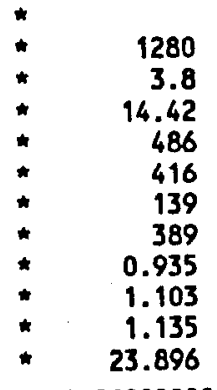 & $\begin{array}{r}1504 \\
6.2 \\
15.69 \\
588 \\
496 \\
173 \\
404 \\
0.815 \\
1.000 \\
1.012 \\
21.318\end{array}$ & $\begin{array}{r}1562 \\
8.2 \\
6.88 \\
700 \\
652 \\
315 \\
401 \\
0.615 \\
0.689 \\
0.693 \\
14.595\end{array}$ & $\begin{array}{r}1359 \\
8.4 \\
9.00 \\
916 \\
834 \\
169 \\
461 \\
0.553 \\
0.651 \\
0.659 \\
13.870\end{array}$ & $\begin{array}{r}1466 \\
7.8 \\
9.00 \\
777 \\
707 \\
204 \\
415 \\
0.587 \\
0.686 \\
0.694 \\
14.614\end{array}$ & $\begin{array}{r}1382 \\
7.0 \\
10.79 \\
1046 \\
933 \\
218 \\
371 \\
0.398 \\
0.519 \\
0.530 \\
11.151\end{array}$ & $\begin{array}{r}1445 \\
5.8 \\
10.00 \\
786 \\
707 \\
160 \\
406 \\
0.574 \\
0.686 \\
0.688 \\
14.499\end{array}$ & $\begin{array}{r}1470 \\
5.2 \\
10.00 \\
580 \\
522 \\
160 \\
412 \\
0.789 \\
0.900 \\
0.908 \\
19.128\end{array}$ & $\begin{array}{r}1548 \\
5.0 \\
8.12 \\
408 \\
375 \\
160 \\
413 \\
1.101 \\
1.189 \\
1.195 \\
25.158\end{array}$ & $\begin{array}{r}1429 \\
6.8 \\
8.12 \\
782 \\
719 \\
152 \\
437 \\
0.608 \\
0.695 \\
0.698 \\
14.710\end{array}$ \\
\hline $\begin{array}{l}\text { Feed } \\
\text { Type } \\
\text { Size }\end{array}$ & $\begin{array}{r}* \quad \text { Red Oak } \\
\text { * chips }\end{array}$ & $\begin{array}{r}\text { Red Oak } \\
\text { chips }\end{array}$ & $\begin{array}{r}\text { Red Oak } \\
\text { chips }\end{array}$ & $\begin{array}{r}\text { Red Oak } \\
\text { chips }\end{array}$ & $\begin{array}{l}\text { Red Oak } \\
\text { chips }\end{array}$ & $\begin{array}{c}\text { Red Oak } \\
\text { chips }\end{array}$ & $\begin{array}{c}\text { Bir+Map } \\
\text { chips }\end{array}$ & $\begin{array}{r}\text { BIr+Map } \\
\text { chips }\end{array}$ & $\begin{array}{c}\text { BirtMap } \\
\text { chips }\end{array}$ & $\begin{array}{r}\text { BirtHap } \\
\text { chips }\end{array}$ \\
\hline $\begin{array}{l}\text { Size } \\
\text { Proximate Analysis } \\
\text { Volatile Matter, wt } x \text { dry } \\
\text { Ash } \\
\text { Fixed Carbon }\end{array}$ & $\begin{array}{rr}* & 81.07 \\
* & 2.76 \\
* & 16.17\end{array}$ & $\begin{array}{r}70.42 \\
1.21 \\
28.37\end{array}$ & $\begin{array}{r}82.82 \\
0.63 \\
16.55\end{array}$ & $\begin{array}{r}83.38 \\
1.14 \\
15.48\end{array}$ & $\begin{array}{r}83.38 \\
1.14 \\
15.48\end{array}$ & $\begin{array}{r}80.47 \\
2.03 \\
17.50\end{array}$ & $\begin{array}{r}83.99 \\
0.36 \\
15.65\end{array}$ & $\begin{array}{r}84.48 \\
0.87 \\
14.65\end{array}$ & $\begin{array}{r}83.63 \\
0.44 \\
15.93\end{array}$ & $\begin{array}{r}83.30 \\
0.44 \\
15.93\end{array}$ \\
\hline $\begin{array}{l}\text { Proximate Analysis } \\
\text { Molsture, Ht } x \\
\text { Volatlle Matter } \\
\text { Ash } \\
\text { Fixed Carbon }\end{array}$ & $\begin{array}{r}* \quad 14.42 \\
* \quad 69.38 \\
* \quad 2.36 \\
* \quad 13.84\end{array}$ & $\begin{array}{r}15.69 \\
59.37 \\
1.02 \\
23.92\end{array}$ & $\begin{array}{r}6.88 \\
77.12 \\
0.59 \\
15.41\end{array}$ & $\begin{array}{r}9.00 \\
75.88 \\
1.04 \\
14.09\end{array}$ & $\begin{array}{r}9.00 \\
75.88 \\
1.04 \\
14.09\end{array}$ & $\begin{array}{r}10.79 \\
71.79 \\
1.81 \\
15.61\end{array}$ & $\begin{array}{r}10.00 \\
75.59 \\
0.32 \\
14.09\end{array}$ & $\begin{array}{r}10.00 \\
76.03 \\
0.78 \\
13.19\end{array}$ & $\begin{array}{r}8.12 \\
76.84 \\
0.40 \\
14.64\end{array}$ & $\begin{array}{r}8.12 \\
76.54 \\
0.40 \\
14.64\end{array}$ \\
\hline $\begin{array}{l}\text { Ultimate Analysis } \\
\text { Ash, wt } x \text { dry } \\
\text { Carbon } \\
\text { Hydrogen } \\
\text { Nitrogen } \\
\text { Chlorine } \\
\text { Sulfur } \\
\text { Oxygen (diff) }\end{array}$ & $\begin{array}{r}\text { * } \quad 2.76 \\
* \quad 49.34 \\
\star \quad 5.93 \\
* \quad 0.07 \\
* \quad 0.03 \\
* \quad 0.13 \\
* \quad 41.74\end{array}$ & $\begin{array}{r}1.21 \\
57.75 \\
5.58 \\
0.44 \\
0.03 \\
0.87 \\
34.12\end{array}$ & $\begin{array}{r}0.63 \\
50.08 \\
6.05 \\
0.21 \\
0.08 \\
0.00 \\
42.95\end{array}$ & $\begin{array}{r}1.14 \\
49.77 \\
5.99 \\
0.20 \\
0.02 \\
0.00 \\
42.88\end{array}$ & $\begin{array}{r}1.14 \\
49.77 \\
5.99 \\
0.20 \\
0.02 \\
0.00 \\
42.88\end{array}$ & $\begin{array}{r}2.03 \\
49.50 \\
5.86 \\
0.25 \\
0.03 \\
0.01 \\
42.32\end{array}$ & $\begin{array}{r}0.36 \\
49.85 \\
6.12 \\
0.10 \\
0.03 \\
0.08 \\
43.46\end{array}$ & $\begin{array}{r}0.87 \\
50.14 \\
6.10 \\
0.03 \\
0.03 \\
0.03 \\
42.80\end{array}$ & $\begin{array}{r}0.44 \\
51.13 \\
5.73 \\
0.14 \\
0.02 \\
0.03 \\
42.51\end{array}$ & $\begin{array}{r}0.44 \\
51.13 \\
5.73 \\
0.14 \\
0.02 \\
0.03 \\
42.51\end{array}$ \\
\hline
\end{tabular}




\begin{tabular}{|c|c|c|c|c|c|c|c|c|c|c|}
\hline 1 tem/Test & 71 & $\begin{array}{c}74 \\
7 .\end{array}$ & 75 & 76 & 77 & 78 & 79 & 80 & 81 & 82 \\
\hline $\begin{array}{l}\text { Product Gas } \\
\text { Rate, SCF/lb MAF } \\
\text { Rate, SCF/ Ib BDW }\end{array}$ & $\begin{array}{l}7.66 \\
7.45\end{array}$ & $\begin{array}{l}10.74 \\
10.61\end{array}$ & $\begin{array}{l}13.94 \\
13.85\end{array}$ & $\begin{array}{l}8.50 \\
8.40\end{array}$ & $\begin{array}{l}10.84 \\
10.72\end{array}$ & $\begin{array}{l}9.94 \\
9.74\end{array}$ & $\begin{array}{l}10.25 \\
10.21\end{array}$ & $\begin{array}{l}10.59 \\
10.50\end{array}$ & $\begin{array}{l}11.08 \\
11.03\end{array}$ & $\begin{array}{l}9.82 \\
9.78\end{array}$ \\
\hline $\begin{array}{l}\text { Dry H2 free Comp } \\
\text { H2, mole } x \\
\text { CO } \\
\text { CO2 } \\
\mathrm{CH}_{4} \\
\mathrm{C} 2 \mathrm{H} 2 \\
\mathrm{C} 2 \mathrm{H4} \\
\mathrm{C} 2 \mathrm{H} 6\end{array}$ & $\begin{array}{r}13.25 \\
49.52 \\
16.32 \\
16.10 \\
0.00 \\
3.81 \\
1.00 \\
\ldots \ldots\end{array}$ & $\begin{array}{r}20.59 \\
43.98 \\
14.24 \\
15.90 \\
0.00 \\
4.80 \\
0.49 \\
\cdots\end{array}$ & $\begin{array}{r}20.51 \\
43.80 \\
17.08 \\
14.41 \\
0.00 \\
3.94 \\
0.26 \\
\ldots \ldots . .\end{array}$ & $\begin{array}{r}14.66 \\
49.18 \\
14.39 \\
16.59 \\
0.00 \\
4.18 \\
1.00 \\
\ldots \ldots\end{array}$ & $\begin{array}{r}18.15 \\
46.27 \\
13.93 \\
16.30 \\
0.00 \\
4.63 \\
0.72 \\
\cdots \ldots . .\end{array}$ & $\begin{array}{r}14.94 \\
49.13 \\
14.21 \\
16.47 \\
0.00 \\
4.30 \\
0.95 \\
\ldots \ldots . .\end{array}$ & $\begin{array}{l}17.20 \\
48.15 \\
12.75 \\
16.38 \\
0.00 \\
4.78 \\
0.74 \\
\cdots \ldots\end{array}$ & $\begin{array}{l}20.81 \\
44.17 \\
14.78 \\
15.36 \\
0.00 \\
4.28 \\
0.60 \\
\ldots \ldots\end{array}$ & $\begin{array}{r}23.79 \\
41.91 \\
15.42 \\
14.42 \\
0.00 \\
4.15 \\
0.31 \\
\ldots . . .\end{array}$ & $\begin{array}{l}19.36 \\
45.23 \\
14.57 \\
15.83 \\
0.00 \\
4.31 \\
0.70 \\
\ldots \ldots\end{array}$ \\
\hline & $\star \quad 100.00$ & 100.00 & 100.00 & 100.00 & 100.00 & 100.00 & 100.00 & 100.00 & 100.00 & 100.00 \\
\hline $\begin{array}{l}\text { MH, Ib/lbmole } \\
H H V, \text { Btu/lb mole } \\
H H V, \text { Btu/lb } \\
\text { HHV, Btu/SCF a } 60 \mathrm{deg} F\end{array}$ & $\begin{array}{r}25.273 \\
* \quad 167,980.25 \\
* \quad 6,646.70 \\
* \quad 442.75\end{array}$ & $\begin{array}{r}23.046 \\
172,079.84 \\
7,466.86 \\
453.56\end{array}$ & $\begin{array}{r}23.694 \\
159,299.31 \\
6,723.13 \\
419.87\end{array}$ & $\begin{array}{r}24.539 \\
173.419 .53 \\
7.067 .12 \\
457.09\end{array}$ & $\begin{array}{r}23.587 \\
173,909.87 \\
7,373.04 \\
458.38\end{array}$ & $\begin{array}{r}24.451 \\
173,636.13 \\
7,101.47 \\
457.66\end{array}$ & $\begin{array}{r}23.636 \\
176,380.20 \\
7,462.25 \\
464.89\end{array}$ & $\begin{array}{r}23.142 \\
168,097.18 \\
7,263.83 \\
443.06\end{array}$ & $\begin{array}{r}22.576 \\
162,677.77 \\
7,205.81 \\
428.78\end{array}$ & $\begin{array}{r}23.431 \\
170,256.79 \\
7,266.36 \\
448.75\end{array}$ \\
\hline $\begin{array}{l}\text { c, lb/lbmole } \\
c, \text { lb/lb gas } \\
\text { c, lb/scF } \\
\text { c, lb/lb maf nood } \\
\text { c Conv to Gas, } x\end{array}$ & $\begin{array}{r}10.9974 \\
\star \quad 0.4351 \\
\star \quad 0.0290 \\
\star \quad 0.2220 \\
\star \quad 43.7590\end{array}$ & $\begin{array}{r}10.1734 \\
0.4414 \\
0.0268 \\
0.2880 \\
49.2647\end{array}$ & $\begin{array}{r}10.0521 \\
0.4242 \\
0.0265 \\
0.3693 \\
73.2849\end{array}$ & $\begin{array}{r}10.8725 \\
0.4431 \\
0.0287 \\
0.2436 \\
48.3842\end{array}$ & $\begin{array}{r}10.4737 \\
0.4440 \\
0.0276 \\
0.2992 \\
59.4410\end{array}$ & $\begin{array}{r}10.8473 \\
0.4436 \\
0.0286 \\
0.2842 \\
56.2467\end{array}$ & $\begin{array}{r}10.6082 \\
0.4488 \\
0.0280 \\
0.2866 \\
57.2847\end{array}$ & $\begin{array}{r}10.0978 \\
0.4363 \\
0.0266 \\
0.2819 \\
55.7244\end{array}$ & $\begin{array}{r}9.6894 \\
0.4292 \\
0.0255 \\
0.2830 \\
55.0996\end{array}$ & $\begin{array}{r}10.2875 \\
0.4391 \\
0.0271 \\
0.2663 \\
51.8484\end{array}$ \\
\hline $\begin{array}{l}\text { H, Ib/lb mole } \\
H, \text { Ib/lb gas } \\
H, \text { Ib/scF } \\
\text { H, Ib/lb maF wood } \\
\text { H Conv to Gas, wt } x\end{array}$ & $\begin{array}{r}* \quad 1.1303 \\
* \quad 0.0447 \\
* \quad 0.0030 \\
* \quad 0.0228 \\
* \quad 37.4196\end{array}$ & $\begin{array}{r}1.2792 \\
0.0555 \\
0.0034 \\
0.0362 \\
64.1111\end{array}$ & $\begin{array}{r}1.1690 \\
0.0493 \\
0.0031 \\
0.0430 \\
70.5449\end{array}$ & $\begin{array}{r}1.1934 \\
0.0486 \\
0.0031 \\
0.0267 \\
44.1250\end{array}$ & $\begin{array}{r}1.2532 \\
0.0531 \\
0.0033 \\
0.0358 \\
59.0955\end{array}$ & $\begin{array}{r}1.1960 \\
0.0489 \\
0.0032 \\
0.0313 \\
52.3849\end{array}$ & $\begin{array}{r}1.2446 \\
0.0527 \\
0.0033 \\
0.0336 \\
54.7423\end{array}$ & $\begin{array}{r}1.2476 \\
0.0539 \\
0.0033 \\
0.0348 \\
56.5903\end{array}$ & $\begin{array}{r}1.2470 \\
0.0552 \\
0.0033 \\
0.0364 \\
63.2747\end{array}$ & $\begin{array}{r}1.2446 \\
0.0531 \\
0.0033 \\
0.0322 \\
55.9704\end{array}$ \\
\hline $\begin{array}{l}\text { Tar Estimate } \\
\text { Yield, Ib/lb maf feed } \\
\text { Eq Tar }=0.046-.00002^{\star} \text { Tenp }\end{array}$ & $\stackrel{\star}{\star}$ & 0.0159 & 0.0148 & 0.0188 & 0.0167 & 0.0184 & 0.0171 & 0.0166 & 0.0150 & 0.0174 \\
\hline $\begin{array}{l}\text { Comp } \\
\text { C3H6, LB/Ib maf wood } \\
\text { C6H6 } \\
\text { C1OH8 } \\
\text { C14H1O }\end{array}$ & $\begin{array}{ll}* & 0.0051 \\
* & 0.0051 \\
* \quad 0.0051 \\
* \quad 0.0051\end{array}$ & $\begin{array}{l}0.00398 \\
0.00398 \\
0.00398 \\
0.00398\end{array}$ & $\begin{array}{l}0.00369 \\
0.00369 \\
0.00369 \\
0.00369\end{array}$ & $\begin{array}{l}0.004705 \\
0.004705 \\
0.004705 \\
0.004705\end{array}$ & $\begin{array}{l}0.00417 \\
0.00417 \\
0.00417 \\
0.00417\end{array}$ & $\begin{array}{l}0.00459 \\
0.00459 \\
0.00459 \\
0.00459\end{array}$ & $\begin{array}{l}0.004275 \\
0.004275 \\
0.004275 \\
0.004275\end{array}$ & $\begin{array}{l}0.00415 \\
0.00415 \\
0.00415 \\
0.00415\end{array}$ & $\begin{array}{l}0.00376 \\
0.00376 \\
0.00376 \\
0.00376\end{array}$ & $\begin{array}{l}0.004355 \\
0.004355 \\
0.004355 \\
0.004355\end{array}$ \\
\hline $\begin{array}{l}\text { Ib } c / \text { lb maf wood } \\
\text { lb } H / \text { lb maf wood } \\
\text { lb } c / l b \text { dry wood } \\
\text { lb } H / l b \text { dry wood }\end{array}$ & $\begin{array}{l}0.0147 \\
\star \quad 0.0057 \\
\quad 0.0143 \\
\star \quad 0.0055\end{array}$ & $\begin{array}{l}0.0115 \\
0.0044 \\
0.0114 \\
0.0044\end{array}$ & $\begin{array}{l}0.0107 \\
0.0041 \\
0.0106 \\
0.0041\end{array}$ & $\begin{array}{l}0.0136 \\
0.0052 \\
0.0134 \\
0.0052\end{array}$ & $\begin{array}{l}0.0120 \\
0.0046 \\
0.0119 \\
0.0046\end{array}$ & $\begin{array}{l}0.0133 \\
0.0051 \\
0.0130 \\
0.0050\end{array}$ & $\begin{array}{l}0.0123 \\
0.0048 \\
0.0123 \\
0.0047\end{array}$ & $\begin{array}{l}0.0120 \\
0.0046 \\
0.0119 \\
0.0046\end{array}$ & $\begin{array}{l}0.0109 \\
0.0042 \\
0.0108 \\
0.0042\end{array}$ & $\begin{array}{l}0.0126 \\
0.0048 \\
0.0125 \\
0.0048\end{array}$ \\
\hline
\end{tabular}




\begin{tabular}{|c|c|c|c|c|c|c|c|c|c|c|}
\hline I tem/Test & 71 & 74 & 75 & 76 & $\pi$ & 78 & 79 & 80 & 81 & 82 \\
\hline $\begin{array}{l}\text { Basis: } 1 \text { lb dry wood } \\
\text { Char estimate, Let } C=86, H=4 \text {, } \\
\text { Ib Ash }\end{array}$ & $\begin{array}{c}0=10 \text { wt } \% \text { HAI } \\
0.0276\end{array}$ & 0.0121 & 0.0063 & 0.0114 & 0.0114 & 0.0203 & 0.0036 & 0.0087 & 0.0044 & 0.0044 \\
\hline $\begin{array}{l}\text { In } \\
\text { Out in Gas } \\
\text { Out in Tar } \\
\text { Out in Char }\end{array}$ & $\begin{array}{ll}\star \quad 0.4934 \\
\star \quad 0.2159 \\
\star \quad 0.0143 \\
\star \quad 0.2632\end{array}$ & $\begin{array}{l}0.5775 \\
0.2845 \\
0.0114 \\
0.2816\end{array}$ & $\begin{array}{l}0.5008 \\
0.3670 \\
0.0106 \\
0.1232\end{array}$ & $\begin{array}{l}0.4977 \\
0.2408 \\
0.0134 \\
0.2435\end{array}$ & $\begin{array}{l}0.4977 \\
0.2958 \\
0.0119 \\
0.1900\end{array}$ & $\begin{array}{l}0.4950 \\
0.2784 \\
0.0130 \\
0.2036\end{array}$ & $\begin{array}{l}0.4985 \\
0.2856 \\
0.0123 \\
0.2006\end{array}$ & $\begin{array}{l}0.5014 \\
0.2794 \\
0.0119 \\
0.2101\end{array}$ & $\begin{array}{l}0.5113 \\
0.2817 \\
0.0108 \\
0.2188\end{array}$ & $\begin{array}{l}0.5113 \\
0.2651 \\
0.0125 \\
0.2337\end{array}$ \\
\hline $\begin{array}{l}H \text { in char } \\
H \text { in gas } \\
H \text { in tar } \\
H \text { in } \\
H \text { in steam } \\
\text { Ib }{ }^{2} 20\end{array}$ & $\begin{array}{ll}\star & 0.0188 \\
\star \quad 0.0222 \\
\star \quad 0.0055 \\
\star \quad 0.1828 \\
* \quad 0.1363 \\
\star \quad 1.2177\end{array}$ & $\begin{array}{l}0.0201 \\
0.0358 \\
0.0044 \\
0.1677 \\
0.1074 \\
0.9601\end{array}$ & $\begin{array}{l}0.0088 \\
0.0427 \\
0.0041 \\
0.1376 \\
0.0820 \\
0.7328\end{array}$ & $\begin{array}{l}0.0174 \\
0.0264 \\
0.0052 \\
0.1328 \\
0.0838 \\
0.7485\end{array}$ & $\begin{array}{l}0.0136 \\
0.0354 \\
0.0046 \\
0.1367 \\
0.0831 \\
0.7427\end{array}$ & $\begin{array}{l}0.0145 \\
0.0307 \\
0.0050 \\
0.1166 \\
0.0664 \\
0.5935\end{array}$ & $\begin{array}{l}0.0143 \\
0.0335 \\
0.0047 \\
0.1380 \\
0.0854 \\
0.7631\end{array}$ & $\begin{array}{l}0.0150 \\
0.0345 \\
0.0046 \\
0.1617 \\
0.1076 \\
0.9620\end{array}$ & $\begin{array}{l}0.0156 \\
0.0363 \\
0.0042 \\
0.1904 \\
0.1343 \\
1.2005\end{array}$ & $\begin{array}{l}0.0167 \\
0.0321 \\
0.0048 \\
0.1351 \\
0.0815 \\
0.7286\end{array}$ \\
\hline $\begin{array}{l}\text { Lb in } \\
\text { Dry Hood } \\
\text { Hater }\end{array}$ & $\begin{array}{r}1.0000 \\
1.1034\end{array}$ & $\begin{array}{l}1.0000 \\
1.0000\end{array}$ & $\begin{array}{l}1.0000 \\
0.6887\end{array}$ & $\begin{array}{l}1.0000 \\
0.6511\end{array}$ & $\begin{array}{l}1.0000 \\
0.6860\end{array}$ & $\begin{array}{l}1.0000 \\
0.5188\end{array}$ & $\begin{array}{l}1.0000 \\
0.6860\end{array}$ & $\begin{array}{l}1.0000 \\
0.9004\end{array}$ & $\begin{array}{l}1.0000 \\
1.1893\end{array}$ & $\begin{array}{l}1.0000 \\
0.6954\end{array}$ \\
\hline $\begin{array}{l}\text { tb out } \\
\text { Gas } \\
\text { Tar } \\
\text { Char } \\
\text { H2O } \\
\text { Closure }\end{array}$ & $\begin{array}{l} \\
* \quad 0.4962 \\
* \quad 0.0198 \\
* \quad 0.3372 \\
* \quad 1.2177 \\
\quad 98.46\end{array}$ & $\begin{array}{l}0.6445 \\
0.0157 \\
0.3434 \\
0.9601 \\
98.19\end{array}$ & $\begin{array}{l}0.8651 \\
0.0147 \\
0.1512 \\
0.7328 \\
104.45\end{array}$ & $\begin{array}{l}0.5435 \\
0.0186 \\
0.2978 \\
0.7485 \\
97.42\end{array}$ & $\begin{array}{r}0.6662 \\
0.0165 \\
0.2349 \\
0.7427 \\
98.48\end{array}$ & $\begin{array}{l}0.6276 \\
0.0180 \\
0.2598 \\
0.5935 \\
98.69\end{array}$ & $\begin{array}{r}0.6363 \\
0.0170 \\
0.2396 \\
0.7631 \\
98.22\end{array}$ & $\begin{array}{l}0.6403 \\
0.0165 \\
0.2559 \\
0.9620 \\
98.65\end{array}$ & $\begin{array}{r}0.6564 \\
0.0150 \\
0.2618 \\
1.2005 \\
97.46\end{array}$ & $\begin{array}{l}0.6038 \\
0.0173 \\
0.2793 \\
0.7286 \\
96.09\end{array}$ \\
\hline $\begin{array}{l}\text { Water Conversion } x \\
\text { Nater Conver., } 1 \mathrm{~b} / \mathrm{lb} \text { dry nood }\end{array}$ & $\begin{array}{r}-10.37 \\
-0.1144\end{array}$ & $\begin{array}{r}3.99 \\
0.0399\end{array}$ & $\begin{array}{r}-6.41 \\
-0.0442\end{array}$ & $\begin{array}{r}-14.97 \\
-0.0975\end{array}$ & $\begin{array}{r}-8.27 \\
-0.0567\end{array}$ & $\begin{array}{r}-14.40 \\
-0.0747\end{array}$ & $\begin{array}{r}-11.24 \\
-0.0771\end{array}$ & $\begin{array}{r}-6.84 \\
-0.0616\end{array}$ & $\begin{array}{r}-0.94 \\
-0.0112\end{array}$ & $\begin{array}{r}-4.77 \\
-0.0332\end{array}$ \\
\hline
\end{tabular}




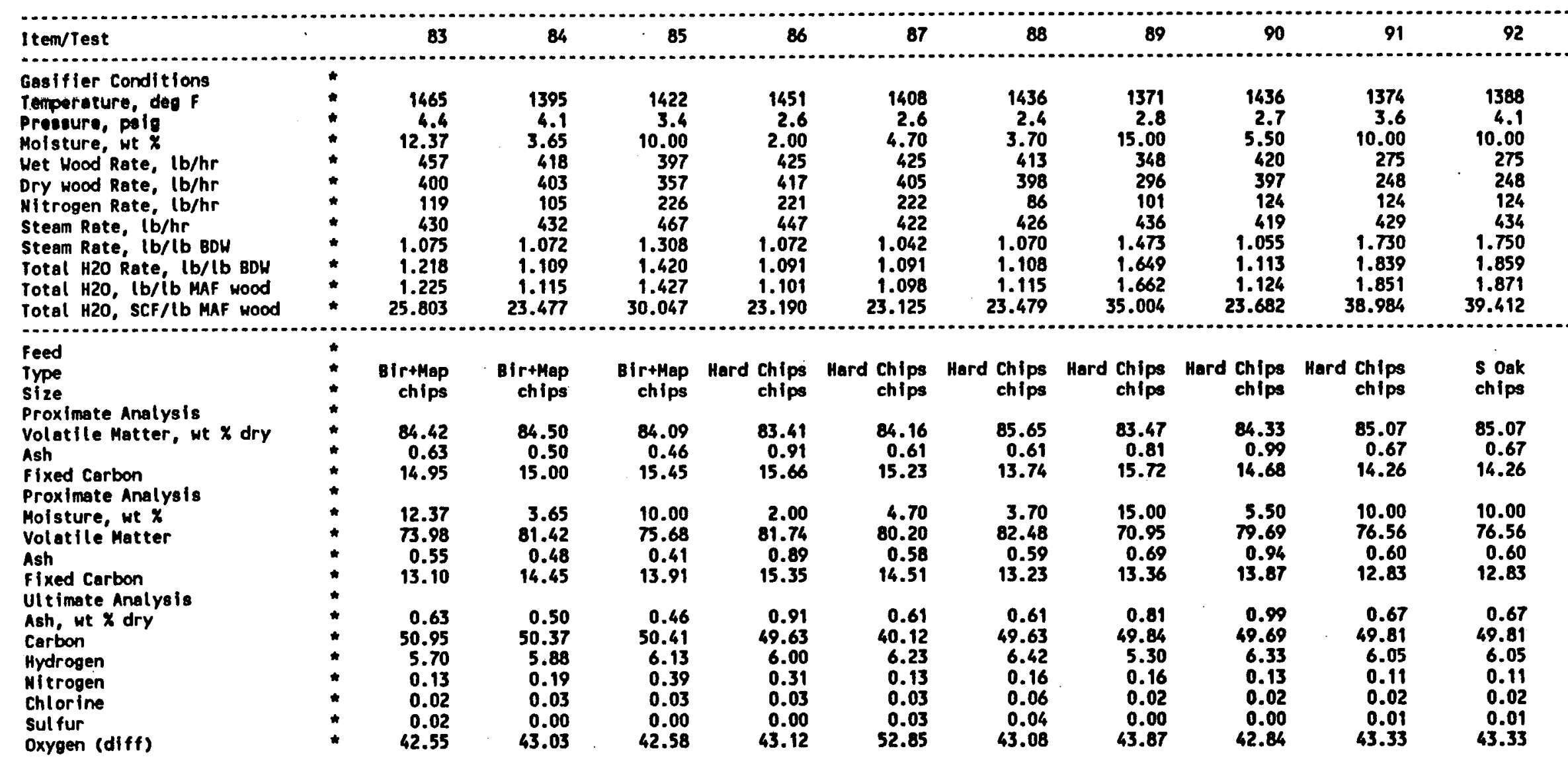




\begin{tabular}{|c|c|c|c|c|c|c|c|c|c|c|}
\hline Iten/Test & $\begin{array}{c}83 \\
-\cdots .\end{array}$ & 84 & 85 & 86 & 87 & 88 & 89 & 90 & 91 & 92 \\
\hline $\begin{array}{l}\text { Product Gas } \\
\text { Rate, SCF/Ib MAF } \\
\text { Rate, SCF/ Ib BDH }\end{array}$ & $\begin{array}{l}8.97 \\
8.91\end{array}$ & $\begin{array}{l}10.96 \\
10.91\end{array}$ & $\begin{array}{l}8.24 \\
8.20\end{array}$ & $\begin{array}{l}10.72 \\
10.62\end{array}$ & $\begin{array}{l}10.30 \\
10.24\end{array}$ & $\begin{array}{l}10.28 \\
10.22\end{array}$ & $\begin{array}{l}10.21 \\
10.13\end{array}$ & $\begin{array}{r}10.04 \\
9.94\end{array}$ & $\begin{array}{l}9.98 \\
9.91\end{array}$ & $\begin{array}{l}9.75 \\
9.68\end{array}$ \\
\hline $\begin{array}{l}\text { Dry H2 Free Comp } \\
\text { H2, mole } x \\
\text { CO } \\
\mathrm{CO} 2 \\
\mathrm{CH} 4 \\
\mathrm{C} 2 \mathrm{H} 2 \\
\mathrm{C} 2 \mathrm{H} 4 \\
\mathrm{C} 2 \mathrm{H} 6\end{array}$ & $\begin{array}{r}* \\
* \quad 20.92 \\
* \quad 42.57 \\
\star \quad 15.30 \\
\star \quad 15.91 \\
* \quad 0.00 \\
* \quad 4.88 \\
* \quad 0.42\end{array}$ & $\begin{array}{r}19.81 \\
42.23 \\
16.11 \\
15.75 \\
0.00 \\
4.83 \\
1.27 \\
\ldots \ldots\end{array}$ & $\begin{array}{r}19.19 \\
43.61 \\
14.82 \\
17.13 \\
0.00 \\
4.48 \\
0.77 \\
\ldots\end{array}$ & $\begin{array}{r}18.41 \\
46.46 \\
12.65 \\
17.06 \\
0.00 \\
4.79 \\
0.63 \\
\ldots\end{array}$ & $\begin{array}{r}16.65 \\
47.38 \\
13.26 \\
17.01 \\
0.00 \\
4.89 \\
0.81 \\
\ldots \ldots\end{array}$ & $\begin{array}{r}18.54 \\
45.36 \\
13.62 \\
16.65 \\
0.28 \\
4.83 \\
0.72 \\
\ldots \ldots\end{array}$ & $\begin{array}{r}18.21 \\
44.51 \\
15.04 \\
16.61 \\
0.23 \\
4.53 \\
0.87 \\
\ldots \ldots\end{array}$ & $\begin{array}{r}20.47 \\
43.51 \\
14.27 \\
16.12 \\
0.27 \\
4.65 \\
0.71 \\
\ldots \ldots . .\end{array}$ & $\begin{array}{r}19.38 \\
43.80 \\
14.95 \\
16.42 \\
0.24 \\
4.41 \\
0.80 \\
\end{array}$ & $\begin{array}{r}20.04 \\
43.01 \\
14.92 \\
16.58 \\
0.24 \\
4.51 \\
0.70 \\
\end{array}$ \\
\hline & * $\quad 100.00$ & 100.00 & 900.00 & 100.00 & 100.00 & 100.00 & 100.00 & 100.00 & 100.00 & 100.00 \\
\hline $\begin{array}{l}\text { MH, Ib/lbmole } \\
\text { HHV, Btu/lb mole } \\
\text { HHV, Btu/lb } \\
\text { HHV, Btu/SCF \& } 60 \text { deg F }\end{array}$ & $\begin{array}{r}23.127 \\
* 170,824.08 \\
* \quad 7,386.34 \\
\star \quad 450.25\end{array}$ & $\begin{array}{r}23.582 \\
17,830.25 \\
7,371.39 \\
458.17\end{array}$ & $\begin{array}{r}23.361 \\
174,553.95 \\
7.472 .04 \\
460.08\end{array}$ & $\begin{array}{r}23.222 \\
177.736 .95 \\
7.653 .77 \\
468.47\end{array}$ & $\begin{array}{r}23.787 \\
178,315.86 \\
7,496.37 \\
469.99\end{array}$ & $\begin{array}{r}23.389 \\
177,401.01 \\
7,584.82 \\
467.58\end{array}$ & $\begin{array}{r}23.711 \\
174,714.40 \\
7,368.60 \\
460.50\end{array}$ & $\begin{array}{r}23.055 \\
174.277 .93 \\
7.559 .36 \\
459.35\end{array}$ & $\begin{array}{r}23.413 \\
173,419.49 \\
7,406.92 \\
457.09\end{array}$ & $\begin{array}{r}23.216 \\
173,817.92 \\
7.487 .10 \\
458.14\end{array}$ \\
\hline $\begin{array}{l}c, \text { lb/lbmole } \\
c \text {, lb/lb gas } \\
c \text {, Ib/ScF } \\
\text { c, lb/lb maf wood } \\
\text { c Conv to Gas, } x\end{array}$ & $\begin{array}{r}10.1350 \\
* \quad 0.4382 \\
* \quad 0.0267 \\
* \quad 46.2396 \\
* \quad 46.737\end{array}$ & $\begin{array}{r}10.3644 \\
0.4395 \\
0.0273 \\
0.2994 \\
59.1438\end{array}$ & $\begin{array}{r}10.3368 \\
0.4425 \\
0.0272 \\
0.2245 \\
44.3299\end{array}$ & $\begin{array}{r}10.4509 \\
0.4500 \\
0.0275 \\
0.2953 \\
58.9572\end{array}$ & $\begin{array}{r}10.6959 \\
0.4497 \\
0.0282 \\
0.2904 \\
71.9350\end{array}$ & $\begin{array}{r}10.4845 \\
0.4483 \\
0.0276 \\
0.2841 \\
56.8910\end{array}$ & $\begin{array}{r}10.5001 \\
0.4428 \\
0.0277 \\
0.2826 \\
56.2359\end{array}$ & $\begin{array}{r}10.2287 \\
0.4437 \\
0.0270 \\
0.2707 \\
53.9345\end{array}$ & $\begin{array}{r}10.3380 \\
0.4415 \\
0.0272 \\
0.2719 \\
54.2292\end{array}$ & $\begin{array}{r}10.2587 \\
0.4419 \\
0.0270 \\
0.2636 \\
52.5732\end{array}$ \\
\hline $\begin{array}{l}\text { H, lb/lb mole } \\
H, \text { lb/lb gas } \\
H, \text { lb/scF } \\
H, \text { lb/lb maF nood } \\
H \text { Conv to Gas, wt } X\end{array}$ & 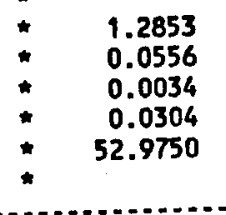 & $\begin{array}{r}1.3058 \\
0.0554 \\
0.0034 \\
0.0377 \\
63.8333\end{array}$ & $\begin{array}{r}1.3046 \\
0.0558 \\
0.0034 \\
0.0283 \\
46.0101\end{array}$ & $\begin{array}{r}1.2901 \\
0.0556 \\
0.0034 \\
0.0365 \\
60.2010\end{array}$ & $\begin{array}{r}1.2675 \\
0.0533 \\
0.0033 \\
0.0344 \\
54.8977\end{array}$ & $\begin{array}{r}1.2889 \\
0.0551 \\
0.0034 \\
0.0349 \\
54.0659\end{array}$ & $\begin{array}{r}1.2766 \\
0.0538 \\
0.0034 \\
0.0344 \\
64.2950\end{array}$ & $\begin{array}{r}1.2984 \\
0.0563 \\
0.0034 \\
0.0344 \\
53.7418\end{array}$ & $\begin{array}{r}1.2837 \\
0.0548 \\
0.0034 \\
0.0338 \\
55.4382\end{array}$ & $\begin{array}{r}1.3014 \\
0.0561 \\
0.0034 \\
0.0334 \\
54.9090\end{array}$ \\
\hline $\begin{array}{l}\text { Tar Estimate } \\
\text { Yield, Ib/lb maf feed } \\
\text { Eq Tar }=0.046-.00002^{\star} \text { Temp }\end{array}$ & 0.0167 & 0.0181 & 0.0176 & 0.0170 & 0.0178 & 0.0173 & 0.0186 & 0.0173 & 0.0185 & 0.0182 \\
\hline $\begin{array}{l}\text { Comp } \\
\text { C3H6, LB/lb maf wood } \\
\text { C6H6 } \\
\text { C10HB } \\
\text { C14H10 }\end{array}$ & $\begin{array}{l}* 0.004175 \\
\star \quad 0.004175 \\
\star \quad 0.004175 \\
* \quad 0.004175\end{array}$ & $\begin{array}{l}0.004525 \\
0.004525 \\
0.004525 \\
0.004525\end{array}$ & $\begin{array}{l}0.00439 \\
0.00439 \\
0.00439 \\
0.00439\end{array}$ & $\begin{array}{l}0.004245 \\
0.004245 \\
0.004245 \\
0.004245\end{array}$ & $\begin{array}{l}0.00446 \\
0.00446 \\
0.00446 \\
0.00446\end{array}$ & $\begin{array}{l}0.00432 \\
0.00432 \\
0.00432 \\
0.00432\end{array}$ & $\begin{array}{l}0.004645 \\
0.004645 \\
0.004645 \\
0.004645\end{array}$ & $\begin{array}{l}0.00432 \\
0.00432 \\
0.00432 \\
0.00432\end{array}$ & $\begin{array}{l}0.00463 \\
0.00463 \\
0.00463 \\
0.00463\end{array}$ & $\begin{array}{l}0.00456 \\
0.00456 \\
0.00456 \\
0.00456\end{array}$ \\
\hline $\begin{array}{l}\text { Ib } c / l b \text { maf wood } \\
\text { lb H/lb maf wood } \\
\text { lb } c / l b \text { dry wood } \\
\text { lb } H / l b \text { dry wood }\end{array}$ & $\begin{array}{l}0.0121 \\
* \quad 0.0046 \\
* \quad 0.0120 \\
* \quad 0.0046\end{array}$ & $\begin{array}{l}0.0131 \\
0.0050 \\
0.0130 \\
0.0050\end{array}$ & $\begin{array}{l}0.0127 \\
0.0049 \\
0.0126 \\
0.0049\end{array}$ & $\begin{array}{l}0.0123 \\
0.0047 \\
0.0121 \\
0.0047\end{array}$ & $\begin{array}{l}0.0129 \\
0.0050 \\
0.0128 \\
0.0049\end{array}$ & $\begin{array}{l}0.0125 \\
0.0048 \\
0.0124 \\
0.0048\end{array}$ & $\begin{array}{l}0.0134 \\
0.0052 \\
0.0133 \\
0.0051\end{array}$ & $\begin{array}{l}0.0125 \\
0.0048 \\
0.0124 \\
0.0048\end{array}$ & $\begin{array}{l}0.0134 \\
0.0051 \\
0.0133 \\
0.0051\end{array}$ & $\begin{array}{l}0.0132 \\
0.0051 \\
0.0131 \\
0.0050\end{array}$ \\
\hline
\end{tabular}




\begin{tabular}{|c|c|c|c|c|c|c|c|c|c|c|}
\hline Item/Test & 83 & 84 & 85 & 86 & 87 & 88 & 89 & 90 & 91 & 92 \\
\hline $\begin{array}{l}\text { Basis: } 1 \text { ib ory } \\
\text { Char est imate, Let } C=86, H=4 \text {, } \\
\text { lb Ash } \\
\text { lb C }\end{array}$ & $0=$ & 0.0050 & 0.0046 & 0.0091 & 0.0061 & 0.0061 & 0.0081 & 0.0099 & 0.0067 & 0.0067 \\
\hline $\begin{array}{l}\text { In } \\
\text { Out in Ges } \\
\text { out in Tar } \\
\text { Out in Char }\end{array}$ & $\begin{array}{l}0.5095 \\
\star \quad 0.2381 \\
\star \quad 0.0120 \\
\star \quad 0.2594\end{array}$ & $\begin{array}{l}0.5037 \\
0.2979 \\
0.0130 \\
0.1928\end{array}$ & $\begin{array}{l}0.5041 \\
0.2235 \\
0.0126 \\
0.2680\end{array}$ & $\begin{array}{l}0.4963 \\
0.2926 \\
0.0121 \\
0.1915\end{array}$ & $\begin{array}{l}0.4012 \\
0.2886 \\
0.0128 \\
0.0998\end{array}$ & $\begin{array}{l}0.4963 \\
0.2823 \\
0.0124 \\
0.2016\end{array}$ & $\begin{array}{l}0.4984 \\
0.2803 \\
0.0133 \\
0.2048\end{array}$ & $\begin{array}{l}0.4969 \\
0.2680 \\
0.0124 \\
0.2165\end{array}$ & $\begin{array}{l}0.4981 \\
0.2701 \\
0.0133 \\
0.2147\end{array}$ & $\begin{array}{l}0.4981 \\
0.2619 \\
0.0131 \\
0.2232\end{array}$ \\
\hline $\begin{array}{l}H \text { in char } \\
H \text { in gas } \\
H \text { in tar } \\
H \text { in } \\
H \text { in steam } \\
\text { Ib H2O }\end{array}$ & $\begin{array}{l}\text { * } \quad 0.0185 \\
\star \quad 0.0302 \\
\star \quad 0.0046 \\
\star \quad 0.1932 \\
\star \quad 0.1399 \\
\star \quad 1.2502\end{array}$ & $\begin{array}{l}0.0138 \\
0.0375 \\
0.0050 \\
0.1829 \\
0.1266 \\
1.1314\end{array}$ & $\begin{array}{l}0.0191 \\
0.0282 \\
0.0049 \\
0.2202 \\
0.1680 \\
1.5014\end{array}$ & $\begin{array}{l}0.0137 \\
0.0361 \\
0.0047 \\
0.1821 \\
0.1276 \\
1.1405\end{array}$ & $\begin{array}{l}0.0071 \\
0.0342 \\
0.0049 \\
0.1844 \\
0.1382 \\
1.2347\end{array}$ & $\begin{array}{l}0.0144 \\
0.0347 \\
0.0048 \\
0.1882 \\
0.1343 \\
1.2003\end{array}$ & $\begin{array}{l}0.0146 \\
0.0341 \\
0.0051 \\
0.2375 \\
0.1836 \\
1.6412\end{array}$ & $\begin{array}{l}0.0155 \\
0.0340 \\
0.0048 \\
0.1879 \\
0.1336 \\
1.1943\end{array}$ & $\begin{array}{l}0.0153 \\
0.0335 \\
0.0051 \\
0.2662 \\
0.2123 \\
1.8969\end{array}$ & $\begin{array}{l}0.0159 \\
0.0332 \\
0.0050 \\
0.2685 \\
0.2143 \\
1.9152\end{array}$ \\
\hline $\begin{array}{l}\text { Lb in } \\
\text { Dry Hood } \\
\text { Water }\end{array}$ & $\begin{array}{l}\text { * } \quad 1.0000 \\
* \quad 1.2175\end{array}$ & $\begin{array}{l}1.0000 \\
1.1092\end{array}$ & $\begin{array}{l}1.0000 \\
1.4202\end{array}$ & $\begin{array}{l}1.0000 \\
1.0911\end{array}$ & $\begin{array}{l}1.0000 \\
1.0914\end{array}$ & $\begin{array}{l}1.0000 \\
1.1080\end{array}$ & $\begin{array}{l}1.0000 \\
1.6486\end{array}$ & $\begin{array}{l}1.0000 \\
1.1134\end{array}$ & $\begin{array}{l}1.0000 \\
1.8387\end{array}$ & $\begin{array}{l}1.0000 \\
1.8589\end{array}$ \\
\hline $\begin{array}{l}\text { Lb Out } \\
\text { Gas } \\
\text { Tar } \\
\text { Char } \\
\text { H2O } \\
\text { Closure }\end{array}$ & $\begin{array}{r}0.5433 \\
0.0166 \\
* \quad 0.3115 \\
\quad 1.2502 \\
\quad 95.68\end{array}$ & $\begin{array}{r}0.6778 \\
0.0180 \\
.0 .2318 \\
1.1314 \\
97.62\end{array}$ & $\begin{array}{l}0.5050 \\
0.0175 \\
0.3199 \\
1.5014 \\
96.85\end{array}$ & $\begin{array}{l}0.6502 \\
0.0168 \\
0.2345 \\
1.1405 \\
97.65\end{array}$ & $\begin{array}{l}0.6418 \\
0.0177 \\
0.1235 \\
1.2347 \\
96.48\end{array}$ & $\begin{array}{l}0.6299 \\
0.0172 \\
0.2432 \\
1.2003 \\
99.17\end{array}$ & $\begin{array}{r}0.6329 \\
0.0184 \\
0.2491 \\
1.6412 \\
95.96\end{array}$ & $\begin{array}{l}0.6041 \\
0.0171 \\
0.2647 \\
1.1943 \\
98.43\end{array}$ & $\begin{array}{l}0.6117 \\
0.0184 \\
0.2593 \\
1.8969 \\
98.15\end{array}$ & $\begin{array}{r}0.5926 \\
0.0181 \\
0.2692 \\
1.9152 \\
97.77\end{array}$ \\
\hline $\begin{array}{l}\text { Water Conversion } X \\
\text { Water Conver., Ib/lb dry woodt }\end{array}$ & $\begin{array}{r}-2.69 \\
-0.0327\end{array}$ & $\begin{array}{r}-2.00 \\
-0.0222\end{array}$ & $\begin{array}{r}-5.72 \\
-0.0813\end{array}$ & $\begin{array}{r}-4.52 \\
-0.0493\end{array}$ & $\begin{array}{l}-13.14 \\
-0.1434\end{array}$ & $\begin{array}{r}-8.32 \\
-0.0922\end{array}$ & $\begin{array}{r}0.45 \\
0.0074\end{array}$ & $\begin{array}{r}-7.27 \\
-0.0809\end{array}$ & $\begin{array}{r}-3.16 \\
-0.0582\end{array}$ & $\begin{array}{r}-3.03 \\
-0.0563\end{array}$ \\
\hline
\end{tabular}




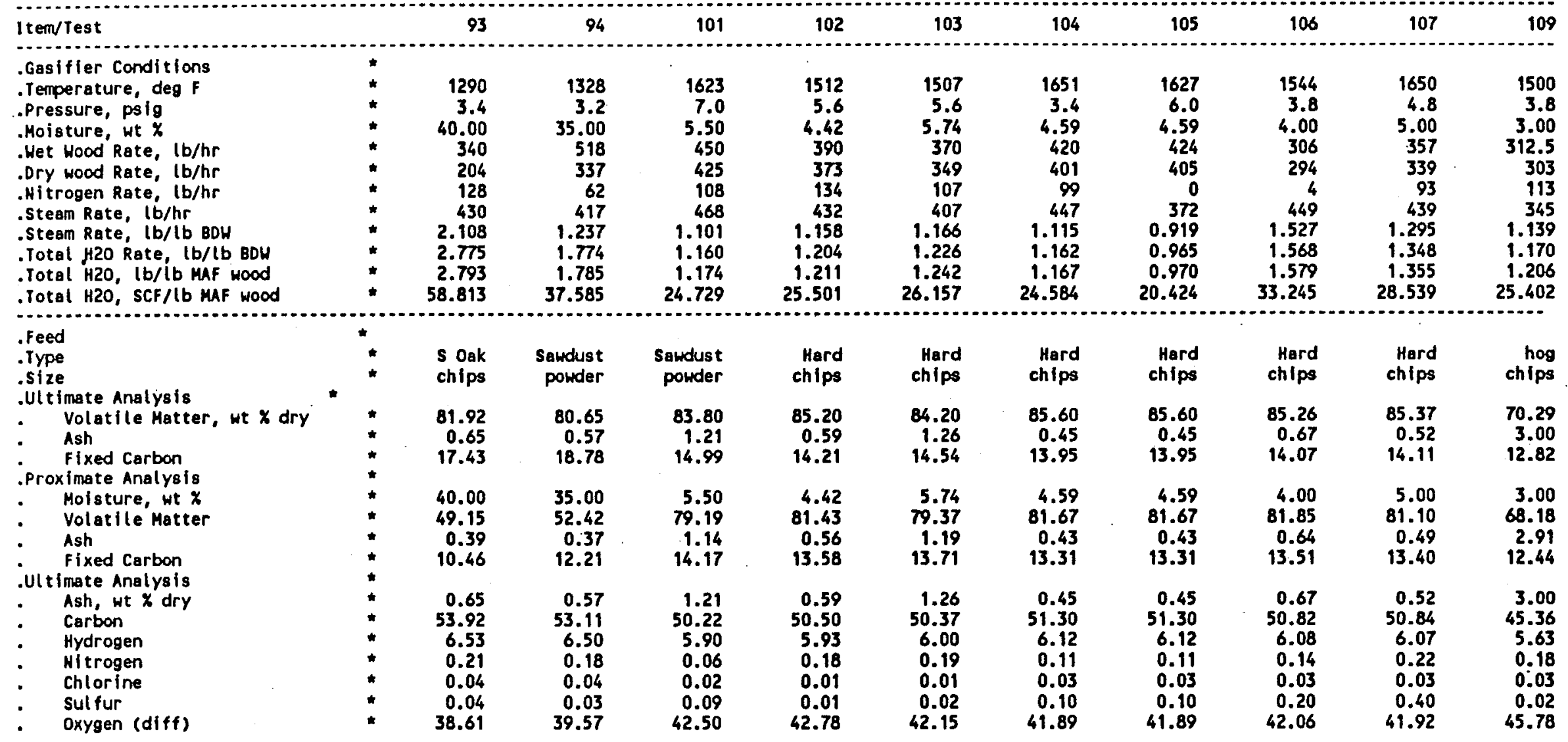




\begin{tabular}{|c|c|c|c|c|c|c|c|c|c|c|}
\hline .1tem/Test & 93 & $\begin{array}{c}94 \\
\ldots .\end{array}$ & 101 & 102 & 103 & 104 & 105 & 106 & 107 & 109 \\
\hline $\begin{array}{l}\text {-Product Gas } \\
\text { - Rate, SCF/lb MAF } \\
\text { - } \quad \text { Rate, SCF/ Ib BDW }\end{array}$ & $\begin{array}{l}8.82 \\
8.76\end{array}$ & $\begin{array}{l}6.31 \\
6.27\end{array}$ & $\begin{array}{l}14.45 \\
14.28\end{array}$ & $\begin{array}{l}12.02 \\
11.95\end{array}$ & $\begin{array}{l}11.78 \\
11.63\end{array}$ & $\begin{array}{l}13.83 \\
13.7\end{array}$ & $\begin{array}{l}14.57 \\
14.50\end{array}$ & $\begin{array}{l}12.99 \\
12.90\end{array}$ & $\begin{array}{l}14.93 \\
14.85\end{array}$ & $\begin{array}{l}9.96 \\
9.66\end{array}$ \\
\hline $\begin{array}{ll}: & \text { Dry H2 free Comp } \\
: & \mathrm{H2}, \text { mole } \mathrm{X} \\
: & \mathrm{CO} \\
: & \mathrm{CO} 2 \\
: & \mathrm{CH} 4 \\
: & \mathrm{C} 2 \mathrm{H} 2 \\
: & \mathrm{C} 2 \mathrm{H} 4 \\
: & \mathrm{C} 2 \mathrm{H} 6\end{array}$ & $\begin{array}{r}14.16 \\
51.75 \\
12.02 \\
16.26 \\
0.00 \\
4.74 \\
1.07\end{array}$ & $\begin{array}{r}16.81 \\
50.92 \\
10.53 \\
15.61 \\
0.25 \\
4.96 \\
0.92\end{array}$ & $\begin{array}{r}24.58 \\
40.75 \\
13.21 \\
16.75 \\
0.37 \\
4.06 \\
0.28\end{array}$ & $\begin{array}{r}21.90 \\
41.96 \\
13.99 \\
16.74 \\
0.29 \\
4.60 \\
0.52 \\
-\end{array}$ & $\begin{array}{l}20.87 \\
42.72 \\
14.02 \\
16.86 \\
0.35 \\
4.71 \\
0.47\end{array}$ & $\begin{array}{r}24.87 \\
41.05 \\
13.70 \\
15.61 \\
0.57 \\
4.09 \\
0.11 \\
0\end{array}$ & $\begin{array}{r}25.48 \\
40.21 \\
13.41 \\
16.45 \\
0.40 \\
3.84 \\
0.21\end{array}$ & $\begin{array}{l}22.47 \\
43.17 \\
12.54 \\
16.65 \\
0.34 \\
4.47 \\
0.36\end{array}$ & $\begin{array}{r}26.39 \\
41.09 \\
12.55 \\
15.65 \\
0.49 \\
3.69 \\
0.14\end{array}$ & $\begin{array}{r}21.54 \\
48.38 \\
9.07 \\
15.19 \\
0.27 \\
4.96 \\
0.59\end{array}$ \\
\hline & $\star \quad 100.00$ & 100.00 & 100.00 & 100.00 & 100.00 & 100.00 & 100.00 & 100.00 & 100.00 & 100.00 \\
\hline $\begin{array}{l}: \quad H H, \text { Ib/lbmole } \\
: \quad H H V, \text { Btu/lb mole } \\
: \quad H H V, \text { Btu/lb } \\
: \quad H H V, B t u / S C F \text { a } 60 \mathrm{deg} F \\
: \quad\end{array}$ & $\begin{array}{r}24.331 \\
* \quad 178,535.45 \\
* \quad 7.337 .81 \\
* \quad 470.57\end{array}$ & $\begin{array}{r}23.474 \\
180,020.76 \\
7,669.10 \\
474.49\end{array}$ & $\begin{array}{r}21.730 \\
172,478.48 \\
7,937.28 \\
454.61\end{array}$ & $\begin{array}{r}22.560 \\
175,057.09 \\
7,759.78 \\
461.41\end{array}$ & $\begin{array}{r}22.816 \\
175,843.09 \\
7.707 .15 \\
463.48\end{array}$ & $\begin{array}{r}21.862 \\
168,996.57 \\
7.730 .09 \\
445.43\end{array}$ & $\begin{array}{r}21.562 \\
170.142 .73 \\
7.890 .86 \\
448.45\end{array}$ & $\begin{array}{r}22.186 \\
175.303 .26 \\
7.901 .58 \\
462.05\end{array}$ & $\begin{array}{r}21.280 \\
168,393.20 \\
7,913.10 \\
443.84\end{array}$ & $\begin{array}{r}22.053 \\
179,033.91 \\
8,118.18 \\
471.89\end{array}$ \\
\hline $\begin{array}{l}: \quad c, \text { lb/lbmole } \\
: \quad \text {, lb/lb gas } \\
\text { c, Ib/ScF } \\
\text { : } \quad \text {, lb/lb MAF wood } \\
\text { c conv to Gas, } x\end{array}$ & $\begin{array}{rr}* & 11.0082 \\
\star \quad 0.4524 \\
\star \quad 0.0290 \\
\star \quad 0.2559 \\
* \quad 47.1527\end{array}$ & $\begin{array}{r}10.7284 \\
0.4570 \\
0.0283 \\
0.1784 \\
33.4046\end{array}$ & $\begin{array}{r}9.6245 \\
0.4429 \\
0.0254 \\
0.3666 \\
72.1085\end{array}$ & $\begin{array}{r}10.0305 \\
0.4446 \\
0.0264 \\
0.3178 \\
62.5560\end{array}$ & $\begin{array}{r}10.1686 \\
0.4457 \\
0.0268 \\
0.3157 \\
61.8916\end{array}$ & $\begin{array}{r}9.5969 \\
0.4390 \\
0.0253 \\
0.3498 \\
67.8860\end{array}$ & $\begin{array}{r}9.4852 \\
0.4399 \\
0.0250 \\
0.3643 \\
70.6859\end{array}$ & $\begin{array}{r}9.9332 \\
0.4477 \\
0.0262 \\
0.3401 \\
66.4734\end{array}$ & $\begin{array}{r}9.3603 \\
0.4399 \\
0.0247 \\
0.3683 \\
72.0746\end{array}$ & $\begin{array}{r}10.1230 \\
0.4590 \\
0.0267 \\
0.2657 \\
56.8290\end{array}$ \\
\hline $\begin{array}{l}H, \text { Ib/lb mole } \\
H, I b / l b \text { gas } \\
H_{1} \text { Ib/SCF } \\
H_{1} \text { Ib/lb MAF nood } \\
\text { H Conv to Gas, wt X }\end{array}$ & $\begin{array}{rr}* & 1.1968 \\
* & 0.0492 \\
* \quad 0.0032 \\
* \quad 0.0278 \\
* \quad 42.3292 \\
\end{array}$ & $\begin{array}{r}1.2288 \\
0.0523 \\
0.0032 \\
0.0204 \\
31.2628\end{array}$ & $\begin{array}{r}1.3589 \\
0.0625 \\
0.0036 \\
0.0518 \\
86.6569\end{array}$ & $\begin{array}{r}1.3391 \\
0.0594 \\
0.0035 \\
0.0424 \\
71.1204\end{array}$ & $\begin{array}{r}1.3258 \\
0.0581 \\
0.0035 \\
0.0412 \\
67.7431\end{array}$ & $\begin{array}{r}1.3137 \\
0.0601 \\
0.0035 \\
0.0479 \\
77.8950\end{array}$ & $\begin{array}{r}1.3524 \\
0.0627 \\
0.0036 \\
0.0519 \\
84.4807\end{array}$ & $\begin{array}{r}1.3330 \\
0.0601 \\
0.0035 \\
0.0456 \\
74.5649\end{array}$ & $\begin{array}{r}1.3300 \\
0.0625 \\
0.0035 \\
0.0523 \\
85.7767\end{array}$ & $\begin{array}{r}1.2877 \\
0.0584 \\
0.0034 \\
0.0338 \\
58.2422\end{array}$ \\
\hline $\begin{array}{l}\text { Tar Est imate } \\
\text { Yield, Ib/lb maf feed } \\
\text { Eq Tar=0.046-.00002*Temp }\end{array}$ & $\stackrel{\star}{\star}$ & 0.0194 & 0.0135 & 0.0158 & 0.0159 & 0.0130 & 0.0135 & 0.0151 & 0.0130 & 0.0160 \\
\hline $\begin{array}{ll}: & \text { Comp } \\
\text { C3H6, LB/lb maf HoOd } \\
\text { C6H6 } \\
: & \text { C10H8 } \\
\text { C14H1O }\end{array}$ & $\begin{array}{l}\star \quad 0.00505 \\
\star \quad 0.00505 \\
\star \quad 0.00505 \\
\star \quad 0.00505\end{array}$ & $\begin{array}{l}0.00486 \\
0.00486 \\
0.00486 \\
0.00486\end{array}$ & $\begin{array}{l}0.003385 \\
0.003385 \\
0.003385 \\
0.003385\end{array}$ & $\begin{array}{l}0.00394 \\
0.00394 \\
0.00394 \\
0.00394\end{array}$ & $\begin{array}{l}0.003965 \\
0.003965 \\
0.003965 \\
0.003965\end{array}$ & $\begin{array}{l}0.003245 \\
0.003245 \\
0.003245 \\
0.003245\end{array}$ & $\begin{array}{l}0.003365 \\
0.003365 \\
0.003365 \\
0.003365\end{array}$ & $\begin{array}{l}0.00378 \\
0.00378 \\
0.00378 \\
0.00378\end{array}$ & $\begin{array}{l}0.00325 \\
0.00325 \\
0.00325 \\
0.00325\end{array}$ & $\begin{array}{l}0.004 \\
0.004 \\
0.004 \\
0.004\end{array}$ \\
\hline $\begin{array}{l}\text { lb } \mathrm{c} / \mathrm{lb} \text { maf wood } \\
\text { lb } \mathrm{H} / \mathrm{lb} \text { maf wood } \\
\text { lb } \mathrm{c} / \mathrm{lb} \text { dry wood } \\
\text { lb } \mathrm{H} / \mathrm{lb} \text { dry wood }\end{array}$ & $\begin{array}{l}0.0146 \\
0.0056 \\
0.0145 \\
0.0056\end{array}$ & $\begin{array}{l}0.0140 \\
0.0054 \\
0.0140 \\
0.0054\end{array}$ & $\begin{array}{l}0.0098 \\
0.0038 \\
0.0097 \\
0.0037\end{array}$ & $\begin{array}{l}0.0114 \\
0.0044 \\
0.0113 \\
0.0044\end{array}$ & $\begin{array}{l}0.0115 \\
0.0044 \\
0.0113 \\
0.0044\end{array}$ & $\begin{array}{l}0.0094 \\
0.0036 \\
0.0093 \\
0.0036\end{array}$ & $\begin{array}{l}0.0097 \\
0.0037 \\
0.0097 \\
0.0037\end{array}$ & $\begin{array}{l}0.0109 \\
0.0042 \\
0.0108 \\
0.0042\end{array}$ & $\begin{array}{l}0.0094 \\
0.0036 \\
0.0093 \\
0.0036\end{array}$ & $\begin{array}{l}0.0116 \\
0.0044 \\
0.0112 \\
0.0043\end{array}$ \\
\hline
\end{tabular}




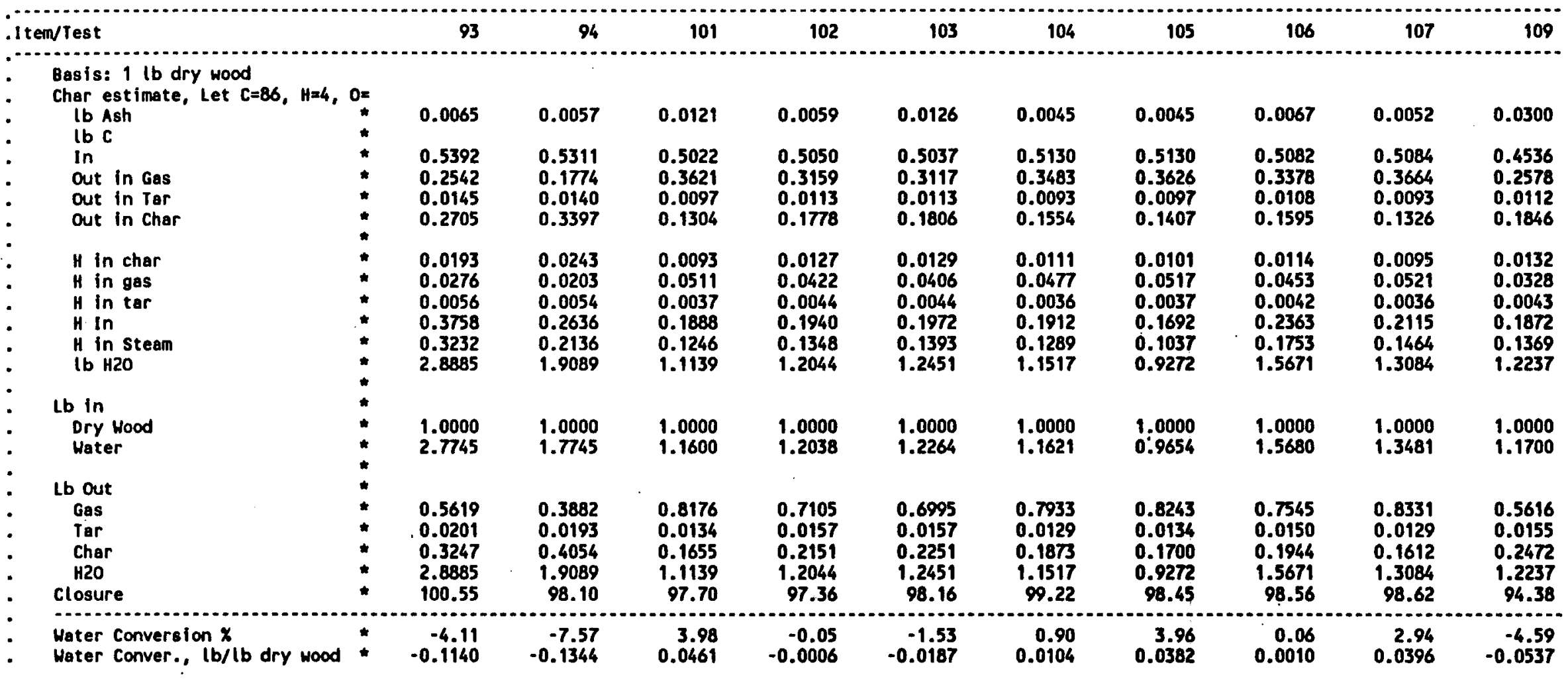




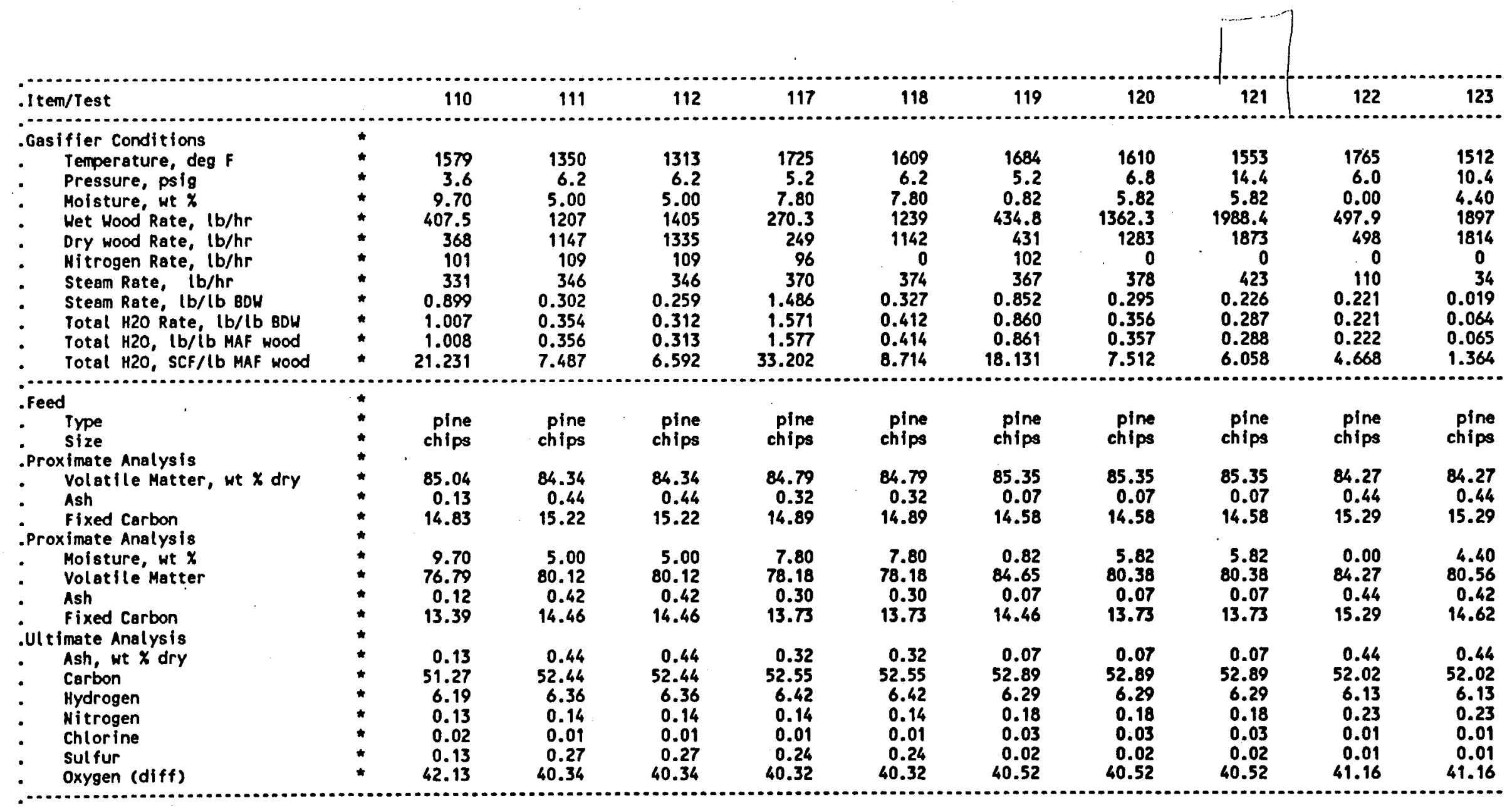




\begin{tabular}{|c|c|c|c|c|c|c|c|c|c|c|}
\hline .I tem/Test & 110 & 111 & 112 & 117 & 118 & 119 & 120 & 121 & 122 & 123 \\
\hline $\begin{array}{l}\text { Product Gas } \\
\text { - Rate, SCF/lb MAF } \\
\text { Rate, SCF/ Ib BDH }\end{array}$ & $\begin{array}{l}\star \quad 13.03 \\
\star \quad 13.01\end{array}$ & $\begin{array}{l}9.65 \\
9.61\end{array}$ & $\begin{array}{l}9.35 \\
9.31\end{array}$ & $\begin{array}{l}16.14 \\
16.09\end{array}$ & $\begin{array}{l}12.99 \\
12.95\end{array}$ & $\begin{array}{l}14.43 \\
14.42\end{array}$ & $\begin{array}{l}14.53 \\
14.52\end{array}$ & $\begin{array}{l}11.78 \\
11.77\end{array}$ & $\begin{array}{l}15.25 \\
15.18\end{array}$ & $\begin{array}{l}10.95 \\
10.90\end{array}$ \\
\hline 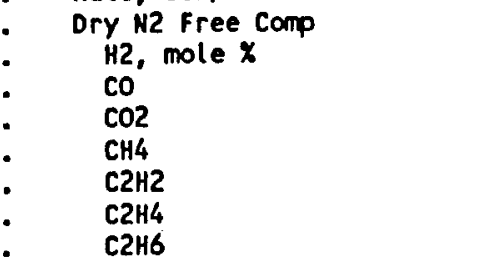 & $\begin{array}{r}22.99 \\
46.43 \\
9.62 \\
14.99 \\
0.41 \\
5.22 \\
0.34\end{array}$ & $\begin{array}{r}16.18 \\
49.46 \\
12.43 \\
16.05 \\
0.17 \\
4.60 \\
1.35\end{array}$ & $\begin{array}{r}15.20 \\
50.72 \\
12.30 \\
15.86 \\
0.18 \\
4.20 \\
1.54\end{array}$ & $\begin{array}{l}27.97 \\
43.29 \\
10.46 \\
14.12 \\
0.50 \\
3.66 \\
0.00\end{array}$ & $\begin{array}{r}20.96 \\
47.69 \\
9.30 \\
15.92 \\
0.26 \\
5.43 \\
0.44\end{array}$ & $\begin{array}{r}24.66 \\
46.67 \\
9.03 \\
14.85 \\
0.45 \\
4.18 \\
0.16\end{array}$ & $\begin{array}{r}20.23 \\
48.71 \\
9.16 \\
15.78 \\
0.23 \\
5.60 \\
0.53\end{array}$ & $\begin{array}{r}18.46 \\
49.41 \\
9.56 \\
16.24 \\
0.17 \\
5.30 \\
0.86\end{array}$ & $\begin{array}{r}26.96 \\
46.34 \\
8.50 \\
14.37 \\
0.34 \\
3.03 \\
0.46\end{array}$ & $\begin{array}{r}17.10 \\
50.63 \\
9.65 \\
16.48 \\
0.14 \\
4.84 \\
1.16\end{array}$ \\
\hline -ive & $\star \quad 100.00$ & 100.24 & 100.00 & 100.00 & 100.00 & 100.00 & 100.24 & 100.00 & 100.00 & 100.00 \\
\hline $\begin{array}{l}\text { HH, lb/lbmole } \\
\text { HHV, Btu/lb mole } \\
\text { HHV, Btu/lb } \\
\text { HHV, Btu/SCF a } 60 \mathrm{deg} F\end{array}$ & 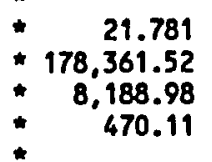 & $\begin{array}{r}23.966 \\
179.406 .51 \\
7.485 .85 \\
472.87\end{array}$ & $\begin{array}{r}24.159 \\
177,911.28 \\
7.364 .16 \\
468.93\end{array}$ & $\begin{array}{r}20.715 \\
166,089.73 \\
8,017.76 \\
437.77\end{array}$ & $\begin{array}{r}22.151 \\
182,065.71 \\
8,219.31 \\
479.88\end{array}$ & $\begin{array}{r}21.264 \\
172.877 .09 \\
8,130.04 \\
455.66\end{array}$ & $\begin{array}{r}22.405 \\
183,340.82 \\
8,183.09 \\
483.24\end{array}$ & $\begin{array}{r}22.814 \\
183,835.63 \\
8,057.84 \\
484.54\end{array}$ & $\begin{array}{r}20.647 \\
167,884.14 \\
8,131.32 \\
442.50\end{array}$ & $\begin{array}{r}23.160 \\
183,620.70 \\
7.928 .27 \\
483.98\end{array}$ \\
\hline 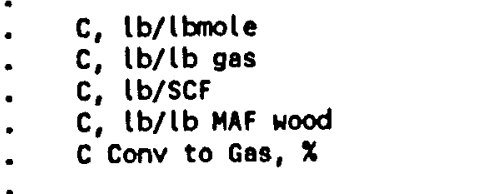 & $\begin{array}{r}\quad 9.9669 \\
* \quad 0.4576 \\
* \quad 0.0263 \\
* \quad 0.3423 \\
* \quad 66.6771\end{array}$ & $\begin{array}{r}10.8317 \\
0.4520 \\
0.0285 \\
0.2755 \\
52.3055\end{array}$ & $\begin{array}{r}10.8965 \\
0.4510 \\
0.0287 \\
0.2685 \\
50.9829\end{array}$ & $\begin{array}{l}9.1513 \\
0.4418 \\
0.0241 \\
0.3893 \\
73.8455\end{array}$ & $\begin{array}{r}10.2299 \\
0.4618 \\
0.0270 \\
0.3503 \\
66.4383\end{array}$ & $\begin{array}{l}9.6245 \\
0.4526 \\
0.0254 \\
0.3661 \\
69.1626\end{array}$ & $\begin{array}{r}10.3740 \\
0.4630 \\
0.0273 \\
0.3973 \\
75.0651\end{array}$ & $\begin{array}{r}10.5542 \\
0.4626 \\
0.0278 \\
0.3277 \\
61.9149\end{array}$ & $\begin{array}{r}9.2330 \\
0.4472 \\
0.0243 \\
0.3711 \\
71.0278\end{array}$ & $\begin{array}{r}10.6947 \\
0.4618 \\
0.0282 \\
0.3087 \\
59.0746\end{array}$ \\
\hline $\begin{array}{l}H, \text { Ib/lb mole } \\
H, \text { Ib/lb gas } \\
H, \text { Ib/SCF } \\
H, \text { Ib/lb MAF wood } \\
H \text { Conv to Gas, wt } X\end{array}$ & $\begin{array}{r}* \quad 1.3070 \\
* \quad 0.0600 \\
* \quad 0.0034 \\
* \quad 0.0449 \\
* \quad 72.4239\end{array}$ & $\begin{array}{r}1.2437 \\
0.0519 \\
0.0033 \\
0.0316 \\
49.5211\end{array}$ & $\begin{array}{r}1.2119 \\
0.0502 \\
0.0032 \\
0.0299 \\
46.7529\end{array}$ & $\begin{array}{r}1.2907 \\
0.0623 \\
0.0034 \\
0.0549 \\
85.2531\end{array}$ & $\begin{array}{r}1.3151 \\
0.0594 \\
0.0035 \\
0.0450 \\
69.9111\end{array}$ & $\begin{array}{l}1.2831 \\
0.0603 \\
0.0034 \\
0.0488 \\
77.5283\end{array}$ & $\begin{array}{l}1.3064 \\
0.0583 \\
0.0034 \\
0.0500 \\
79.4883\end{array}$ & $\begin{array}{r}1.2960 \\
0.0568 \\
0.0034 \\
0.0402 \\
63.9270\end{array}$ & $\begin{array}{r}1.2796 \\
0.0620 \\
0.0034 \\
0.0514 \\
83.5375\end{array}$ & $\begin{array}{r}1.2772 \\
0.0551 \\
0.0034 \\
0.0369 \\
59.8692\end{array}$ \\
\hline $\begin{array}{l}\text { Tar Est imate } \\
\text { Yield, Ib/lb maf feed } \\
\text { Eq Tar=0.046-.00002*Temp }\end{array}$ & $\star \quad 0.0144$ & 0.0190 & 0.0197 & 0.0115 & 0.0138 & 0.0123 & 0.0138 & 0.0149 & 0.0107 & 0.0158 \\
\hline $\begin{array}{l}\text { Comp } \\
\text { C3H6, L8/lb maf wood } \\
\text { C6H6 } \\
\text { C10H8 } \\
\text { C14H10 }\end{array}$ & $\begin{array}{l}\star \quad 0.003605 \\
\star \quad 0.003605 \\
\star \quad 0.003605 \\
\star \quad 0.003605\end{array}$ & $\begin{array}{l}0.00475 \\
0.00475 \\
0.00475 \\
0.00475\end{array}$ & $\begin{array}{l}0.004935 \\
0.004935 \\
0.004935 \\
0.004935\end{array}$ & $\begin{array}{l}0.002875 \\
0.002875 \\
0.002875 \\
0.002875\end{array}$ & $\begin{array}{l}0.003455 \\
0.003455 \\
0.003455 \\
0.003455\end{array}$ & $\begin{array}{l}0.00308 \\
0.00308 \\
0.00308 \\
0.00308\end{array}$ & $\begin{array}{l}0.00345 \\
0.00345 \\
0.00345 \\
0.00345\end{array}$ & $\begin{array}{l}0.003735 \\
0.003735 \\
0.003735 \\
0.003735\end{array}$ & $\begin{array}{l}0.002675 \\
0.002675 \\
0.002675 \\
0.002675\end{array}$ & $\begin{array}{l}0.00394 \\
0.00394 \\
0.00394 \\
0.00394\end{array}$ \\
\hline $\begin{array}{l}\text { lb } c / l b \text { maf wood } \\
\text { lb } H / l b \text { maf wood } \\
\text { lb } c / l b \text { dry wood } \\
\text { lb } H / l b \text { dry wood }\end{array}$ & $\begin{array}{r}* \quad 0.0104 \\
* \quad 0.0040 \\
+\quad 0.0104 \\
* \quad 0.0040\end{array}$ & $\begin{array}{l}0.0137 \\
0.0053 \\
0.0137 \\
0.0053\end{array}$ & $\begin{array}{l}0.0143 \\
0.0055 \\
0.0142 \\
0.0055\end{array}$ & $\begin{array}{l}0.0083 \\
0.0032 \\
0.0083 \\
0.0032\end{array}$ & $\begin{array}{l}0.0100 \\
0.0038 \\
0.0099 \\
0.0038\end{array}$ & $\begin{array}{l}0.0089 \\
0.0034 \\
0.0089 \\
0.0034\end{array}$ & $\begin{array}{l}0.0100 \\
0.0038 \\
0.0100 \\
0.0038\end{array}$ & $\begin{array}{l}0.0108 \\
0.0042 \\
0.0108 \\
0.0042\end{array}$ & $\begin{array}{l}0.0077 \\
0.0030 \\
0.0077 \\
0.0030\end{array}$ & $\begin{array}{l}0.0114 \\
0.0044 \\
0.0113 \\
0.0044\end{array}$ \\
\hline
\end{tabular}




\begin{tabular}{|c|c|c|c|c|c|c|c|c|c|c|}
\hline Item/Test & 110 & 111 & 112 & 117 & 118 & 119 & 120 & 121 & 122 & 123 \\
\hline $\begin{array}{l}\text { Basis: } 1 \text { lb dry wood } \\
\text { - Char est imate, Let } \mathrm{C}=86, \mathrm{H}=4, \mathrm{O} \\
\text { Ib Ash } \\
\text { Ib C }\end{array}$ & 0.0013 & 0.0044 & 0.0044 & 0.0032 & 0.0032 & 0.0007 & 0.0007 & 0.0007 & 0.0044 & 0.0044 \\
\hline $\begin{array}{ll} & \text { Ib C } \\
\text { In } & \text { Out in Gas } \\
\text { Out in Tar } \\
\text { Out in Char }\end{array}$ & $\begin{array}{l}0.5127 \\
* \quad 0.3419 \\
* \quad 0.0104 \\
* \quad 0.1604\end{array}$ & $\begin{array}{l}0.5244 \\
0.2743 \\
0.0137 \\
0.2365\end{array}$ & $\begin{array}{l}0.5244 \\
0.2674 \\
0.0142 \\
0.2429\end{array}$ & $\begin{array}{l}0.5255 \\
0.3881 \\
0.0083 \\
0.1292\end{array}$ & $\begin{array}{l}0.5255 \\
0.3491 \\
0.0099 \\
0.1664\end{array}$ & $\begin{array}{l}0.5289 \\
0.3658 \\
0.0089 \\
0.1542\end{array}$ & $\begin{array}{l}0.5289 \\
0.3970 \\
0.0100 \\
0.1219\end{array}$ & $\begin{array}{l}0.5289 \\
0.3275 \\
0.0108 \\
0.1907\end{array}$ & $\begin{array}{l}0.5202 \\
0.3695 \\
0.0077 \\
0.1430\end{array}$ & $\begin{array}{l}0.5202 \\
0.3073 \\
0.0113 \\
0.2016\end{array}$ \\
\hline $\begin{array}{ll}: & H \text { in char } \\
: & H \text { in gas } \\
- & H \text { in tar } \\
\cdot & H \text { in } \\
: & H \text { in steam } \\
\because \quad & \text { Ib } H 20 \\
-\quad & \end{array}$ & $\begin{array}{ll}* & 0.0115 \\
* & 0.0448 \\
* & 0.0040 \\
* & 0.1746 \\
* \quad 0.1143 \\
* \quad 1.0211\end{array}$ & $\begin{array}{l}0.0169 \\
0.0315 \\
0.0053 \\
0.1032 \\
0.0496 \\
0.4429\end{array}$ & $\begin{array}{l}0.0173 \\
0.0297 \\
0.0055 \\
0.0985 \\
0.0459 \\
0.4104\end{array}$ & $\begin{array}{l}0.0092 \\
0.0547 \\
0.0032 \\
0.2400 \\
0.1729 \\
1.5452\end{array}$ & $\begin{array}{l}0.0119 \\
0.0449 \\
0.0038 \\
0.1103 \\
0.0497 \\
0.4446\end{array}$ & $\begin{array}{l}0.0110 \\
0.0488 \\
0.0034 \\
0.1592 \\
0.0960 \\
0.8576\end{array}$ & $\begin{array}{l}0.0087 \\
0.0500 \\
0.0038 \\
0.1028 \\
0.0402 \\
0.3596\end{array}$ & $\begin{array}{l}0.0136 \\
0.0402 \\
0.0042 \\
0.0951 \\
0.0371 \\
0.3314\end{array}$ & $\begin{array}{l}0.0102 \\
0.0512 \\
0.0030 \\
0.0860 \\
0.0216 \\
0.1931\end{array}$ & $\begin{array}{l}0.0144 \\
0.0367 \\
0.0044 \\
0.0685 \\
0.0131 \\
0.1167\end{array}$ \\
\hline $\begin{array}{l}\text { Lb in } \\
\text { Dry Wood } \\
\text { Hater }\end{array}$ & $\begin{array}{l}\star \\
\star \quad 1.0000 \\
\star \quad 1.0068\end{array}$ & $\begin{array}{l}1.0000 \\
0.3540\end{array}$ & $\begin{array}{l}1.0000 \\
0.3116\end{array}$ & $\begin{array}{l}1.0000 \\
1.5715\end{array}$ & $\begin{array}{l}1.0000 \\
0.4124\end{array}$ & $\begin{array}{l}1.0000 \\
0.8603\end{array}$ & $\begin{array}{l}1.0000 \\
0.3564\end{array}$ & $\begin{array}{l}1.0000 \\
0.2875\end{array}$ & $\begin{array}{l}1.0000 \\
0.2207\end{array}$ & $\begin{array}{l}1.0000 \\
0.0645\end{array}$ \\
\hline $\begin{array}{ll}: & \text { Lb Out } \\
\text { Gas } \\
: & \text { Tar } \\
\text { Char } \\
: & \text { H2O } \\
: & \text { Closure }\end{array}$ & $\begin{array}{r}0.7471 \\
* \quad 0.0144 \\
* \quad 0.1901 \\
* \quad 1.0211 \\
* \quad 98.30 \\
\end{array}$ & $\begin{array}{r}0.6069 \\
0.0189 \\
0.2826 \\
0.4429 \\
99.81\end{array}$ & $\begin{array}{l}0.5928 \\
0.0197 \\
0.2901 \\
0.4104 \\
100.10\end{array}$ & $\begin{array}{l}0.8784 \\
0.0115 \\
0.1552 \\
1.5452 \\
100.73\end{array}$ & $\begin{array}{l}0.7560 \\
0.0138 \\
0.1990 \\
0.4446 \\
100.07\end{array}$ & $\begin{array}{l}0.8082 \\
0.0123 \\
0.1821 \\
0.8576 \\
100.00\end{array}$ & $\begin{array}{l}0.8574 \\
0.0138 \\
0.1441 \\
0.3596 \\
101.37\end{array}$ & $\begin{array}{r}0.7079 \\
0.0149 \\
0.2250 \\
0.3314 \\
99.36\end{array}$ & $\begin{array}{l}0.8262 \\
0.0107 \\
0.1727 \\
0.1931 \\
98.52\end{array}$ & $\begin{array}{r}0.6655 \\
0.0157 \\
0.2415 \\
0.1167 \\
97.64\end{array}$ \\
\hline $\begin{array}{l}\text { Noter Conversion } x \\
\text { Nater Conver., }(\mathrm{b} / \mathrm{lb} \text { dry wood }\end{array}$ & $\begin{array}{r}-1.42 \\
* \quad-0.0143\end{array}$ & $\begin{array}{r}-25.14 \\
-0.0890\end{array}$ & $\begin{array}{r}-31.70 \\
-0.0988\end{array}$ & $\begin{array}{r}1.68 \\
0.0263\end{array}$ & $\begin{array}{r}-7.80 \\
-0.0322\end{array}$ & $\begin{array}{r}0.32 \\
0.0027\end{array}$ & $\begin{array}{r}-0.90 \\
-0.0032\end{array}$ & $\begin{array}{r}-15.30 \\
-0.0440\end{array}$ & $\begin{array}{r}12.50 \\
0.0276\end{array}$ & $\begin{array}{r}-80.92 \\
-0.0522\end{array}$ \\
\hline
\end{tabular}




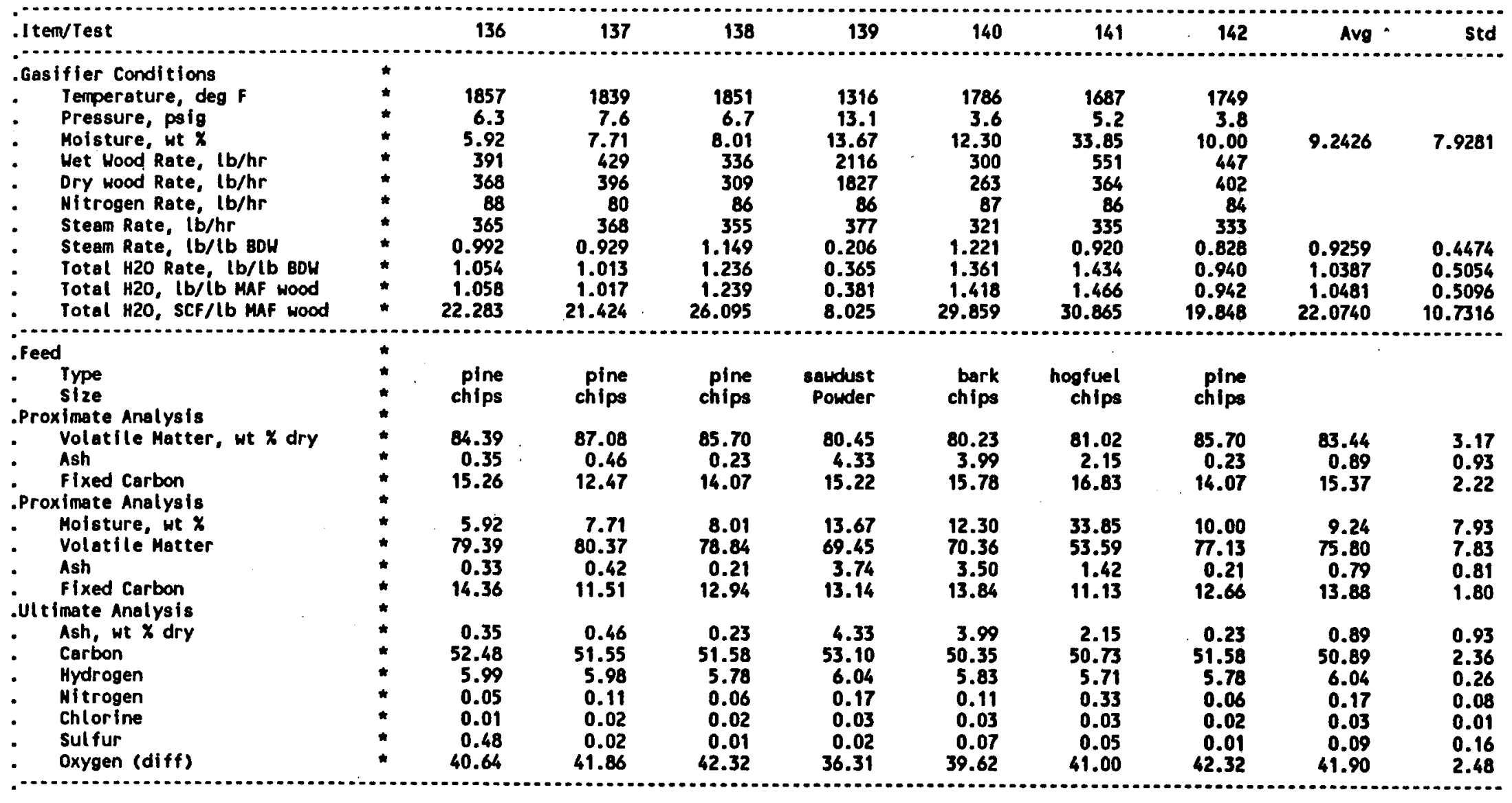




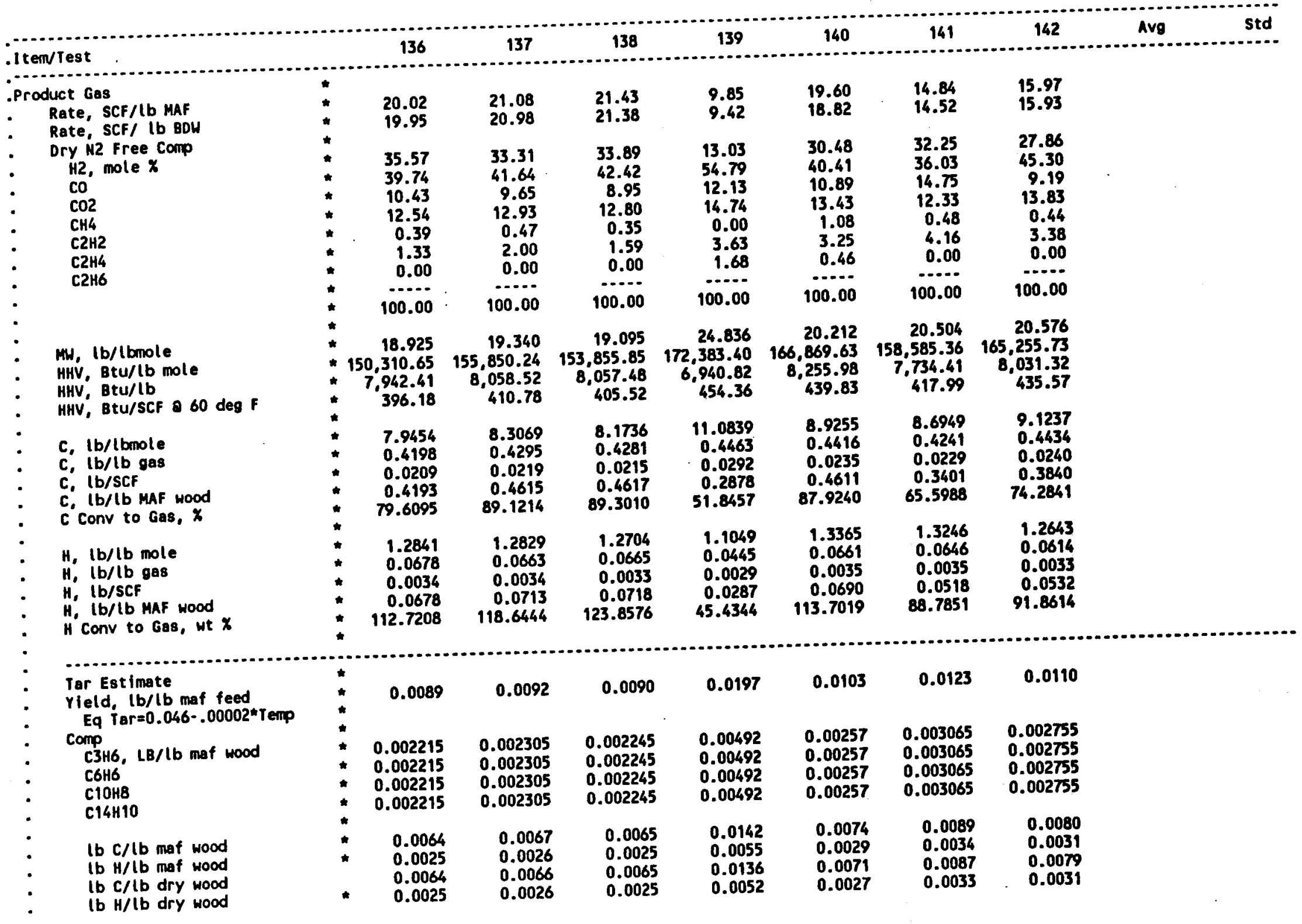




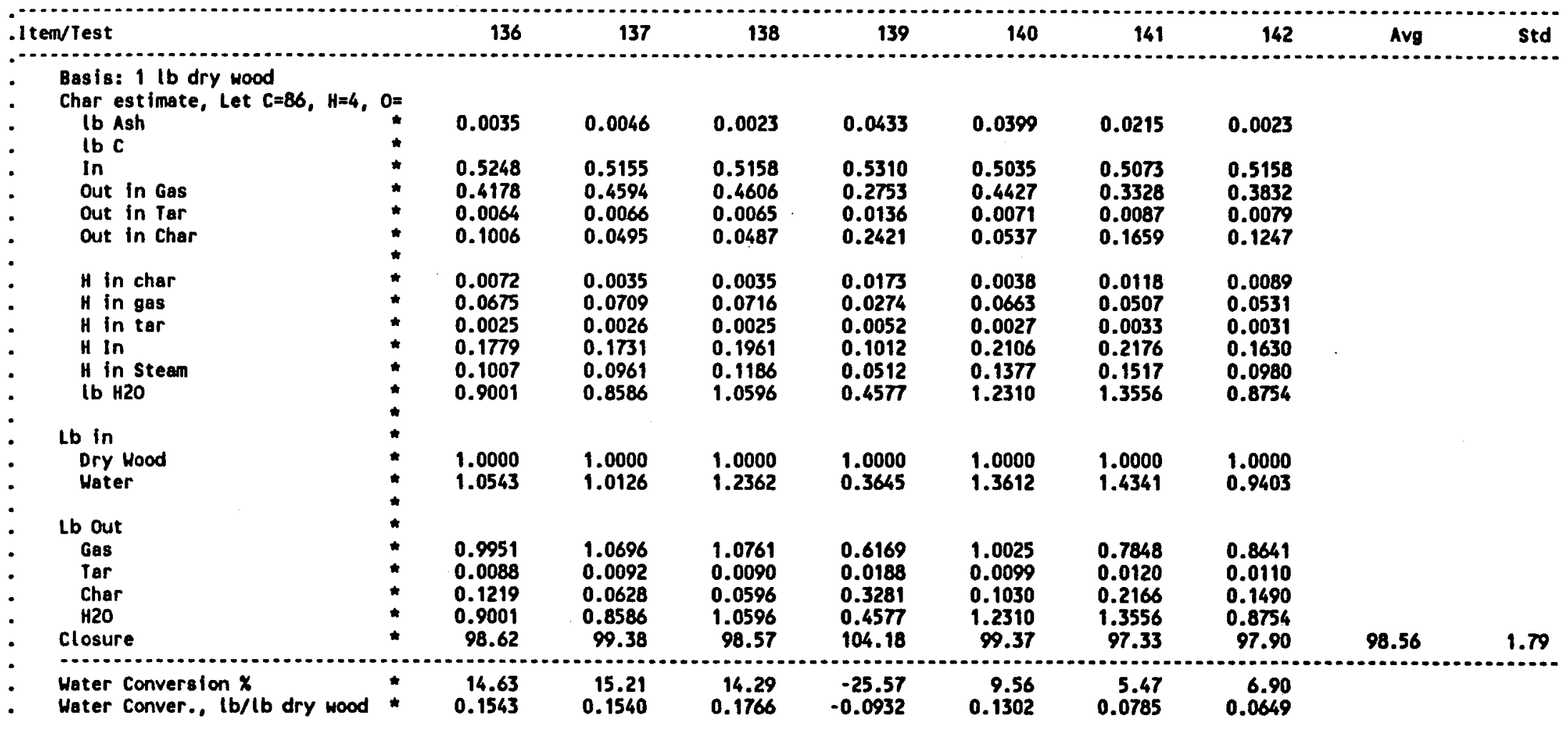


APPENDIX 8 


\section{BCL LEAST SQUARES FITS}

1. Dry Gas Yield, ScF/maF tb Regression Output:

$\begin{array}{ll}\text { Constant } & \\ \text { Std Err of Y Est } \\ R \text { Squared } \\ \text { No. of Observations } \\ \text { Degrees of Freedom } \\ \text { X Coefficient(s) } & -0.04332509 \\ \text { Std Err of Coef. } & 0.020685743 \\ \text { t Value } & -2.09446215 \\ & 2.015061631 \\ \text { t Probability } & 0.021949226 \\ & \\ \text { F Value } & 257.7194211 \\ & 0.111111111 \\ & 0.006535948 \\ & 8.838240047 \\ \text { F Probability } & 1.4827 E-17\end{array}$

28.99260129

0.947115477

0.938118681

37

0.000020966

$6.5312 E-06$

3.210111277

2.969644219

0.0014916

2. Gas Yield, lb/lb dry wood

\begin{tabular}{|c|c|}
\hline $\begin{array}{l}\text { Constant } \\
\text { Std Err of Y Est } \\
R \text { Squared } \\
\text { No. of Observations } \\
\text { Degrees of Freedom }\end{array}$ & 0 \\
\hline $\begin{array}{l}x \text { Coefficient(s) } \\
\text { std Err of Coef. }\end{array}$ & $\begin{array}{l}-0.00022057 \\
0.001289922\end{array}$ \\
\hline $\begin{array}{l}\text { t Value } \\
\text { t Probability }\end{array}$ & $\begin{array}{l}-0.1709956 \\
0.169701795 \\
0.432622395\end{array}$ \\
\hline f Probability & $\begin{array}{c}115.8339428 \\
0.111111111 \\
0.006535948 \\
7.641883919 \\
4.3112 E-14\end{array}$ \\
\hline
\end{tabular}

3. Tar Yield, lb/lb dry wood

Regression Output:

Constant

.155529322

0.059060263

0.872020662

37

$.76972-07$

4.0728E-07

Std Err of Y Est

$R$ Squared

0.923630659

0.91114971

No. of Observations

Degrees of Freedom

0.045494068

0.000118381

0.9985358

35
$X$ Coefficient(s)
Std Err of Coef.
$-1.9759 E-05$
$1.2789 E-07$
t Value
$-154.495416$
t Probability
8.29468485
$5.6747 \mathrm{E}-16$ 
TABLE 7 (CONT.)

4. Char Yield, lb/tb dry wood Regression Output:

Constant

Std Err of Y Est

$R$ squared

No. of Observations

Degrees of Freedom

0.755025269

0.046192661

0.654807013

$X$ Coefficient(s)

std Err of Coef.

$-0.00030212-3.1178 E-08$

t Value

$0.0010088843 .1854 E-07$

t Probability

$-0.29946257-0.0978786$

0.297064820 .097152064

F value $\quad 32.26781398$

F velue $\quad 0.111111111$

0.006535948

5.388529103

F Probability

3.8988E-08

5. H2, mole $X$

\begin{tabular}{|c|c|c|}
\hline $\begin{array}{l}\text { Constant } \\
\text { Std Err of Y Est } \\
R \text { Squared } \\
\text { No. of Observations } \\
\text { Degrees of Freedom }\end{array}$ & Dutput: & $\begin{array}{r}17.9961 \\
1.5945 \\
0.9178 \\
37.0000 \\
34.0000\end{array}$ \\
\hline $\begin{array}{l}\text { X Coefficient(s) } \\
\text { std Err of Coef. }\end{array}$ & $\begin{array}{c}-0.02644794 \\
0.034824791\end{array}$ & $\begin{array}{l}0.00001893 \\
0.000010995\end{array}$ \\
\hline $\begin{array}{l}\text { Value } \\
\text { Probability }\end{array}$ & $\begin{array}{l}-0.7594591 \\
0.750697843 \\
0.226417374\end{array}$ & $\begin{array}{l}1.721579457 \\
1.67285326 \\
0.047178091\end{array}$ \\
\hline F Value & $\begin{array}{l}189.8141747 \\
0.111111111 \\
0.006535948 \\
8.407872216 \\
2.6612 E-16\end{array}$ & \\
\hline
\end{tabular}

6. $\infty$, wole $x$

Regression output:

Constant

Std Err of $Y$ Est

$R$ Squared

No. of Observations

Degrees of Freedom

133.4594

2.4738

0.3974

37.0000

34.0000

$x$ Coefficient(s) std Err of Coef.

$\begin{array}{lll}-0.10290149 & 0.000028792\end{array}$ 0.0540296270 .000017059

t Value

t Probability

$-1.9045382 \quad 1.687769178$

1.8420432311 .641333522

F Value

0.0327345070 .050364078

F Probability

11.21026844

0.111111111

0.006535948

3.51363458

0.000221109 
7. $\cos$, mole $\%$

Regression output:

Constant

Std Err of Y Est

$-9.5251$

$R$ squared

1.8040

No. of Observations

0.4974

Degrees of Freedom

37.0000

$X$ Coefficient(s)

34.0000

Std Err of Coef.

$0.037888569-1.4927 E-05$

t value

t Probability 0.0394016340 .000012441

F value

$0.961598939-1.19987957$

$0.948103907 \quad 1.178645015$

0.1715383760 .119269716

12.17966128

0.1111111111

0.006535948

F Probebility

3.654061653

0.000129184

8. CH4, mole $x$

Constant

Regression output:

Std Err of Y Est

$-13.8203$

$R$ squared

No. of Observations

0.7822

0.6933

Degrees of Freedom

$X$ Coefficient(s)

34.0000

std Err of Coef.

$0.044178788-1.6167 E-05$

t Value

$2.585853318-2.99706 \% 96$

t Probability

$2.469242287 \quad 2.796085158$

f Value

0.0071578190 .002586159

38.43244924

0.111111111

0.006535948

5.708786026

6.6430E-09

9. C2H2, wole $x$

$\begin{array}{ll}\text { Constant } & \\ \text { Std Err of Y Est } & \\ \text { R Squared } & \\ \text { No. of Observations } \\ \text { Degrees of Freedom } \\ \\ \text { X Coefficient(s) } & 0.005449933 \\ \text { Std Err of Coef. } & 0.001564876 \\ & \\ \text { t Value } & 3.482660609 \\ \text { t Probability } & 2.992308924 \\ & 0.001384292 \\ \text { F Value } & 24.02371458 \\ & 0.111111111 \\ & 0.011695906 \\ & 4.291022394 \\ \text { F Probability } & 8.97286-06\end{array}$

$-4.31140373$

0.056156337

0.716618516

22

19

$1.5610 E-06$

4.8968E-07

$-3.18792205$

2.794416185

0.002599547

$8.9728 E-06$ 
10. C2H4, mole $x$

Regression Output:

Constant

Std Err of $Y$ Est

$R$ Squared

Mo. of Observations

Degrees of Freedom

$-38.2576352$

0.322371315

0.322371315
0.876167713

$x$ Coefficient(s)

Std Err of Coef.

$0.058434712-1.98685-05$

t Value

$0.007040842 \quad 2.2231 E-06$

t Probability

$8.299392915-8.93736877$

f Value

$5.806651565 \quad 6.01602073$

3.8117E-09 1.1365E-09

0.111111111

0.006535948

F Probability

7.703145134

2.8817E-14

11. C2H6, mole $x$

\begin{tabular}{lrr}
\multicolumn{2}{c}{ Regression Output: } & 11.11368612 \\
Constant & & 0.134430177 \\
Std Err of Y Est & & 0.852017036 \\
R Squared & 37 \\
No. of Observations & 34 \\
Degrees of Freedom & & \\
& & \\
$X$ Coefficient(s) & -0.01166677 & $3.0640 E-06$ \\
Std Err of Coef. & 0.00293606 & $9.2702 E-07$ \\
& & \\
t Value & -3.97361304 & 3.305165152 \\
& 3.553365071 & 3.04535283 \\
t Probability & 0.000190275 & 0.001161968 \\
& & \\
F Value & 97.87808856 \\
& 0.111111111 & \\
& 0.006535948 & \\
F Probability & 7.363029758 \\
\end{tabular}

12. H20 Conv, $x$

$\begin{array}{lrr}\text { Constant Regression Output: } & 0.289594752 \\ \text { Std Err of Y Est } & & 0.032742674 \\ \text { R Squared } & 0.841575638 \\ \text { No. of Observations } & & 47 \\ \text { Degrees of Freedom } & & 44 \\ & & \\ \text { X Coefficient(s) } & -8.9048 E-04 & 4.3384 E-07 \\ \text { Std Err of Coef. } & 5.8863 E-04 & 1.8809 E-07 \\ \text { t Velue } & -1.5128183 & 2.306574776 \\ & 1.485035998 & 2.227133353 \\ \text { t Probability } & 0.068767037 & 0.012969253 \\ & & \\ \text { F Value } & 116.8675309 & \\ & 0.111111111 & \\ & 0.005050505 & \\ \text { F Probability } & 8.237266254 & \end{array}$


APPENOIX 9 
Feed

Proximate Analysis

Volatile Matter, wt $x d r y$

83.44

Ash

Fixed Carbon

0.89

Proximate Analysis

Moisture, ut $X$

Volatile Matter

15.37

Ash

9.24

75.80

0.79

Fixed Carbon

Ut timate Analysis

Ash, wt $X$ dry

Carbon

Hydrogen

Nitrogen

Chlorine

sulfur

Oxygen (diff)

13.88

Dry

0.89

50.89

6.04

0.17

0.03

0.09

MA

Hiv, Btu/lb

IGT

Boie

41.90

51.35

New

8,726

8,539

8,608

Total Hater, Ib/lb dry wood

0.500

9.243
0.102

Feed Moisture, $x$

Feed Moisture, lb/lb dry wood

Stean, lb/lb dry wood

0.398

Total feed, lb/lb dry wood

1.500

Plant size, dTPD

2000

Dry wood rate, Ib/hr

$166,666.667$

Moisture, Ib/hr

$16,972.993$

Steem, Ib/hr

$66,360.340$

Total, Ib/hr

$250,000.000$

Variable

HZO Conversion tb/lb ory wood SCF/lb MiF uood lb/lb dry wood tb/lb dry wood

Gas

Tar

Char

H2

Ib/ln dry wood

mole $x$

mole $x$

mole $x$

$\cos$

mole $x$

mole $x$

mole $x$

C2H4

mole $\mathbf{x}$

$\wedge$

6.10

0.17

0.03

0.09

42.28

C2H6

2.8959E-01 -8.9048E-04 4.3384E-07

2.8993E+01 -4.3325E-02 2.0966E-05

1.5553E-01 -2.2057E-04 3.7617E-07

4.5494E-02 -1.9759E-05

7.5503E-01 -3.0212E-04 -3.2178E-08

$1.7996 E+01-2.6448 E-02 \quad 1.8930 E-05$

$1.3346 E+02-1.0290 E-01 \quad 2.8792 E-05$

$-9.5251 E+00 \quad 3.7889 E-02-1.4927 E-05$

$-1.3820 E+01$ 4.4179E-02 -1.6167E-05

$-4.3114 E+00 \quad 5.4499 E-03-1.5610 E-06$

$-3.8258 E+01 \quad 5.8435 E-02-1.9868 E-05$

$1.1114 E+01-1.1667 E-02 \quad 3.0640 E-06$

$$
x=A+B T+C T^{2}
$$

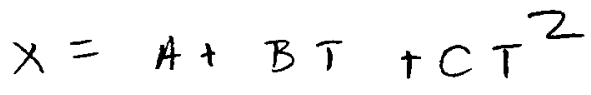


APPENDIX 10

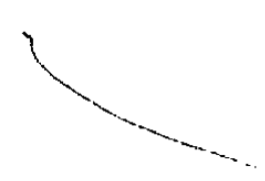




H2, Ib/lb total feed
CO
CO2
CH4
C2H2
C2H4
C2H6
C3H6
C6H6
C1OH8
C14H10
H2O
Ash
Char, MAF
TOtal
H2, moles/lb feed
CO
CO2
CH4
C2H2
C2H4
C2H6
C3H6
C6H6
C1OH8
C14H1O
H2O

H2, wole $x$
Co
CO2
CH4
C2H2
C2H4
C2H6
C3H6
C6H6
C1OH8
C14H1O
H2O

ASPEN IMPUT FILE: BCL RECYCLE CASE

$\begin{array}{lllll}1300 & 1325 & 1350 & 1375 & 1400\end{array}$

\begin{tabular}{|c|c|c|c|c|}
\hline $\begin{array}{l}4.3388 E-03 \\
1.8679 E-01 \\
8.8040 E-02 \\
3.6046 E-02 \\
4.8608 E-04 \\
1.5982 E-02 \\
4.6650 E-03 \\
3.3012 E-03 \\
3.3012 E-03 \\
3.3012 E-03 \\
3.3012 E-03 \\
4.4407 E-01 \\
5.9191 E-03 \\
2.0046 E-01 \\
1.0000 E+00\end{array}$ & $\begin{array}{l}4.7025 E-03 \\
1.9242 E-01 \\
9.1789 E-02 \\
3.7766 E-02 \\
6.3477 E-04 \\
1.7335 E-02 \\
4.4820 E-03 \\
3.2189 E-03 \\
3.2189 E-03 \\
3.2189 E-03 \\
3.2189 E-03 \\
4.3801 E-01 \\
5.9191 E-03 \\
1.9406 E-01 \\
1.0000 E+00\end{array}$ & $\begin{array}{l}5.0966 E-03 \\
1.9830 E-01 \\
9.5561 E-02 \\
3.9513 E-02 \\
7.8808 E-04 \\
1.8669 E-02 \\
4.2899 E-03 \\
3.1366 E-03 \\
3.1366 E-03 \\
3.1366 E-03 \\
3.1366 E-03 \\
4.3167 E-01 \\
5.9191 E-03 \\
1.8764 E-01 \\
1.0000 E+00\end{array}$ & $\begin{array}{l}5.5234 E-03 \\
2.0447 E-01 \\
9.9345 E-02 \\
4.1285 E-02 \\
9.4574 E-04 \\
1.9976 E-02 \\
4.0892 E-03 \\
3.0542 E-03 \\
3.0542 E-03 \\
3.0542 E-03 \\
3.0542 E-03 \\
4.2505 E-01 \\
5.9191 E-03 \\
1.8119 E-01 \\
1.0000 E+00\end{array}$ & $\begin{array}{l}5.9853 E-03 \\
2.1092 E-01 \\
1.0313 E-01 \\
4.3078 E-02 \\
1.1074 E-03 \\
2.1246 E-02 \\
3.8805 E-03 \\
2.9719 E-03 \\
2.9719 E-03 \\
2.9719 E-03 \\
2.9719 E-03 \\
4.1813 E-01 \\
5.9191 E-03 \\
1.7471 E-01 \\
1.0000 E+00\end{array}$ \\
\hline $\begin{array}{l}2.1526 E-03 \\
6.6684 E-03 \\
2.0005 E-03 \\
2.2669 E-03 \\
1.8668 E-05 \\
5.6969 E-04 \\
1.5517 E-04 \\
8.4523 E-05\end{array}$ & $\begin{array}{l}2.3328 E-03 \\
6.8695 E-03 \\
2.0856 E-03 \\
2.3541 E-03 \\
2.4378 E-05 \\
6.1790 E-04 \\
1.4908 E-04 \\
8.2415 E-05 \\
4.1207 E-05 \\
3.0319 E-05 \\
1.8060 E-05\end{array}$ & $\begin{array}{l}2.5283 E-03 \\
7.0796 E-03 \\
2.1713 E-03 \\
2.4630 E-03 \\
3.0266 E-05 \\
6.6547 E-04 \\
1.4269 E-04 \\
8.0307 E-05 \\
4.0153 E-05 \\
2.9543 E-05 \\
1.7598 E-05\end{array}$ & $\begin{array}{l}2.7400 E-03 \\
7.2996 E-03 \\
2.2573 E-03 \\
2.5734 E-03 \\
3.6321 E-05 \\
7.1207 E-04 \\
1.3602 E-04 \\
7.8199 E-05 \\
3.9099 E-05 \\
2.8768 E-05 \\
1.7136 E-05\end{array}$ & $\begin{array}{l}2.9692 E-03 \\
7.5302 E-03 \\
2.3433 E-03 \\
2.6852 E-03 \\
4.2532 E-05 \\
7.5734 E-04 \\
1.2908 E-04 \\
7.6091 E-05 \\
3.8046 E-05 \\
2.7992 E-05 \\
1.6674 E-05 \\
2.3210 E-02\end{array}$ \\
\hline & & & & Ue \\
\hline
\end{tabular}

3.8638E-02 3.8919E-02

$3.9210 E-02 \quad 3.9512 E-02 \quad 3.9826 E-02$

\begin{tabular}{|c|c|c|c|c|}
\hline $\begin{array}{r}5.57 \\
17.26 \\
5.18 \\
5.82 \\
0.05 \\
1.47 \\
0.40 \\
0.22 \\
0.11 \\
0.08 \\
0.05 \\
63.80\end{array}$ & $\begin{array}{r}5.99 \\
17.65 \\
5.36 \\
6.05 \\
0.06 \\
1.59 \\
0.38 \\
0.21 \\
0.11 \\
0.08 \\
0.05 \\
62.47\end{array}$ & $\begin{array}{r}6.45 \\
18.06 \\
5.54 \\
6.28 \\
0.08 \\
1.70 \\
0.36 \\
0.20 \\
0.10 \\
0.08 \\
0.04 \\
61.11\end{array}$ & $\begin{array}{r}6.93 \\
18.47 \\
5.71 \\
6.51 \\
0.09 \\
1.80 \\
0.34 \\
0.20 \\
0.10 \\
0.07 \\
0.04 \\
59.71\end{array}$ & $\begin{array}{r}7.46 \\
18.91 \\
5.88 \\
6.74 \\
0.11 \\
1.90 \\
0.32 \\
0.19 \\
0.10 \\
0.07 \\
0.04 \\
58.28\end{array}$ \\
\hline 100.00 & 100.00 & 100.00 & 100.00 & 100.00 \\
\hline
\end{tabular}




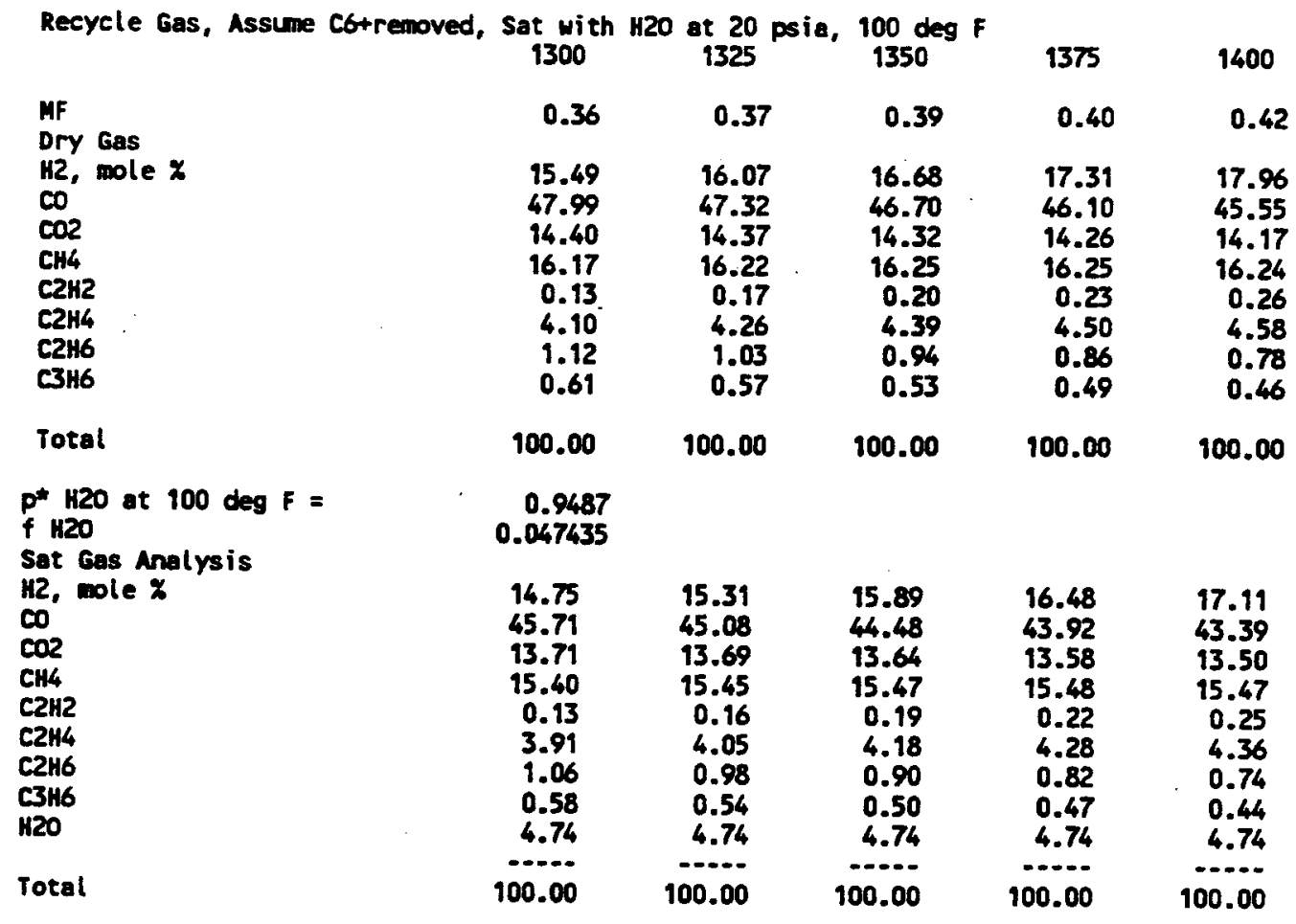




H2, lb/lb total feed
CO
CO2
CH4
C2H2
C2H4
C2H6
C3H6
C6H6
C1OH8
C14H10
H2O
Ash
Char, maF
TOtal
H2, moles/lb feed
CO
CO2
CH4
C2H2
C2H4
C2H6
C3H6
C6H6
C1OH8
C14H1O
H2O

H2, mole $x$
CO
CO2
CH4
C2H2
C2H6
C2H6
C3H6
C6H6
C10H8
C14H1O
H2O

\begin{tabular}{|c|c|c|c|c|}
\hline 1425 & 1450 & 1475 & 1500 & 1525 \\
\hline $\begin{array}{l}6.4850 E-03 \\
2.1770 E-01 \\
1.0690 E-01 \\
4.4890 E-02 \\
1.2728 E-03 \\
2.2468 E-02 \\
3.6645 E-03 \\
2.8896 E-03 \\
2.8896 E-03 \\
2.8896 E-03 \\
2.8896 E-03 \\
4.1093 E-01 \\
5.9191 E-03 \\
1.6821 E-01\end{array}$ & $\begin{array}{l}7.0252 E-03 \\
2.2483 E-01 \\
1.1065 E-01 \\
4.6714 E-02 \\
1.4415 E-03 \\
2.3629 E-02 \\
3.6421 E-03 \\
2.8072 E-03 \\
2.8072 E-03 \\
2.8072 E-03 \\
2.8072 E-03 \\
4.0344 E-01 \\
5.9191 E-03 \\
1.6168 E-01\end{array}$ & $\begin{array}{ll}7.6089 E-03 & 8 \\
2.3233 E-01 & 2 \\
1.1436 E-01 & 1 \\
4.8549 E-02 & 5 \\
1.6130 E-03 & 1 \\
2.4717 E-02 & 2 \\
3.2140 E-03 & 2 \\
2.7249 E-03 & 2 \\
2.7249 E-03 & 2 \\
2.7249 E-03 & 2 \\
2.7249 E-03 \\
3.9566 E-01 \\
5.9191 E-03 \\
1.5512 E-01\end{array}$ & $\begin{array}{l}8.2394 E-03 \\
2.4025 E-01 \\
1.1802 E-01 \\
5.0388 E-02 \\
1.7868 E-03 \\
2.5718 E-02 \\
2.9813 E-03 \\
2.6426 E-03 \\
2.6426 E-03 \\
2.6426 E-03 \\
2.6426 E-03 \\
3.8760 E-01 \\
5.9191 E-03 \\
1.4854 E-01\end{array}$ & $\begin{array}{l}8.9202 E-03 \\
2.4860 E-01 \\
1.2160 E-01 \\
5.2227 E-02 \\
1.9624 E-03 \\
2.6615 E-02 \\
2.7452 E-03 \\
2.5603 E-03 \\
2.5603 E-03 \\
2.5603 E-03 \\
2.5603 E-03 \\
3.7924 E-01 \\
5.9191 E-03 \\
1.4193 E-01\end{array}$ \\
\hline $.0000 E+00$ & $1.0000 E+00$ & $1.0000 E+00$ & $1.0000 E+00$ & $1.0000 E+00$ \\
\hline $\begin{array}{l}3.2171 E-03 \\
7.7722 E-03 \\
2.4291 E-03 \\
2.7981 E-03 \\
4.8883 E-05 \\
8.0088 E-04 \\
1.2189 E-04 \\
7.3983 E-05 \\
3.6992 E-05 \\
2.7217 E-05 \\
1.6212 E-05 \\
2.2810 E-02\end{array}$ & $\begin{array}{l}3.4851 E-03 \\
8.0266 E-03 \\
2.5143 E-03 \\
2.9119 E-03 \\
5.5360 E-05 \\
8.4228 E-04 \\
1.1449 E-04 \\
7.1875 E-05 \\
3.5938 E-05 \\
2.6461 E-05 \\
1.5750 E-05 \\
2.2394 E-02 \\
-\end{array}$ & $\begin{array}{l}3.7746 E-03 \\
8.2945 E-03 \\
2.5985 E-03 \\
3.0262 E-03 \\
6.1946 E-05 \\
8.8106 E-04 \\
1.0691 E-04 \\
6.9767 E-05 \\
3.4884 E-05 \\
2.5666 E-05 \\
1.5288 E-05 \\
2.1963 E-02 \\
\end{array}$ & $\begin{array}{l}4.0874 E-03 \\
8.5769 E-03 \\
2.6816 E-03 \\
3.1409 E-03 \\
6.8622 E-05 \\
9.1672 E-04 \\
9.9167 E-05 \\
6.7659 E-05 \\
3.3830 E-05 \\
2.4891 E-05 \\
1.4826 E-05 \\
2.1515 E-02 \\
-\end{array}$ & $\begin{array}{r}4.4251 E-03 \\
8.8751 E-03 \\
2.7629 E-03 \\
3.2555 E-03 \\
7.5365 E-05 \\
9.4871 E-04 \\
9.1312 E-05 \\
6.5551 E-05 \\
3.2776 E-05 \\
2.4115 E-05 \\
1.4364 E-05 \\
2.1051 E-02 \\
\end{array}$ \\
\hline DST- & 0494E-02 & 4.0852E-02 & $4.1227 E-02$ & $4.1622 E-02$ \\
\hline $\begin{array}{r}8.01 \\
19.36 \\
6.05 \\
6.97 \\
0.12 \\
1.99 \\
0.30 \\
0.18 \\
0.09 \\
0.07 \\
0.04 \\
56.81\end{array}$ & $\begin{array}{r}8.61 \\
19.82 \\
6.21 \\
7.19 \\
0.14 \\
2.08 \\
0.28 \\
0.18 \\
0.09 \\
0.07 \\
0.04 \\
55.30\end{array}$ & $\begin{array}{r}9.24 \\
20.30 \\
6.36 \\
7.41 \\
0.15 \\
2.16 \\
0.26 \\
0.17 \\
0.09 \\
0.06 \\
0.04 \\
53.76\end{array}$ & $\begin{array}{r}9.91 \\
20.80 \\
6.50 \\
7.62 \\
0.17 \\
2.22 \\
0.24 \\
0.16 \\
0.08 \\
0.06 \\
0.04 \\
52.19\end{array}$ & $\begin{array}{r}10.63 \\
21.32 \\
6.64 \\
7.82 \\
0.18 \\
2.28 \\
0.22 \\
0.16 \\
0.08 \\
0.06 \\
0.03 \\
50.58\end{array}$ \\
\hline & 100.00 & & & 100.00 \\
\hline
\end{tabular}




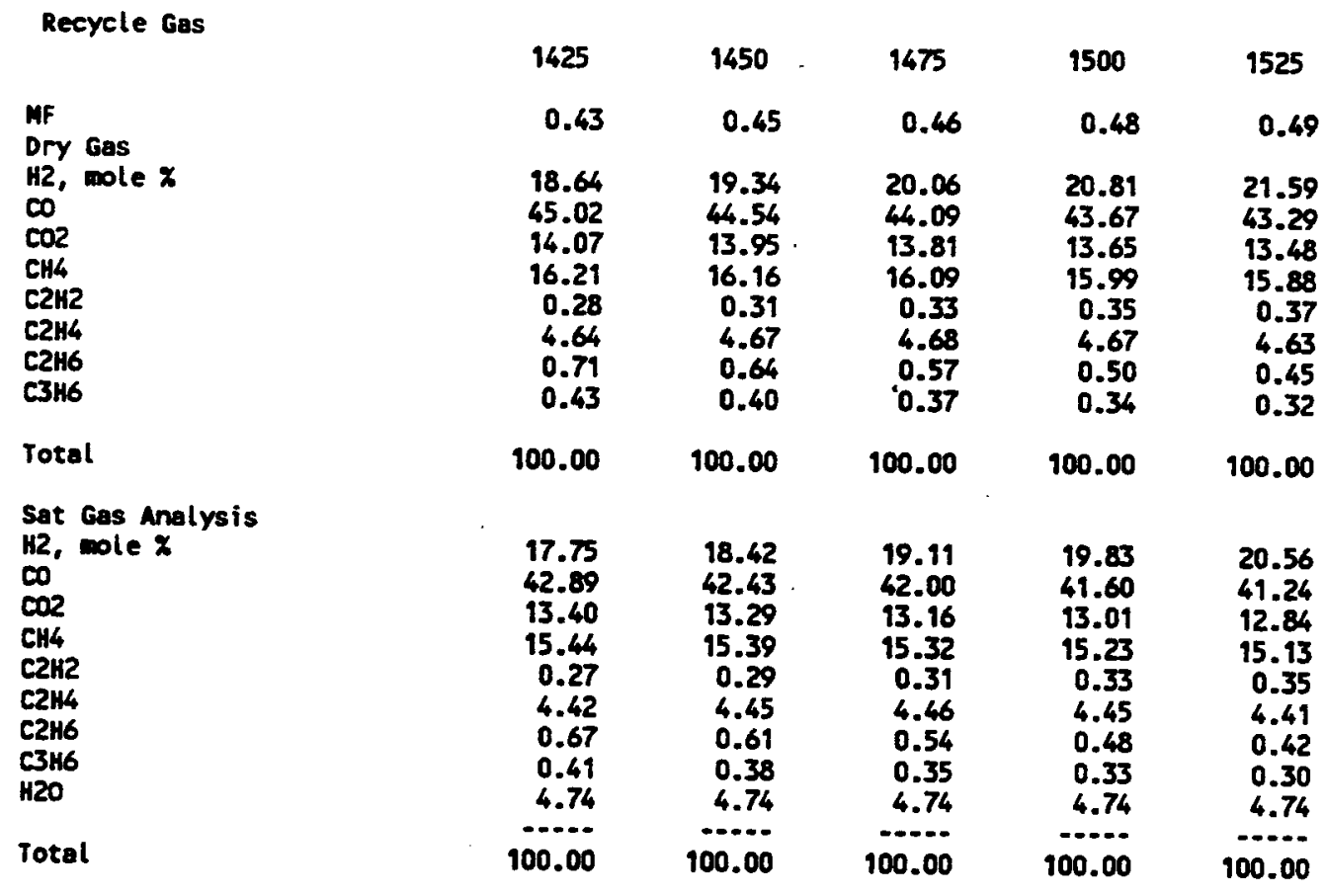




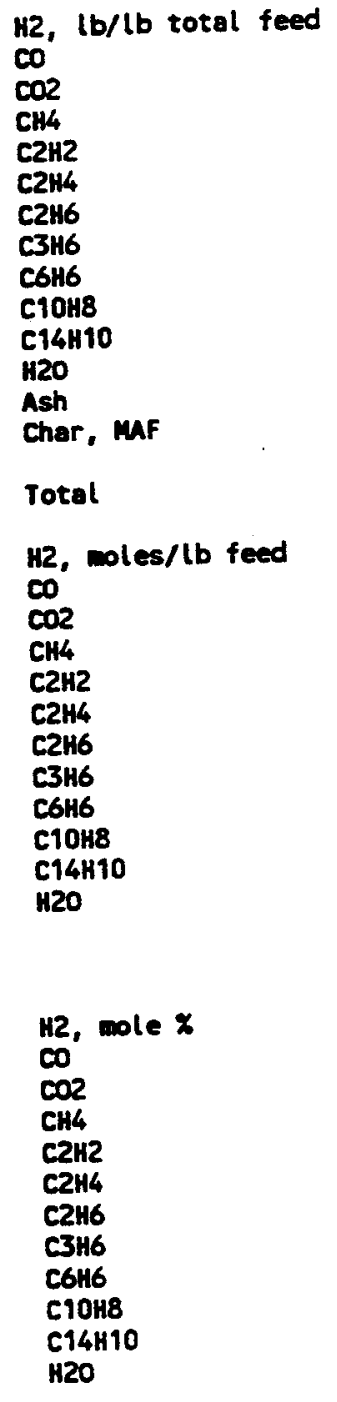

1550

9.6550E-03

2.5743E-01

1.2509E-01

5.4059E-02

2.1391E-03

2.7392E-02

2.5068E-03

2.4779E-03

2.4779E-03

$2.4779 E-03$

2.4779E-03

3.7060E-01

5.9191E-03

$1.3530 E-01$

$1.0000 E+00$

4.7897E-03

$9.1904 E-03$
$2.8422 E-03$

2.8422E-03

8.2152E-05

$9.7642 E-04$

8.3384E-05

6.3443E-05

3.1722E-05

2.3340E-05

1.3903E-05

2.0572E-02

4.2038E-02
$1.0448 E-02$

$1600 \quad 1625$

1.1303E-02 1.2226E-02 $1.3220 E-02$

2.6678E-01 2.7669E-01 2.8721E-01 2.9839E-01

$1.2846 E-01$ 1.3171E-01 1.3479E-01 1.3769E-01

$5.5879 E-02 \quad 5.7680 E-02 \quad 5.9455 E-02 \quad 6.1195 E-02$

$\begin{array}{llll}2.3163 E-03 & 2.4932 E-03 & 2.6690 E-03 & 2.8428 E-03\end{array}$

$\begin{array}{llll}2.3163 E-03 & 2.4912 E-02 & 2.8812 E-02 & 2.8909 E-02\end{array}$

$2.267 T E-03$ 2.0294E-03 1.7935E-03 1.5622E-03

$2.3956 E-03 \quad 2.3133 E-03 \quad 2.2309 E-03 \quad 2.1486 E-03$

2.3956E-03 2.3133E-03 2.2309E-03 $2.1486 E-03$

$2.3956 E-03 \quad 2.3133 E-03 \quad 2.2309 E-03 \quad 2.1486 E-03$

$2.3956 E-03 \quad 2.3133 E-03 \quad 2.2309 E-03 \quad 2.1486 E-03$

3.6168E-01 3.5266E-01 3.4296E-01 3.3317E-01

5.6191E-03 $5.9191 E-03$ 5.9191E-03 5.9191E-03

1.2864 E-01 $1.2196 E-01$ 1.1524E-01 1.0851E-01

$1.0000 E+00 \quad 9.0000 E+00 \quad 1.0000 E+00 \quad 1.0000 E+00$

$\begin{array}{llll}5.1830 E-03 & 5.6073 E-03 & 6.0649 E-03 & 6.5583 E-03\end{array}$

$\begin{array}{llll}.5241 E-03 & 9.8780 E-03 & 1.0254 E-02 & 1.0653 E-02\end{array}$

2.9190E-03 2.9926E-03 3.0627E-03 3.1286E-03

$\begin{array}{lll}3.0606 & 03 & 3.8145 E-03\end{array}$

8.8958E-05 9.5752E-05 $1.0250 E-04$ 1.0918E-04

0.0919E-04 $1.0163 E-03 \quad 1.0270 E-03 \quad 1.0305 E-03$

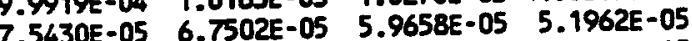

$6.1336 E-05$ 5.9228E-05 5.7120E-05 5.5012E-05

3.0668E-05 2.9614E-05 2.8560E-05 2.7506E-05

2.2564E-05 2.1789E-05 2.1013E-05 2.0238E-05

$1.3441 E-05$ 1.2979E-05 $1.2517 E-05 \quad 1.2055 E-05$

2.0076E-02 $1.9565 E-02$ 1.9037E-02 1.8494E-02

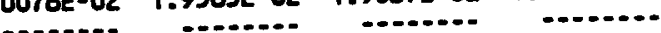

4.24TTE-02 4.2941E-02 4.3433E-02 4.3954E-02

\begin{tabular}{|c|c|c|c|c|}
\hline $\begin{array}{r}11.39 \\
21.86 \\
6.76 \\
8.02 \\
0.20 \\
2.32 \\
0.20 \\
0.15 \\
0.08 \\
0.06 \\
0.03 \\
48.94\end{array}$ & $\begin{array}{r}12.20 \\
22.42 \\
6.87 \\
8.20 \\
0.21 \\
2.35 \\
0.18 \\
0.14 \\
0.07 \\
0.05 \\
0.03 \\
47.26\end{array}$ & $\begin{array}{r}13.06 \\
23.00 \\
6.97 \\
8.37 \\
0.22 \\
2.37 \\
0.16 \\
0.14 \\
0.07 \\
0.05 \\
0.03 \\
45.56\end{array}$ & $\begin{array}{r}13.96 \\
23.61 \\
7.05 \\
8.53 \\
0.26 \\
2.36 \\
0.14 \\
0.13 \\
0.07 \\
0.05 \\
0.03 \\
43.83\end{array}$ & $\begin{array}{r}14.92 \\
24.24 \\
7.12 \\
8.68 \\
0.25 \\
2.34 \\
0.12 \\
0.13 \\
0.06 \\
0.05 \\
0.03 \\
42.07\end{array}$ \\
\hline 00 & 100.00 & 100.00 & 100.00 & 100.00 \\
\hline
\end{tabular}




Recycle gas
MF
Dry Gas
H2, mole $x$
CO
CO2
CH4
C2H2
C2H4
C2H6
C3H6
Total
Sat Gas Analysis
H2, mole $x$
CO
CO2
CH4
C2H2
C2H4
C2H6
C3H6
H2O
Total

\begin{tabular}{|c|c|c|c|c|}
\hline 1550 & $15 \pi$ & 1600 & 1625 & 1650 \\
\hline 0.51 & 0.53 & 0.54 & 0.56 & 0.58 \\
\hline $\begin{array}{r}22.38 \\
42.95 \\
13.28 \\
15.75 \\
0.38 \\
4.56 \\
0.39 \\
0.30\end{array}$ & $\begin{array}{r}23.21 \\
42.64 \\
13.07 \\
15.60 \\
0.40 \\
4.47 \\
0.34 \\
0.27\end{array}$ & $\begin{array}{r}24.05 \\
42.37 \\
12.84 \\
15.42 \\
0.41 \\
4.36 \\
0.29 \\
0.25\end{array}$ & $\begin{array}{r}24.92 \\
42.14 \\
12.59 \\
15.23 \\
0.42 \\
4.22 \\
0.25 \\
0.23\end{array}$ & $\begin{array}{r}25.82 \\
41.94 \\
12.32 \\
15.02 \\
0.43 \\
4.06 \\
0.20 \\
0.22\end{array}$ \\
\hline 100.00 & 100.00 & 100.00 & 100.00 & 100.00 \\
\hline $\begin{array}{r}21.32 \\
40.91 \\
12.65 \\
15.00 \\
0.37 \\
4.35 \\
0.37 \\
0.28 \\
4.74 \\
-\end{array}$ & $\begin{array}{r}22.11 \\
40.62 \\
12.45 \\
14.86 \\
0.38 \\
4.26 \\
0.32 \\
0.26 \\
4.74 \\
\ldots \ldots . .\end{array}$ & $\begin{array}{r}22.91 \\
40.36 \\
12.23 \\
14.69 \\
0.39 \\
4.15 \\
0.28 \\
0.24 \\
4.74 \\
\cdots\end{array}$ & $\begin{array}{r}23.74 \\
40.14 \\
11.99 \\
14.51 \\
0.40 \\
4.02 \\
0.23 \\
0.22 \\
4.74 \\
\cdots\end{array}$ & $\begin{array}{r}24.59 \\
39.95 \\
11.73 \\
14.30 \\
0.41 \\
3.86 \\
0.19 \\
0.21 \\
4.74 \\
\end{array}$ \\
\hline 100.00 & 100.00 & 100.00 & 100.00 & 100.00 \\
\hline
\end{tabular}




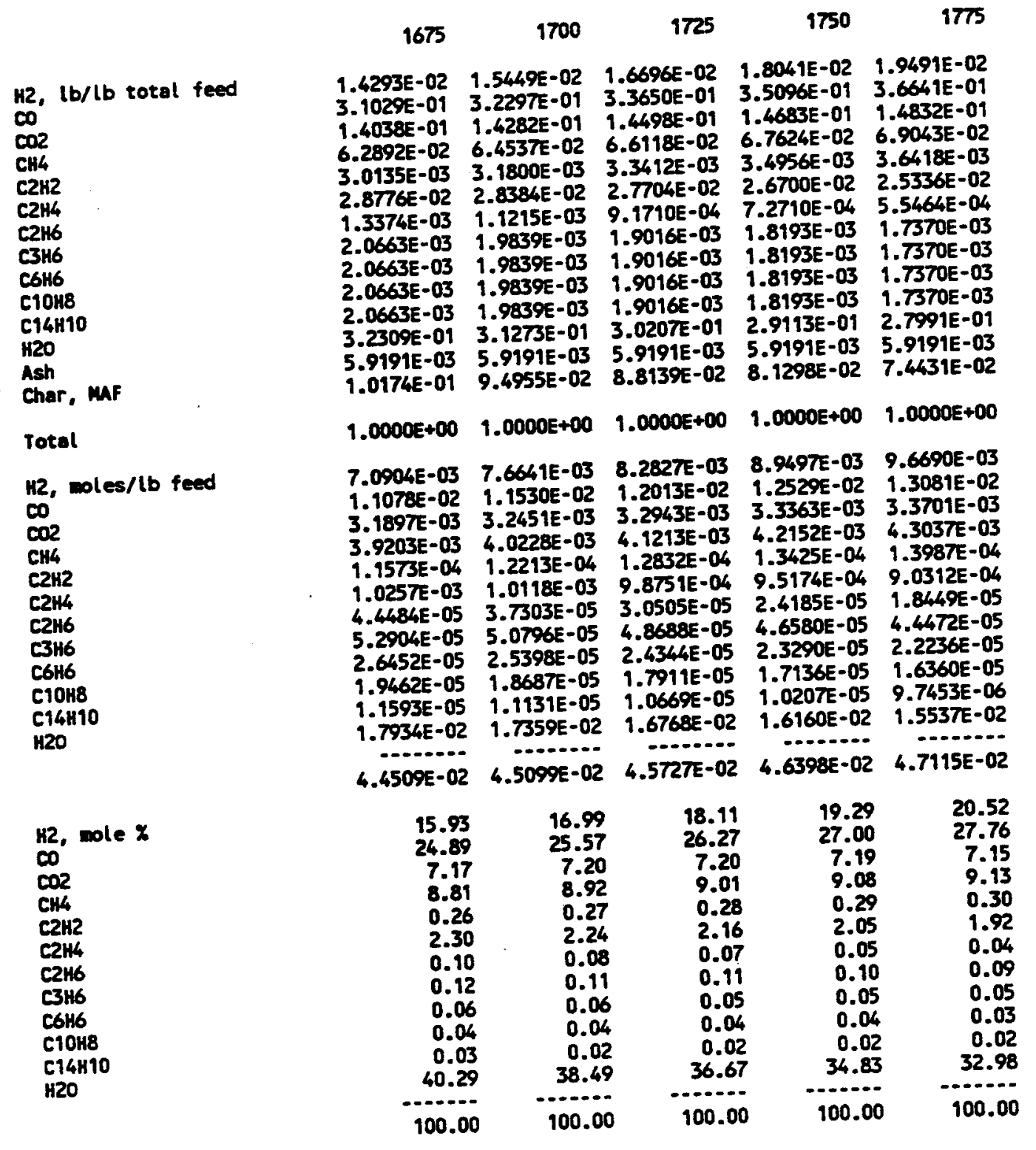




\begin{tabular}{|c|c|c|c|c|c|}
\hline \multicolumn{6}{|l|}{ Recycle Gas } \\
\hline & 1675 & 1700 & 1725 & 1750 & 1775 \\
\hline $\begin{array}{l}\text { MF } \\
\text { Dry Gas }\end{array}$ & 0.60 & 0.61 & 0.63 & 0.65 & 0.67 \\
\hline $\begin{array}{l}\text { H2, mole } \% \\
\text { CO } \\
\text { CO2 } \\
\text { CH4 } \\
\text { C2H2 } \\
\text { C2H4 } \\
\text { C2H6 } \\
\text { C3H6 }\end{array}$ & $\begin{array}{l}26.74 \\
41.78 \\
12.03 \\
14.78 \\
0.44 \\
3.87 \\
0.17 \\
0.20\end{array}$ & $\begin{array}{l}27.68 \\
41.65 \\
11.72 \\
14.53 \\
0.44 \\
3.65 \\
0.13 \\
0.18\end{array}$ & $\begin{array}{l}28.65 \\
41.56 \\
11.40 \\
14.26 \\
0.44 \\
3.42 \\
0.11 \\
0.17\end{array}$ & $\begin{array}{l}29.65 \\
41.51 \\
11.05 \\
13.96 \\
0.44 \\
3.15 \\
0.08 \\
0.15\end{array}$ & $\begin{array}{l}30.67 \\
41.49 \\
10.69 \\
13.65 \\
0.44 \\
2.86 \\
0.06 \\
0.14\end{array}$ \\
\hline Total & 100.00 & 100.00 & 100.00 & 100.00 & 100.00 \\
\hline $\begin{array}{l}\text { Sat Gas Analysis } \\
\text { H2, mole } X \\
\text { CO } \\
\text { CO2 } \\
\text { CH4 } \\
\text { C2 H2 } \\
\text { C2 H4 } \\
\text { C2H6 } \\
\text { C3H6 } \\
\text { H2O }\end{array}$ & $\begin{array}{l}25.47 \\
39.79 \\
11.46 \\
14.08 \\
0.42 \\
3.68 \\
0.16 \\
0.19 \\
4.74\end{array}$ & $\begin{array}{l}26.37 \\
39.67 \\
11.17 \\
13.84 \\
0.42 \\
3.48 \\
0.13 \\
0.17 \\
4.74\end{array}$ & $\begin{array}{l}27.29 \\
39.59 \\
10.86 \\
13.58 \\
0.42 \\
3.25 \\
0.10 \\
0.16 \\
4.74\end{array}$ & $\begin{array}{l}28.24 \\
39.54 \\
10.53 \\
13.30 \\
0.42 \\
3.00 \\
0.08 \\
0.15 \\
4.74\end{array}$ & $\begin{array}{r}29.21 \\
39.52 \\
10.18 \\
13.00 \\
0.42 \\
2.73 \\
0.06 \\
0.13 \\
4.74\end{array}$ \\
\hline Total & 100.00 & 100.00 & 100.00 & 100.00 & 100.00 \\
\hline
\end{tabular}




\begin{tabular}{|c|c|c|c|}
\hline & 1800 & 1825 & 1850 \\
\hline $\begin{array}{l}\text { H2, lb/lb total feed } \\
\text { CO } \\
\text { CO2 } \\
\text { CH4 } \\
\text { C2H2 } \\
\text { C2H4 } \\
\text { C2H6 } \\
\text { C3H6 } \\
\text { C6H6 } \\
\text { C1OH8 } \\
\text { C14H10 } \\
\text { H2O } \\
\text { Ash } \\
\text { Char, MAF }\end{array}$ & $\begin{array}{l}2.1055 E-02 \\
3.8296 E-01 \\
1.4941 E-01 \\
7.0362 E-02 \\
3.7782 E-03 \\
2.3571 E-02 \\
4.0320 E-04 \\
1.6546 E-03 \\
1.656 E-03 \\
1.6546 E-03 \\
1.6546 E-03 \\
2.6839 E-01 \\
5.9191-03 \\
6.7538 E-02\end{array}$ & $\begin{array}{l}2.2742 E-02 \\
4.0069 E-01 \\
1.505 E-01 \\
7.1964 E-02 \\
3.9029 E-03 \\
2.1361 E-02 \\
2.7665 E-04 \\
1.5723 E-03 \\
1.5723 E-03 \\
1.5723 E-03 \\
1.5723 E-03 \\
2.5659 E-01 \\
5.9191 E-03 \\
6.0619 E-02\end{array}$ & $\begin{array}{l}2.4562 E-02 \\
4.1973 E-01 \\
1.5018 E-01 \\
7.2633 E-02 \\
4.0139 E-03 \\
1.8656 E-02 \\
1.7925 E-04 \\
1.4900 E-03 \\
1.4900 E-03 \\
1.4900 E-03 \\
1.4900 E-03 \\
2.4450 E-01 \\
5.9191 E-03 \\
5.3674 E-02\end{array}$ \\
\hline Total & $1.0000 E+00$ & $9.0000 E+00$ & $1.0000 E+00$ \\
\hline $\begin{array}{l}\text { H2, moles/lb feed } \\
\text { CO } \\
\text { CO2 } \\
\text { CH4 } \\
\text { C2H2 } \\
\text { C2H4 } \\
\text { C2H6 } \\
\text { C3H6 } \\
\text { C6H6 } \\
\text { C1OHB } \\
\text { C114H1O } \\
\text { H2O }\end{array}$ & $\begin{array}{l}1.0445 E-02 \\
1.3672 E-02 \\
3.3949 E-03 \\
4.3859 E-03 \\
1.4510 E-04 \\
8.4022 E-04 \\
1.3411 E-05 \\
4.2364 E-05 \\
2.1182 E-05 \\
1.5585 E-05 \\
9.2834 E-06 \\
1.4898 E-02 \\
4 . \cdots \cdots \cdots-0 \\
4.7883 E-02\end{array}$ & $\begin{array}{l}1.1282 E-02 \\
1.4305 E-02 \\
3.4094 E-03 \\
4.4608 E-03 \\
1.4989 E-04 \\
7.6143 E-04 \\
9.2020 E-06 \\
4.0256 E-05 \\
2.0128 E-05 \\
1.4809 E-05 \\
8.8215 E-06 \\
1.4243 E-02 \\
. \ldots 13-02 \\
4.8704 E-02\end{array}$ & $\begin{array}{l}1.2185 E-02 \\
1.4985 E-02 \\
3.4126 E-03 \\
4.5274 E-03 \\
1.5415 E-04 \\
6.6500 E-04 \\
5.9625 E-06 \\
3.8148 E-05 \\
1.9074 E-05 \\
1.4034 E-05 \\
8.3596 E-06 \\
1.3572 E-02 \\
1 \ldots \ldots-0 . \\
4.9586 E-02\end{array}$ \\
\hline 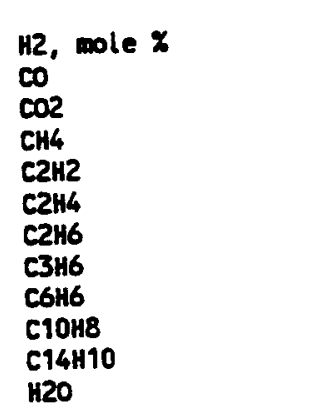 & $\begin{array}{r}21.81 \\
28.55 \\
7.09 \\
9.16 \\
0.30 \\
1.75 \\
0.03 \\
0.09 \\
0.04 \\
0.03 \\
0.02 \\
31.11\end{array}$ & $\begin{array}{r}23.16 \\
29.37 \\
7.00 \\
9.16 \\
0.31 \\
1.56 \\
0.02 \\
0.08 \\
0.04 \\
0.03 \\
0.02 \\
29.24\end{array}$ & $\begin{array}{r}24.57 \\
30.22 \\
6.88 \\
9.13 \\
0.31 \\
1.34 \\
0.01 \\
0.08 \\
0.04 \\
0.03 \\
0.02 \\
27.37\end{array}$ \\
\hline & 100.00 & 100.00 & 100.00 \\
\hline
\end{tabular}




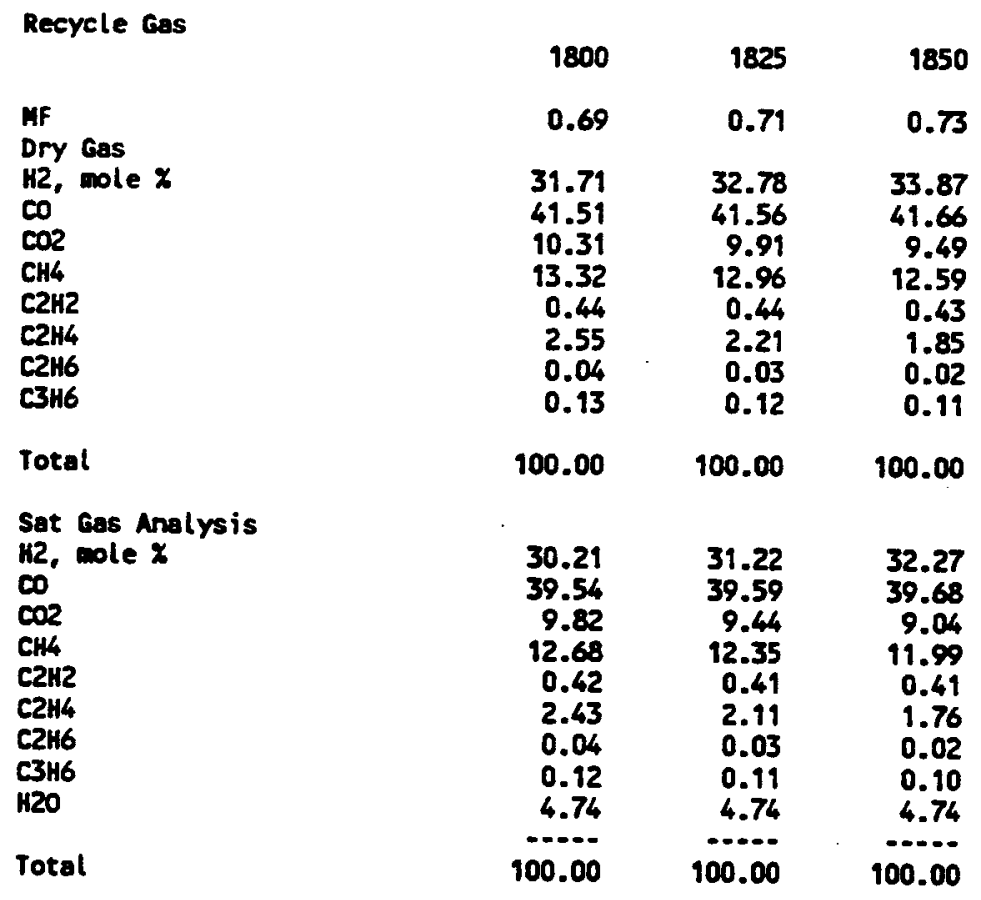


APPENDIX 11

116 


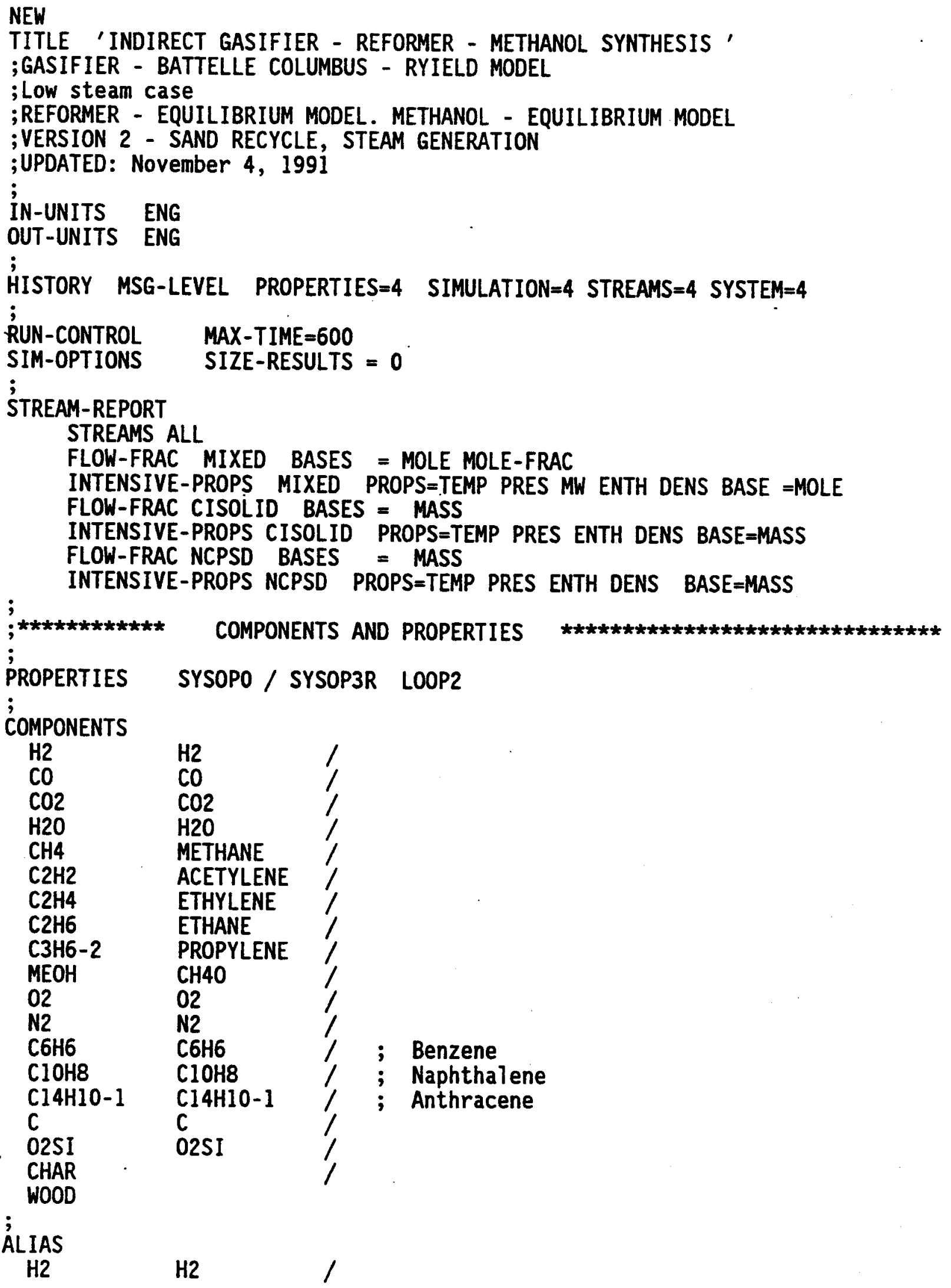




\begin{tabular}{|c|c|}
\hline $\begin{array}{l}\mathrm{CO} \\
\mathrm{CO} 2 \\
\mathrm{H} 2 \mathrm{O} \\
\mathrm{CH} 4 \\
\mathrm{C} 2 \mathrm{H} 2 \\
\mathrm{C} 2 \mathrm{H} 4 \\
\mathrm{C} 2 \mathrm{H6} \\
\mathrm{C} 3 \mathrm{H} 6-2 \\
\mathrm{MEOH} \\
\text { O2 } \\
\mathrm{N} 2 \\
\mathrm{C} 6 \mathrm{H} 6 \\
\mathrm{C} 1 \mathrm{OH} 8 \\
\mathrm{C} 14 \mathrm{H} 10-1 \\
\mathrm{C} \\
\text { O2SI } \\
\text { CHAR }\end{array}$ & $\begin{array}{l}\mathrm{CO} \\
\mathrm{CO} 2 \\
\mathrm{H} 2 \mathrm{O} \\
\mathrm{CH} 4 \\
\mathrm{C} 2 \mathrm{H} 2 \\
\mathrm{C} 2 \mathrm{H} 4 \\
\mathrm{C} 2 \mathrm{H} 6 \\
\mathrm{C} 3 \mathrm{H} 6-2 \\
\mathrm{CH} 40 \\
02 \\
\mathrm{~N} 2 \\
\mathrm{C} 6 \mathrm{H} 6 \\
\mathrm{C} 10 \mathrm{H} 8 \\
\mathrm{C} 14 \mathrm{H} 10-1 \\
\mathrm{C} \\
02 \mathrm{SI}\end{array}$ \\
\hline
\end{tabular}

; Attr-Comps CHAR Proxanal Ultanal Sulfanal Coalmisc Genanal

Attr-Comps WOOD Proxanal Ultanal Sulfanal Coalmisc Genanal

;

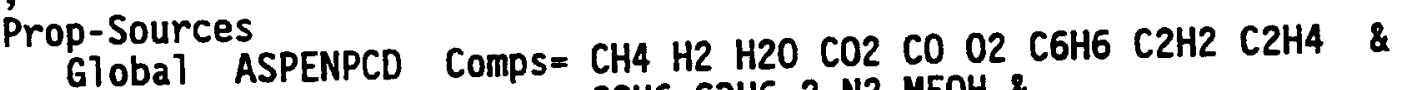

C2H6 C3H6-2 N2 MEOH \&

Global DIPPR Comps $=$ C10H8 C14H10-1 C O2SI

íc-props wood enthalpy hcjlboie / density dnstygen

Nc-props char enthalpy hcjlboie / density dnstygen

;

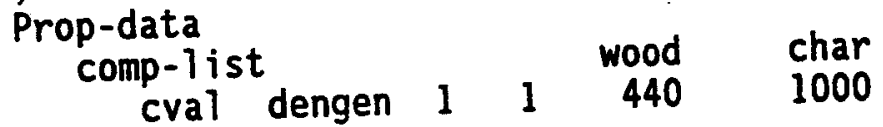

;

Prop-data
Prop-List BOIEC

$\begin{array}{ccccccc} & & & & & & \\ \text {; } & H & \text { S } & 0 & \text { N } & \text { BIAS } \\ \text { Pval Wood } & 154.8761 & 122.50632 & 0.0 & 0.0 & 0.0 & 0.0\end{array}$

$\begin{array}{lllllll}\text { Pval Char } 154.8761 & 122.50632 & 0.0 & 0.0 & 0.0 & 0.0\end{array}$

Prop-Data

PVAL C $12.01115 / 2.2 / 0 . / 0 . / 0.00530 .00 .00 .00 .00 .03000$. / \& $1.7154 \mathrm{e}+4 \quad 4.268 \quad 0.0 \quad 0.0-2.786 \mathrm{e}+8 \quad 0.03000 .0$

Pval 02SI $60.086 / 2.2 / 0 . / 0 . / .02726$ 0.0.0.0.0. $3000 . / \&$ 37713 0. 0. 0. 0. 0. 3000 .

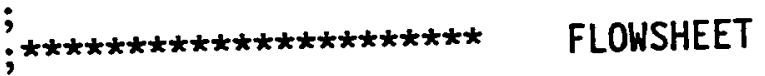

;

FLOWSHEET DRY

DRYER

STORE

IN $=$ WWOOD DRYGAS QNET
IN $=$ DWOOD

OUT = WETGAS DWOOD

QDRYER

OUT $=$ DWOOD2

QSTORE 


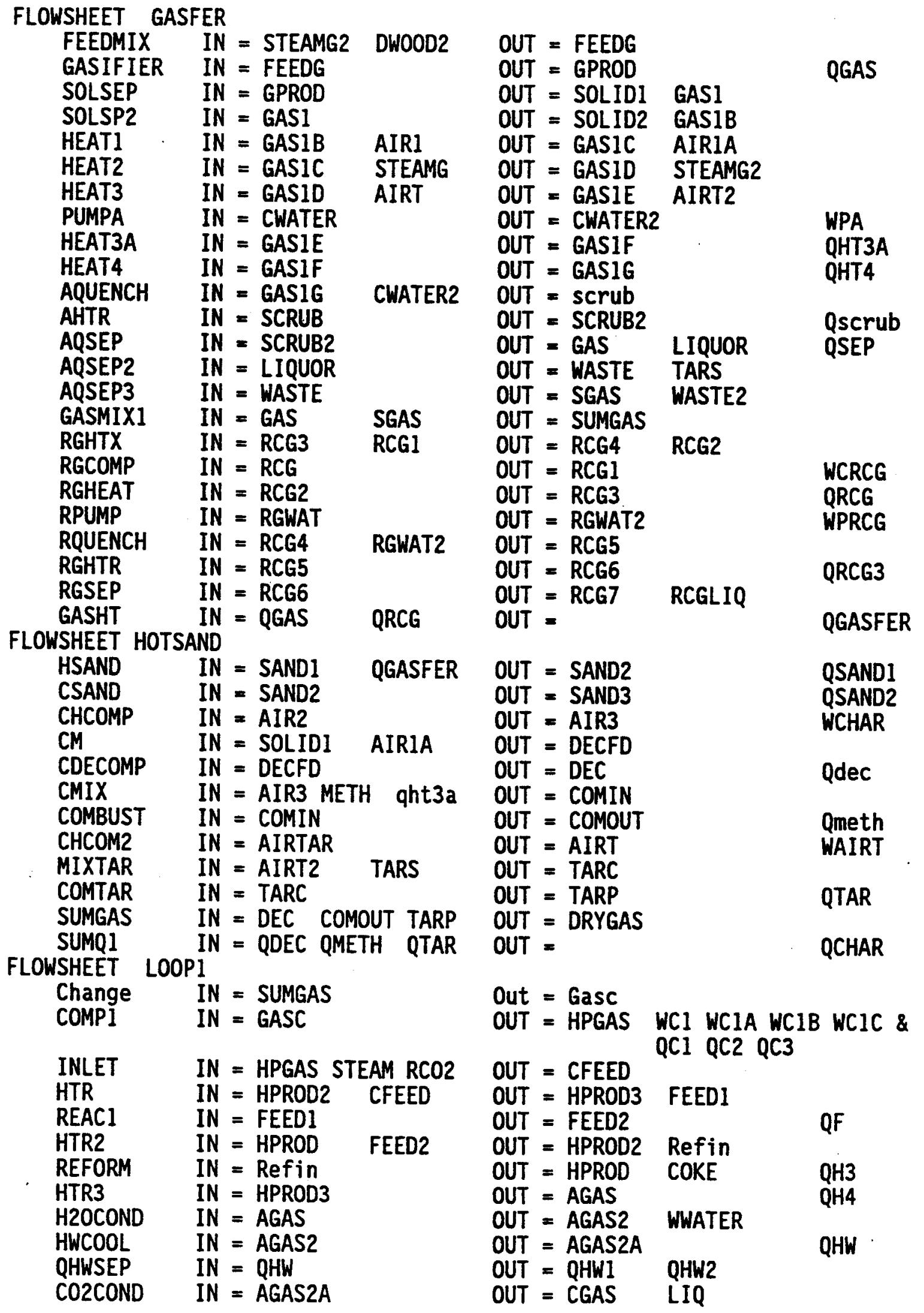




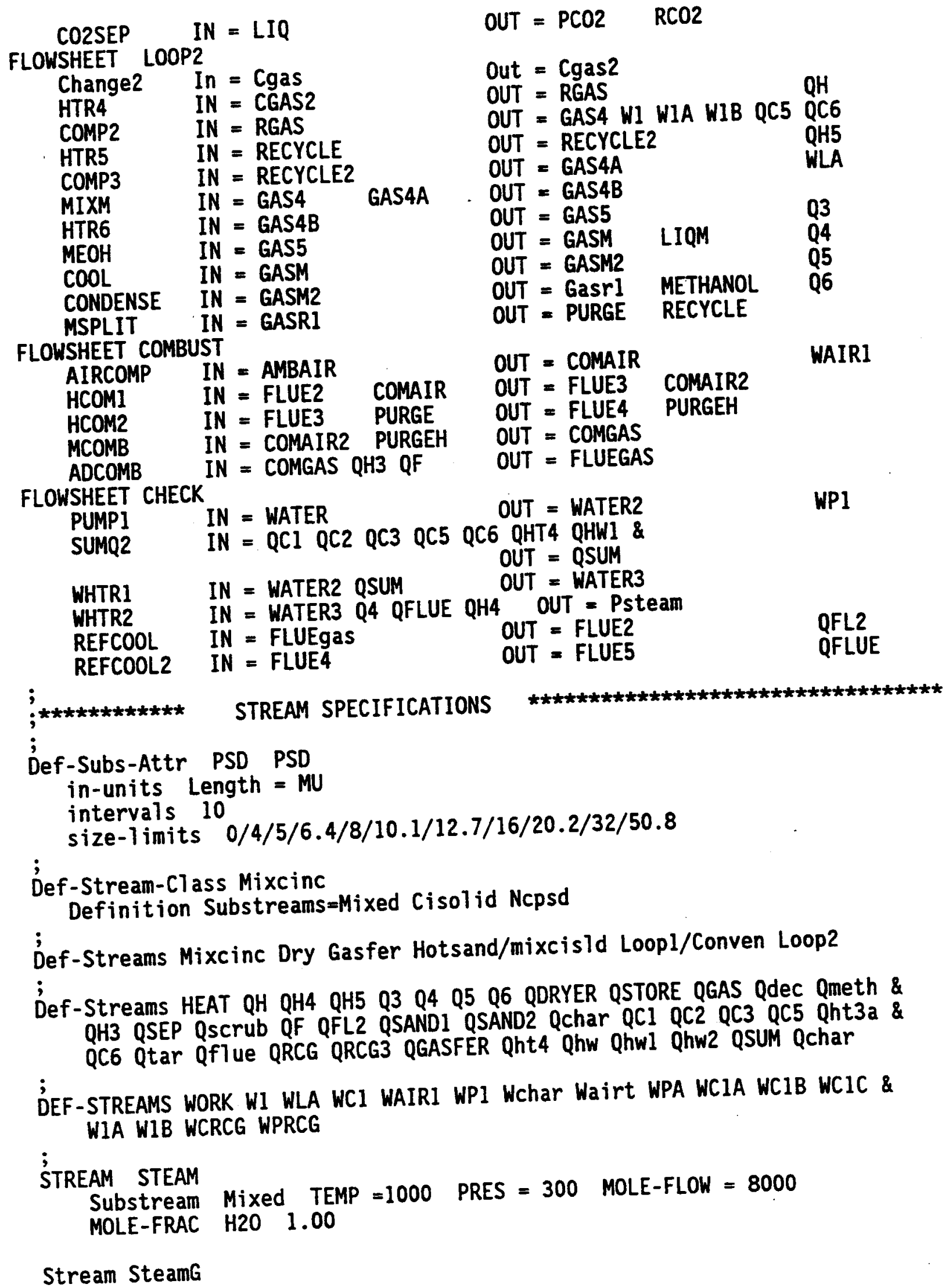


Substream Mixed Temp $=1000$ Pres $=25$ Mass $-f 70 \mathrm{w}=66360.340$

Mass-frac H2O 1.00

;

Stream Cwater

Substream Mixed Temp $=50 \quad P=14.7$ Mole-flow $=10000$

Mole-frac H2O 1.00

;

Stream water

Substream Mixed Temp $=50 \quad P=25$ Mole-flow $=11750$

Mole-frac $\mathrm{H} 2 \mathrm{O} \quad 1.00$

;

Stream Ambair

Substream Mixed Temp $=80$ Pres $=14.7$ Mole-flow $=5500$

Mole-frac $020.21 /$ N2 0.79

;

Stream Airl

Substream Mixed Temp $=80$ Pres $=25$ Mass - flow $=100$

Mass-frac 020.2329 / N2 0.7671

;

Stream Air2

Substream Mixed Temp $=80$ Pres $=14.7$ Mole-flow $=200$

Mole-frac $020.21 /$ N2 0.79

;

Stream Meth

Substream Mixed Temp $=80$ Pres $=14.7$ Mole-flow $=25$

Mole-frac MEOH 1.0 ;

Stream Airtar

Substream Mixed Temp $=80$ Pres $=14.7$ Mole-flow $=1250$

Mole-frac $020.21 /$ N2 0.79

;

Stream RCG

Substream Mixed Temp $=100$ Pres $=20$ Mole-flow $=4900.0$

Mole-frac H2 0.2547 / CO 0.3979 / CO2 0.1146 / CH4 0.1408 / \&

$\mathrm{C} 2 \mathrm{H} 20.0042$ / $\mathrm{C} 2 \mathrm{H} 40.0368$ / $\mathrm{C} 2 \mathrm{H} 60.0016 / \&$

C3H6-2 $0.0019 / \mathrm{H} 200.0474$

;

Stream Rgwat

Substream Mixed Temp $=50 \mathrm{P}=14.696$ Mole-flow $=1000$

Mole-frac H2O 1.0 ;

Stream Wwood Mixed Temp $=80$ Pres $=14.7$ Mass $-f 70 w=149693.674$

Mass-flow H2O 149693.674

Substream NCPSD Temp $=80$ Pres $=14.7$ Mass $-f 70 w=183639.660$

Mass-flow Wood 183639.660 / Char le-10

Subs-attr PSD Frac $=0.593 \quad 0.001 \quad 0.011 \quad 0.026 \quad 0.033 \quad 0.04 \quad 0.052 \&$

$\begin{array}{llll}0.072 & 0.140 & 0.032\end{array}$

Comp-Attr Wood Proxanal (9.24 15.3783 .440 .89$) /$

U7tanal ( $0.8950 .896 .04 \quad 0.170 .03 \quad 0.0941 .90) /$

Sulfanal $(0.03 \quad 0.03 \quad 0.03) /$

Coalmisc (8539. 0.00 .00 .00 .0$) /$

Genanal (100.0 $0.0 \quad 0.0 \quad 0.0 \quad 0.0 \quad 0.0 \quad 0.0 \quad 0.0 \quad 0.0 \quad 0.0)$ 


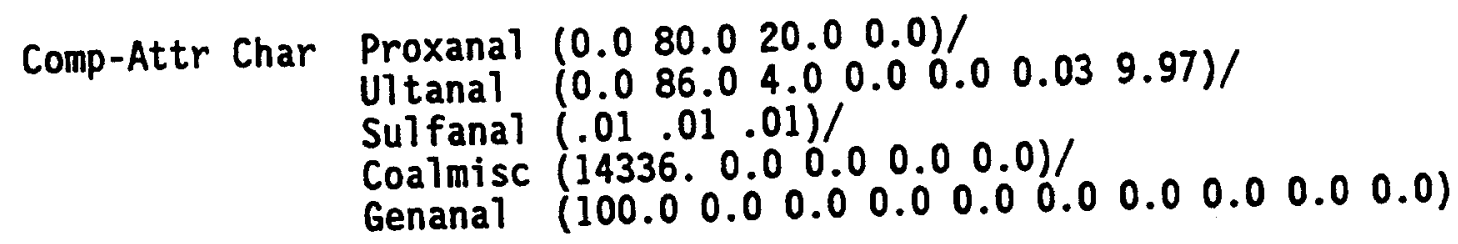

;

Stream Sandl

Substream Mixed Temp $=1975 \quad P=25$ Mole-flow=0.01

Mole-frac N2 1.00

Substream Cisolid Temp $=1975$ P $=25$ Mass $-f 10 w=6 E+6$

Mass-frac 02SI 1.00

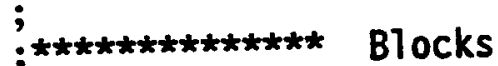

;

B' lock Dryer Flash2

Param Temp $=220$ Pres $=14.7$ maxit $=100$

;

Block Store Heater

Param Temp $=220$

;

BTock Feedmix Mixer

Param Pres $=20$

;

Block Gasifier Ryield

Properties Option=SYSOPO

Param Temp $=1675$ Pres $=20$

Massyield Ssid $=$ Mixed

Ssid $=$ Mixed

Comp $=\mathrm{H} 2$

$\mathrm{Va}=1.4293 \mathrm{E}-02 /$

Ssid $=$ Mixed

Comp $=\mathrm{CO}$

$\mathrm{Va}=3.1029 \mathrm{E}-01 /$

Ssid $=$ Mixed

Comp $=\mathrm{CO} 2$

$\mathrm{Va}]=1.4038 \mathrm{E}-01 /$

Ssid $=$ Mixed

Comp $=\mathrm{CH} 4$

$\mathrm{Val}=6.2892 \mathrm{E}-02 /$

Ssid $=$ Mixed

Comp $=\mathrm{C} 2 \mathrm{H} 2$

$\mathrm{Va}=3.0135 \mathrm{E}-03 /$

Ssid $=$ Mixed

Ssid $=$ Mixed

Comp $=\mathrm{C} 2 \mathrm{H} 4$

$\mathrm{Val}=2.8776 \mathrm{E}-02 /$

Comp $=\mathrm{C} 2 \mathrm{H} 6$

Ssid $=$ Mixed

Comp $=$ C3H6-2

$\mathrm{Va}=1.3374 \mathrm{e}-03 /$

Ssid $=$ Mixed

Ssid $=$ Mixed

Ssid = Mixed

Comp $=\mathrm{C} 6 \mathrm{H} 6$

$\mathrm{Va}]=2.0663 \mathrm{E}-03 /$

Comp $=\mathrm{C} 10 \mathrm{H} 8$

$\mathrm{Va}]=2.0663 \mathrm{E}-03 /$

Comp $=$ C14H10-1

$\mathrm{Va}]=2.0663 \mathrm{E}-03 /$

Ssid $=$ Cisolid

Comp $=\mathrm{H} 2 \mathrm{O}$

$\mathrm{Va}=2.0663 \mathrm{E}-03 /$

Ssid = Ncpsd

Comp $=02 S I$

Ssid $=$ Ncpsd

Comp $=$ Char

$\mathrm{Val}=3.2309 \mathrm{E}-01 /$

$\mathrm{Va}=5.9191 \mathrm{E}-03 /$

$\mathrm{Va}]=1.0174 \mathrm{E}-01 /$

$\mathrm{Va}]=0.0$

;

Block Solsep Sep2

Frac Subs=Ncpsd Subs=Mixed

Comp $=$ Wood

Flash-specs Gasl Pres $=20$

;

B'lock Solsp2 Sep2

Frac Subs $=$ Cisolid Stream $=$ Solid2 Comp $=02 S 1 \quad$ Frac $=1.0$ I

Flash-specs Gaslb Pres $=20$

; 


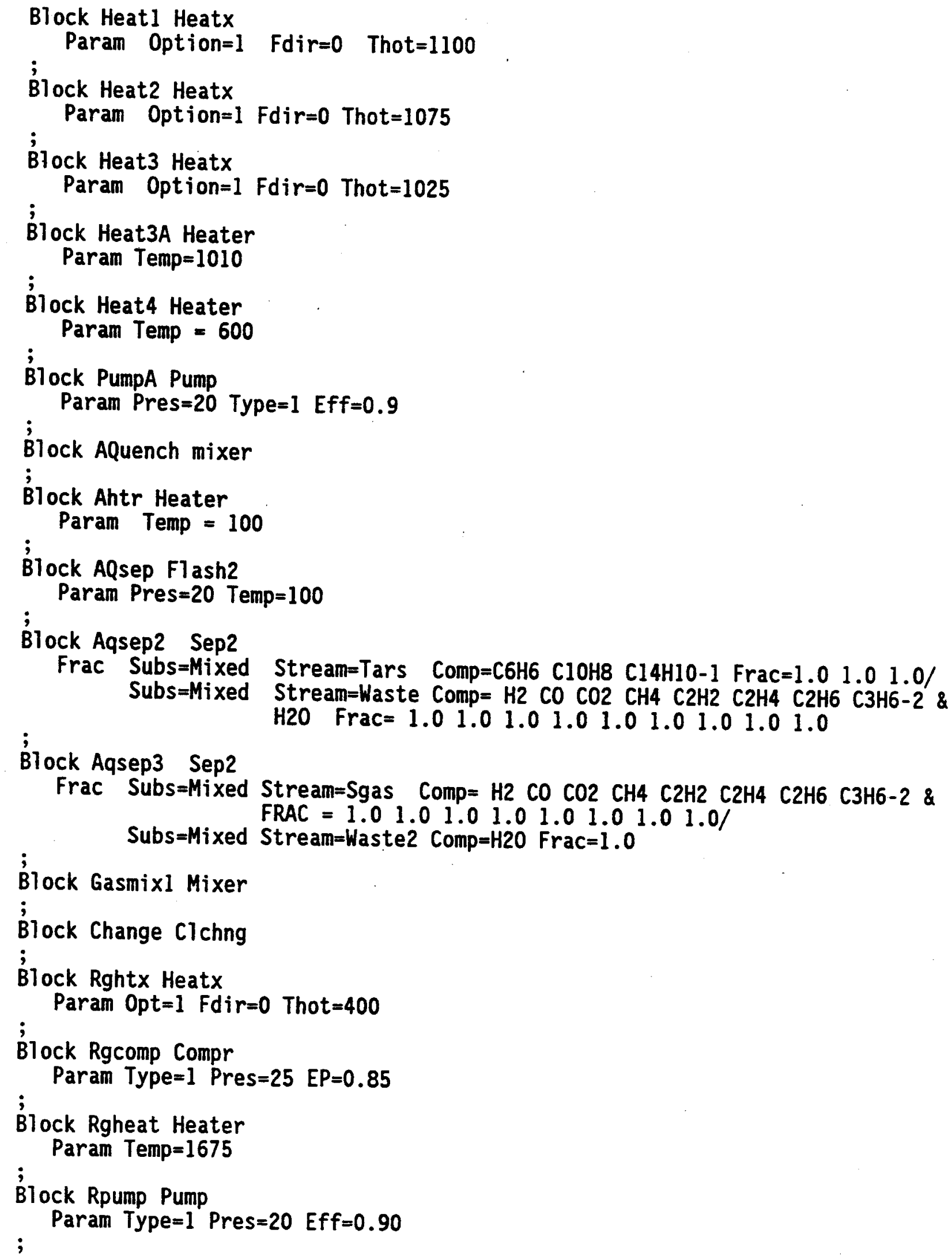

Frac Subs=Mixed Stream $=$ Tars Comp $=$ C6H6 $\mathrm{C} 10 \mathrm{H} 8 \mathrm{C} 14 \mathrm{H} 10-1$ Frac $=1.01 .01 .0 /$ Subs=Mixed Stream=Waste Comp $=\mathrm{H} 2 \mathrm{CO} \mathrm{CO}_{2} \mathrm{CH}_{4} \mathrm{C}_{2} \mathrm{H} 2 \mathrm{C}_{2} \mathrm{H} 4 \mathrm{C}_{2} \mathrm{H} 6 \mathrm{C} 3 \mathrm{H} 6-2$ \& H2O $\mathrm{Frac}=1.01 .01 .01 .01 .01 .01 .01 .01 .0$ ;

Block Aqsep3 Sep2

Frac Subs =Mixed Stream=Sgas Comp $=\mathrm{H} 2 \mathrm{CO} \mathrm{CO}_{2} \mathrm{CH}_{4} \mathrm{C} 2 \mathrm{H} 2 \mathrm{C}_{2} \mathrm{H} 4 \mathrm{C} 2 \mathrm{H} 6 \mathrm{C}_{3} \mathrm{H} 6-2$ \& $F R A C=1.01 .01 .01 .01 .01 .01 .01 .0 \%$

Subs $=$ Mixed Stream=Waste2 Comp $=$ H2O Frac $=1.0$

;

Bilock Gasmixl Mixer

;

Block Change Clchng

;

BTock Rghtx Heatx

Param Opt $=1$ Fdir $=0$ Thot $=400$

;

Block Rgcomp Compr

Param Type $=1$ Pres $=25$ EP $=0.85$

;

Block Rgheat Heater

Param Temp $=1675$

;

B́lock Rpump Pump

Param Type $=1$ Pres $=20$ Eff $=0.90$ ; 


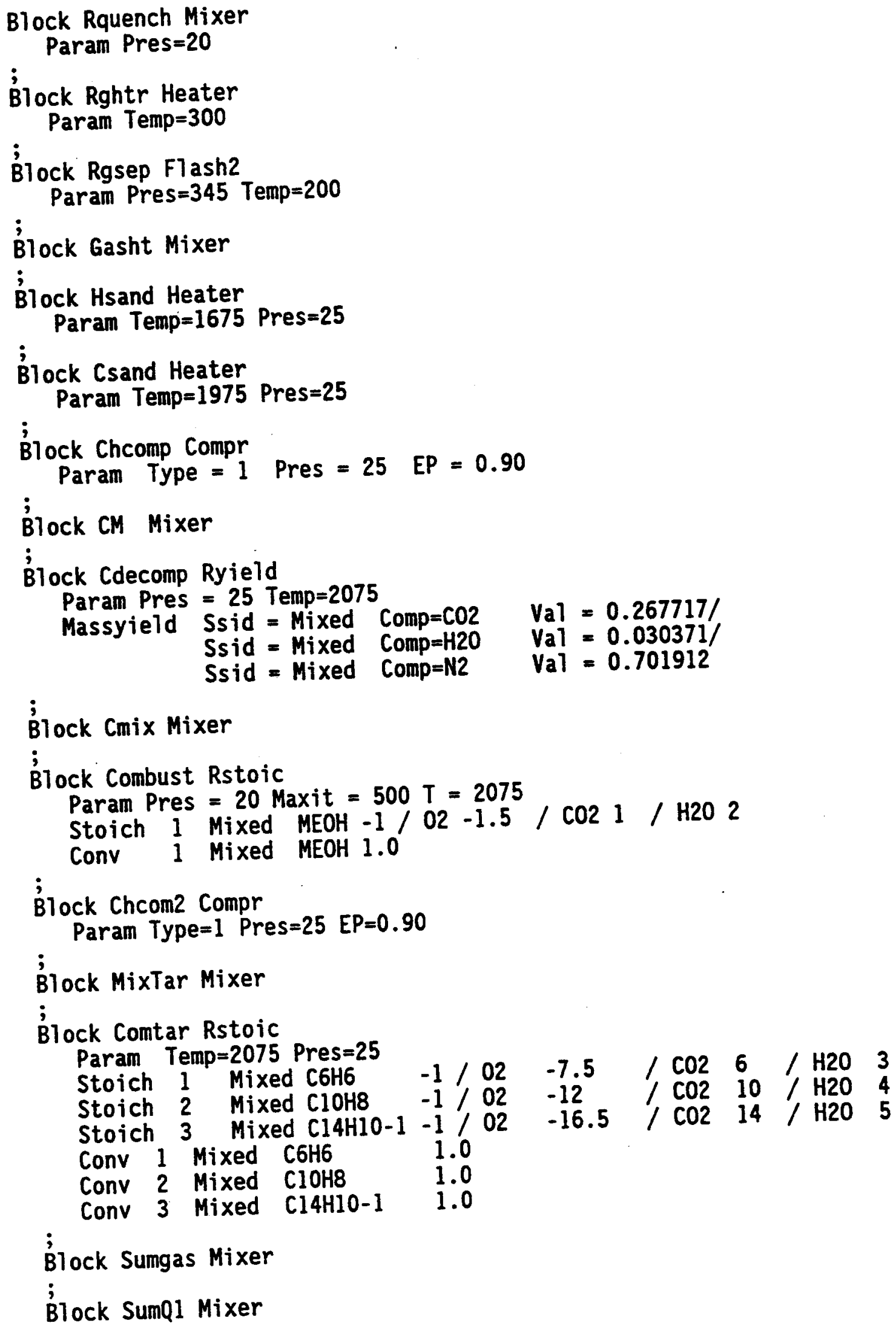




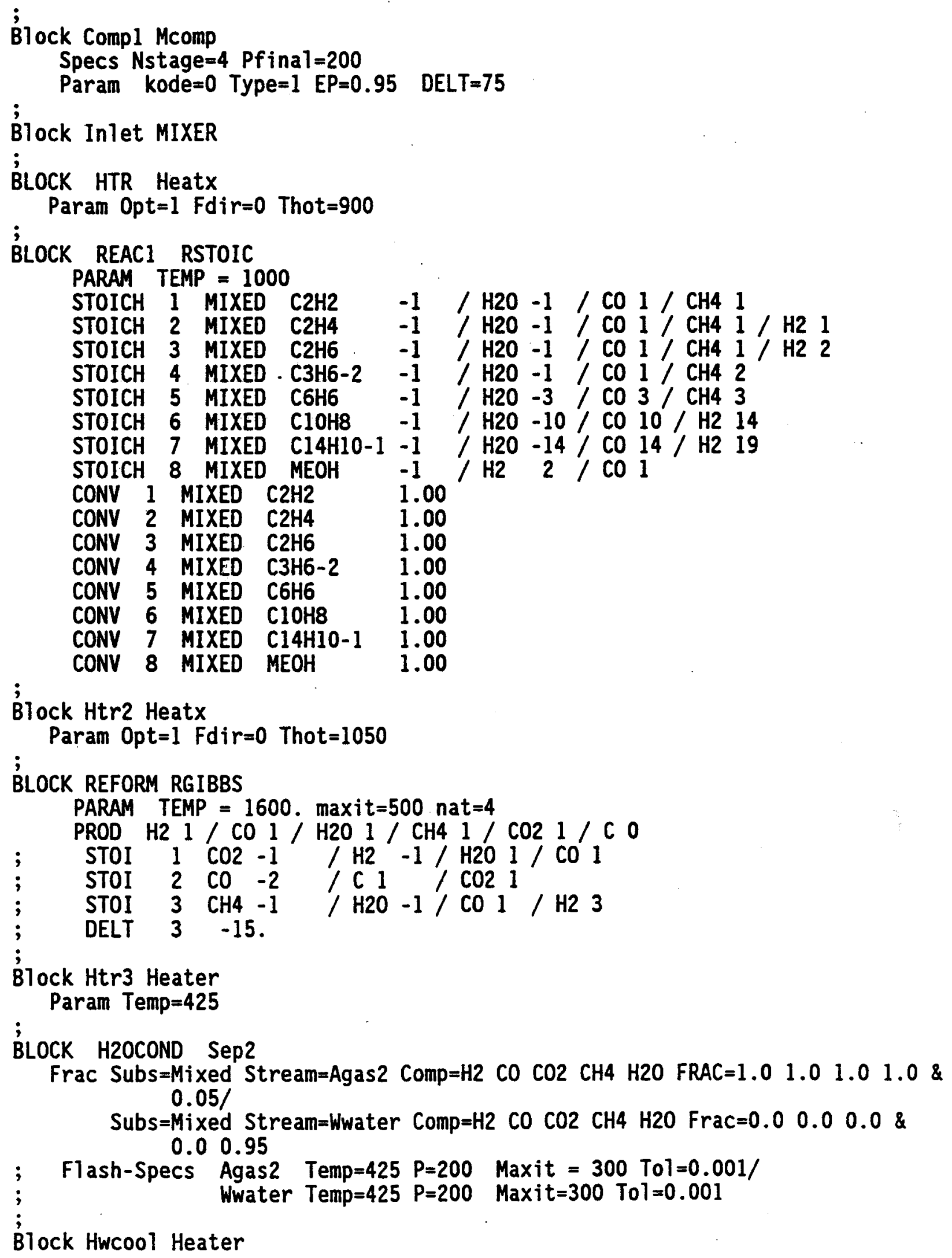

Frac Subs=Mixed Stream=Agas2 Comp=H2 CO CO2 CH4 H2O FRAC=1.0 $1.01 .01 .0 \&$ $0.05 /$

Subs=Mixed Stream=Wwater $\mathrm{Comp}=\mathrm{H} 2 \mathrm{CO} \mathrm{CO} 2 \mathrm{CH} 4 \mathrm{H} 2 \mathrm{O}$ Frac $=0.0 \quad 0.0 \quad 0.0$ \& 0.00 .95

; Flash-Specs Agas2 Temp $=425 \quad P=200$ Maxit $=300$ Tol $=0.001 /$ Wwater Temp $=425 \quad P=200$ Maxit $=300$ Tol $=0.001$

Biock Hwcool Heater 


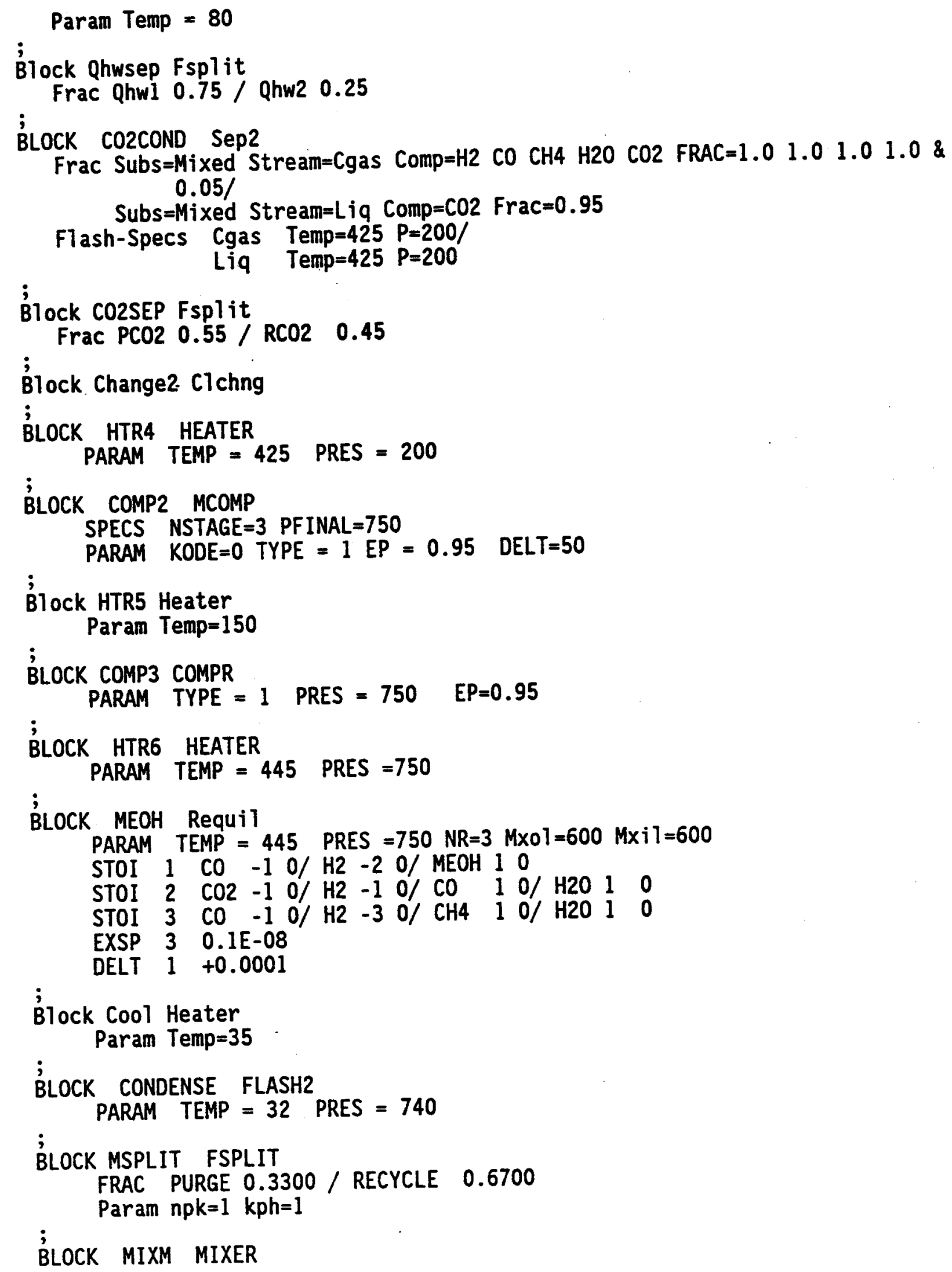




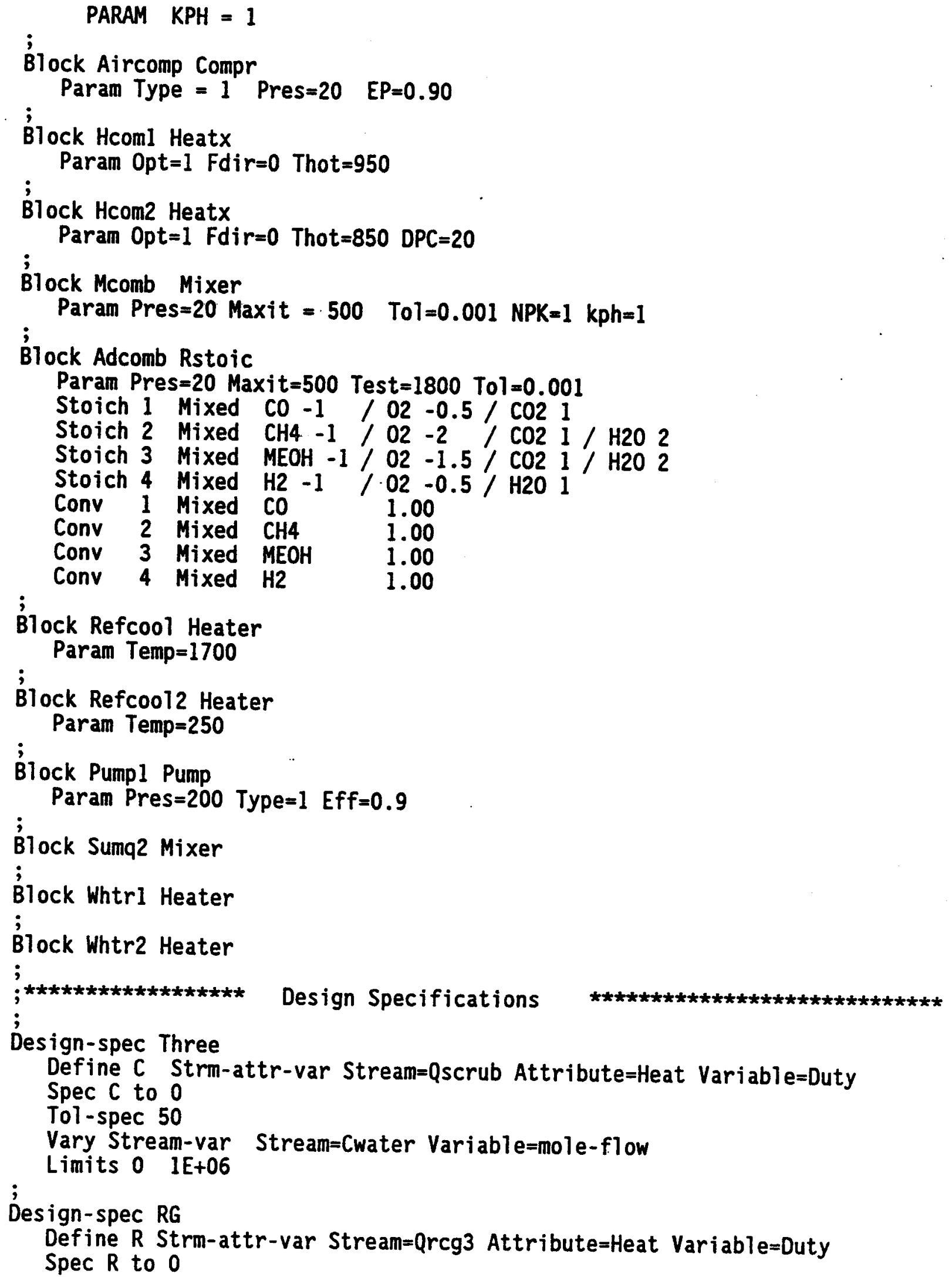


Tol-spec 100

Vary Stream-var Stream=Rgwat Variable=Mole-flow

Limits 0 0.1e+07

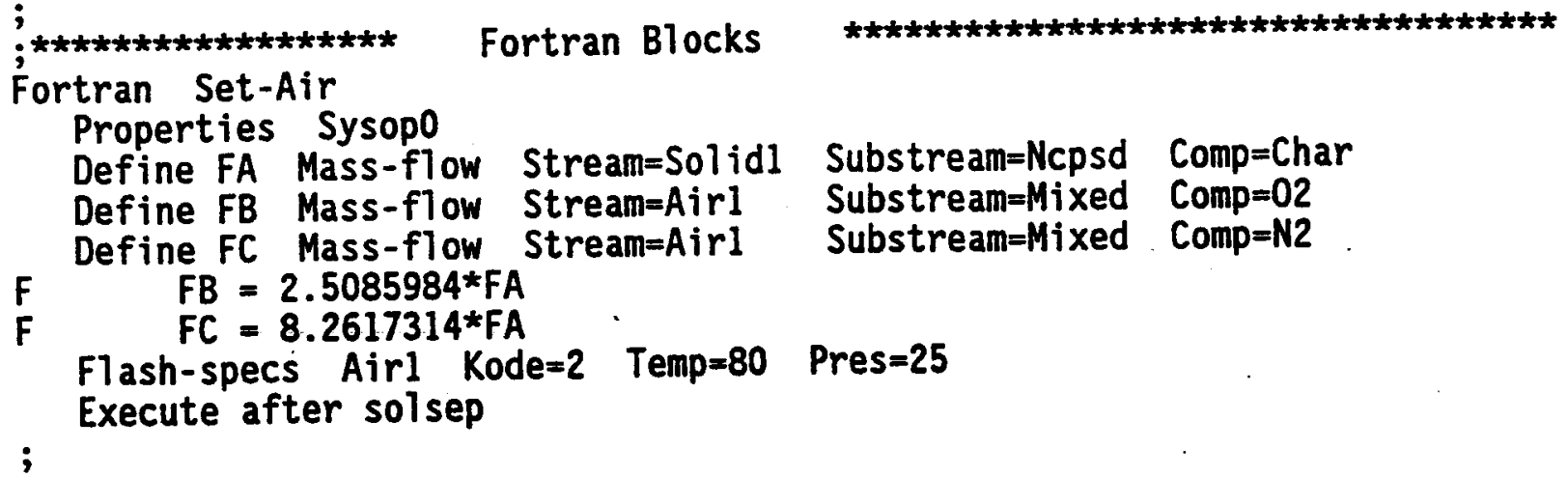




\section{REPORT DOCUMENTATION PAGE}

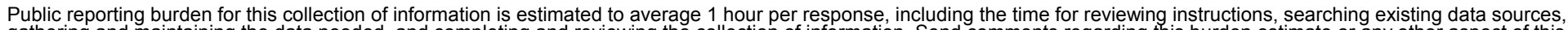

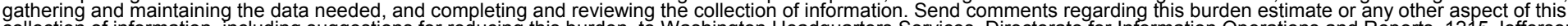

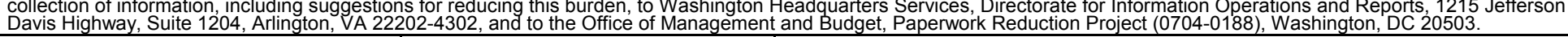

\begin{tabular}{|l|l|l|}
\hline 1. AGENCY USE ONLY (Leave blank) & $\begin{array}{c}\text { 2. REPORT DATE } \\
\text { January } 1992\end{array}$ & $\begin{array}{l}\text { 3. REPORT TYPE AND DATES COVERED } \\
\text { Technical Report }\end{array}$ \\
\hline
\end{tabular}

4. TITLE AND SUBTITLE
Material and Energy Balances for Methanol from Biomass Using Biomass Gasifiers

BF15.3436

6. AUTHOR(S)

R.L. Bain

7. PERFORMING ORGANIZATION NAME(S) AND ADDRESS(ES)

National Renewable Energy Laboratory

1617 Cole Blvd.

Golden, CO 80401-3393

9. SPONSORING/MONITORING AGENCY NAME(S) AND ADDRESS(ES)

8. PERFORMING ORGANIZATION

REPORT NUMBER

NREL/TP-510-17098

10. SPONSORING/MONITORING AGENCY REPORT NUMBER

11. SUPPLEMENTARY NOTES

12a. DISTRIBUTION/AVAILABILITY STATEMENT

National Technical Information Service

12b. DISTRIBUTION CODE

U.S. Department of Commerce

5285 Port Royal Road

Springfield, VA 22161

13. ABSTRACT (Maximum 200 words)

The objective of the Biomass to Methanol Systems Analysis Project is the determination of the most economically optimum combination of unit operations which will make the production of methanol from biomass competitive with or more economic than traditional processes with conventional fossil fuel feedstocks. This report summarizes the development of simulation models for methanol production based upon the Institute of Gas Technology (IGT) "Renugas" gasifier and the Battelle Columbus Laboratory (BCL) gasifier. This report discusses methanol production technology, the IGT and BCL gasifiers, analysis of gasifier data for gasification of wood, methanol production material and energy balance simulations, and one case study based upon each of the gasifiers.

14. SUBJECT TERMS

Biomass to methanol; simulation models; methanol; biomass; biomass gasifiers

15. NUMBER OF PAGES

16. PRICE CODE

17. SECURITY CLASSIFICATION OF REPORT Unclassified

NSN 7540-01-280-5500
19. SECURITY CLASSIFICATION OF ABSTRACT Unclassified
20. LIMITATION OF ABSTRACT

UL

SECURITY CLASSIFICATION
OF THIS PAGE
Unclassified

